\title{
A new look at some aspects of geometry, particle physics, inertia, radiation and cosmology
}

\author{
G.Ter-Kazarian*1 \\ ${ }^{1}$ Byurakan Astrophysical Observatory, 378433, Aragatsotn District, Armenia
}

\begin{abstract}
Continuing along the line of our previous report (Ter-Kazarian, 2021c), in present communication we briefly outline several closely related issues, carried out also in Byurakan Astrophysical Observatory, not touched in it for brevity reasons. These issues reveal and further develop novel aspects of the fundamental nature and structure of the space-time geometry and the high energy physics, the inertia effects, the intense radiation physics, and the notion of relative velocity in a curved space-time.
\end{abstract}

Keywords: geometry — torsion - supersymmetric quantum mechanics-operator manifold - Standard Model of elementary particles - supersymmetric extension — inertia effects — radiation physics — cosmology

\section{Contents}

\section{Introduction} 2

2. Two-step spacetime deformation-induced dynamical torsion

3. The TSSD-metric-affine gravity behind the spacetime deformation

4. An extended phase space stochastic quantization of constrained hamiltonian systems

5. An extended phase space SUSY quantum mechanics

6. Classical analog of extended phase space SUSY and its breaking 23

7. Operator manifold approach to geometry and particle physics 26

8. OM: Field aspect 28

9. OM: Differential geometric aspect 30

10. Primordial structures and link establishing processes 31

11. Operator Multimanifold $\hat{G}_{N}$

12. Microscopic theory of the Standard Model of elementary particles 37

13. Supersymmetric Extension of MTSM 43

14. Spacetime deformation induced inertia effects 49

15. Probing inertia behind the SUSY 53

16. Einstein's transition coefficients for Compton scattering 64

17. The theory of Multiphoton Comptonization 66

18. Unique definition of relative velocity of luminous source as measured along
the observer's line-of-sight in a generic pseudo-Riemannian space-time

19. The implications for the spatially homogeneous and isotropic

Robertson-Walker space-time 72

20. The physical outlook and concluding remarks 74

21. References 78

*gago_50@yahoo.com, Corresponding author 


\section{Introduction}

In our previous report (Ter-Kazarian, 2021c), among others, we have also developed on the new physical perception of space-time geometry - the theory of distortion of space-time continuum (DSTC) (Ter-Kazarian, 1986, 1989c, 1997, 2010) at huge energies (respectively, at short distances $<0.4 \mathrm{fm})$. Using the language of fundamental geometric structure-distortion gauge induced fiberbundle, it leads to modified gravitational theory as a corollary of the spacetime deformation/distortion framework. We generalize the standard gauge scheme via the concept of distortion gauge field which acts on the external spacetime groups. This theory, in turn, underlies the microscopic theory of black hole (Ter-Kazarian, 1989a,b, 1990, 1991, 1992, 2001a, Ter-Kazarian \& Yerknapetian, 1995), which has smeared out the central singularities of BHs, and makes room for their growth and merging behavior. The MTBH is the first-principles treatment of a fundamental 'superdense protomatter' physics, but it also has an actual physical realization of Ambartsumian's fundamental vision, which enables an insight to key puzzles of ultra-high energy astrophysics (Ter-Kazarian, 2014, 2015, 2016a,b, Ter-Kazarian \& Sargsyan, 2013, Ter-Kazarian \& Shidhani, 2017, 2019, Ter-Kazarian et al., 2003, 2006, 2007).

Continuing along this line, in present communication we briefly outline several closely related issues, carried out also in Byurakan Astrophysical Observatory, not touched in previous paper for brevity reasons. These issues reveal novel aspects of the fundamental nature and structure of space-time geometry and high energy physics, inertia effects and intense radiation physics. In the same time, the interested reader is invited to consult for details the original papers as follows: Two-step spacetime deformation (TSSD)-induced dynamical torsion (Ter-Kazarian, 2011); TSSD-metric-affine gravity behind the spacetime deformation (Ter-Kazarian, 2015); Extended phase space SUSY (Ter-Kazarian, 2009, 2013a, Ter-Kazarian \& Sobouti, 2008); The operator manifold approach to geometry and particle physics (TerKazarian, 1884, 1996, 1999a); Microscopic theory of the Standard Model (MTSM) of elementary particles (Ter-Kazarian, 1999b, 2001b); and Supersymmetric extension of MTSM (Ter-Kazarian, 2001c); Spacetime deformation induced inertia effects (Ter-Kazarian, 2010, 2012)); Probing inertia behind the SUSY (Ter-Kazarian, 2001b); Einstein's transition coefficients for Compton scattering, the annihilation and creation of electron-positron pairs at intense radiation (Ter-Kazarian, 1984c,e); The theory of Multiphoton Comptonization (Ter-Kazarian, 1984a,b,d, 1987, 1989a,b); Unique definition of relative velocity of luminous source as measured along the observer's line-of-sight in a generic pseudoRiemannian space-time (Ter-Kazarian, 2021b): The implications for the spatially homogeneous and isotropic Robertson-Walker space-time (Ter-Kazarian, 2021a).

With this perspective in sight, we will proceed according to the following structure. To start with, Section 2 recounts some of the highlights behind of the TSSD-induced dynamical torsion (Ter-Kazarian, 2011, 2015). We extend the geometrical ideas of the spacetime deformations (Ter-Kazarian, 2010, 2012) to study the physical foundation of post-Riemannian geometry. To this aim, we construct the theory of TSSD as a guiding principle. Through a non-trivial choice of explicit form of a deformation tensor, we have a way to derive different post Riemannian spacetime structures such as: (i) the Weitzenböck spacetime structure - $\left(W_{4}\right)$ underlying a teleparallelism theory of gravity; (ii) the RC manifold $\left(U_{4}\right)$ underlying Einstein-Cartan theory also called Einstein-Cartan-Sciama-Kibble theory; (iii) or even the most general linear connection of metric-affine gravity (MAG) theory taking values in the Lie-algebra of the $4 \mathrm{D}$-affine group, $A(4, R)=R^{4} \otimes G L(4, R)$ (the semi-direct product of the group of $4 \mathrm{D}$-translations and general linear $4 \mathrm{D}$-transformations). We address the theory of teleparallel gravity and construct a consistent Einstein-Cartan (EC) theory with the dynamical torsion. We show that the equations of the standard EC theory, in which the equation defining torsion is the algebraic type and, in fact, no propagation of torsion is allowed, can be equivalently replaced by the set of modified Einstein-Cartan equations in which the torsion, in general, is a dynamical. The special physical ansatz for the spacetime deformations yields the short-range propagating torsion.

Having gained some insight into the physical foundation of the Einstein-Cartan theory, with twostep spacetime deformation induced dynamical torsion, TSSD- $U_{4}$ theory, in Section 3, we extend these ideas as applied to the more general TSSD-metric-affine gravity (Ter-Kazarian, 2015). The MAG theory is an extension of the Poincaré gauge theory of gravity, constructed in the Rieman-Cartan geometry, to the most general spacetime symmetry gauge theory. The MAG theory has the most general type of covariant derivative: in addition to curvature and torsion, the MAG also has nonmetricity, i.e., 
a nonmetric compatible connection. Hence parallel transport no longer preserves length and angle. The TSSD-MAG is constructed in the framework of the first order Lagrangian expressed in terms of the gauge potentials and their first derivatives. We show that, in this framework, the equations of the standard MAG theory which have no further propagating modes can be equivalently replaced by the set of the modified equations which, in the limit of reducing the affine group, may recover TSSD- $U_{4}$ theory, with propagating torsion. The special physical ansatz for the spacetime deformations yields the short-range propagating torsion. In testing the modified TSSD-MAG framework for different particular cases, the restrictions are imposed via the method of Lagrange multipliers. To pursue the TSSD-approach further, here we address the essential features of the MAG theory in context of the TSSD-construction of post-Riemannian geometry. A formulation of the major physical aspects of this theory will be given in the framework of the first order Lagrangian expressed in terms of the gauge potentials and their first derivatives. All the fundamental gravitational structures in fact - the metric as much as the coframes and connections - acquire a TSSD induced theoretical interpretation. It is remarkable that in the framework of the first order Lagrangian, the equations of the standard MAG theory which have no further propagating modes can be equivalently replaced by the set of the modified equations which, in the limit of reducing the affine group, may recover TSSD- $U_{4}$ theory, with a dynamical torsion.

In Section 4, we give an outline of the 'extended phase space' stochastic quantization of constrained hamiltonian systems (Ter-Kazarian \& Sobouti, 2008). Exploring the concept of 'actual and virtual paths' in a phase space formalism, we address a stochastic quantization of Hamiltonian systems with first class holonomic constraints. Extended canonical transformations allows to go from one extended phase space to another. This unifying feature of the theory makes the comparison of the various functions existing in the literature possible and transparent. We have developed the stochastic quantization method (SQM) in extended phase space and shown how this method can be generalized to deal with systems subjected to first class constraints.

All this variety prompts us, in Section 5, to address the SUSY for an 'extended phase space' quantum mechanical system (Ter-Kazarian, 2009). We have concerned ourselves with the extended phase space quantum mechanics of particles which have both bosonic and fermionic degrees of freedom, i.e., the quantum field theory in $(0+1)$-dimensions in $q-$ (position) and $p-$ (momentum) spaces, exhibiting supersymmetry. We present $(\mathrm{N}=2)$-realization of the supersymmetry algebra, and discuss the vacuum energy and the topology of super-potentials. We demonstrate the merits of shape-invariance of exactly solvable extended SUSY potentials, which has underlying algebraic structure, by obtaining analytic expressions for the entire energy spectrum of extended Hamiltonian with Scarf potential without ever referring to underlying differential equation. However, a shape-invariance is not the most general integrability condition as not all exactly solvable potentials seem to be shape-invariant. As an application we obtain analytic expressions for the entire energy spectrum of extended Hamiltonian with Scarf potential without ever referring to underlying differential equation.

In Section 6, we derive the classical analog of the `extended phase space' SUSY quantum mechanics and obtain the integrals of motion (Ter-Kazarian, 2013a). Consequently, we describe the extended phase space ( $\mathrm{N}=2$ )-SUSY algebra. In the second part, by means of an iterative scheme, first, we find the approximate groundstate solutions to the extended Schrödinger-like equation, and then calculate the parameters which measure the breaking of extended SUSY such as the groundstate energy. We calculate a more practical measure for the SUSY breaking, in particular in field theories which is the expectation value of an auxiliary field. We analyze non-perturbative mechanism for extended phase space SUSY breaking in the instanton picture and show that this has resulted from tunneling between the classical vacua of the theory. Finally, we deal with the independent group theoretical methods with nonlinear extensions of Lie algebras from the perspective of extended phase space SUSY quantum mechanics and, further, shows how it can be useful for spectrum generating algebra.

Section 7 offers a detailed analysis of the operator manifold approach to geometry and particle physics (Ter-Kazarian, 1884, 1996, 1999a). The fundamental question that guides our discussion is how did the geometry and particles come into being? To explore this query, the operator manifold (OM)-formalism enables the unification of the geometry and the field theory. It yields the quantization of geometry drastically different from earlier suggested schemes. It address itself to the question of the 
A new look at some aspects of geometry, particle physics, inertia, radiation and cosmology

prime-cause of origin of geometry and basic concepts of particle physics such as the fundamental fields of quarks and leptons with the spins and various quantum numbers, internal symmetries and so forth; also basic four principles of Relativity, Quantum, Gauge and Color Confinement, which are, as it was proven, all derivative and come into being simultaneously. The substance out of which the geometry and particles are made is a set of new physical structures. The most promising aspect of our approach so far is the fact that many of the important anticipated properties, basic concepts and principles of particle physics are appeared quite naturally in the framework of suggested theory. In pursuing the original problem further we have elaborated a new mathematical framework, which is, in fact, a still wider generalization of familiar methods of secondary quantization with appropriate expansion over the geometric objects. The OM formalism provides a natural unification of the geometry- yielding the special and general relativity principles, and the quantized fermion fields serving as the basis for the constituent subquarks.

In Section 8, we discuss the key points of field aspect of OM. The quantum field theory of the $\mathrm{OM}$ is equivalent to configuration space wave mechanics employing the antisymmetric state functions incorporated with geometric properties of corresponding objects. Therein, by applying the algebraic approach we reach to rigorous definition of the OM. Considering an arbitrary superposition of state vectors we get a whole set of explicit forms of the matrix elements of operator vector and covector fields.

In Section 9, we briefly outline the key points of differential geometric aspect of OM. The operators are the basis for all operator vectors of tangent section of principle bundle. The smooth field of tangent operator vector is a class of equivalence of the curves. For any function of the ordinary class of functions of smoothness, one may define an operator differential. Constructing matrix elements of operator tensors, one produces the Cartan's exterior forms. Whence, the matrix elements of symmetric operator tensors equal zero. The differential operator form at given point can be defined as the exterior operator form on tangent operator space of tangent operator vectors. The linear operator form of 1 degree is a linear operator valued function. The set of all linear operator forms defined at given point fill up the operator vector space. We consider the integration of operator form. Next, we apply the analog of exterior differentiation.

Section 10 deals with primordial structures and link establishing processes. It has cleared up the physical conditions in which the geometry and particles come into being.

These structures are thought to be the substance out of which the geometry and particles are made. There is not any restriction on the number of primordial structures of both types involved in the link establishing processes simultaneously. Only, in the stable system the link stability condition must be held for each linkage separately. The persistent processes of creation and annihilation of the primordial structures proceed in different states. The processes of creation and annihilation of 'regular structures' in lowest state are described by the OM formalism given above. In all higher states the primordial structures are distorted (interaction states) and described by distorted link functions defined on distorted manifolds. The 'distorted ordinary structures' emerge in geometry only in permissible combinations forming a stable system. Below, in simplified schematic way we exploit the background of the known colour confinement and gauge principles. Naive version of such construction still should be considered as a preliminary one, which will be further elaborated to introduce a basis for the subquarks. Due to the incompatibility commutation relations the transformation matrices generate the unitary groups of internal symmetries $U(1), S U(2), S U(3)$ corresponding to one-, twoand three-dimensional rotations, while an action of physical system must be invariant under such transformations (gauge principle). The subquarks emerge in the geometry only in certain permissible combinations utilizing the idea of the subcolour (subquark) confinement principle, and have undergone the transformations yielding the internal symmetries and gauge principle.

In Section 11, we generalize the OM-formalism via the concept of operator multimanifold (OMM), which yields the multiworld (MW) geometry involving the spacetime continuum and internal worlds of given number. All this is not merely an exercise in abstract reasoning but presumably bears directly on the geometry of the universe in which we live. In an enlarged framework of the OMM we define and clarify the conceptual basis of subquarks and their characteristics stemming from the various symmetries of the internal worlds. The hypothesis of existence of the MW structures manifests 
its virtue by solving some key problems of particle phenomenology, when we attempt to suggest a microscopic approach to the properties of particles and interactions.

A definite pattern for the theoretical description of particle physics has emerged based on the framework of the Standard Model (SM) of high energy physics, which is built up from observation for prediction and correlation of the new data. Although the SM has proven to be in spectacular agreement with experimental measurements and quite successful in a predicting a wide range of phenomena, however, it is not exception to the rule that as the phenomenological approach it suffers from own difficulties. There were still many open key questions arisen inevitably that we have no understanding why the SM is as it is? Why is the gauge symmetry? Why is this the particle spectrum? The mechanism of the electroweak symmetry breaking is a complete mystery. A phenomenological standard model (SM) of high energy physics with enormous success settles order in entangled experimental data. Although it has proven to be in spectacular agreement with experimental measurements and highly successful in a description and predicting a wide range of phenomena, however, it suffers from some vexing problems and many key questions of both the phenomenological and SUSY aspects have yet to be answered. To fill the void which the SM presents, and to innovate the solution to alluded problems, we will use OM-formalism as a backdrop to discuss in Section 12 the key points of Microscopic theory of the Standard Model (MTSM) of elementary particles (Ter-Kazarian, 1999b, 2001b). A theoretical significance of the MSM, first of all, resides in the microscopic interpretation of all physical parameters.

With this perspective in sight, in Section 13, we further expose the assertions made in OMformalism, to recount some of the highlights behind of the supersymmetrization of MTSM (SuMTSM) (Ter-Kazarian, 2001c). We promote the MTSM into supersymmetric framework in order to solve its technical aspects of vacuum zero point energy and hierarchy problems, and attempt, further, to develop its realistic viable minimal SUSY extension. Among other things that - the MTSM provides a natural unification of geometry and the field theory, has clarified the physical conditions in which the geometry and particles come into being, in microscopic sense enables an insight to key problems of particle phenomenology and answers to some of its nagging questions - a present approach also leads to quite a new realization of the SUSY yielding a physically realistic particle spectrum. It stems from the special subquark algebra, from which the nilpotent supercharge operators are derived. The resulting theory makes plausible testable implications for the current experiments at LEP2, at the Tevatron and at LHC drastically different from those of the conventional MSSM models.

In Section 14, we further discuss the Spacetime deformation induced inertia effects (Ter-Kazarian, 2010, 2012). We construct a toy model of spacetime deformation induced inertia effects, in which we prescribe to each and every particle individually a new fundamental constituent of hypothetical 2D, so-called, master-space (MS), subject to certain rules. The MS, embedded in the background $4 \mathrm{D}$-spacetime, is an indispensable companion to the particle of interest, without relation to every other particle. The MS is not measurable directly, but we argue that a deformation/(distortion of local internal properties) of MS is the origin of inertia effects that can be observed by us. With this perspective in sight, we construct the alternative relativistic theory of inertia (RTI) (Ter-Kazarian, 2010, 2012). We go beyond the hypothesis of locality with special emphasis on distortion of MS, which allows to improve essentially the standard metric and other relevant geometrical structures referred to a noninertial frame in Minkowski spacetime for an arbitrary velocities and characteristic acceleration lengths. We compute the inertial force exerted on the photon in a gravitating system in the semiRiemann space. Despite the totally different and independent physical sources of gravitation and inertia, this approach furnishes justification for the introduction of the weak principle of equivalence (WPE), i.e., the universality of free fall. Consequently, we relate the inertia effects to the more general post-Riemannian geometry. We derive a general expression of the relativistic inertial force exerted on the extended spinning body moving in the Rieman-Cartan space.

In Section 15, we prob the inertia behind SUSY. We derive a standard Lorentz code (SLC) of motion by exploring rigid double transformations of, so-called, master space-induced supersymmetry (MS-SUSY), subject to certain rules (Ter-Kazarian, 2001b). The renormalizable and actually finite flat-space field theories with $N_{\max }=4$ supersymmetries in four dimensions, if only such symmetries are fundamental to nature, yield the possible extension of Lorentz code (ELC), at which the SLC violating new physics appears. In the framework of local MS-SUSY, we address the inertial effects. 
We argue that a space-time deformation of MS is the origin of inertia effects that can be observed by us. We go beyond the hypothesis of locality. This allows to improve the relevant geometrical structures referred to the noninertial frame in Minkowski space for an arbitrary velocities and characteristic acceleration lengths. This framework furnishes justification for the introduction of the weak principle of equivalence, i.e., the universality of free fall. The implications of the inertia effects in the more general post-Riemannian geometry are briefly discussed.

In Section 16, we briefly outline the issues on the interaction of electrons with the intense radiation: Einstein's transition coefficients for Compton scattering, and the annihilation and creation of electron-positron pairs at intense radiation (Ter-Kazarian, 1984c,e). Einstein's ideas are developed for free-virtual, virtual-free and free-free transitions for electron-photon scattering at arbitrary intense radiation by splitting Compton scattering into two components. Whereas, we consider the general problem of interaction of electrons with the intense radiation via $s$-photon Compton scattering $s \gamma+e \rightarrow \gamma^{\prime}+e^{\prime}$. In doing this, we introduce a new concept of 'effective photon', and then instead of $s$-photon scattering by electron with an 'effective' four-momentum, with equal footing,, we should consider the scattering of one 'effective photon' by free electron. The Compton scattering is the $s$-channel of the photon-electron interaction. This formalism can be easily extended to the $t$-channel of the photon-electron interaction, namely to processes of annihilation and creation of electron-positron pairs. Certainly, since the Feynman diagram for these processes is topologically identical to the corresponding diagram of the $s$-channel of the photon-electron interaction, then the probability coefficients for the $t$-channel of the photon-electron interaction can be obtained directly from the Compton scattering by performing simple replacements. Determining the probability coefficients of transitions in the state of thermodynamic equilibrium, we readily derive the radiation transfer equation for nonequilibrium processes.

On this basis, in Section 17, we suggest the theory of multiphoton comptonization (Ter-Kazarian, 1984a,b,d, 1987, 1989a,b). Employing the method of 'effective photons', the integral kinetic equation is derived that describes the time variation of the distribution function of quanta of non-equilibrium intense radiation for their multiphoton Compton scattering on Maxwellian nonrelativistic electrons. This equation is the crux for the constructed theory of multiphoton comptonization, which determines the heating of thermal electrons during multiphoton induced Compton interaction. The integral kinetic equation allows one to describe the evolution of intense spectral lines of radiation, for any spectral widths, and any angular aperture of the radiation beam.

In Section 18, we discuss the unique definition of relative velocity of luminous source as measured along the observer's line-of-sight in a generic pseudo-Riemannian space-time. Using a way of separating the spectral shifts into infinitesimally displaced 'relative' spectral bins and sum over them, in Subsection 18.1, we overcome the ambiguity of the parallel transport of four-velocity, in order to give an unique definition of the so-called kinetic relative velocity of luminous source as measured along the observer's line-of-sight in a generic pseudo-Riemannian space-time (Ter-Kazarian, 2021b). A resulting relationship between the spectral shift and the kinetic relative velocity is utterly distinct from a familiar global Doppler shift rule. We show that such a performance of having found a kinetic relative velocity of luminous source, without subjecting it to a parallel transport, manifests its virtue in particular case when adjacent observers are being in free fall and populated along the null geodesic. So that the kinetic relative velocity is reduced to global Doppler velocity as studied by Synge (Synge, 1960).

In Section 19, we discuss the implications for the spatially homogeneous and isotropic RobertsonWalker space-time (Ter-Kazarian, 2021a), which leads to cosmological consequences that the resulting kinetic recession velocity of a galaxy is always subluminal even for large redshifts of order one or more, and thus, it does not violate the fundamental physical principle of causality.

The physical outlook and concluding remarks are given in Section 20.

\section{Two-step spacetime deformation-induced dynamical torsion}

The TSSD as a guiding principle. When considering several connections with different curvature and torsion, one takes spacetime simply as a manifold, and connections as additional structures. The 
universality of gravitation allows the Levi-Civita connection to be interpreted as part of the spacetime definition. The form of Riemannian connection, which is a function of the tetrad fields and their derivatives, shows that the relative orientation of the orthonormal frame $\breve{e}_{a}(\breve{x}+d \breve{x})$ with respect to $\breve{e}_{a}(\breve{x})$ (parallel transported to $(\breve{x}+d \breve{x})$ is completely fixed by the metric. Since a change in this orientation is described by Lorentz transformations, it does not induce any gravitational effects; therefore, from the point of view of the Principle of Equivalence, there is no reason to prevent independent (due to arbitrary deformations) Lorentz rotations of local frames in the space under consideration. If we want to use this freedom, the spin connection should contain a part which is independent of the metric, which will realize an independent Lorentz rotation of frames under parallel transport. In this way, we are led to a description of gravity which is not in Riemann space, but in the metric-affine geometry. If all inertial frames at a given point are treated on an equal footing, the spacetime has to have torsion, which is the antisymmetric part of the affine connection. Recall that the concept of a linear connection as an independent and primary structure of spacetime is the fundamental proposal put forward by Élie Cartan's geometrical analysis. Cartan gave a beautiful geometrical interpretation of torsion and curvature. Namely, the torsion is related to the translation of a vector, like curvature is related to the rotation of a vector, when it is displaced around an infinitesimal closed path - loop, and when this loop is developed in the flat space tangent to the manifold such that the tangent space rolls without gliding around the loop. At the end of the journey one has a rotation if there is only curvature, or the loop, mapped into the tangent space, has a small closure failure, i.e. a translational misfit, a translation if there is only torsion, or both if there is curvature and torsion. When torsion is nonvanishing, the affine connection is no longer coincident with the Levi-Civita connection, and the geometry is no longer Riemannian, but one has a Riemann-Cartan $U_{4}$ spacetime, with a nonsymmetric, but metric-compatible, connection. On the other hand, teleparallel gravity attributes gravitation to torsion, but in this case torsion accounts for gravitation not by geometrizing the interaction, but by acting as a force. This theory represented a new way of including torsion into general relativity, an alternative to the scheme provided by the usual Einstein-Cartan-Sciama-Kibble approach. However, for a specific choice of the free parameters, teleparallel gravity shows up as a theory completely equivalent to Einstein's general relativity, in which case it is usually referred to as the teleparallel equivalent of general relativity. From this point of view, curvature and torsion are simply alternative ways of describing the gravitational field, and consequently related to the same degrees of freedom of gravity. The fundamental difference between these two theories above was that, whereas in the former torsion is a propagating field, in the latter it is not, a point which can be considered a drawback of this model. This prompt us below to separate these two different cases.

\subsection{Model building: spacetime deformations}

When considering several connections with different curvature and torsion, one takes spacetime simply as a manifold, and connections as additional structures. From this view point, below we shall tackle the problem of spacetime deformation. To start with, let us consider the holonomic metric defined in the Riemann space, $V_{4}$, as $\breve{g}=\breve{g}_{\mu \nu} \breve{\vartheta}^{\mu} \otimes \breve{\vartheta}^{\nu}=\breve{g}\left(\breve{e}_{\mu}, \breve{e}_{\nu}\right) \breve{\vartheta}^{\mu} \otimes \breve{\vartheta}^{\nu}$, with components, $\breve{g}_{\mu \nu}=\breve{g}\left(\breve{e}_{\mu}, \breve{e}_{\nu}\right)$ in dual holonomic base $\left\{\breve{\vartheta}^{\mu} \equiv d \breve{x}^{\mu}\right\}$. All magnitudes related with the Riemann space, $V_{4}$, will be denoted with an over ' ${ }^{\prime}$. The space, $V_{4}$, has at each point a tangent space, $\breve{T}_{\breve{x}} V_{4}$, spanned by the four tetrad fields, $\breve{e}_{a}=\breve{e}_{a}{ }^{\mu} \breve{\partial}_{\mu}$, which relate $\breve{g}$ to the tangent space metric, $o_{a b}=\operatorname{diag}(+---)$, by $o_{a b}=\breve{g}\left(\breve{e}_{a}, \breve{e}_{b}\right)=\breve{g}_{\mu \nu} \breve{e}_{a}{ }^{\mu} \breve{e}_{b}{ }^{\nu}$. The coframe members are $\breve{\vartheta}^{b}=\breve{e}_{\mu}^{b} d \breve{x}^{\mu}$, such that $\left.\breve{e}_{a}\right\rfloor \breve{\vartheta}^{b}=\delta_{a}^{b}$, where \rfloor denotes the interior product, namely, this is a $C^{\infty}$-bilinear map $\rfloor: \Omega^{1} \rightarrow \Omega^{0}$ with $\Omega^{p}$ denotes the $C^{\infty}$-modulo of differential p-forms on $V_{4}$. In components $\breve{e}_{a}{ }^{\mu} \breve{e}_{\mu}^{b}=\delta_{a}^{b}$. One can consider general transformations of the linear group, $G L(4, R)$, taking any base into any other set of four linearly independent fields. The notation, $\left\{\breve{e}_{a}, \breve{\vartheta}^{b}\right\}$, will be used below for general linear frames. The converse metric is $\breve{g}_{\mu \nu}=o_{a b} \breve{e}^{a}{ }_{\mu} \breve{e}^{b}{ }_{\nu}$ because of $\breve{e}_{a}{ }^{\mu} \breve{e}^{a}{ }_{\nu}=\delta_{\nu}^{\mu}$. The anholonomy objects read $\breve{C}^{a}:=d \breve{\vartheta}^{a}=$ $\frac{1}{2} \breve{C}_{b c}^{a} \breve{\vartheta}^{b} \wedge \breve{\vartheta}^{c}$, where the anholonomy coefficients, $\breve{C}_{b c}^{a}$, which represent the curls of the base members are $\breve{C}^{c}{ }_{a b}=-\breve{\vartheta}^{c}\left(\left[\breve{e}_{a}, \breve{e}_{b}\right]\right)=\breve{e}_{a}{ }^{\mu} \breve{e}_{b}{ }^{\nu}\left(\breve{\partial}_{\mu} \breve{e}^{c}{ }_{\nu}-\breve{\partial}_{\nu} \breve{e}^{c}{ }_{\mu}\right)=-\breve{e}_{\mu}{ }_{\mu}\left[\breve{e}_{a}\left(\breve{e}_{b}{ }^{\mu}\right)-\breve{e}_{b}\left(\breve{e}_{a}{ }^{\mu}\right)\right]$. The (anholonomic) Levi-Civita (or Christoffel) connection can be written as $\left.\left.\left.\breve{\Gamma}_{a b}:=\breve{e}_{[a}\right\rfloor d \breve{\vartheta}_{b]}-\frac{1}{2}\left(\breve{e}_{a}\right\rfloor \breve{e}_{b}\right\rfloor d \breve{\vartheta}_{c}\right) \wedge \breve{\vartheta}^{c}$ where $\breve{\vartheta}_{c}$ is understood as the down indexed one-form $\breve{\vartheta}_{c}=o_{c b} \breve{\vartheta}^{b}$. Next, we write a norm, $d s$, of 
the infinitesimal displacement, $d x^{\mu}$, on the general smooth differential $4 \mathrm{D}$-manifold $\mathcal{M}_{4}$, in terms of the spacetime structures of $V_{4}$, as $d s=\Omega_{\mu}{ }^{\nu} \breve{e}_{\nu} \breve{\vartheta}^{\mu}=\Omega_{b}{ }^{a} \breve{e}_{a} \breve{\vartheta}^{b}=e_{\rho} \vartheta^{\rho}=e_{a} \vartheta^{a} \in \mathcal{M}_{4}$, where $\Omega_{\mu}{ }^{\nu}$ is the world-deformation tensor, $\left\{e_{a}=e_{a}{ }^{\rho} e_{\rho}\right\}$ is the frame and $\left\{\vartheta^{a}=e^{a}{ }_{\rho} \vartheta^{\rho}\right\}$ is the coframe defined on $\mathcal{M}_{4}$, such that $\left.e_{a}\right\rfloor \vartheta^{b}=\delta_{a}^{b}$, or in components, $e_{a}{ }^{\mu} e^{b}{ }_{\mu}=\delta_{a}^{b}$, also the procedure can be inverted $e^{a}{ }_{\rho} e_{a}{ }^{\sigma}=\delta_{\rho}^{\sigma}$. Provided, $\Omega_{\mu}{ }^{\nu}=\pi_{\mu}{ }^{\rho} \pi_{\rho}{ }^{\nu}, \Omega_{b}{ }^{a}=\pi_{c}{ }^{a} \pi_{b}{ }^{c}=\Omega_{\mu}{ }^{\nu} \breve{e}^{a}{ }_{\nu} \breve{e}_{b}{ }^{\mu}, e_{\rho}=\pi_{\rho}{ }^{\nu} \breve{e}_{\nu} \equiv \partial_{\rho}$, $\vartheta^{\rho}=\pi_{\mu}^{\rho} \breve{\vartheta}^{\mu} \equiv d x^{\rho}, \quad x^{\rho} \in \mathcal{U} \in \mathcal{M}_{4}$. Hence the deformation tensor, $\Omega^{a}{ }_{b}$, yields a local tetrad deformations $e_{a} \vartheta^{a}=\Omega_{{ }_{b}}^{a} \breve{e}_{a} \breve{\vartheta}^{b}, \quad e_{c}=\pi_{c}{ }^{a} \breve{e}_{a}, \quad \vartheta^{c}=\pi_{b}^{c} \breve{\vartheta}^{b}$. A general spin connection then transforms according $\omega_{b \mu}^{a}=\pi_{c}{ }^{a} \breve{\omega}_{d \mu}^{c} \pi_{b}^{d}+\pi_{c}{ }^{a} \partial_{\mu} \pi_{b}^{c}$. The matrices, $\pi(\bar{x}):=\left(\pi_{b}{ }^{a}\right)(\bar{x})$, can be called first deformation matrices, and the matrices $\gamma_{c d}(x)=o_{a b} \pi_{c}{ }^{a}(x) \pi_{d}{ }^{b}(x)$, as the second deformation matrices. The matrices, $\pi_{c}^{a}(x) \in G L(4, R) \forall x$, in general, give rise to right cosets of the Lorentz group, i.e. they are the elements of the quotient group $G L(4, R) / S O(3,1)$.

\subsection{The post-Riemannian geometry}

In what follows, we deal with a more generic spacetime deformation $\pi(x)$, consisted of a double deformations $(\dot{\pi}(\dot{x}), \sigma(x))$ of the infinitesimal displacements described on a generic smooth differential 4D manifold in terms of the spacetime structures of a Riemann space. The first deformation matrix $\dot{\pi}(\dot{x})$, will be conveniently chosen in such a way that the deformed connection is set as the Weitzenböck connection, and the associated deformed spin-connection vanishes. the Weitzenböck connection is a connection presenting a non-vanishing torsion, but vanishing curvature. This recovers a particular case of the teleparallel gravity theory with the dynamical torsion. All magnitudes related with the teleparallel gravity will be denoted with an over ' $\bullet$ '. Then, we will be able, further, to generalize the Einstein-Cartan equations for which the spin generates a dynamical torsion part, associated with spacetime deformation $\sigma(x)$, in the canonical energy-momentum tensor producing a deviation from Riemannian geometry. Keeping in mind aforesaid, let a deformation $(\breve{e}, \breve{\vartheta}) \rightarrow(e, \vartheta)$ be now performed in the two-steps:

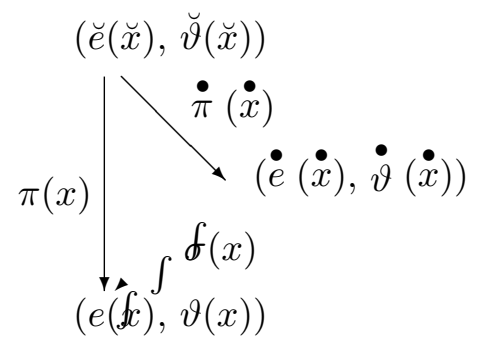

Two-step deformation map

where the first deformation matrix, $\dot{\pi}(\dot{x}):=\left(\dot{\pi}_{b}{ }_{b}{ }^{a}\right)(\dot{x})$, is the solution of the following equation: $\dot{\pi}_{c}{ }_{c}^{a}(\dot{x}) \dot{\partial}_{\mu} \dot{\pi}^{-1}{ }_{b}{ }_{b}(\dot{x})=\breve{\omega}_{b \mu}^{a}(\breve{x})$, where $\breve{\omega}_{b \mu}^{a}(\breve{x})$ is the spin connection defined in the Riemann space. We recall that for an arbitrary matrix M, $\operatorname{Tr}\left\{M^{-1} \partial_{\mu} M\right\}=\partial_{\mu} \ln \operatorname{Det} M$, where Det- denotes the determinant, $\operatorname{Tr}$ - the trace. Then, in matrix notation $\dot{\pi}:=\left(\dot{\pi}_{b}{ }^{a}\right)$ and $\breve{\omega}_{\mu}:=\left(\breve{\omega}_{b \mu}^{a}\right)$, we have $\operatorname{Tr}\left\{\dot{\pi}(\dot{x}) \dot{\partial}_{\mu} \dot{\pi}^{-1}(\dot{x})\right\}=-\dot{\partial}_{\mu} \ln \operatorname{Det} \dot{\pi}(\dot{x})=\operatorname{Tr} \breve{\omega}_{\mu}(\breve{x})$, which gives Det $\dot{\pi}(\dot{x})=\operatorname{Det} \dot{\pi}_{0}$ $\exp \left\{-\int_{0}^{\dot{x}} \operatorname{Tr} \breve{\omega}_{\mu}(\breve{x}) d \dot{x}^{\prime \mu}\right\}$. Hence $\dot{\pi}(\dot{x})=\dot{\pi}(0) \exp \left[-\int_{0}^{\dot{x}} \breve{\omega}_{\mu}(\breve{x}) d \dot{x}^{\prime \mu}\right]$, where $\dot{\pi}(0) \equiv C \dot{\pi}_{0}, C$ is an arbitrary proper constant matrix $|C|=1$. Provided, $\dot{\Omega}_{\mu}{ }^{\nu}=\dot{\pi}_{\mu}{ }_{\mu} \dot{\pi}_{\rho}{ }_{\rho}{ }, \dot{\Omega}_{b}{ }^{a}=\dot{\pi}_{c}{ }_{c}{ }_{\pi}{ }^{c}{ }_{b}=\dot{\Omega}_{\mu}{ }_{\mu} \breve{e}^{a}{ }_{\nu} \breve{e}_{b}{ }^{\mu}$, $\dot{e}_{\rho}=\dot{\pi}_{\rho}{ }_{\rho}^{\nu} \breve{e}_{\nu} \equiv \dot{\partial}_{\rho}=\frac{\partial}{\partial \dot{x}^{\rho}}, \dot{\vartheta}^{\rho}=\dot{\pi}^{\rho}{ }_{\mu} \breve{\vartheta}^{\mu} \equiv d \dot{x}^{\rho}$. Under a local spacetime deformation $\dot{\pi}(\dot{x})$, the tetrad changes according to $\dot{e}_{a} \dot{\vartheta}^{a}=\dot{\Omega}_{b}^{a} \breve{e}_{a} \breve{\vartheta}^{b}, \dot{e}_{c}=\dot{\pi}_{c}{ }_{c} \breve{e}_{a}, \dot{\vartheta}^{c}=\dot{\pi}_{b}^{c} \breve{\vartheta}^{b}$, and, the general deformed spin connection vanishes $\dot{\omega}_{b \mu}^{a}=\dot{\pi}_{c}{ }_{c}^{a} \breve{\omega}^{c}{ }_{d \mu} \dot{\pi}_{b}{ }_{b}+\dot{\pi}_{c}{ }_{c}^{a} \dot{\partial}_{\mu} \dot{\pi}_{b}{ }_{b}=\dot{e}^{a}{ }_{\sigma} \dot{\Gamma}_{\rho \mu}^{\sigma}{ }_{\rho} \dot{e}_{b}{ }^{\rho}+\dot{e}^{a}{ }_{\rho} \dot{\partial}_{\mu} \dot{e}_{b}{ }^{\rho} \equiv 0$. In fact, a general linear connection, $\dot{\Gamma}_{\rho \sigma}^{\mu}$, is related to the corresponding spin connection, $\dot{\omega}_{b \mu}^{a}$, through the inverse $\dot{\Gamma}_{\rho \sigma}^{\mu}=\dot{e}{ }_{a}^{\mu} \dot{\partial}_{\sigma} \dot{e}_{\rho}^{a}+\dot{e}_{a}{ }^{\mu} \dot{\omega}_{b \sigma}^{a}{ }_{e}{ }_{\rho}^{b}=\dot{e}_{a}{ }^{\mu} \dot{\partial}_{\sigma} \dot{e}_{\rho}^{a}$, which is the the Weitzenböck connection revealing the Weitzenböck spacetime $W_{4}$ of the teleparallel gravity (see next subsect.). Thus, 
$\dot{\dot{\pi}}(\dot{x})$ can be referred to as the Weitzenböck deformation matrix. The above equations are simply different ways of expressing the property that the total - that is, acting on both indices - derivative of the tetrad vanishes identically. According to the two-step spacetime deformations map, the next first deformation matrices $\sigma(x):=\left(\sigma_{b}{ }^{a}\right)(x)$, contribute to corresponding ingredient part, $\chi_{b}{ }^{d}$, of general deformation tensor, $\Omega_{b}{ }^{a}=\chi_{b}{ }^{d} \dot{\Omega}_{d}{ }^{a}=\chi_{b}{ }^{d} \dot{\widetilde{\Omega}}_{\rho}{ }_{\rho} \breve{e}_{\nu}{ }^{a} \breve{e}_{d}{ }_{d}, \bar{\chi}_{d}{ }^{c}=\sigma_{e}{ }^{c} \sigma_{d}{ }^{e}, \bar{\chi}_{e}{ }^{d} \dot{\pi}_{b}{ }^{e}=\chi_{b}{ }^{e} \dot{\pi}_{e}{ }^{d}$, or $\Omega_{\mu}{ }^{\nu}=\chi_{\mu}{ }^{\rho} \dot{\widetilde{\Omega}}{ }_{\rho}{ }^{\nu}, \chi_{\mu}{ }^{\rho}=\chi_{b}{ }^{d} \breve{e}_{d}{ }^{\rho} \breve{e}^{b}{ }_{\mu}$. Under a deformation, $\sigma(x)$, in general, the tetrad changes according to $e_{c}=\left(\sigma_{c}{ }^{d} \dot{\pi}_{d}^{a}\right) \breve{e}_{a}=\sigma_{c}{ }^{d} \dot{e}_{d}, \vartheta^{c}=\left(\sigma_{e}^{c} \dot{\pi}_{b}^{e}\right) \breve{\vartheta}^{b}=\sigma_{e}^{c} \dot{\vartheta}^{e}, e_{\rho}=\sigma_{\rho}{ }^{\sigma} \dot{e}_{\sigma}, \vartheta^{\rho}=\sigma_{\sigma}{ }^{\rho} \dot{\vartheta}^{\sigma}$, $e_{\rho}=\sigma_{\rho}^{c} \dot{e}_{c}, \vartheta^{\rho}=\sigma_{c}{ }^{\rho} \dot{\vartheta}^{c}, \sigma_{\rho}^{c}=\sigma_{\rho}{ }^{\sigma} \dot{e}_{\sigma^{c}}{ }^{c}, \sigma^{\rho}{ }_{c}=\sigma_{\sigma}^{\rho} \dot{e}^{\sigma}{ }_{c}, e_{c} \vartheta^{c}=\bar{\chi}_{d}{ }^{c} \dot{e}_{c} \dot{\vartheta}^{d}=\Omega_{b}{ }^{a} \breve{e}_{a} \breve{\vartheta}^{b}$. The corresponding second deformation matrices read $\gamma_{c d}(x)=\bar{\chi}_{e e^{\prime}} \dot{\pi}_{c}^{e} \dot{\pi}_{d}^{e^{\prime}}, \dot{\gamma}_{c d}(\dot{x})=o_{a b} \dot{\pi}_{c}^{a}(\dot{x}) \dot{\pi}_{d}^{b}(\dot{x})$, where $\bar{\chi}_{e e^{\prime}}=o_{a b} \sigma_{e}^{a} \sigma_{e^{\prime}}^{b}$. Under a local tetrad deformation, a general spin connection transforms according to $\omega_{b \mu}^{\prime a}=\sigma_{c}{ }^{a} \dot{\omega}^{c}{ }_{d \mu} \sigma_{b}^{d}+\sigma_{c}{ }^{a} \partial_{\mu} \sigma_{b}^{c}$, such that $\stackrel{(\sigma)}{\omega}_{a \mu}^{a}:=\omega_{b \mu}{ }_{b \mu}=\sigma_{c}{ }^{a} \partial_{\mu} \sigma_{b}^{c}$, is referred to as the deformation related frame connection, which represents the deformed properties of the frame only. Then, it follows that the affine connection, $\Gamma$, related to tetrad deformations, transforms through $\Gamma_{\rho \sigma}^{\mu}=e_{a}{ }^{\mu} \partial_{\sigma} e_{\rho}^{a}+e_{a}{ }^{\mu} \stackrel{(\pi)}{\omega}_{a}{ }_{b \sigma} e_{\rho}^{b}=\sigma_{a}{ }^{\mu} \partial_{\sigma} \sigma_{\rho}^{a}+\sigma_{a}{ }^{\mu} \stackrel{(\sigma)}{\omega}_{a}{ }_{b \sigma} \sigma_{\rho}^{b}$, where $\sigma_{a}{ }^{\mu} \sigma_{\mu}{ }^{b}=\delta_{a}^{b}$, also the procedure can be inverted $\sigma_{a}{ }^{\mu}{\sigma_{\nu}}^{a}=\delta_{\nu}^{\mu}$, and that $\stackrel{(\pi)}{\omega}_{b \mu}$ : $:=\omega_{b \mu}^{a}=\pi_{c}^{a} \breve{\omega}^{c}{ }_{d \mu} \pi^{d b}+\pi_{c}^{a} \partial_{\mu} \pi^{c b}$, is the spin connection. Then, the line element, $d s^{2}$ may alternatively be recast in general form of the spacetime or frame objects, respectively, as $d s^{2}=g_{\mu \nu} \vartheta^{\mu} \otimes \vartheta^{\nu}=g\left(e_{\mu}, e_{\nu}\right) \vartheta^{\mu} \otimes \vartheta^{\nu}=\left(\Omega_{\mu}{ }^{\nu} \Omega_{\rho}{ }^{\sigma}\right) \breve{g}_{\nu \sigma} \breve{\vartheta}^{\mu} \otimes \breve{\vartheta}^{\rho}=o_{a b} \vartheta^{a} \otimes \vartheta^{b}=$ $\left(\Omega_{a}^{c} \Omega_{b}{ }^{d}\right) o_{c d} \breve{\vartheta}^{a} \otimes \breve{\vartheta}^{b}=\gamma_{c d} \breve{\vartheta}^{c} \otimes \breve{\vartheta}^{d}$. For our convenience, the notation, $\left\{\begin{array}{c}(A) \\ e\end{array} \underset{a}{(A)},{ }^{(A)} b\right\}(A=\pi, \sigma)$, will be used below for general linear frames $\{\stackrel{(A)}{e} \underset{a}{a}, \stackrel{(A)}{\vartheta} b\}=\left\{\left(\stackrel{(\pi)}{e}_{a}, \stackrel{(\sigma)}{e}_{a}\right),\left(\stackrel{(\pi)}{\vartheta}{ }^{b}, \stackrel{(\sigma)}{\vartheta}^{b}\right)\right\} \equiv\left\{\left(e_{a}, \dot{e}_{a}\right),\left(\vartheta^{b}, \dot{\vartheta}^{b}\right)\right\}$, where $\left.\stackrel{(A)}{e}{ }_{a}\right\rfloor \stackrel{(A)}{\vartheta} b=\delta_{a}^{b}$, or in components, $\stackrel{(A)}{e}{ }_{a}^{\mu} \stackrel{(A)}{e}_{\mu}{ }_{\mu}=\delta_{a}^{b}$, also the procedure can be inverted $\stackrel{(A)}{e} \underset{\rho}{a} \stackrel{(A)}{e} \underset{a}{\sigma}=\delta_{\rho}^{\sigma}$. Provided, $\stackrel{(A)}{e} a_{a}^{\mu}=\left(\stackrel{(\pi)}{e} a_{a}^{\mu}, \stackrel{(\sigma)}{e} a_{a}^{\mu}\right) \equiv\left(e_{a}{ }^{\mu}, \sigma_{a}{ }^{\mu}\right)$. Hence, the affine connection can be re-written in the abbreviated form $\Gamma_{\rho \sigma}^{\mu}=\stackrel{(A)}{e} a^{\mu} \partial_{\sigma} \stackrel{(A)}{e} \underset{\rho}{a}+\stackrel{(A)}{e}_{a}^{\mu} \stackrel{(A)}{\omega}_{b \sigma} \stackrel{(A)}{e} b_{\rho}$. Since the first deformation matrices $\pi(x)$ and $\sigma(x)$ are arbitrary functions, the transformed general spin connections $\stackrel{(\pi)}{\omega}(x)$ and $\stackrel{(\sigma)}{\omega}(x)$, as well as the affine connection, are independent of tetrad fields and their derivatives. In what follows, therefore, we will separate the notions of space and connectionsthe metric-affine formulation of gravity. A metric-affine space $\left(\mathcal{M}_{4}, g, \Gamma\right)$ is defined to have a metric and a linear connection that need not dependent on each other. The new geometrical property of the spacetime, are the nonmetricity one-form $N_{a b}$ and the affine torsion two-form $T^{a}$ representing a translational misfit. These, together with the curvature two-form $R_{a}^{b}$, symbolically can be presented as $\left(N_{a b}, T^{a}, R_{a}^{b}\right) \sim \mathcal{D}\left(g_{a b}, \vartheta^{a}, \Gamma_{a}^{b}\right)$, where $\mathcal{D}$ is the covariant exterior derivative. We may introduce the contortion tensors related to the deformation related frame connection and the spin connection: $\stackrel{(A)}{K} c_{a \nu}=\stackrel{(A)}{\omega} c_{a \nu}+\stackrel{(A)}{\Delta} c_{a \nu}$, , where $\stackrel{(A)}{\Delta}_{\mu \rho \nu}=\stackrel{(A)}{e}_{\mu a} \stackrel{(A)}{e}_{[\rho, \nu]}^{a}-\stackrel{(A)}{e}_{\rho a} \stackrel{(A)}{e}_{[\mu, \nu]}^{a}-\stackrel{(A)}{e}_{\nu a} \stackrel{(A)}{e}_{[a, \rho]}^{a}$, is referred to as the the Ricci coefficients of rotation. Both the contortion tensor and spin connection are antisymmetric in their first two indices. The relations between the corresponding torsion and contortion tensors $\operatorname{read} \stackrel{(A)}{K}_{\rho}^{\rho}{ }_{\mu \nu}: \quad=2 \stackrel{(A)}{Q} \underset{(\mu \nu)}{\rho}+\stackrel{(A)}{Q} \rho_{\mu \nu}^{\rho}, \stackrel{(A)}{Q}^{\rho}{ }_{\mu \nu}=\stackrel{(A)}{K}^{\rho}{ }_{[\mu \nu]}$, where $\stackrel{(A)}{Q} \rho_{\mu \nu}^{\rho}=\stackrel{(A)}{\omega} \rho_{[\mu \nu]}+\stackrel{(A)}{e} a_{[\mu, \nu]} \stackrel{(A)}{e} \underset{a}{\rho}$. Let us define then a translation in the connection space. Suppose a point in this space will be a Lorentz connection, $\stackrel{(\pi)}{\omega}(x):=\stackrel{(\pi)}{\omega}^{b c}{ }_{\mu}(x) J_{b c} d x^{\mu}$, presenting simultaneously curvature and torsion written in the language of differential forms as $\stackrel{(\pi)}{R}=d \stackrel{(\pi)}{\omega}+\stackrel{(\pi)}{\omega} \underset{(\pi)}{\omega} \equiv \mathcal{D}_{(\underset{\omega}{\omega}} \stackrel{(\pi)}{\omega}, \quad \stackrel{(\pi)}{T}=d e+\stackrel{(\pi)}{\omega} e \equiv \mathcal{D}_{(\pi)} e$, where $\mathcal{D}_{(\underset{\omega}{(\pi)})}$ denotes the covariant differential in the connection $\stackrel{(\pi)}{\omega}$. For more detail see (Ter-Kazarian, 2011).

\subsection{Teleparallel gravity}

The total covariant derivative of a geometrical object carrying both flat and curvilinear indices is covariant with respect to both diffeomorphism and local Lorentz symmetries. In both, $W_{4}$ and 
$U_{4}$, spaces the total covariant derivative of the vierbein field, $e^{a}{ }_{\nu}$, is assumed to vanish $\mathcal{D}_{\mu} e^{a}{ }_{\nu}=$ $\partial_{\mu} e^{a}{ }_{\nu}-\Gamma_{\nu \mu}^{\rho} e_{\rho}^{a}+\omega_{b \mu}^{a} e^{b}{ }_{\nu}=0$, which provides a relation between both connections. We may now introduce the Weitzenböck torsion $\dot{T}^{\rho}{ }_{\mu \nu}: \quad=\dot{\Gamma}^{\rho}{ }_{\mu \nu}-\dot{\Gamma}^{\rho}{ }_{\nu \mu}$, and the Weitzenböck contortion: $\dot{K}$ ${ }_{\mu \nu}: \quad=\frac{1}{2}\left(\dot{T}_{\nu}{ }_{\mu}{ }_{\mu}+\dot{T}_{\mu}{ }^{\rho}{ }_{\nu}+\dot{T}^{\rho}{ }_{\mu \nu}\right)$. We then obtain $\dot{T}^{\rho}{ }_{\mu \nu}=T_{\mu \nu}^{\rho}-\omega^{\rho}{ }_{\mu \nu}+\omega^{\rho}{ }_{\nu \mu}$. and $\dot{K}^{\rho}{ }_{\mu \nu}=$ $K^{\rho}{ }_{\mu \nu}+\omega^{\rho}{ }_{\mu \nu}$. Below, we will concentrate on the specific space, $W_{4}$, of vanishing affine torsion in the class of frames, $\left\{\dot{e}_{a}\right\}: T_{\mu \nu}^{\rho}=0$. While, the metricity condition holds: $\dot{N}_{a b}:=-\dot{\mathcal{D}} g_{a b}=0$, and that $\Gamma_{\mu \nu}^{\rho}=\stackrel{\circ}{\Gamma}_{\mu \nu}^{\rho}$, as in the Riemann space. Consequently, $K_{\mu \nu}^{\rho}=0$, and $\dot{K}^{\rho}{ }_{\mu \nu}=\omega^{\rho}{ }_{\mu \nu}$. Hence, $\dot{\circ}^{\rho}{ }_{\mu \nu}=\stackrel{\circ}{\Gamma}^{\rho}{ }_{\mu \nu}+\stackrel{\circ}{K}^{\rho}{ }_{\mu \nu}=\Gamma^{\rho}{ }_{\mu \nu}+\dot{\circ}^{\rho}{ }^{\rho}{ }_{\mu \nu}$, while, the Weitzenböck covariant derivative of the tetrad field vanishes identically: $\dot{\mathcal{D}}_{\nu} \dot{e}^{a}{ }_{\mu} \equiv \dot{\partial}_{\nu} \dot{e}^{a}{ }_{\mu}-\dot{\varphi}^{\rho}{ }_{\mu \nu} \dot{e}^{a}{ }_{\rho}=0$. This is the so called distant, or absolute parallelism condition. As a consequence of this condition, the corresponding Weitzenböck spin connection also vanishes identically: $\dot{\omega}^{c}{ }_{a \nu}=\stackrel{\circ}{\omega}^{c}{ }_{a \nu}+\dot{K}^{c}{ }_{a \nu} \equiv 0$. Of course, these relations above are true only in one specific class of frames. In fact, since $\dot{\omega}^{c}{ }_{a \nu}$ is the Weitzenböck spin connection, if it vanishes in a given frame, it will be different from zero in any other frame related to the first by a local Lorentz transformation. In teleparallel gravity, the coupling of spinor fields with gravitation is a highly controversial subject. However, it seems there is no compelling arguments supporting the choice of the Weitzenböck spin connection $\dot{\omega}^{c}{ }_{a \nu}$ as the spin connection of teleparallel gravity, otherwise several problems are immediately encountered with such coupling prescription. The teleparallel gravity becomes consistent and fully equivalent with GR, even in the presence of spinor fields if we write the minimal coupling prescription as $\dot{\partial}_{a} \rightarrow \dot{\mathcal{D}}_{a}=\dot{e}_{a}{ }^{\mu} \dot{\mathcal{D}}_{\mu}$ with $\dot{\mathcal{D}}_{\mu}$ the teleparallel Fock-Ivanenko derivative written in the form $\dot{\mathcal{D}}_{\mu}$ : $\quad=\dot{\partial}_{\mu}-\frac{i}{2} \dot{\Omega}_{b \mu}{ }_{b \mu} J_{a}{ }^{b}$, where the teleparallel spin connection, $\dot{\Omega}_{b \mu}{ }_{b \mu}$, reads $\dot{\Omega}_{b \mu}^{a}:=0-\dot{K}_{b \mu}^{a}$. Field equations can be derived from the least action, $\delta \dot{S}=0$, with the total invariant action of conventional theory of teleparallel gravity.

\subsection{The TSSD-induced dynamical torsion in tensorial form}

In this section we construct the TSSD- $U_{4}$ theory, which considers curvature and torsion as representing independent degrees of freedom. The RC manifold, $U_{4}$, is a particular case of general metricaffine manifold $\mathcal{M}_{4}$, restricted by the metricity condition $N_{\lambda \mu \nu}=0$, when a nonsymmetric linear connection is said to be metric compatible. Taking the antisymmetrized derivative of the metric condition gives an identity between the curvature of the spin-connection and the curvature of the Christoffel connection $\stackrel{(A)}{R}_{\mu \nu}^{a b}(\stackrel{(A)}{\omega}) \stackrel{(A)}{e}_{\rho b}-R_{\rho \mu \nu}^{\sigma}(\Gamma) \stackrel{(A)}{e}_{\sigma}^{a}=0$, where $\stackrel{(A)}{R}_{\mu \nu}^{a b}(\stackrel{(A)}{\omega})=\partial_{\mu} \stackrel{(A)}{\omega}_{\nu}^{a b}-\partial_{\nu} \stackrel{(A)}{\omega}_{\mu}^{a b}+\stackrel{(A)}{\omega}_{\mu}^{a c} \stackrel{(A)}{\omega}^{(A)}$ $\nu_{\nu c}^{b}-\stackrel{(A)}{\omega}_{\nu}^{a c} \stackrel{(A)}{\omega}_{\mu c}^{b}, R_{\rho \mu \nu}^{\sigma}(\Gamma)=\partial_{\mu} \Gamma_{\nu \rho}^{\sigma}-\partial_{\nu} \Gamma_{\mu \rho}^{\sigma}-\Gamma_{\mu \rho}^{\lambda} \Gamma_{\nu \lambda}^{\sigma}+\Gamma_{\nu \rho}^{\lambda} \Gamma_{\mu \lambda}^{\sigma}$. Hence, the relations between the scalar curvatures for an $U_{4}$ manifold read $\stackrel{(A)}{R}(\stackrel{(A)}{\omega}) \equiv e^{(A)}{ }_{a}^{\mu} \stackrel{(A)}{e}_{b}{ }_{b} \stackrel{(A)}{R}_{\mu \nu}^{a b}(\stackrel{(A)}{\omega})=R(g, \Gamma) \equiv g^{\rho \nu} R_{\rho \mu \nu}^{\mu}(\Gamma)$. This means that the Lorentz and diffeomorphism invariant scalar curvature, $R$, becomes either a function of $\stackrel{(A)}{e} \underset{\mu}{a}$ only, or a function of $g_{\mu \nu}$ only. Certainly, it can be seen by noting that the Lorentz gauge transformations can be used to fix the six antisymmetric components of $\stackrel{(A)}{e}{ }_{\mu}^{a}$ to vanish. Then in both cases diffeomorphism invariance fixes four more components out of the six $g_{\mu \nu}$, with the four components $g_{0 \mu}$ being non dynamical, obviously, leaving only two dynamical degrees of freedom. This shows the equivalence of the vierbein and metric formulations holds.

The total Einstein-Cartan action can be written in the terms of the spin connection, $\stackrel{(\pi)}{\omega}$ and the deformation related frame connection, $\stackrel{(\sigma)}{\omega}$, in the form $\left.S=S_{g}^{(A)} \stackrel{(A)}{\omega}\right)+S_{m}^{(\pi)}(\stackrel{(\pi)}{\omega})=-\frac{1}{2 æ} \int \stackrel{(A)}{R}$ $\sqrt{-g} d \Omega+\int L_{m}^{(\pi)}(g, \Psi, \nabla \Psi) \sqrt{-g} d \Omega$, where $S_{g}^{(A)} \quad(A=\pi, \sigma)$ is the action for the gravitational field written in terms of scalar curvature $\stackrel{(A)}{R} \stackrel{(A)}{\omega})$ for an $U_{4}$ manifold, while $S_{m}^{(\pi)}$ is the action for the matter fields, $æ$ is the coupling constant relating to Newton gravitational constant $æ=8 \pi G / c^{4}$. The action regards the contortion tensor as a variational variable, in addition to the gravitational and matter fields. The gravitational action can be decomposed as $S_{g}^{(A)}=-\frac{1}{2 æ} \int \stackrel{\circ}{R} \sqrt{-g} d \Omega+S_{Q}^{(A)}$, where the 
torsional action reads $S_{Q}^{(A)}=\frac{1}{2 x} \int d \Omega \sqrt{-g} L_{Q}^{(A)}$ The coupling constant of the spin-tortion is the same of that of the mass-metric distortion field interaction. The dynamical spin density tensor, which is antisymmetric in the Lorentz indices, reads $\stackrel{(\pi)}{s}_{\mu}^{a b}=2 \frac{\delta\left(\sqrt{-g} L_{m}^{(\pi)}\right)}{\delta \omega_{a b}^{(\pi)}{ }_{a b}}=2 \frac{\delta\left(\sqrt{-g} L_{m}^{(\pi)}\right)}{\delta K_{a b}^{(\pi)}}=\sqrt{-g} \stackrel{(\pi)}{S}_{\mu}^{a b}$. The variation of the action have to be applied by independent variation of the fields $g, \stackrel{(\pi)}{\omega}$ (x) (or equivalently $\stackrel{(\pi)}{K}(x))$ and $\Psi(x), \bar{\Psi}(x)$. In terms of the Euler-Lagrange variations, the least action $\delta S=0$ gives $\delta g^{\mu \nu}: \quad \stackrel{\circ}{G}_{\mu \nu}+\frac{\delta\left(\sqrt{-g} L_{Q}^{(A)}\right)}{\delta g_{\mu \nu}}=-2 æ \frac{\delta\left(\sqrt{-g} L_{m}^{(\pi)}\right)}{\delta g^{\mu \nu}} ; \delta \stackrel{(\pi)}{\omega}_{\nu}^{\mu \rho}: \frac{\partial \stackrel{(A)}{\omega} \mu_{\nu^{\prime}}^{\mu^{\prime} \rho^{\prime}}}{\partial \stackrel{(\pi)}{\omega}_{\nu}^{\mu \rho}} \frac{\delta}{\delta{ }_{\nu}^{(A)} \mu_{\nu^{\prime}}^{\mu^{\prime}}}\left(\sqrt{-g} L_{Q}^{(A)}\right)=$ $-\frac{\delta\left(\sqrt{-g} L_{m}^{(\pi)}\right)}{\delta \stackrel{(\pi)}{\omega}_{\nu}^{\mu \rho}} ; \delta \Psi: \quad \frac{\delta\left(\sqrt{-g} L_{m}^{(\pi)}\right)}{\delta \Psi}=0 ; \quad \delta \bar{\Psi}: \quad \frac{\delta\left(\sqrt{-g} L_{m}^{(\pi)}\right)}{\delta \bar{\Psi}}=0$, where $\stackrel{\circ}{G}_{\mu \nu}$ is the Einstein tensor. Hence, the first Einstein-Cartan equation is written $\stackrel{\circ}{G}_{\mu \nu}=æ \stackrel{(A)}{\theta}_{\mu \nu}$, where including the spin contributions directly into the energy-momentum tensor, we introduce the canonical energy-momentum tensor $\stackrel{(A)}{\theta}{ }_{\mu \nu}$ : $=T_{\mu \nu}+\stackrel{(A)}{U}_{\mu \nu}$. For variations $\delta \stackrel{(\pi)}{K}^{\nu}{ }_{\mu \rho}$ (or equivalently $\stackrel{(\pi)}{\omega}^{\nu}{ }_{\mu \rho}$ ), the $\delta S=0$ gives the second

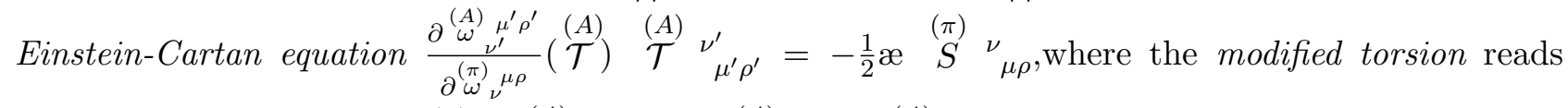
$\stackrel{(A)}{\mathcal{T}}_{\mu \rho}{ }_{\mu \rho}: \quad=\frac{1}{2 \sqrt{-g}} \frac{\delta\left(\sqrt{-g} L_{Q}^{(A)}\right)}{\delta \stackrel{(A)}{\omega}_{\nu} \mu \rho}=\stackrel{(A)}{Q} \nu_{\mu \rho}+\delta_{\mu}^{\nu} \stackrel{(A)}{Q}_{\rho}-\delta_{\rho}^{\nu} \stackrel{(A)}{Q}_{\mu}$. Thus, the equations of the standard Einstein-Cartan theory can be recovered for $A=\pi: \stackrel{\circ}{G}_{\mu \nu}=æ \stackrel{(\pi)}{\theta}_{\mu \nu}, \quad \stackrel{(\pi)}{\mathcal{T}}{ }_{\mu \rho}=-\frac{1}{2} æ \stackrel{(\pi)}{S}^{\nu}{ }_{\mu \rho}$, in which the equation defining torsion is the algebraic type, such that torsion at a given point in spacetime does not vanish only if there is matter at this point, represented in the Lagrangian density by a function which depends on torsion. Unlike the metric, which is related to matter through a differential field equation, torsion does not propagate. However, these equations can be equivalently replaced by the set of modified Einstein-Cartan equations for $A=\sigma: \stackrel{\circ}{G}_{\mu \nu}=æ \stackrel{(\sigma)}{\theta}_{\mu \nu}, \quad \Theta_{\nu^{\prime} \mu \rho}^{\mu^{\prime} \rho^{\prime} \nu}(\stackrel{(\sigma)}{\mathcal{T}}) \stackrel{(\sigma)}{\mathcal{T}} \nu_{\mu^{\prime} \rho^{\prime}}=-\frac{1}{2} æ \stackrel{(\pi)}{S}^{\nu}{ }_{\mu \rho}$,

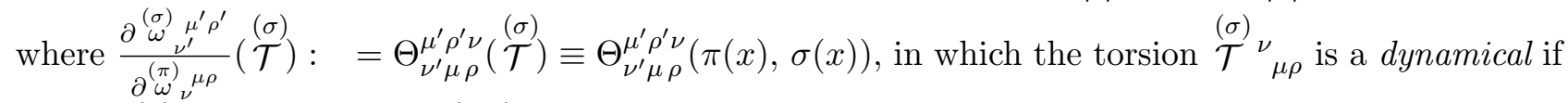
only $\Theta_{\nu^{\prime} \mu \rho}^{\mu^{\prime} \rho^{\prime} \nu}(\pi(x), \sigma(x)) \neq \delta_{\mu}^{\mu^{\prime}} \delta_{\rho}^{\rho^{\prime}} \delta_{\nu^{\prime}}^{\nu}$. Therefore, it is spin that generates a nonsymmetric part in the canonical energy-momentum tensor and then, produces a deviation from the Riemann geometry. The variation of $S_{m}^{(\pi)}$ with respect to the metric-compatible affine connection in the metric-affine variational formulation of gravity is equivalent to the variation with respect to the torsion (or contortion) tensor. Consequently, the dynamical spin density $\stackrel{(\pi)}{S}_{a b}^{\mu}$ is identical with $\stackrel{(\pi)}{\Sigma}_{a b}^{\mu}=\frac{\partial\left(\sqrt{-g} L_{m}\right)^{(\pi)}}{\partial \Psi_{, \mu}} \stackrel{(\pi)}{S}_{a b} \Psi$, referred to as the canonical spin density. The canonical tensor $e \stackrel{(A)}{\theta}_{\mu \nu}=\tau_{\mu \nu}=e_{a \nu} \tau_{\mu}^{a}$ is generally not symmetric, whereas the canonical energy-momentum density is identical with the dynamical tetrad energy-momentum density $e \stackrel{(A)}{\theta} \underset{\mu}{a}=\tau_{\mu}^{a}$, where $e: \quad=\operatorname{det}\left|e_{a}^{\mu}\right|=\sqrt{-g}$. The relation between the tetrad dynamical energy-momentum tensor and the metric dynamical energy-momentum tensor for matter fields is $\stackrel{(A)}{\theta}{ }_{(\mu \nu)}=T_{\mu \nu}$. The Belinfante-Rosenfeld relation, between the dynamical metric and dynamical tetrad (canonical) energy-momentum tensors, can be written as $\stackrel{(A)}{\theta} \mu \nu-T_{\mu \nu}=\frac{1}{2} \nabla_{\nu}^{*}(\stackrel{(\pi)}{S}$ $\left.\mu \rho_{\mu}^{\nu}-\stackrel{(\pi)}{S}_{\rho}{ }_{\rho}^{\nu}+\stackrel{(\pi)}{S}^{\nu}{ }_{\mu \rho}\right)=\stackrel{(A)}{U} \mu \nu$, where $\nabla_{\mu}^{*}=\nabla_{\mu}-2 \stackrel{(\pi)}{Q}_{\mu}$ is the modified covariant derivative. The conservation law for the spin density results from antisymmetrizing the Belinfante-Rosenfeld relation with respect to the indices $\mu, \rho: \stackrel{(\pi)}{s}_{\mu \nu ; \rho}^{\rho}=\tau_{\mu \nu}-\tau_{\nu \mu}+2 \stackrel{(\pi)}{Q}_{\rho} \stackrel{(\pi)}{S}_{\mu \nu}^{\rho}$ or $\frac{1}{2} \nabla_{\rho}^{*} \stackrel{(\pi)}{S} \underset{\mu \nu}{\rho}=\stackrel{(A)}{\theta}[\mu \nu]$.

\subsection{TSSD- $U_{4}$ theory in the language of the differential forms}

In this subsection we re-derive the field equations of the TSSD- $U_{4}$ theory by using the exterior calculus. The fields have to be expressed in terms of differential forms in order to build the total 
Lagrangian four-form as the appropriate integrand of the action. Let $\stackrel{(A)}{\omega}^{a b}=\stackrel{(A)}{\omega}{ }_{\mu}^{a b} \wedge d x^{\mu}$ be the one-forms of corresponding connections assuming values in the Lorentz Lie algebra. The action for gravitational field can be written in the form $S_{g}=\stackrel{\circ}{S}+S_{Q}=-\frac{1}{4 æ} \int \star \stackrel{\circ}{R}+S_{Q}$, provided, $\stackrel{\circ}{R} \equiv \stackrel{\circ}{\stackrel{R}{R}}$ cd $\wedge \dot{\vartheta}^{c} \wedge \dot{\vartheta}^{d}=\stackrel{\circ}{R}(\pi)$ cd $\wedge \vartheta^{c} \wedge \vartheta^{d}$. Consider a phenomenological action of the spin-torsion interaction, $S_{Q}$, such that the variation of the connection one-form $\stackrel{(A)}{\omega}$ ab yields $\delta S_{Q}=\frac{1}{\nsim} \int \star \stackrel{(A)}{\mathcal{T}}_{a b} \wedge \delta \stackrel{(A)}{\omega} a b$, where $\star \stackrel{(A)}{\mathcal{T}}_{a b}:=\frac{1}{2} \star\left(\stackrel{(A)}{Q}_{a} \wedge \stackrel{(}{e}_{b}\right)$, and that $\stackrel{(A)}{Q} a=\stackrel{(A)}{D} \stackrel{(A)}{\vartheta} a=d \stackrel{(A)}{\vartheta} a_{+}+\stackrel{(A)}{\omega} a_{b} \wedge \stackrel{(A)}{\vartheta} b$, with the

wedge product monomial, $\stackrel{(A)}{\vartheta} \mu \nu \alpha$, defined on the $U_{4}$ space. The variation of the action describing the macroscopic matter sources $S_{m}^{(\pi)}$ with respect to coframe $\vartheta^{a}$, and connection one-form $\stackrel{(\pi)}{\omega}$ ab reads $\delta S_{m}=\int \delta L_{m}=\int\left(-\star \stackrel{(A)}{\theta} a \wedge \delta \stackrel{(A)}{\vartheta} a+\frac{1}{2} \star \stackrel{(\pi)}{\Sigma}_{a b} \wedge \delta \stackrel{(\pi)}{\omega} a b\right)$, where $\star \stackrel{(A)}{\theta}_{a}$ is the dual three-form relating to the canonical energy-momentum tensor, $\stackrel{(A)}{\theta} \underset{a}{\mu}$, by $\star \stackrel{(A)}{\theta}{ }_{a}=\frac{1}{3 !} \stackrel{(A)}{\theta} \underset{a}{\mu} \varepsilon_{\mu \nu \alpha \beta} \stackrel{(A)}{\vartheta} \nu \alpha \beta$. and $\star \stackrel{(\pi)}{\Sigma}_{a b}=-\star \stackrel{(\pi)}{\Sigma}_{b a}$ is the dual three-form corresponding to the canonical spin tensor, which is identical with the dynamical spin tensor $\stackrel{(\pi)}{S}_{a b c}$, namely $\star \stackrel{(\pi)}{\Sigma}_{a b}=\stackrel{(\pi)}{S}_{\mu}^{\mu}{ }_{a b} \varepsilon_{\mu \nu \alpha \beta} \vartheta^{\nu \alpha \beta}$. The integral $\stackrel{\circ}{S}=-\frac{1}{4 æ} \int \star \stackrel{\circ}{R}=$ $-\frac{1}{4 æ} \int \star \stackrel{\circ}{R}_{c d} \wedge \stackrel{(A)}{\vartheta}^{c} \wedge \stackrel{(A)}{\vartheta}^{d}$, is the usual Einstein action, written in the language of the exterior forms. Actually, writing explicitly the holonomic indices, we have $\stackrel{\circ}{S}=-\frac{1}{8 æ} \int \stackrel{\circ(A)}{R} \underset{\mu \nu}{a b} \stackrel{(A)}{e}{ }_{\alpha}^{c} \stackrel{(A)}{e}$ ${ }_{\beta}^{d} \varepsilon_{a b c d} \stackrel{(A)}{\vartheta}^{\mu \nu \alpha \beta}=-\frac{1}{8 æ} \int \stackrel{\circ}{\circ} \stackrel{(A)}{R}_{\mu \nu}^{a b} \varepsilon_{a b \alpha \beta} \varepsilon^{\mu \nu \alpha \beta} d \Omega$. Also, one may readily verify that $\stackrel{\circ}{S}=-\frac{1}{2 æ} \int \stackrel{\circ}{R}$ ${ }_{\mu \nu}^{a b} \stackrel{(A)}{e} \underset{\alpha}{[\mu} \stackrel{(A)}{e}_{\beta}^{\nu]} e d \Omega=-\frac{1}{2 æ} \int \stackrel{\circ}{R} \sqrt{-g} d \Omega$. and $\delta S_{Q}=\frac{1}{x} \int \stackrel{(A)}{\mathcal{T}}_{\mu \nu}^{\beta} \delta \stackrel{(A)}{\omega}{ }_{\beta}^{\mu \nu}$. The variation of the total action, given by the sum of the gravitational field action and the matter action, with respect

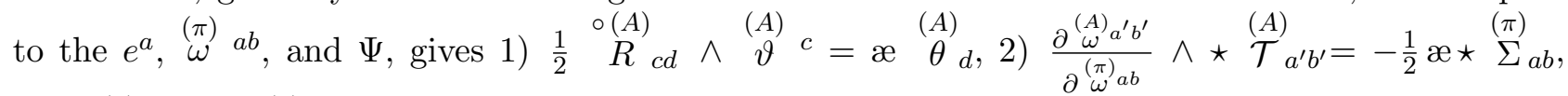
3) $\frac{\delta L_{m}^{(\pi)}}{\delta \Psi}=0, \frac{\delta L_{m}^{(\pi)}}{\delta \bar{\Psi}}=0$.

\subsection{Short-range spin-spin interaction}

In fact, torsion constitutes the more natural and simple way to introduce spin in general relativity. For that reason it is of fundamental importance to see if there are some experiences that indicate, if not directly at least indirectly, the presence of torsion. The most important experiments include neutron interferometry, neutron spin rotation induced by torsion in vacuum, anomalous spin-dependent forces with a polarized mass torsion pendulum, space-based searches for spin in gravity, etc. On the other hand, from the theoretical point of view, a problem of primary importance is to obtain the equation describing the short-range propagating torsion and showing the existence of torsion waves that may contribute a new special polarized effects in these experiments. A broad motivation of the present theoretical work just is the propagating torsion, which, in natural way, can be made a short-range propagating. We then see that it is the $\operatorname{spin} \stackrel{(\pi)}{S}$ and spacetime deformations $\pi(x)$ and $\sigma(x)$ that define the torsion $\stackrel{(A)}{Q}: \stackrel{(A)}{Q} \nu_{\mu \rho}=\stackrel{(A)}{\mathcal{T}}_{\nu}{ }_{\mu \rho}+\frac{1}{2} \delta_{\mu}^{\nu} \stackrel{(A)}{\mathcal{T}}_{\rho \lambda}-\frac{1}{2} \delta_{\rho}^{\nu} \stackrel{(A)}{\mathcal{T}} \lambda_{\mu \lambda}$, which, in turn, defines the Einstein's field tensor $\stackrel{\circ}{G}$. This allows us to define the constraint, which, imposed upon the spacetime deformations $\pi(x)$ and $\sigma(x)$, yields the torsion and spin-spin interaction to be short-range propagating: $\Theta_{\nu^{\prime} \mu \rho}^{\mu^{\prime} \rho^{\prime} \nu}(\pi(x), \sigma(x))=\left(\square+M_{\mathcal{T}}^{2}\right) \stackrel{(\sigma)}{\mathcal{T}}_{\nu}{ }_{\mu \rho}\left(\stackrel{(\sigma)}{\mathcal{T}}{ }^{-1}\right)_{\nu^{\prime}}^{\mu^{\prime} \rho^{\prime}}$, where $\square$ is a generalization of the d'Alembertian operator for covariant derivatives defined on the RC manifold, $U_{4}$. Then, the set of modified Einstein-Cartan equations reduced to $\stackrel{\circ}{G}_{\mu \nu}=æ \stackrel{(\sigma)}{\theta}_{\mu \nu},\left(\square+M_{\mathcal{T}}^{2}\right) \stackrel{(\sigma)}{\mathcal{T}}^{\nu}{ }_{\mu \rho}=-\frac{1}{2} æ \stackrel{(\pi)}{S}^{\nu}{ }_{\mu \rho}$. At large distances $r>\lambda_{\mathcal{T}} \equiv \frac{\hbar}{M_{\mathcal{T}} c}$ (Compton length), torsion vanishes $\stackrel{(\sigma)}{\mathcal{T}}(r)=0$, so in this case the torsion and spin-spin interaction are short-range propagating. To carry through TSSD- $U_{4}$ theory in full generality, below, for example, we may explicitly write the torsionic equation for the Dirac spinor matter source coupled to the metric and to the torsion, both contained implicitly in the connection 
$\left(\stackrel{(\pi)}{\omega} b a_{\mu}\right.$. The spinor connection $\stackrel{(\pi)}{\Gamma}_{\mu}$ is given, up to the addition of an arbitrary vector multiple of I, by the Fock-Ivanenko coefficients: $\stackrel{(\pi)}{\Gamma}_{\mu}=-\frac{1}{4} \stackrel{(\pi)}{\omega}_{a b \mu} \gamma^{a} \gamma^{b}=-\frac{1}{2} \stackrel{(\pi)}{\omega}_{a b \mu} S^{a b}=-\frac{1}{8} e_{c ; \mu}^{\nu}\left[\stackrel{(\pi)}{g}_{\nu}, \gamma^{c}\right]=$ $\frac{1}{8}\left[\stackrel{(\pi)}{g}_{;}^{\nu}, \mu, \stackrel{(\pi)}{g}_{\nu}\right]$, with $S^{a b}=\frac{1}{2} \gamma^{[a} \gamma^{b]}=\frac{1}{4}\left(\gamma^{a} \gamma^{b}-\gamma^{b} \gamma^{a}\right)$ - the spinor representation, where $\gamma^{a}$ are Dirac matrices, and $\stackrel{(A)}{g} \mu=\stackrel{(A)}{e} a^{\mu} \gamma^{a}$. Consequently, the torsionic equation can be recast into the form $\left(\square+M_{\mathcal{T}}^{2}\right) \stackrel{(\sigma)}{\mathcal{T}} \nu_{\mu \rho}=-\frac{i}{4} æ \bar{\psi} \stackrel{(\pi)}{g}[\mu \stackrel{(\pi)}{g} \nu \stackrel{(\pi)}{g} \rho] \psi$, where $\psi$ satisfies the Heisenberg-Ivanenko non-linear equation: $i \stackrel{(\pi)}{g} \stackrel{\rho}{g} \psi_{: \rho}-\frac{3 æ}{8}\left(\bar{\psi} \stackrel{(\pi)}{g} \rho \rho \gamma^{5} \psi\right) \stackrel{(\pi)}{g} \rho \rho \gamma^{5} \psi=m \psi$. This is the Dirac equation written in the so-called second-order formalism, in which the contortion tensor is given explicitly in terms of the spin sources. In the limit when we neglect the usual Riemannian terms depending on the metric and the curvature $\left(\partial_{; \mu} \rightarrow \partial_{\mu}\right)$, as we are interested only in the spin-torsion interaction, we have then $\stackrel{(\sigma)}{\mathcal{T}}_{\mu}{ }_{\mu \rho}(x)=\frac{\mathfrak{x}}{2} \int G_{F}\left(x, x^{\prime}\right) \stackrel{(\pi)}{S} \nu_{\mu \rho}\left(x^{\prime}\right) d^{4} x^{\prime}$ where the Feynman propagator reads $G_{F}\left(x, x^{\prime}\right)=$ $-\frac{1}{4 \pi} \delta(s)+\frac{M_{\mathcal{T}}}{8 \pi \sqrt{s}} H_{1}^{(1)}\left(M_{\mathcal{T}} \sqrt{s}\right)$ if $s \geq 0$; and $G_{F}\left(x, x^{\prime}\right)=-\frac{i M_{\mathcal{T}}}{4 \pi^{2} \sqrt{-s}} K_{1}\left(M_{\mathcal{T}} \sqrt{-s}\right)$ if $s<0$, provided $s=\left(x-x^{\prime}\right)^{2}, H_{1}^{(1)}$ is the Hankel function of first kind and $K_{1}$ is a modified Bessel function. We emphasize that the short-range propagating torsion may contribute a new special polarized effects in the gravitational waves experiments. Neutron interferometry is powerful method to verification of gravitational spin-torsion interaction, whereas it was assumed that the neutron beams (I and II) are polarized in the antiparallel direction to the $z$ axis. In the situation when one (or both) neutron beam(s) interact with torsion the states should be changed, and the interferences of these beams leads to the effect of the polarized rotation plane. So we can observe the effect of the polarized rotation plane due to quantum interferometry, which is caused by the interaction with torsion. At this point we cut short our discussion, and refer the interested reader to original paper (Ter-Kazarian, 2011) for more detail.

\section{The TSSD-metric-affine gravity behind the spacetime deformation}

At low energies the spacetime group associated the matter fields is the Poincaré group (PG). An extension of the Poincaré gauge theory of gravity constructed in the RC geometry, to the most general spacetime symmetry gauge theory, the MAG theory, has the most general type of covariant derivative: in addition to curvature and torsion, the MAG also has nonmetricity, i.e., a nonmetric compatible connection. Hence parallel transport no longer preserves length and angle.

\subsection{Outline of the key points of TSSD-MAG}

Note that there is only indications (but no conclusive evidence) for assuming invariance of physical systems under the action of the entire affine group, and that in MAG one is far from actually calculating $S$-matrix elements. Although the theoretical structure of this theory has been developed, we do not yet much understanding of what new physics is allowed by the MAG theory. One source of improved understanding is exact solutions, and references therein. But due to the highly nonlinear nature of theory, exact solutions are not easily found unless they have a great deal of symmetry. However, to carry in full generality through the extension of TSSD-ideas as applied to more general metricaffine gravity, it reasonable as a next step to gauge immediately the $4+16$ parameter affine group $A(4, R)=R^{4} \otimes G L(4, R)$, which lacks a metric structure altogether and to introduce the metric subsequently. General affine invariance adds dilation and shear invariance as physical symmetries to Poincaré invariance, and both of these symmetries are of physical importance. Dilation invariance is a crucial component of particle physics in the high energy regime. Shear invariance was shown to yield representations of hadronic matter, the corresponding shear current can be related to hadronic quadrupole excitations. From this in the framework of the gauge theory of the affine group with a metric supplemented, as a physically meaningful field theory, it is speculated that the invariance under affine transformations played an essential part at an early stage of the universe, such that todays Poincaré invariance might be a remnant of affine invariance after some symmetry breaking mechanism. 
Thus MAG encompasses the PG as a subcase. A brief outline of the key points of relevance to the context of TSSD can be stated here. To this aim, one enlarges at any point of the base manifold $x \in \widetilde{M}$ a tangent space $T_{x} \widetilde{M}$ to an affine tangent space $A_{x} \widetilde{M}$ by allowing to freely translate elements of $T_{x} \widetilde{M}$ to different points $p \in A_{x} \widetilde{M}$. The collection of all affine tangent spaces $A_{x} \widetilde{M}$ forms the affine bundle $A \widetilde{M}$. An affine frame of $\widetilde{M}$ at $x$ is a pair $\left(\stackrel{(A)}{e}_{a}, p\right.$ ) (where as before $A=\pi, \sigma$ ) consisting of a linear frame $\stackrel{(A)}{e}{ }_{a} \in L_{x} \widetilde{M}$ and a point $p \in A_{x} \widetilde{M}$. The origin of $A_{x} \widetilde{M}$ is that point $o_{x} \in A_{x} \widetilde{M}$ for which the affine frame $\left(\stackrel{(A)}{e}{ }_{a}, o_{x}\right) \in A_{x} \widetilde{M}$ reduces to the linear frame $\stackrel{(A)}{e}_{a} \in L_{x} \widetilde{M}$. The transformation behavior of an affine frame $\left(\stackrel{(A)}{e}{ }_{a}, p\right)$ under an affine transformation $(\Lambda, \tau)$ with $\tau=\tau^{a} \in T^{4} \simeq R^{4}$ and $\Lambda=\Lambda_{a}{ }^{b} \in G L(4, R)$ reads $(\stackrel{(A)}{e}, p) \stackrel{(\Lambda, \tau)}{\longrightarrow}\left(\stackrel{(A)}{e}{ }^{\prime}, p^{\prime}\right)=(\stackrel{(A)}{e} \Lambda, p+\tau)=\left(\stackrel{(A)}{e}_{b} \Lambda_{a}{ }^{b}, p+\tau^{a} \stackrel{(A)}{e}{ }_{a}\right)$. The affine group acts transitively on the affine tangent spaces $A \widetilde{M}$ : Any two affine frames of some $A_{x} \widetilde{M}$ can be related by a unique affine transformation. Consequently, the notion of an affine frame should be enlarged to include all $G L(4, R)$ representations needed. The gauging is accomplished by the introduction of the generalized affine connection as a prescription $\left(\stackrel{(A)}{\Gamma} \stackrel{(L)}{{ }^{\prime}} \stackrel{(A)}{\Gamma}(T)\right.$, which maps infinitesimally neighbouring affine tangent spaces $A_{x} \widetilde{M}, A_{\tilde{x}} \widetilde{M}$, where $\tilde{x}=x+d x$, by an $A(4, R)$-transformation onto each other. The generalized affine connection consists of a $G L(4, R)$-valued 1 -form $\stackrel{(A)}{\Gamma}^{(L)}$ and an $R^{4}$-valued one form $\stackrel{(A)}{\Gamma}^{(T)}$, both of which generate the required $A(4, R)$-transformation. The two affine tangent spaces get now related by an affine transformation according to the prescription $d p=\stackrel{(A)}{\Gamma} \underset{(T) a}{\stackrel{(A)}{e}} \underset{a}{a}, d \stackrel{(A)}{e}{ }_{a}=\stackrel{(A)}{\Gamma} \stackrel{(L) b}{a}_{a}^{(A)} \stackrel{(A)}{e}_{b}$. By introducing origins in $A_{x} \widetilde{M}$, i.e. by soldering $A_{x} \widetilde{M}$ to $\widetilde{M}$, one losts translational invariance in $A_{x} \widetilde{M}$ but gained a local one-to-one correspondence between translations in $A_{x} \widetilde{M}$ and diffeomorphisms on $\widetilde{M}$. However, one can introduce translational invariance by demanding diffeomorphism invariance instead and continue to work with this modified notion of translation invariance. The diffeomorphisms itself, as horizontal transformations in their active interpretation, cannot be gauged according to the usual gauge principle and thus do not furnish their own gauge potential.

\subsection{Lagrangian formulation of the TSSD-MAG}

In the Lagrangian formulation of a general metric-affine theory it was assumed that the matter fields are described in terms of manifields. Actually, the matter fields, denoted in the following by $\psi$, are supposed to be represented by vector- or spinor-valued $p$-forms. However, in MAG one goes beyond Poincaré invariance, assuming that matter fields might not only undergo Poincaré transformations but also the more general linear transformations. In this case, the unavailability of local Lorentz frames poses no problem in the context of boson fields. They are naturally constructed so as to be capable of carrying the action of $S L(4, R)$, instead of the Lorentz group, whether in a local frame or holonomically. However, this is not true of the conventional fermion fields one uses to represent matter, and a linear action should nevertheless be realized, through the use of infinite-component linear field representations of the double-covering of the linear, affine and diffeomorphism groups. More general spinor-representations than the Poincaré-representations have to be constructed. Such representations of the matter fields corresponding to the affine group must exist. Otherwise it does not make sense to demand $A(4, R)$-invariance of a non-vacuum field theory. The construction of the spinor representations of fermionic matter fields in MAG is called manifields. These representations turn out to be infinite dimensional, due to the non-compactness of the gauge (sub-)group $G L(4, R)$. Fermions are then assigned to spinor manifields. The restriction of $G L(4, R)$ to $S O(1, n-1)$ reduces the manifield representations to the familiar spinor representations. The actual gauging of the affine group introduced, in addition to the matter fields $\psi$, the gravitational gauge potentials $\stackrel{(A)}{\Gamma} \stackrel{(T)}{(A)}$ and $\stackrel{(A)}{\Gamma}(L)$. Following the common practice, we will use as gauge potential the translation invariant ${ }_{\vartheta}^{(A)} a$ in place of the translational part $\stackrel{(A)}{\Gamma}(T)$ of the affine connection, simply because it has the immediate interpretation as a reference (co-)frame. Expanded in a holonomic frame, the components of $\vartheta^{(A)} a$ and $\stackrel{(A)}{\Gamma}{ }^{(T)}$ differ just by a Kronecker symbol, as is clear from the definition of $\stackrel{(A)}{\vartheta}^{a}$. Also the homogeneous 
transformation behavior of $\vartheta^{(A)}$ will turn out to be quite convenient. For the action of the $G L(4, R)$ gauge potential, below the shorthand notation $\stackrel{(A)}{\Gamma} a_{a}^{b}$ is used instead of $\left(\stackrel{(A)}{\Gamma}{ }_{c}^{(L) d} L^{c} d\right)_{a}{ }^{b}$. The introduction of a metric into MAG is mandatory since we are interested in a realistic macroscopic gravity theory that contains GR in some limit. So, it was assumed a metric of the general form $\stackrel{(A)}{g}=\stackrel{(A)}{g} \underset{a b}{(A)} \stackrel{(A)}{\vartheta}_{\otimes} \stackrel{(A)}{\vartheta} b$ with coefficients $\stackrel{(A)}{g}_{a b}$ which are independent of the coframe $\stackrel{(A)}{\vartheta}^{a}$. The gauge potentials $\left(\stackrel{(A)}{\vartheta} \stackrel{(A)}{\Gamma} a^{b}\right)$ become true dynamical variables if one has to add to the minimally coupled matter Lagrangian $L_{m}^{(\pi)}$ a gauge Lagrangian $V$. Here, we restrict ourself considering only first order Lagrangian $V$, which must be expressed in terms of the gauge potentials and their first derivatives. The total action reads then $S=$ $\int\left[V\left(\stackrel{(A)}{g}_{a b}, \stackrel{(A)}{\vartheta} a \stackrel{(A)}{N} a b \stackrel{(A)}{T} a \stackrel{(A)}{R}{ }_{a} b\right)+L_{m}^{(\pi)}\left(\stackrel{(A)}{g}_{a b}, \stackrel{(A)}{\vartheta} a\right.\right.$ (fundamentally a representation of the $S L(4, R)$ or of some of its subgroups), its first order Lagrangian $L_{m}^{(\pi)}$ will be embedded in metric-affine spacetime by the minimal coupling procedure, that is, exterior covariant derivatives feature in the kinetic terms of the Lagrangian instead of only exterior ones. Just as ordinary stress is the analogue of the (Hilbert) energy-momentum density, in MAG, one has, in addition, the spin current and the dilation plus shear currents inducing the torsion and nonmetricity fields, respectively. Both spin and dilation plus shear are components of the hypermomentum current, symmetric for dilation plus shear and antisymmetric for spin: spin current $\otimes$ dilation current $\otimes$ shear current. And these currents ought to couple to the corresponding post-Riemannian structures. In accord, the material currents are defined as follows: $\stackrel{(A)}{t} a b=2 \frac{\delta L_{m}^{(\pi)}}{\delta \stackrel{(A)}{g}_{a b}}, \stackrel{(A)}{\theta} a:=\frac{\delta L_{m}^{(\pi)}}{\delta(A)}, \stackrel{(\pi)}{\Delta}{ }_{a}{ }_{b}:=\frac{\delta L_{m}^{(\pi)}}{\delta \stackrel{(\pi)}{\Gamma}{ }_{a} b}$, where ${ }^{(A)}{ }^{a b}$ is the metric (and symmetric) energy-momentum current of matter ('Hilbert current' '), $(A)$

whereas one believes that ${ }^{\theta}$ a should be the dual 3 -form relating to the canonical energy-momentum tensor, $\stackrel{(A)}{\theta} \underset{a}{\mu}$, by $\star \stackrel{(A)}{\theta} \underset{a}{a}=\frac{1}{3 !} \stackrel{(A)}{\theta} \stackrel{\mu}{a}_{a} \varepsilon_{\mu \nu \alpha \beta} \stackrel{(A)}{\vartheta} \nu \alpha \beta$, where we used the abbreviated notations for the wedge product monomials, $\stackrel{(A)}{\vartheta} \mu \nu \alpha \ldots=\stackrel{(A)}{\vartheta} \mu \wedge \stackrel{(A)}{\vartheta} \nu \wedge \stackrel{(A)}{\vartheta} \alpha \wedge \ldots$ and $\star$ denotes the Hodge dual (see Appendix). The canonical energy-momentum density is identical with the dynamical tetrad energymomentum density $e \stackrel{(A)}{\theta} \underset{\mu}{a}=\stackrel{(A)}{\tau} \underset{\mu}{a}$, where $e: \quad=\operatorname{det}\left|e_{a}^{\mu}\right|=\sqrt{-g}$, and that the canonical tensor $e \stackrel{(A)}{\theta}_{\mu \nu}=\stackrel{(A)}{\tau}_{\mu \nu}=\stackrel{(A)}{e}_{a \nu} \stackrel{(A)}{\tau}_{\mu}^{a}$ is generally not symmetric. The relation between the tetrad dynamical energy-momentum tensor and the metric dynamical energy-momentum tensor for matter fields is $(A)$ $(\pi)$

$\theta_{(\mu \nu)}=t_{\mu \nu}$. The canonical hypermomentum current $\stackrel{(\pi)}{\Delta}{ }_{b} b$, which couples to the linear connection, can be decomposed according to $\stackrel{(\pi)}{\Delta}_{a b}=\stackrel{(\pi)}{S}_{a b}+\frac{1}{4} \stackrel{(\pi)}{g}_{a b} \Delta+\widehat{\widehat{\Delta}}_{\alpha \beta}$, with $\stackrel{(\pi)}{S}_{a b}:=\stackrel{(\pi)}{\Delta}_{[a b]}$ as (dynamical) spin current, $\Delta:=\stackrel{(\pi)}{\Delta}^{c}{ }_{c}$, as dilation current, and $\widehat{\Delta}_{a b}$ as symmetric and tracefree shear current, which is a bit more remote from direct observation than the other currents. From variation of a total action, we find the matter and the gauge field equations as follows: $D\left(\frac{\partial L_{m}^{(\pi)}}{\partial(D \Psi)}\right)-(-1)^{p} \frac{\partial L_{m}^{(\pi)}}{\partial \Psi}=$ 0, (matter), D $\left(2 \frac{\partial V}{\partial \stackrel{(A)}{N}_{a b}}\right)+2 \frac{\partial V}{\partial \stackrel{(A)}{g}_{a b}}=-\stackrel{(A)}{t}{ }_{a b}, \quad(0 t h), D\left(\frac{\partial V}{\partial T^{(A)}}\right)+\frac{\partial V}{\partial{ }^{(A)}}=-\stackrel{(A)}{\theta} a$, $\frac{\partial \stackrel{(A)}{\omega} a_{a^{b^{\prime}}}}{\partial \stackrel{(\pi)}{\omega}{ }_{a^{b}}} \wedge\left[D\left(\frac{\partial V}{\partial \stackrel{(A)}{R_{a^{\prime}} b^{\prime}}}\right)+\stackrel{(A)}{\vartheta} a^{\prime} \wedge \frac{\partial V}{\partial T^{(A)} b^{\prime}}+2 \stackrel{(A)}{g} \underset{b^{\prime} c}{ } \frac{\partial V}{\partial \stackrel{(A)}{N}{ }_{a^{\prime} c}}\right]=-\stackrel{(\pi)}{\Delta} a_{b}, \quad(2 n d)$, where we taken into account that the variation of $L_{m}^{(\pi)}$ with respect to the affine connection in MAG is equivalent to the variation with respect to the torsion (or contortion) or spin connection: $\left(\partial \stackrel{(A)}{\omega}_{a^{\prime}} b^{\prime} / \partial \stackrel{(\pi)}{\omega}_{a} b=\partial \stackrel{(A)}{\Gamma} a_{a^{\prime}} b^{\prime} / \partial \stackrel{(\pi)}{\Gamma}{ }_{a}^{b}\right)$. Note that, using the Noether identities, the zeroth field equation can be shown to be redundant, provided the matter equations hold. In first, the canonical energy-momentum of the translational gauge potential $\stackrel{(A)}{\vartheta} a$ can be written in standard form $\left.\left.\frac{\partial V}{\partial \vartheta_{\vartheta}^{(A)}}=\stackrel{(A)}{e} \underset{a}{a}\right\rfloor V-\left(\stackrel{(A)}{e}{ }_{a}\right\rfloor \stackrel{(A)}{T} b^{b}\right) \wedge \frac{\partial V}{\partial \stackrel{(A)}{T} b}-\left(\stackrel{(A)}{e}{ }_{a}\right.$ \rfloor$\left.\left.\stackrel{(A)}{R} b_{b}^{c}\right) \wedge \frac{\partial V}{\partial \stackrel{(A)}{R}_{b}{ }^{c}}-\left(\stackrel{(A)}{\left.e^{(}\right)}{ }_{a}\right\rfloor \stackrel{(A)}{N}{ }_{b c}\right) \frac{\partial V}{\partial \stackrel{(A)}{N}_{b c}}$. Thus, the equations of the standard MAG theory written 
in the framework of the first order Lagrangian, which is expressed in terms of the gauge potentials and their first derivatives, can be recovered for $A=\pi$. Consequently, the (2nd) equation defines a non-dynamical torsion, such that torsion at a given point in spacetime does not vanish only if there is matter at this point, represented in the Lagrangian density by a function which depends on torsion. Unlike the metric, which is related to matter through a differential field equation, torsion does not propagate. However, equations can be equivalently replaced by the set of modified MAG equations for $A=\sigma: D\left(\frac{\partial L_{m}^{(\pi)}}{\partial(D \Psi)}\right)-(-1)^{p} \frac{\partial L_{m}^{(\pi)}}{\partial \Psi}=0, \quad$ (matter), $D\left(2 \frac{\partial V}{\partial \stackrel{(\sigma)}{N}_{a b}}\right)+2 \frac{\partial V}{\partial \stackrel{(\sigma)}{g}_{a b}}=-\stackrel{(\sigma)}{t} \underset{a b}{ }, \quad(0 t h)$, $D\left(\frac{\partial V}{\partial T^{a} a}\right)+\frac{\partial V}{\partial \sigma^{a}}=-\stackrel{(\sigma)}{\theta} a, \quad(1 s t), \Theta_{a^{\prime} b}^{a b^{\prime}}(\pi(x), \sigma(x)) \wedge\left[D\left(\frac{\partial V}{\partial \stackrel{(\sigma)}{R_{a^{\prime}} b^{\prime}}}\right)+\stackrel{(\sigma)}{\vartheta} a^{\prime} \wedge \frac{\partial V}{\partial T^{(\sigma)}}+2 \stackrel{(\sigma)}{g}_{b^{\prime} c} \frac{\partial V}{\partial \stackrel{(\sigma)}{N_{a^{\prime} c}}}\right]=$ $-\stackrel{(\pi)}{\Delta} a_{b}, \quad(2 n d)$, where $\frac{\partial_{\omega_{a^{\prime}}}^{(\sigma)} b^{\prime}}{\partial{\stackrel{(\pi)}{\omega})_{a} b}^{\prime}}:=\Theta_{a^{\prime} b}^{a b^{\prime}}(\pi(x), \sigma(x))$, in which the torsion is dynamical if only $\Theta_{a^{\prime} b}^{a b^{\prime}}(\pi(x), \sigma(x)) \neq \delta_{a}^{a^{\prime}} \delta_{b^{\prime}}^{b}$. Actually, in testing the general MAG equations, we desire, in some limit, to recover the field equation for different (sub-)cases, then we have to put on Lagrange multipliers. Whereas, in the case of TSSD-PG, one has to kill nonmetricity; TSSD-EC is the TSSD-PG with the curvature scalar as gravitational Lagrangian; in the case of TSSD-teleparallel gravity (TSSD-GR ) $_{2}$ in a Weitzenböck spacetime, one has to remove nonmetricity and curvature; and, finally, in the case of GR in a Riemannian space, curvature scalar as Lagrangian, one has to remove nonmetricity and torsion.

Teleparallel gravity. Finally, we will concentrate on the other (sub-)case when $t_{b c}^{a}=\stackrel{(\pi)}{T}_{b c}{ }_{b c}-\stackrel{(\pi)}{C}{ }_{b c}$, and the connection $\stackrel{(\pi)}{\omega} a_{b c}$ vanishes, which characterizes teleparallel gravity. In this case, the field
equations can be derived from the least action, $\delta \dot{S}=0$, with the Lagrangian of teleparallel gravity, where we take Lagrange multipliers for extinguishing nonmetricity, curvature, which holds when the deformation $\sigma(x)$ vanishes identically $(\sigma(x) \equiv 0): V_{\|}=-\frac{1}{4 æ} \dot{T}^{a} \wedge^{*}{ }^{*}{ }^{(1)} \dot{T}_{a}+2^{(2)} \dot{T}_{a}+\frac{1}{2}{ }^{(3)} \dot{T}_{a}$ )$+\frac{1}{2} \dot{N}_{a b} \wedge^{(1)} \lambda^{a b}+\dot{R}_{a}{ }^{b} \wedge{ }^{(2)} \lambda^{a}{ }_{b}$, where the three irreducible pieces of the torsion $\stackrel{(A)}{T}^{a}={ }^{(1)} \stackrel{(A)}{T}^{(A)}$

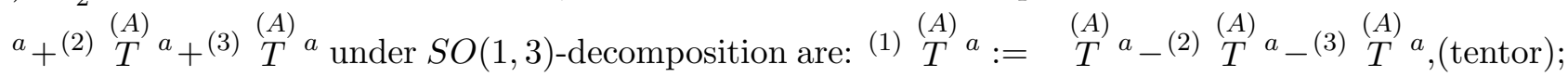
(2) $\left.\stackrel{(A)}{T} a:=\frac{1}{3} \stackrel{(A)}{\vartheta} a \wedge\left(\stackrel{(A)}{e}_{b}\right\rfloor \stackrel{(A)}{T} b\right)$, (trator); ${ }^{(3)} T^{a}:=-\frac{1}{3} *\left(\stackrel{(A)}{\vartheta} a \wedge *\left(\stackrel{(A)}{T}^{b} \wedge \stackrel{(A)}{\vartheta} b\right)\right.$ ), (axiator). The projective transformation $\stackrel{(A)}{\Gamma} a^{b} \longrightarrow \stackrel{(A)}{\Gamma} a^{b}+\delta_{a}^{b} P$, with arbitrary 1-form field $P$, leaves the Hilbert-Einstein type Lagrangian invariant, which leads to the connection determined up to a 1-form. Projectively related connections have the same (unparametrized) geodesics. So, only projectively invariant matter Lagrangians would be allowed. This necessitates to abandon this constraint, at the very least replacing semi-Riemann geometry by Weyl's. Namely, to remove this constraint from the gravitational Lagrangians, we may lift the Lagrange multiplier $\lambda^{a b}$ and add a dilaton type massless scalar field to $V^{\prime}=V+\frac{1}{2} d \Phi \wedge \star d \Phi$, in the context of the Weyl 1-form $\stackrel{(A)}{N}$, which is of the type of a

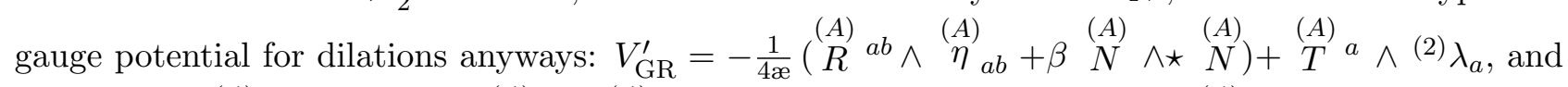
$V_{\mathrm{EC}}^{\prime}=-\frac{1}{4 \nsim}\left(\stackrel{(A)}{R}^{a b} \wedge \stackrel{(A)}{\eta}_{a b}+\beta \stackrel{(A)}{N} \wedge \star \stackrel{(A)}{N}\right)$, respectively. Provided, the trace $\stackrel{(A)}{\Gamma}_{c} c$ of a connection is closely related to the Weyl 1-form, as $\stackrel{(A)}{\Gamma} c_{c} c=2 \stackrel{(A)}{N}+d \ln \sqrt{\left|\operatorname{det} \stackrel{(A)}{g}_{a b}\right|}, d \stackrel{(A)}{\Gamma} c_{c}^{c}=\stackrel{(A)}{R} c_{c}^{c}=2 d \stackrel{(A)}{N}$. In case if matter is present and supplies energy-momentum and hypermomentum currents, then the hypermomentum, via the second field equation, turns out to be proportional to the post-Riemannian pieces of the connection.

In case of translational gauge with the most general term $V=d \stackrel{(A)}{\vartheta} a \wedge \stackrel{(A)}{H}$, quadratic in $\stackrel{(A)}{\vartheta} a$,

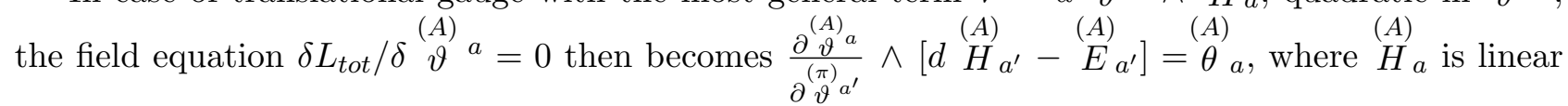
in $d \stackrel{(A)}{\vartheta} a$, and $\left.\left.\left.\stackrel{(A)}{E}{ }_{a}=\left(\stackrel{(A)}{e}_{a}\right\rfloor d \stackrel{(A)}{\vartheta} b\right) \wedge \stackrel{(A)}{H}{ }_{b}-\frac{1}{2} \stackrel{(A)}{e}_{a}\right\rfloor(d \stackrel{(A)}{\vartheta} b) \wedge \stackrel{(A)}{H} b_{b}\right)=\frac{1}{2}\left[\left(\stackrel{(A)}{e}_{a}\right\rfloor d \stackrel{(A)}{\vartheta} b\right) \wedge \stackrel{(A)}{H} b_{b}-d \stackrel{(A)}{\vartheta}$ $\left.\left.b \wedge\left(\stackrel{(A)}{e}_{a}\right\rfloor \stackrel{(A)}{H} b\right)\right]$, the energy-momentum current of the gauge field. This can be considered as a starting 
point for turning to the Lagrangians with the quadratic ansatz for the kinetic term, i.e. quadratic in the field strengths, which would be an interesting topic not discussed in this paper. For more detail see (Ter-Kazarian, 2015).

\section{An extended phase space stochastic quantization of constrained hamiltonian systems}

In this section, we give a theory of the extended phase space stochastic quantization of constrained hamiltonian systems (Ter-Kazarian \& Sobouti, 2008).

Objectives. The concept of an 'extended' Lagrangian, $\mathcal{L}(p, q, \dot{p}, \dot{q})$ in phase space allows a subsequent extension of Hamilton's principle to actions minimum along the actual trajectories in $(p, q)-$, rather than in $q$-space. The following notational conventions are used throughout this paper: $\dot{p}$ denotes $d p / d x_{0}$ and so on, where $x_{0}$ is the real time. This extension, in turn, allows a definition of "second" momenta $\pi_{p}=\delta \mathcal{L} / \delta \dot{p}$ and $\pi_{q}=\delta \mathcal{L} / \delta \dot{q}$, and a subsequent introduction of an "extended" phase space $\left(p, q, \pi_{p}, \pi_{q}\right)$ and of an "extended" Hamiltonian, $\mathcal{H}\left(p, q, \pi_{p}, \pi_{q}\right)$. This simple formalism manifests its practical and technical virtue in the proposed canonical quantization in $(p, q)$ space that at once provides a framework for quantum statistical mechanics, for the classical statistical mechanics (Liouville's equation), for the conventional quantum mechanics as a special case, for von Neumann's density matrix and its equation of evolution as its inevitable corollaries. Wigner's distributions and the equation satisfied by them are also obtained by an appropriate canonical transformation in the proposed $\left(p, q, \pi_{p}, \pi_{q}\right)$-space.

Ordering of $p$ and $q$ factors in conventional quantum mechanics has always been a matter of debate. For, there is nothing in the basic postulates of quantum mechanics to decide on the issue. On the other hand the phase space quantization is constructed on the premises that $p$ and $q$ are independent variables. Thus, in reducing the theory to that of Schrödinger and/or Heisenberg, the standard ordering emerges as the rule of game. For example, $q p$ in $q$-representation and $p q$ in $p$-representation. For Wigner's distributions the appropriate ordering is the symmetric one. For example, $\frac{1}{2}(p q+q p)$ instead of $p q$ or $q p$. This ordering is also obtained from the standard ordering by the same canonical transformation which transforms the state functions and evolution equations to those of Wigner.

Since Wigner's initial attempt, 1932, alternative phase space distributions have been proposed. Of these alternatives, the ones compatible with the uncertainty principle are obtainable from that by suitable canonical transformation in $\left(p, q, \pi_{p}, \pi_{q}\right)$-space. Husimi's all-positive distributions are, however, exceptions. For, his averaging of Wigner's distributions over small cells around phase space points makes the averaged distributions incompatible with the uncertainty principle.

The stochastic quantization method (SQM) of recent years is an alternative to the conventional canonical and path-integral quantizations. Conceptually and techniquewise it is versatile and powerful. Our interest here is to generalize SQM, to study the classical stochastic processes underlying the phase space quantization. In its present formulation, SQM exploits well-defined Markoffian process of Wiener's type with Gaussian white-noise. One may, however, envisage that different stochastic processes with respect to a fictitious time may yield different variations of quantum theories.

\subsection{An Extended Phase Space Formulation of SQM}

Consider a dynamical system with $\mathrm{N}$ degrees of freedom described by the $2 \mathrm{~N}$ coordinates $q=$ $\left(q_{1}, \ldots q_{N}\right)$ and momenta $p=\left(p_{1}, \ldots p_{N}\right)$ and a Lagrangian $\mathcal{L}^{q}(q, \dot{q})$ in $q$-representation and the corresponding $\mathcal{L}^{p}(p, \dot{p})$ in $p$-representation. In general, $\mathcal{L}^{q}$ and $\mathcal{L}^{p}$ are the Fourier transforms of each other. One may now defines an extended Hamiltonian $\mathcal{H}\left(p, q, \pi_{p}, \pi_{q}\right)=\sum_{n=1} \frac{1}{n !}\left\{\frac{\partial^{n} H}{\partial p^{n}} \pi_{q}^{n}-\frac{\partial^{n} H}{\partial q^{n}} \pi_{p}^{n}\right\}$, where $H(p, q)=p_{i} \dot{q}_{i}-\mathcal{L}^{q}=q_{i} \dot{p}_{i}-\mathcal{L}^{p}$ is the conventional Hamiltonian of the system. Introducing an imaginary time $x_{4}=i x_{0}$, we define the Euclidian extended action as $S\left[p, q, \pi_{p}, \pi_{q}\right]=$ $\int\left[-i \pi_{p_{i}} \frac{d p_{i}}{d x_{4}}-i \pi_{q_{i}} \frac{d q_{i}}{d x_{4}}+\mathcal{H}\left(p, q, \pi_{p}, \pi_{q}\right)\right] d x_{4}$. Following a general prescription of SQM, in case at hand the Parisi-Wu ansatz consists of proposing a Markoffian hypothetical stochastic process by the following set of Langevin equations: $\frac{d q_{i}}{d t}=-\frac{\delta S}{\delta q_{i}}+\xi_{i}^{q}(t), \quad \gamma \frac{d \pi_{q_{i}}}{d t}=-\frac{1}{\gamma} \frac{\delta S}{\delta \pi_{q_{i}}}+\eta_{i}^{q}(t), \quad \gamma \frac{d p_{i}}{d t}=$ 
A new look at some aspects of geometry, particle physics, inertia, radiation and cosmology $-\frac{1}{\gamma} \frac{\delta S}{\delta p_{i}}+\eta_{i}^{p}(t), \quad \frac{d \pi_{p_{i}}}{d t}=-\frac{\delta S}{\delta \pi_{p_{i}}}+\xi_{i}^{p}(t)$, where an additional "fictitious time" $t$ is introduced, the $\xi_{i}^{\nu}(t)$ and $\eta_{i}^{\nu}(t)(\nu=q, p)$ are Gaussian white-noise sources with $<\xi_{i}^{\nu}(t), \xi_{j}^{\nu^{\prime}}\left(t^{\prime}\right)>=2 \delta_{i j} \delta_{\nu \nu^{\prime}} \delta\left(t-t^{\prime}\right), \quad<$ $\eta_{i}^{\nu}(t), \eta_{j}^{\nu^{\prime}}\left(t^{\prime}\right)>=2 \delta_{i j} \delta_{\nu \nu^{\prime}} \delta\left(t-t^{\prime}\right), \quad<\xi_{i}^{\nu}(t), \eta_{j}^{\nu^{\prime}}\left(t^{\prime}\right)>=0$, and $\gamma$ is an arbitrary dimensional parameter. In this, we have only to look upon the fictitious time $t$ as a mathematical tool, but need not to find its physical meaning. A remark on notation: Functional dependencies on variables are indicated by square brackets, such as $S[p, q, \ldots]$. The formalism being followed is based on a well-defined classical Wiener-Markoffian process. The Gaussian white-noises incorporated into equations above are designed to yield the quantum mechanics as its thermal equilibrium limit. Therefore, the task is to show that the dynamical system has an equilibrium distribution equivalent to the conventional path-integral measure. The procedure is (a) to define a Fokker-Planck Lagrangian, (b) to define Fokker-Planck momenta from this Lagrangian, (c) to compose a FokkerPlanck Hamiltonian, and finally (d) to set up the Fokker-Planck equation for the distribution of the system in the extended phase space. For example, the Fokker-Planck Hamiltonian is: $\mathcal{H}^{F}=$ $\pi_{q_{i}}^{F} \frac{d q_{i}}{d t}+\pi_{\pi_{q_{i}}}^{F} \frac{d \pi_{q_{i}}}{d t}+\pi_{p_{i}}^{F} \frac{d p_{i}}{d t}+\pi_{\pi_{p_{i}}}^{F} \frac{d \pi_{p_{i}}}{d t}-\mathcal{L}^{F}$, provided, the Fokker-Planck equation for the probability distribution $\Phi\left[p, q, \pi_{p}, \pi_{q}, t\right]$ is $\frac{\partial}{\partial t} \Phi\left[p, q, \pi_{p}, \pi_{q}, t\right]=\mathcal{H}^{F} \Phi\left[p, q, \pi_{p}, \pi_{q}, t\right]$. Here we have replaced the canonical momenta $\pi_{q_{i}}^{F}, \pi_{\pi_{q_{i}}}^{F}, \pi_{p_{i}}^{F}$ and $\pi_{\pi_{p_{i}}}^{F}$ with $-\partial / \partial q_{i},-\partial / \partial \pi_{q_{i}},-\partial / \partial p_{i}$ and $-\partial / \partial \pi_{p_{i}}$, respectively. Then the equilibrium distribution clearly reads $\Phi\left[p, q, \pi_{p}, \pi_{q}\right] \propto \exp \left(-S\left[p, q, \pi_{p}, \pi_{q}\right]\right)$. Thus, the Langevin equation give the same result as the conventional path-integral quantization method in extended phase space if only the drift forces $K_{i}(p, \ldots t)=-\left(\frac{\delta S[p, \ldots]}{\delta p_{i}}\right)_{p=p\left(x_{0}, t\right)}$, etc., have a damping effect. Along the trajectories in $(p, q)$ space, however, it produces the state functions, $\chi\left(p, q, x_{0}\right)$ : $i \hbar \frac{\partial}{\partial x_{0}} \chi=\mathcal{H} \chi$. Solutions are $\chi=a_{\alpha \beta} \psi_{\alpha}\left(q, x_{0}\right) \phi_{\beta}^{*}\left(p, x_{0}\right) e^{-i p q / \hbar}, a=a^{\dagger}, \quad$ positive definite, $\operatorname{tr} a=$ 1 , where summation over repeated indices is implied, and $\psi_{\alpha}$ and $\phi_{\alpha}^{*}$ are solutions of the conventional Schrödinger equation in $q$ - and $p$-representations, respectively. They are mutually Fourier transforms $\psi_{\alpha}\left(q, x_{0}\right)=(2 \pi \hbar)^{-N / 2} \int \phi_{\alpha}\left(p, x_{0}\right) e^{i p q / \hbar} d p, \phi_{\alpha}\left(p, x_{0}\right)=(2 \pi \hbar)^{-N / 2} \int \psi_{\alpha}\left(q, x_{0}\right) e^{-i p q / \hbar} d q$. Note that the $\alpha$ and $\beta$ are not, in general, eigenindices. The normalization condition for $\chi$, is $\int \chi d p d q=\operatorname{tr}(a)=1$.

\subsection{Stochastic Quantization of Extended Dynamical Systems With Constraints}

Here we discuss the SQM of an extended dynamical system with $M$ first class independent and irreducible constraints $\phi^{a}\left(p, q, \pi_{p}, \pi_{q}\right)=0, \quad a=1,2, \ldots, M<N$. For reasons of simplicity let there also be $M$ gauge conditions: $\chi^{a}\left(p, q, \pi_{p}, \pi_{q}\right)=0, \quad a=1,2, \ldots, M$. Equations obtained in previous subsection define a $(4 N-2 M)$ dimensional submanifold in phase space on which the system orbits dwell. For convenience we introduce the following new variables: $x_{q_{i}}=\left(q_{1}, \ldots, q_{N}, \pi_{p_{1}}, \ldots, \pi_{p_{N}}\right)$, $x_{p_{i}}=\left(p_{1}, \ldots, p_{N}, \pi_{q_{1}}, \ldots, \pi_{q_{N}}\right)$. The gauge conditions are such that $\operatorname{det} \Delta^{a b} \neq 0$, where $\Delta^{a b}$ is the Poisson bracket of $\chi^{a}$ and $\phi^{b} \Delta^{a b}=\frac{\partial \chi^{a}}{\partial x_{q_{i}}} \frac{\partial \phi^{b}}{\partial x_{p_{i}}}-\frac{\partial \chi^{a}}{\partial x_{p_{i}}} \frac{\partial \phi^{b}}{\partial x_{q_{i}}}$. The Euclidean path-integral measure for such a system can be obtained by the quantization procedure. The Faddeev-Popov path-integral formula for this system is $\langle f \mid i\rangle=\frac{1}{2 N} \int \mathcal{D} x_{q_{i}} \mathcal{D} x_{p_{i}} \delta\left(\phi^{a}\right) \delta\left(\chi^{a}\right) \operatorname{det} \Delta^{a b} \exp \left(-S\left[x_{p}, x q\right]\right)$, where $S\left[x_{p}, x_{q}\right]$ is the extended Euclidian action. Our major goal is now to reproduce this equation from the standpoint of SQM in phase space in thermal equilibrium limit. Next we attempt to write the derived equations in a covariant form; that is, in a form invariant under general coordinate transformations. In this, we introduce the notation $\left(x^{I}\right)=\left(x_{I}\right)=\left(x_{q i}, x_{p i}\right),\left(X^{I}\right)=\left(Q^{a}, P^{a}, Q^{\alpha}, P^{\alpha}\right), I=1, \ldots, 4 N$. Hereafter, the following convention will be observed in indexing the new variables. To begin with, the $x$-coordinates are Euclidean ones. It will not matter if they are indexed covariantly or contravariantly. The $X$-coordinates, on the other hand, are curvilinear ones. A contravariant index could be lowered by an appropriate metric tensor to be introduced shortly. The manifold $M^{4 N}$ spanned by $X$ - coordinates could be split into two submanifolds $M^{2 M}$ and $M^{4 N-2 M}$. The $X$-coordinates spanning $M^{2 M}$ will be indexed by $A, B, \ldots=1, \ldots, 2 M$. Those spanning $M^{4 N-2 M}$ will be indexed by $\Lambda, \Sigma, \ldots=2 M+1, \ldots, 4 N$. The indices $I, J, \ldots$, will be reserved for the whole manifold $M^{4 N}$. The con-

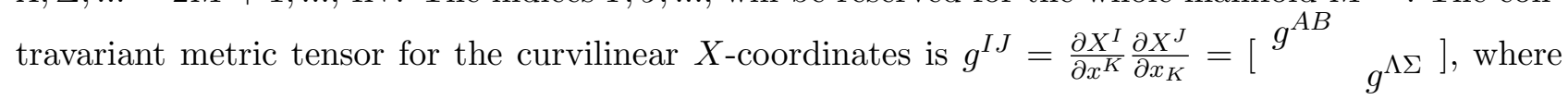
the $2 M \times 2 M$ tensor $g^{A B}$ is $g^{A B}=\frac{\partial X^{A}}{\partial x^{K}} \frac{\partial X^{B}}{\partial x_{K}}=\left[\begin{array}{ll}\left\{\phi^{a}, \phi^{b}\right\} & \left\{\phi^{a}, \chi^{b}\right\} \\ \left\{\chi^{a}, \phi^{b}\right\} & \left\{\chi^{a}, \chi^{b}\right\}\end{array}\right]$. The Poisson brackets, here, 
are to be calculated in $\left(x_{q_{i}}, x_{p_{i}}\right)$-coordinates, For conventional gauge conditions one has $\left\{\chi^{a}, \chi^{b}\right\}=0$. The Laplace expansion of det $g^{A B}$ then gives $\operatorname{det} g^{A B}=-\operatorname{det}\left\{\phi^{c}, \chi^{d}\right\} \operatorname{det}\left\{\chi^{e}, \phi^{f}\right\}=\operatorname{det}\left\{\phi^{c}, \chi^{d}\right\}^{2}$, $\operatorname{det} g_{A B}=\operatorname{det}\left\{\phi^{c}, \chi^{d}\right\}^{-2}$. The expression for $g^{\Lambda \Sigma}$ is $g^{\Lambda \Sigma}=\frac{\partial X^{\Lambda}}{\partial x^{K}} \frac{\partial X^{\Sigma}}{\partial x_{K}} ; \quad \Lambda, \Sigma=2 M+1, \cdots, 4 N$. For the present, there is no need to manipulate $g^{\Lambda \Sigma}$ beyond its definition. We are now in a position to write down the Langevin equations in manifest covariant forms. Then $\frac{d X^{\Lambda}}{d x^{I}} \frac{d X^{A}}{d x_{I}}=0$, and $\frac{d X^{A}}{d t}=0$. This gives $\frac{d X^{\Lambda}}{d t}=-g^{\Lambda \Sigma} \frac{\delta \widetilde{S}[X]}{\delta X^{\Sigma}}+\frac{\partial X^{\Lambda}}{d x^{I}} \zeta^{I}$, where $\zeta^{I}=\left(\xi^{i}, \eta^{j}\right)$, and $\widetilde{S}[X]=S[x(X)]$, is the action integral written in $X$-coordinates. This equation contains no new information, beyond the fact that $\phi^{a}, \chi^{a}$ are to vanish along the phase space trajectories. Finally the form invariant Fokker-Planck equation emerges as $\frac{\partial \widetilde{\Phi}\left[X^{\Lambda}, t\right]}{\partial t}=\frac{1}{\sqrt{\operatorname{det} g^{\Lambda \Sigma}}} \frac{\partial}{\partial X^{\Lambda}}\left[\sqrt{\operatorname{det} g^{\Lambda \Sigma}} g^{\Lambda \Sigma}\left(\frac{\partial}{\partial X^{\Sigma}}+\frac{\delta \widetilde{S}[X]}{\delta X^{\Sigma}}\right) \widetilde{\Phi}\left[X^{\Lambda}, t\right]\right]$. The stationary solution is $\widetilde{\Phi}_{e q}\left[X^{\Lambda}\right]=\frac{1}{\sqrt{\operatorname{det} g^{\Lambda \Sigma}}} \exp \left(-\widetilde{S}\left[X^{\Lambda}\right]\right)$. In the limit of thermodynamic equilibrium the probability of finding the system on the constraint surface in the volume element $d^{(4 N-2 M)} X$ centered at $X^{\Lambda}$ is $\frac{1}{\sqrt{\operatorname{detg}^{\Lambda \Sigma}}} \exp \left(-\widetilde{S}\left[X^{\Lambda}\right]\right) d^{(4 N-2 M)} X=\frac{1}{\sqrt{\operatorname{det} g^{\Lambda \Sigma}}} \exp \left(-\widetilde{S}\left[X^{I}\right]\right) \delta^{(2 M)}\left(X^{A}\right) d^{(4 N)} X$. Where $\delta^{(2 M)}\left(X^{A}\right)$ is the Dirac delta function in $M^{2 M}$. It is introduced to ensure that the system stays on the constraint surface. To transform back to the Euclidean coordinates $\left\{x^{I}\right\}$ we note that $\widetilde{S}[X]$ transform into $S[x]$, and the volume element $d^{(4 N)} X$ transforms into $\sqrt{\operatorname{det} g^{I J}} d^{(4 N)} x$, where $\operatorname{det}\left(g^{I J}\right)=\operatorname{det}\left(g^{A B}\right) \operatorname{det}\left(g^{\Lambda \Sigma}\right)$. Hence $\Phi_{e q}\left[x_{p}, x_{q}\right]=\operatorname{det}\left\{\chi^{a}, \Phi^{b}\right\} \delta^{(M)}\left(\chi^{c}\right) \delta^{(M)}\left(\Phi^{d}\right) \exp \left(-S\left[x_{p}, x_{q}\right]\right)$. Thus, one of the most remarkable features of SQM is that one may quantize even dynamical systems with non-holonomic constraints as it is seen in the case of the stochastic gauge fixing.

\section{An extended phase space SUSY quantum mechanics}

In this section, we will concern ourselves with the extended phase space quantum mechanics of particles which have both bosonic and fermionic degrees of freedom (Ter-Kazarian, 2009), i.e., the quantum field theory in $(0+1)$-dimensions in $q-$ (position) and $p-$ (momentum) spaces, exhibiting supersymmetry. We present $(\mathrm{N}=2)$-realization of the supersymmetry algebra, and discuss the vacuum energy and the topology of super-potentials. To demonstrate practical merits of shape-invariance of exactly solvable extended SUSY potentials which has underlying algebraic structure, as an application we obtain analytic expressions for the entire energy spectrum of extended Hamiltonian with Scarf potential without ever referring to underlying differential equation.

\subsection{Extended phase space SUSY algebra}

Practically and techniquewise the conventional SUSY method is versatile and powerful. Following a general prescription of SUSY quantum mechanics, we call an extended phase space quantum mechanical system characterized by an extended Hamiltonian $H_{\text {ext }}$ acting in some Hilbert space $\mathcal{H}$ supersymmetric if there exist selfadjoint nilpotent operators $Q_{i}=Q_{i}^{\dagger}, \quad i=1,2, \ldots, N$, called supercharges, which also act on states in $\mathcal{H}$ and fulfill the following SUSY algebra: $\left\{Q_{i}, Q_{j}\right\}=Q_{i} Q_{j}+Q_{j} Q_{i}=$ $2 H_{\text {ext }} \delta_{i j},\left[Q_{i}, Q_{j}\right]=Q_{i} Q_{j}-Q_{j} Q_{i}=0, i, j=1, \ldots, N$. Pursuing the analogy with these ideas in outlined here approach let a selfadjoint operator $P=P^{\dagger}$ be Witten operator or Witten parity, which anticommutes with the supercharges, and therefore commutes with an extended Hamiltonian, and whose square is equal to the identity $\left\{Q_{i}, P\right\}=0,\left[H_{e x t}, P\right]=0, P^{2}=1$. This operator allows to introduce the notion of bosonic and fermionic states independently of an underlying space-time symmetry. The Witten parity can also be written in the form $P=(-1)^{n_{F}}$ where $n_{F}$ is the fermionnumber operator. Therefore, eigenstates of $P$ with eigenvalue -1 correspond to fermions and those with +1 correspond to bosons. In accordance, the bosonic $\mathcal{H}_{B^{-}}$and fermionic $\mathcal{H}_{B^{-}}$subspaces read $\mathcal{H}_{B}=\{\chi \in \mathcal{H} \mid P \chi=+\chi\}, \mathcal{H}_{F}=\{\chi \in \mathcal{H} \mid P \chi>=-\chi\}$. Hence, any state $\chi \in \mathcal{H}$ can be decomposed into its bosonic and fermionic components as follows: $\chi=\left(\begin{array}{c}\chi_{B} \\ \chi_{F}\end{array}\right)$. The Hilbert space may be written as a product space $\mathcal{H}=\mathcal{H}_{0} \otimes \mathcal{C}^{2}$, and thus, the Witten operator is represented by the third Pauli matrix $\sigma_{3}: P=\mathbf{1} \otimes \sigma_{3}=\left(\begin{array}{cc}1 & 0 \\ 0 & -1\end{array}\right)$. It will be more appropriate to use the notion spin-up and spin- 
down states (of a fictitious spin- $\frac{1}{2}$-particle with mass $m>0$ moving along the d-dimensional Euclidean line $\mathcal{R}^{d}$ line) instead of bosonic and fermionic states, respectively. Having in addition only cartesian degrees of freedom $\mathcal{H}_{0}$ is given by the space of square-integrable functions over the $d$-dimensional Euclidean space $\mathcal{R}^{d}, \quad \mathcal{H}_{0}=\mathcal{L}^{2}\left(\mathcal{R}^{d}\right) \otimes \mathcal{L}^{2}\left(\mathcal{R}^{d}\right), \quad d \in \mathbf{N}$. The SUSY has also implications on the spectral properties of the extended hamiltonian $H_{\text {ext }}$. First of all, we note $H_{\text {ext }}=Q_{i}^{2} \geq 0$. That is, the extended Hamiltonian has only non-negative eigenvalues. Suppose that $\chi_{r}$ is an eigenstate of $H_{\text {ext }}$ with positive eigenvalue $E_{r}>0$. Then it follows immediately from the algebra that $\widetilde{\chi_{r}}(q, p)=\frac{1}{\sqrt{E_{r}}} Q_{i} \chi_{r}(q, p)$, $i=1,2 \ldots, N$, is also an eigenstate with the same positive eigenvalue. Hence, all positive-energy eigenstates occur in spin-up (boson) spin-down (fermion) pairs. Actually, a multiplicity of degeneracy of the levels of Hamiltonian $H_{\text {ext }}$ with the energy $E$ equals to a dimension of invariant subspace with respect to the action of all the $Q_{i}$. If $E=0$, then the corresponding subspace is one-dimensional - a level of zero point energy. In general, the superalgebra defines the Clifford algebra with the basis of $q_{i}=\frac{Q_{i}}{\sqrt{E}}$ for non-zero-energy levels of $H_{e x t}$, which is a key point in the SUSY theories. Due to it a definition of the multiplicity of degeneracy of the energy levels reduced to a definition of a dimension of the representations of the Clifford algebra, which is well-known. For the even and odd number $N$ a dimension of the representation of Clifford algebra is given as $\nu=2^{n}=2^{[N / 2]}$, where [...] means the integer part, namely the $\nu$ defines a number of states in given supermultiplet, Thus, the major law for the supermultiplets is that each of them contains an equal number of fermionic and bosonic degrees of freedom $n_{B}=n_{F}$. Certainly, re-writing the parity operator in the form $(-1)^{2 S}$ where $S$ is the spin angular momentum having eigenvalue +1 acting on a bosonic state and eigenvalue -1 acting on a fermionic state, we get $\sum_{i}<i\left|(-1)^{2 S} H_{\text {ext }}\right| i>=\sum_{i}<i\left|(-1)^{2 S} Q Q^{\dagger}\right| i>+\sum_{i}<i \mid$ $(-1)^{2 S} Q^{\dagger} Q\left|i>=\sum_{i}<i\right|(-1)^{2 S} Q Q^{\dagger}\left|i>-\sum_{j}<j\right|(-1)^{2 S} Q Q^{\dagger} \mid j>=0$. Here one has used the relation of completeness $\S_{i}|i><i|=1$ within the subspace of states invariant with respect to the action of $Q$ and $Q^{\dagger}$, and the fact that the operator $(-1)^{2 S}$ must anticommute with $Q$. The $\S_{i}<i\left|(-1)^{2 S} H_{\text {ext }}\right| i>=E \operatorname{tr}\left[(-1)^{2 S}\right]$ is proportional to the number of bosonic degrees of freedom $n_{B}$ minus the number of fermionic degrees of freedom $n_{F}$ in the trace. Hence, this relation holds for any $E>0$ in each supermultiplet. This, however, is in general not true for possible zero-energy eigenstates. If the groundstate energy of $H_{\text {ext }}$ is equal to zero, that is, exists a state $\chi_{0} \in \mathcal{H}_{0} \otimes \mathcal{C}^{2}$ such that $H_{\text {ext }} \chi_{0}=0$, then SUSY is said to be a good symmetry, i.e. the groundstate is invariant under SUSY transformations $Q_{i} \chi_{0}=0$. If the groundstate energy of $H_{\text {ext }}$ is strictly positive then SUSY is said to be broken.

\subsection{The $(\mathrm{N}=2)-\mathrm{SUSY}$ in extended phase space}

In constructing a particular $(\mathrm{N}=2)$-realization of the SUSY algebra in the Hilbert space $\mathcal{H}=$ $\mathcal{H}_{0} \otimes \mathcal{C}^{2}=\left[\mathcal{L}^{2}(\mathcal{R}) \otimes \mathcal{L}^{2}(\mathcal{R})\right] \otimes \mathcal{C}^{2}$, let us first introduce a bosonic operators $B_{ \pm}$in $q$ - and $p$ representations and fermionic operator $\hat{f}: \quad B_{q \mp}: \mathcal{L}^{2}(\mathcal{R}) \rightarrow \mathcal{L}^{2}(\mathcal{R}), \quad B_{q \mp}=\left[p+\pi_{q} \pm i W(q)\right]$, $B_{p \mp}: \mathcal{L}^{2}(\mathcal{R}) \rightarrow \mathcal{L}^{2}(\mathcal{R}), \quad B_{p \mp}=\left[q+\pi_{p} \pm i V(p)\right]$, and $\hat{f}: \mathcal{C}^{2} \rightarrow \mathcal{C}^{2}, \hat{f}=\frac{1}{2}\left[\hat{\psi}_{+}, \hat{\psi}_{-}\right]$. The $\left(\hat{\pi}_{q}, \hat{q}\right)$ and $\left(\hat{\pi}_{p}, \hat{p}\right)$ are usual bosonic momentum and coordinate operators respectively in $q$ - and $p$ - spaces, while $\hat{\psi}_{ \pm}$are two real fermionic creation and annihilation nilpotent operators describing the fermionic variables, $W(q): \mathcal{R} \rightarrow \mathcal{R}$ and $V(p): \mathcal{R} \rightarrow \mathcal{R}$ are the piecewise continuously differentiable functions called SUSY potentials. The $\hat{\psi}_{ \pm}$, having anticommuting c-number eigenvalues, imply $\hat{\psi}_{ \pm}=\sqrt{\frac{1}{2}}\left(\hat{\psi}_{1} \pm i \hat{\psi}_{2}\right)$, $\left\{\hat{\psi}_{\alpha}, \hat{\psi}_{\beta}\right\}=\delta_{\alpha \beta},\left\{\hat{\psi}_{+}, \hat{\psi}_{-}\right\}=1, \hat{\psi}_{ \pm}^{2}=0$. They can be represented by finite dimensional matrices $P=\mathbf{1} \otimes \sigma_{3}$, where $\sigma^{ \pm}=\frac{\sigma_{1} \pm \sigma_{2}}{2}$ are the usual raising and lowering operators for the eigenvalues of $\sigma_{3}$. The fermionic operator commutes with the $H_{\text {ext }}$ and is diagonal in this representation with conserved eigenvalues $\pm \frac{1}{2}$. Due to it the wave functions become two-component objects: $\chi(q, p)=$ $\left(\begin{array}{c}\chi_{+1 / 2}(q, p) \\ \chi_{-1 / 2}(q, p)\end{array}\right)=\left(\begin{array}{c}\chi_{1}(q, p) \\ \chi_{2}(q, p)\end{array}\right)=\left(\begin{array}{l}\psi_{1}(q) \phi_{1}(p) \\ \psi_{2}(q) \phi_{2}(p)\end{array}\right)$, where the states $\psi_{1,2}(q), \phi_{1,2}(p)$ correspond to fermionic quantum number $f= \pm \frac{1}{2}$, respectively, in $q-$ and $p-$ spaces. Let us now to deal with abstract space of eigenstates of the conjugate operator $\hat{\psi}_{ \pm}$having anticommuting c-number eigenvalues. Suppose $\mid 00->$ is the normalized zero-eigenstate of $\hat{q}$ and $\hat{\psi}_{-}: \hat{q}\left|00->=0, \quad \hat{\psi}_{-}\right| 00->=0$. The state $\mid 00+>$ is defined by $\left|00+>=\hat{\psi}_{+}\right| 00->=0$, then $\hat{\psi}_{+}\left|00+>=0, \quad \hat{\psi}_{-}\right| 00+>=\mid 00->$. Taking into account that $\hat{\psi}_{ \pm}^{\dagger}=\hat{\psi}_{\mp}$, we get $<\mp 00\left|\hat{\psi}_{ \pm}=0, \quad<\mp 00\right| \hat{\psi}_{\mp}=< \pm 00 \mid$. Now we may introduce 
A new look at some aspects of geometry, particle physics, inertia, radiation and cosmology

the notation $\alpha, \beta, \ldots$ for the anticommuting eigenvalues of $\hat{\psi}_{ \pm}$. Consistency requires: $\alpha \hat{\psi}_{ \pm}=-\hat{\psi}_{ \pm} \alpha$, $\alpha|00 \pm>= \pm| 00 \pm>\alpha$. The eigenstates of $\hat{q}, \hat{\psi}_{-}$can be constructed as $\left|q \alpha->=e^{-i q \hat{p}-\alpha \hat{\psi}_{+}}\right| 00->$, and thus, $\hat{q}|q \alpha->=q| q \alpha->, \quad \hat{\psi}_{-}|q \alpha->=\alpha| q \alpha->$. Then, the $\hat{\pi}_{q}$ and $\hat{\psi}_{+}$eigenstates are obtained by Fourier transformation. Thereby the completeness relations hold: $-\int d \alpha d q\left|q \alpha \pm><\mp \alpha^{*} q\right|=1$, $-\int d \alpha \frac{d \pi_{q}}{2 \pi}\left|\pi_{q} \alpha \pm><\mp \alpha^{*} \pi_{q}\right|=1,-\int d \alpha d p\left|p \alpha \pm><\mp \alpha^{*} p\right|=1,-\int d \alpha \frac{d \pi_{p}}{2 \pi}\left|\pi_{p} \alpha \pm><\mp \alpha^{*} \pi_{p}\right|=1$. If $\mid t>$ is the sate of the system at time $t$, then the wave function is obtained by expanding this state with respect to the coordinate basis in q- and p-spaces: $\chi_{-}(q \alpha p \gamma t)=\psi_{-}(q \alpha t) \phi_{-}(p \gamma t)=<$ $+q \alpha^{*}\left|t><+p \gamma^{*}\right| t>$, and $\chi_{+}(q \beta p \delta t)=\psi_{+}(q \beta t) \phi_{+}(p \delta t)=<-q \beta^{*}\left|t><-p \delta^{*}\right| t>$, where $\psi_{+}(q \beta t)=$ $-\int d \alpha e^{-\alpha \beta} \psi_{-}(q \alpha t), \phi_{+}(p \delta t)=-\int d \gamma e^{-\gamma \delta} \phi_{-}(p \gamma t)$. On wave functions, the $\hat{\psi}_{ \pm}$are represented by anticommuting c-number operators: $\hat{\psi}_{-}=\zeta, \quad \hat{\psi}_{+}=\partial / \partial \zeta$, provided $\chi(q, p, \zeta)=\psi(q, \zeta) \phi(p, \zeta)=$ $\left[\psi_{1}(q)+\zeta \psi_{2}(q)\right]\left[\phi_{1}(p)+\zeta \phi_{2}(p)\right]$. These operators allow us consequently to define a pair of appropriate nilpotent supercharges $Q_{q+}=B_{q-} \otimes \hat{\psi}_{+}=\left(\begin{array}{cc}0 & B_{q-} \\ 0 & 0\end{array}\right), Q_{q-}=B_{q+} \otimes \hat{\psi}_{-}=\left(\begin{array}{cc}0 & 9 \\ B_{q+} & 0\end{array}\right)$, $Q_{p+}=B_{p-} \otimes \hat{\psi}_{+}=\left(\begin{array}{cc}0 & B_{p-} \\ 0 & 0\end{array}\right), Q_{p-}=B_{p+} \otimes \hat{\psi}_{-}=\left(\begin{array}{cc}0 & 0 \\ B_{p+} & 0\end{array}\right)$, which obey the required relations $\left\{Q_{ \pm}, Q_{ \pm}\right\}=0$. The operators $B_{ \pm}$can be presented as $B_{ \pm}=B_{1} \pm B_{2}$, where $B_{1}$ and $B_{2}$ are the hermitian operators. Accordingly, the operators $Q_{1}$ and $Q_{2}$ read $Q_{q 1}=Q_{q+}+Q_{q-}=B_{q 1} \sigma_{1}+B_{q 2} \sigma_{2}$, $Q_{q 2}=-i\left(Q_{q+}-Q_{q-}\right)=B_{q 1} \sigma_{2}-B_{q 2} \sigma_{1}$, and similar relations hold for the operators $Q_{p 1}$ and $Q_{q 2}$. It is easily verified that $Q_{q \pm}$ are the generators of SUSY transformations between $\hat{q}$ and $\hat{\psi}$, as well as $Q_{p \pm}$ are the generators of SUSY transformations between $\hat{p}$ and $\hat{\psi}:\left[Q_{q \pm}, \hat{q}\right]=-i \hat{\psi}_{ \pm}$, $\left[Q_{q \pm}, \hat{\pi}_{q}\right]=\mp W_{q}^{\prime}(\hat{q}) \hat{\psi}_{ \pm},\left\{Q_{q \pm}, \hat{\psi}_{\mp}\right\}=\hat{p}+\hat{\pi}_{q} \pm i W(\hat{q}),\left\{Q_{q \mp}, \hat{\psi}_{\mp}\right\}=0$, and $\left[Q_{p \pm}, \hat{p}\right]=-i \hat{\psi}_{ \pm}$, $\left[Q_{p \pm}, \hat{\pi}_{p}\right]=\mp V_{p}^{\prime}(\hat{p}) \hat{\psi}_{ \pm},\left\{Q_{p \pm}, \hat{\psi}_{\mp}\right\}=\hat{q}+\hat{\pi}_{p} \pm i V(\hat{p}),\left\{Q_{p \mp}, \hat{\psi}_{\mp}\right\}=0$. The SUSY Hamiltonians read $2 H_{q}=\left\{Q_{q 1}, Q_{q 2}\right\}=\left\{Q_{q+}, Q_{q-}\right\}=\left\{B_{q-}, B_{q+}\right\}+\left[B_{q-}, B_{q+}\right] \sigma_{3}, 2 H_{p}=\left\{Q_{p 1}, Q_{p 2}\right\}=\left\{Q_{p+}, Q_{p-}\right\}=$ $\left\{B_{p-}, B_{p+}\right\}+\left[B_{p-}, B_{p+}\right] \sigma_{3}$. Along the trajectories in $(p, q)$ space, however, this produces the extended Hamiltonian $H_{e x t}, H_{e x t}=\left(\begin{array}{cc}H_{+} & 0 \\ 0 & H_{-}\end{array}\right)=\left(\begin{array}{cc}H_{q+}-H_{p+} & 0 \\ 0 & H_{q-}-H_{p-}\end{array}\right)$, where $H_{q}=$ $\left(\begin{array}{cc}H_{q+} & 0 \\ 0 & H_{q-}\end{array}\right)=\frac{1}{2}\left(\begin{array}{cc}B_{q-} B_{q+} & 0 \\ 0 & B_{q+} B_{q-}\end{array}\right), H_{p}=\left(\begin{array}{cc}H_{p+} & 0 \\ 0 & H_{p-}\end{array}\right)=\frac{1}{2}\left(\begin{array}{cc}B_{p-} B_{p+} & 0 \\ 0 & B_{p+} B_{p-}\end{array}\right)$. Then $H_{\text {ext }}=\frac{1}{2}\left[\left(p+\pi_{q}\right)^{2}-\left(q+\pi_{p}\right)^{2}+W^{2}(q)-V^{2}(p)+\sigma_{3}\left(p+\pi_{q}\right) W(q)-i \sigma_{3}\left(q+\pi_{p}\right) V(p)\right]$.

From now on we replace $H_{\text {ext }}$ by $\bar{H}_{\text {ext }}$, and $\chi_{r}(q, p)$ by $\bar{\chi}_{r}(q, p)$, respectively, and retain former notational conventions. This realization characterizes two non-interacting point particles of equal mass $m=1$ moving along the real line under influence of the external scalar potential $U_{ \pm}=$ $U_{q \pm}-U_{p \pm} \equiv W^{2}(q)-V^{2}(p) \pm\left(W_{q}^{\prime}(q)-V_{p}^{\prime}(p)\right)$. The time evolution of the state $\mid t>$ is now given $\chi_{-}(q \alpha p \beta t)=-\int d \alpha^{\prime} d q^{\prime} d \beta^{\prime} d p^{\prime} K\left(q \alpha p \beta t \mid q^{\prime} \alpha^{\prime} p^{\prime} \beta^{\prime} t^{\prime}\right)$, provided by the kernel $K\left(q \alpha p \beta t \mid q^{\prime} \alpha^{\prime} p^{\prime} \beta^{\prime} t^{\prime}\right)=$ $<+q \alpha^{*} p \beta^{*}\left|e^{-i H_{e x t}\left(t-t^{\prime}\right)}\right| q^{\prime} \alpha^{\prime} p^{\prime} \beta^{\prime}>$, which can be evaluated by the path integral. Actually, an alternative approach to describe the state space and dynamics of the extended phase space quantum system is by the path integral, which reads $\mathcal{K}_{f f^{\prime}}\left(q p t \mid q^{\prime} p^{\prime} t^{\prime}\right)=<q p f\left|e^{-i H_{e x t}\left(t-t^{\prime}\right)}\right| q^{\prime} p^{\prime} f^{\prime}>$, where extended SUSY Hamiltonian can be represented as $H_{e x t}=\frac{1}{2}\left(\hat{\pi}_{q}^{2}+W^{2}(\hat{q})+i W_{q}^{\prime}(\hat{q})\left[\hat{\psi}_{1}, \hat{\psi}_{2}\right]\right)-\frac{1}{2}\left(\hat{\pi}_{p}^{2}+V^{2}(\hat{p})+\right.$ $\left.i V_{q}^{\prime}(\hat{p})\left[\hat{\psi}_{1}, \hat{\psi}_{2}\right]\right)$. To infer the extended Hamiltonian equivalently one may start from the c-number extended Lagrangian of extended phase space quantum field theory in $(0+1)$-dimensions in $q-$ and $p-$ spaces: $\mathcal{L}_{\text {ext }}(p, q, \dot{p}, \dot{q})=-\dot{q}_{i} p_{i}-q_{i} \dot{p}_{i}+\frac{1}{2}\left[\left(\frac{d q}{d t}\right)^{2}-W^{2}(q)\right]+f W_{q}^{\prime}(q)+\frac{1}{2}\left[\left(\frac{d p}{d t}\right)^{2}-V^{2}(p)\right]+f V_{p}^{\prime}(p)$. With the Hamiltonian $H_{\text {ext }}$, the path integral is diagonal: $\mathcal{K}_{f f^{\prime}}\left(q p t \mid q^{\prime} p^{\prime} t^{\prime}\right)=\mathcal{K}_{f f^{\prime}}\left(q t \mid q^{\prime} t^{\prime}\right) \mathcal{K}_{f f^{\prime}}\left(p t \mid p^{\prime} t^{\prime}\right)=$ $\delta_{f f^{\prime}} \int_{q^{\prime}}^{q} \mathcal{D} q \int_{p^{\prime}}^{p} \mathcal{D} p \exp \left(i \int_{t^{\prime}}^{t} \mathcal{L}_{\text {ext }}(p, q, \dot{p}, \dot{q}) d t\right)$. Knowing the path integral, it is sufficient to specify the initial wave function $\chi_{f}\left(q^{\prime}, p^{\prime}, t^{\prime}\right)$ to obtain all possible information about the system at any later time $t$, by $\chi_{f}(q, p, t)=$

$\sum_{f^{\prime}} \int d q^{\prime} d p^{\prime} \mathcal{K}_{f f^{\prime}}\left(q p t \mid q^{\prime} p^{\prime} t^{\prime}\right) \chi_{f^{\prime}}\left(q^{\prime}, p^{\prime}, t^{\prime}\right)$, The path integral becomes $\mathcal{K}\left(q \alpha p \beta t \mid q^{\prime} \alpha^{\prime} p^{\prime} \beta^{\prime} t^{\prime}\right)=$ $\int_{q^{\prime}, \alpha^{\prime}, p^{\prime}, \beta^{\prime}}^{q, \alpha, p} \mathcal{D} \mathcal{D} p \mathcal{D} \zeta \mathcal{D} \eta \exp \left(i \int_{t^{\prime}}^{t} \mathcal{L}_{\text {ext }}(p, q, \dot{p}, \dot{q}) d t\right)$. The functional integral is taken over all trajectories from $q^{\prime}, \alpha^{\prime}$ to $q, \alpha$ and $p^{\prime}, \beta^{\prime}$ to $p, \beta$ between the times $t^{\prime}$ and $t$. 


\subsection{The vacuum energy and the topology of superpotential}

The supersymmetry of quantum system is said to be a good symmetry (good SUSY) if the groundstate energy of $H_{\text {ext }}$ vanishes. In the other case, inf spec $H_{\text {ext }}>0$, SUSY is said to be broken. Let $\chi_{r}^{+}$and $\chi_{r}^{-}$denote the eigenstates of $H_{+}$and $H_{-}$, respectively, for the same positive eigenvalue $E_{r}>0: H_{ \pm} \chi_{r}^{ \pm}=E_{r} \chi_{r}^{ \pm}$. The SUSY transformation implies the relations $\chi_{r}^{+}=\frac{1}{\sqrt{E_{r}}} B_{+} \chi_{r}^{-}$, $\chi_{r}^{-}=\frac{1}{\sqrt{E_{r}}} B_{-} \chi_{r}^{+}$, where $B_{+} B_{-}=B_{q+} B_{q-}-B_{p+} B_{p-}, B_{-} B_{+}=B_{q-} B_{q+}-B_{p-} B_{p+}$. Note that under the replacement of SUSY potentials, $W \rightarrow-W$ and $V \rightarrow-V,\left(U_{ \pm} \rightarrow U_{\mp}\right)$, the roles of the two Hamiltonians $H_{+}$and $H_{-}$are interchanged. Hence, the sign of the SUSY potentials may be fixed by some convention. For good SUSY the groundsate $\chi_{0}$ of $H_{\text {ext }}$ either belongs to $H_{+}$or $H_{-}$ $H_{ \pm} \chi_{0}^{ \pm}=0 \Leftrightarrow B_{ \pm} \chi_{0}^{ \pm}=0$, or $\left(H_{q \pm}-H_{p \pm}\right) \chi_{0}^{ \pm}=0$. As far as $H_{q \pm}$ and $H_{p \pm}$ are independent, we have $H_{q \pm} \chi_{0}^{ \pm}=0, \quad H_{p \pm} \chi_{0}^{ \pm}=0$, or $B_{q \pm} \chi_{0}^{ \pm}=0, \quad B_{p \pm} \chi_{0}^{ \pm}=0$. Whence, $\left(\frac{d}{d q} \pm W(q)\right) \psi_{0}^{ \pm}(q)=0$, $\left(\frac{d}{d p} \pm V(p)\right) \phi_{0}^{ \pm}(p)=0, \chi_{0}^{ \pm}(q, p)>\equiv \psi_{0}^{ \pm}(q) \phi_{0}^{ \pm}(p)$, and $<q\left|\psi_{0}^{ \pm}>\equiv \psi_{0}^{ \pm}(q),<p\right| \phi_{0}^{ \pm}>\equiv \phi_{0}^{ \pm}(p)$. The functions $\psi_{0}^{ \pm}(q)$ and $\phi_{0}^{ \pm}(p)$ have to be square-integrable for SUSY to be a good symmetry. This requirement puts conditions on the SUSY potentials: $\int_{0}^{\infty} W\left(q^{\prime}\right) d q^{\prime} \rightarrow \infty$ at $q \rightarrow \pm \infty$ for $\psi_{0}^{+}$, $\int_{0}^{\infty} W\left(q^{\prime}\right) d q^{\prime} \rightarrow-\infty$ at $q \rightarrow \pm \infty$ for $\psi_{0}^{-}, \int_{0}^{\infty} V\left(p^{\prime}\right) d p^{\prime} \rightarrow \infty$ at $p \rightarrow \pm \infty$ for $\phi_{0}^{+}, \int_{0}^{\infty} V\left(p^{\prime}\right) d p^{\prime} \rightarrow-\infty$ at $p \rightarrow \pm \infty$ for $\phi_{0}^{-}$. Depending on the asymptotic behavior of the SUSY potentials one of the two functions $\chi_{0}^{ \pm}$will be normalizable (good SUSY) or both are not normalizable (broken SUSY). For continuous SUSY potentials $U_{ \pm}(p, q)$ the functions $W(q)$ and $V(p)$ must have an odd number of zeros (counted with their multiplicity) for SUSY to be good. A continuous SUSY potentials with an even number of zeros necessarily leads to a broken SUSY. Consequently, if $W(q)$ and $V(p)$ have a well-defined parity, and odd $W(q)$ and $V(p)$ lead to good SUSY, whereas an even $W(q)$ and $V(p)$ break SUSY: $W(-q)=-W(q) \Rightarrow U_{q \pm}(-q)=U_{q \pm}(q)$ (SUSY and parity are good in q-subspace), $W(-q)=W(q) \Rightarrow U_{q \pm}(-q) \neq U_{q \pm}(q)$ (SUSY and parity are broken in q-subspace), and correspondingly the similar conditions hold for $V(p)$ and $U_{q \pm}(p)$. The spectra of $H_{+}$and $H_{-}$are related as follows: $\operatorname{spec}\left(H_{-}\right) /\{0\}=\operatorname{spec}\left(H_{+}\right)(\operatorname{good} \operatorname{SUSY}), \operatorname{spec}\left(H_{-}\right)=\operatorname{spec}\left(H_{+}\right)(\operatorname{broken} \operatorname{SUSY})$.

Clearing up this situation the Witten index is turned out to be one of the useful tool which, according to the Atiyah-Singer index theorem associates with the operator index and depends only on the asymptotic values of SUSY potentials. This is a topological characteristic and does not vary with the variation of the parameters of theory. Thus, the Witten index reads $\Delta(\beta)=\operatorname{tr}\left(P e^{-\beta H_{e x t}}\right)=$ $\operatorname{tr}\left(P e^{-\beta\left(H_{q}-H_{p}\right)}\right), \quad \beta>0$. For a pure point spectrum of $H_{\text {ext }}$ this index is the difference of the number of spin-up states $(\uparrow)$ and spin-down states $(\downarrow)$ with zero energy: $\Delta(\beta)=N_{\uparrow}(E=0)-N_{\downarrow}(E=0)$. Note that the factor $e^{-\beta H_{e x t}}$ has only been introduced for regularization of the trace. The conditions of the positive-energy eigenstates cancel due to the pairwise degeneracy mentioned above. For a continuous spectrum this is not the case as the spectral densities for the spin-up and spin-down states are in general different due to which Witten index becomes $\beta$ dependent. Therefore, for simplicity reasons we assume purely discrete spectra. Then, $\Delta=\operatorname{ind} B=\operatorname{dim} \operatorname{ker} H_{-}-\operatorname{dim} \operatorname{ker} H_{+}=\operatorname{dim} \operatorname{ker} H_{q_{-}}+$ dim ker $H_{p+}-\operatorname{dim} \operatorname{ker} H_{q+}-\operatorname{dim} \operatorname{ker} H_{p-}$. Introducing a set of $H_{q}$ and $H_{p}$ upon reduction to the $q-$ or $p-$ space yields $\Delta=\frac{1}{2}[\operatorname{sgn} W(+\infty)+\operatorname{sgn} V(-\infty)-\operatorname{sgn} W(-\infty)-\operatorname{sgn} V(+\infty)]$. Hence for good SUSY one has $\Delta= \pm 1$ with the ground state belonging to $H_{ \pm}$. For broken SUSY one has $\Delta=0$. Although even small non-zero energy expectation value $\varepsilon=\left(\chi_{1}, H_{\text {ext }} \chi_{1}\right)$ gives direct evidence for the SUSY breaking in the extended phase space quantum mechanical system, a more practical measure for the SUSY breaking, in particular, in field theories is the expectation value of an auxiliary field, which can be replaced by its equation of motion right from the start: $\langle F\rangle=\left(\chi_{\uparrow}, i\left\{Q_{+}, \sigma_{-}\right\} \chi_{\uparrow}\right)$, where as we mentioned above the solutions for non-zero energy come in pairs of the form $\chi_{\uparrow}(q, p)$ or $\chi_{\downarrow}(q, p)$ related by supersymmetry. Taking into account the relation $Q_{+} \chi_{\uparrow}=0$, where $Q_{+}$commutes with $H_{\text {ext }}$ which means that the intermediate state must have the same energy as $\chi$, the can be re-written in terms of a complete set of states: $<F>=i\left(\chi_{\uparrow}, Q_{+} \chi_{\downarrow}\right)\left(\chi_{\downarrow}, \hat{\psi}_{-}, \chi_{\uparrow}\right)$. We have $\varepsilon=<$ $H_{\text {ext }}>=\frac{1}{2}\left(\chi_{\uparrow}, Q_{+} \chi_{\downarrow}\right)\left(\chi_{\downarrow}, Q_{-} \chi_{\uparrow}\right)=\varepsilon_{q}-\varepsilon_{p}=<H_{q}>-<H_{p}>=\left(\psi_{\uparrow}, H_{q} \psi_{\uparrow}\right)-\left(\phi_{\uparrow}, H_{p} \phi_{\uparrow}\right)=$ $\frac{1}{2}\left(\psi_{\uparrow}, Q_{q+} \psi_{\downarrow}\right)\left(\psi_{\downarrow}, Q_{q-} \psi_{\uparrow}\right)-\frac{1}{2}\left(\phi_{\uparrow}, Q_{p+} \phi_{\downarrow}\right)\left(\phi_{\downarrow}, Q_{p-} \phi_{\uparrow}\right)$. 


\subsection{A shape-invariance of exactly solvable SUSY potentials}

SUSY quantum mechanics provides an elegant and useful prescripton for obtaining closed analytic expressions both for the energy eigenvalues and eigenstates of a large class of Schrödinger-like equations. The key point in this are the SUSY partner Hamiltonians $H_{-}=B_{-} B_{+}$and $H_{+}=B_{+} B_{-}$. The operators in this factorization are expressed in terms of the SUSY potentials. The potentials for which the Schrödinger-like equations are exactly solvable share an integrability conditions called shape-invariance. The concept of shape-invariance in SUSY quantum mechanics has proven to be very useful because it leads immediately to exactly solvable potentials. An extended Hamiltonian $H_{\text {ext }}$ of SUSY quantum mechanics can be treated as a set of two ordinary two-dimensional partner Hamiltonians $H_{ \pm}=\frac{1}{2}\left[\pi_{q}^{2}-\pi_{p}^{2}+U_{ \pm}(q, p)\right]$. Due to SUSY they have the same energy spactra at arbitrary functions $W(q)$ and $V(p)$, except the groundstate of $H_{-}$(defined in accordance with usual convention) which has no corresponding state in the spectra of $H_{+}$. The partner potentials $U_{ \pm}(q, p)$ are called shapeinvariant if they satisfy an integrability condition $U_{ \pm}(a, q, p)=U_{-}\left(a_{1}, q, p\right)+R\left(a_{1}\right), a_{1}=f(a)$, where $a$ and $a_{1}$ are a set of parameters that specify phase-space-independent properties of the potentials, and the reminder $R\left(a_{1}\right)$ is independent of $(q, p)$. Although this looks like a satisfactory state of affairs, we may not always be so fortunate to have such potentials at our disposal. In fact a shape-invariance is not the most general integrability condition as not all exactly solvable potentials seem to be shapeinvariant. Using the standard technique, we construct a series of Hamiltonians $H_{n}, n=0,1,2, \ldots$, $H_{n}=\frac{1}{2}\left[\pi_{q}^{2}-\pi_{p}^{2}+U_{-}\left(a_{n}, q, p\right)+\S_{k=1}^{n} R\left(a_{k}\right)\right]$, where $a_{n}=f^{(n)}(a)$ ( $n$ means the number of multiple applications.) Comparing the spectra $H_{n}$ and $H_{n+1}$, we obtain $H_{n+1}=\frac{1}{2}\left[\pi_{q}^{2}-\pi_{p}^{2}+U_{+}\left(a_{n}, q, p\right)+\S_{k=1}^{n} R\left(a_{k}\right)\right]$. We see that the Hamiltonians $H_{n}$ and $H_{n+1}$ have the same energy spectra, except the groundstate of $H_{n}$, the energy of which is equal $\S_{k=1}^{n} R\left(a_{k}\right)$. Going through $H_{n}$ to $H_{n-1}$ and so on, we subsequently obtain the initial Hamiltonian $H_{0}=H_{-}=\frac{1}{2}\left[\pi_{q}^{2}-\pi_{p}^{2}+U_{-}(a, q, p)\right]$, the groundstate of which is equal zero, but all the other energy levels coincide with the lower levels of Hamiltonians $H_{n}$. Continuing along this line, the entire energy spectrum of $H_{\text {ext }}$ reads $\widetilde{E}_{n}=\S_{k=1}^{n} R\left(a_{k}\right)$. Hence the spectrum of Hamiltonian with the potential $U(a, q, p)=U_{-}(a, q, p)+C(a)$ has the form $E_{n}=\widetilde{E}_{n}+C(a)=\S_{k=1}^{n} R\left(a_{k}\right)+C(a)$. Instead of developing the full machinery here, we will illustrate this in passing in the following example.

Example: Scarf potential. To demonstrate practical merits of shape-invariance of exactly solvable extended SUSY potentials, we now obtain analytic expressions for the entire energy spectrum of onedimensional problem with extended Scarf potential without ever referring to underlying differential equation. This potential $U(a, b, q, p)=U_{q}(a, b)-U_{p}(b, p)=-\frac{a(a+1)}{2 c h^{2} q}+\frac{b(b+1)}{2 c h^{2} p}$, will be of vital interest for the theory of solitons in extended phase space formalism. In case at hand we have $W(q)=a$ th $q$ and $V(p)=b$ th $p$, hence $U_{ \pm}(a, b, q, p)=U_{q \pm}(a, q)-U_{p \pm}(b, p)=-\frac{a(a \mp 1)}{2 c h^{2} q}+\frac{a^{2}}{2}+\frac{b(b \pm 1)}{2 c h^{2} p}-\frac{b^{2}}{2}$. This yields $a_{1}=f_{1}(a)=a-1 ; a_{n}=a-n ; C_{1}(a)=-\frac{a^{2}}{2}, b_{1}=f_{2}(b)=b-1 ; b_{n}=b-n ; C_{2}(b)=-\frac{b^{2}}{2}, \sum_{k=1}^{n} R_{1}\left(a_{k}\right)=$ $\frac{a^{2}-a_{n}^{2}}{2}, \sum_{k=1}^{n} R_{2}\left(b_{k}\right)=\frac{b^{2}-b_{n}^{2}}{2}$. The entire energy spectrum of the $H_{\text {ext }}$ can be easily obtained as $E_{n}=E_{q n}-E_{p n}=-\frac{a_{n}^{2}}{2}+\frac{b_{n}^{2}}{2}=-\frac{(a-n)^{2}}{2}+\frac{(b-n)^{2}}{2}$. One final observation is worth recording. The shape-invariance has underlying algebraic structure of Lie algebras, which transform the parameters of the potentials. Shape-invariance algebra in general is an infinite-dimensional. However, under some conditions they become finite-dimensional. The Hamiltonian of exactly solvable systems can be written as a linear or quadratic function of an underlying algebra, and all the quantum states of these systems can be determined by independent group theoretical methods with a general change of parameters which involves nonlinear extensions of Lie algebras.

\section{Classical analog of extended phase space SUSY and its breaking}

It is certainly desirable to derive the classical analog of the extended phase space quantum mechanics of the particle with odd degrees of freedom directly from what may be taken as the first principle (?). Let us consider a nonrelativistic particle of unit mass with two $(\alpha=1,2)$ odd (Grassmann) degrees of freedom. The classical extended Lagrangian can be written $\mathcal{L}_{\text {ext }}(p, q, \dot{p}, \dot{q})=$ $-\dot{q} p-q \dot{p}+\frac{1}{2} \dot{q}^{2}-F(q)+\frac{1}{2} \dot{p}^{2}-G(p)-R(q, p) N+\frac{1}{2} \psi_{\alpha} \dot{\psi}_{\alpha}$, provided by $N=\psi_{1} \psi_{2}=-i \psi_{+} \psi_{-}$. Here $F(q): \mathcal{R} \rightarrow \mathcal{R}, \quad G(p): \mathcal{R} \rightarrow \mathcal{R}$ and $R(q, p): \mathcal{R} \rightarrow \mathcal{R}$ are arbitrary piecewise continuously differentiable functions given over the 1-dimensional Euclidean space $\mathcal{R}$. The $\psi_{\alpha}$ are two odd (Grassmann) 
degrees of freedom. The nontrivial Poisson-Dirac brackets of the system are $\left\{q, \pi_{q}\right\}=1, \quad\left\{p, \pi_{p}\right\}=$ $1, \quad\left\{\psi_{\alpha}, \psi_{\beta}\right\}=\delta_{\alpha \beta},\left\{\psi_{+}, \psi_{-}\right\}=1, \quad \psi_{ \pm}^{2}=0, \psi_{ \pm}=\frac{1}{\sqrt{2}}\left(\psi_{1} \pm i \psi_{2}\right)$. The extended Hamiltonian $H_{\text {ext }}$ reads $H_{\text {ext }}\left(p, q, \pi_{p}, \pi_{q}\right)=\frac{1}{2}\left(p+\pi_{q}\right)^{2}+F^{2}(q)-\frac{1}{2}\left(q+\pi_{p}\right)^{2}-G^{2}(p)+R(q, p) N$, which reduces to $H_{\text {ext }}\left(p, q, \pi_{p}, \pi_{q}\right)=\frac{1}{2} \pi_{q}^{2}+F^{2}(q)-\frac{1}{2} \pi_{p}^{2}-G^{2}(p)+R(q, p) N$. This Hamiltonian yields the following equations of motion: $\dot{q}=\pi_{q}, \quad \dot{p}=\pi_{p}, \dot{\pi}_{q}=-F_{q}^{\prime}(q)-R_{q}^{\prime}(q, p) N, \dot{\pi}_{p}=-G_{p}^{\prime}(p)+R_{p}^{\prime}(q, p) N$, $\dot{\psi}_{ \pm}= \pm i R(q, p) \psi_{ \pm}$. A prime will indicate differentiation with respect either to $q$ or $p$. Thus, $N$ is the integral of motion additional to $H_{\text {ext }}$. Along the trajectories $q(t)$ and $p(t)$ in $(p, q)-$ spaces, the solution to equations of motion for odd variables is $\psi_{ \pm}(t)=\psi_{ \pm}\left(t_{0}\right) \exp \left[ \pm i \int_{t_{0}}^{t} R(q(\tau), p(\tau)) d \tau\right]$. Hence the odd quantities $\theta_{ \pm}=\theta_{ \pm}(t) \exp \left[\mp i \int_{t_{0}}^{t} R(q(\tau), p(\tau)) d \tau\right]$ are nonlocal in time integrals of motion. In trivial case $R=0$, we have $\dot{\psi}_{ \pm}=0$, and $\theta_{ \pm}=\theta_{ \pm}$. Suppose the system has even complex conjugate quantities $B_{q, p \pm}, \quad\left(B_{q, p+}\right)^{*}=B_{q, p-}$, whose evolution looks up to the term proportional to $N$ like the evolution of odd variables. Then local odd integrals of motion could be constructed in the form $Q_{q, p \pm}=B_{q, p \mp} \psi_{ \pm}$. Let us introduce the oscilliator-like bosonic variables $B_{q, p \pm}$ in $q-$ and $p$-representations $B_{q \mp}: \mathcal{L}^{2}(\mathcal{R}) \rightarrow \mathcal{L}^{2}(\mathcal{R}), \quad B_{q \mp}=\left[p+\pi_{q} \pm i W(q)\right], B_{p \mp}: \mathcal{L}^{2}(\mathcal{R}) \rightarrow \mathcal{L}^{2}(\mathcal{R}), \quad B_{p \mp}=$ $\left[q+\pi_{p} \pm i V(p)\right]$. The $W(q): \mathcal{R} \rightarrow \mathcal{R}$ and $V(p): \mathcal{R} \rightarrow \mathcal{R}$ are the piecewise continuously differentiable functions called SUSY potentials. In particular case if $R(q, p)=R_{q}(q)-R_{p}(p)$, for the evolution of $B_{q, p \pm}$ we obtain $\dot{B}_{q \mp}=\left[-\left(F_{q}^{\prime}+R_{q}^{\prime} N\right) \pm i W_{q}^{\prime}(q)\left(p+\pi_{q}\right)\right], \quad \dot{B}_{p \mp}=\left[-\left(G_{p}^{\prime}+R_{p}^{\prime} N\right) \pm i V_{p}^{\prime}(p)\left(q+\pi_{p}\right)\right]$. Consequently, $\dot{Q}_{q \pm}= \pm i\left[\left(W_{q}^{\prime}-R_{q \pm}^{\prime}\right) \pm i\left(F_{q}^{\prime}-W W_{q}^{\prime}\right) \psi_{\mp}\right], \dot{Q}_{p \pm}= \pm i\left[\left(V_{p}^{\prime}-R_{p \pm}^{\prime}\right) \pm i\left(G_{p}^{\prime}-V V_{p}^{\prime}\right) \psi_{\mp}\right]$. This shows that either $\dot{Q}_{q \pm}=0$ or $\dot{Q}_{p \pm}=0$ when $W_{q}^{\prime}(q)=R_{q \pm}^{\prime}(q)$ and $F_{q}^{\prime}=\frac{1}{2}\left(W^{2}\right)_{q}^{\prime}$ or $V_{p}^{\prime}(p)=R_{p \pm}^{\prime}(p)$ and $G_{p}^{\prime}=\frac{1}{2}\left(V^{2}\right)_{p}^{\prime}$, respectively. Therefore, when the functions $R_{q, p}$ and $F(q), G(p)$ are related as $R_{q \pm}^{\prime}(q)=W_{q}^{\prime}(q), \quad F_{q}=\frac{1}{2}\left(W^{2}\right)+C_{q}, R_{p \pm}^{\prime}(p)=V_{p}^{\prime}(p), \quad F_{p}=\frac{1}{2}\left(V^{2}\right)+C_{p}$, where $C_{q, p}$ are constants, then odd quantities $Q_{q, p \pm}$ are integrals of motion in addition to $H_{\text {ext }}$ and $N$. Let us present $H_{\text {ext }}$ in the form $H_{\text {ext }}=H_{q}-H_{p}$, where $H_{q}=\frac{1}{2} \pi_{q}^{2}+F^{2}(q)+R_{q} N, \quad H_{p}=\frac{1}{2} \pi_{p}^{2}+G^{2}(p)+R_{p} N$. Then, $Q_{q, p \pm}$ and $N$ together with the $H_{q}$ and $H_{p}$ form the classical analog of the extended phase space SUSY algebra $\left\{Q_{q, p+}, Q_{q, p-}\right\}=-i\left(H_{q, p}-C_{q, p}\right), \quad\left\{H_{q, p}, Q_{q, p \pm}\right\}=\left\{Q_{q, p \pm}, Q_{q, p \pm}\right\}=0,\left\{N, Q_{q, p \pm}\right\}=$ $\pm i Q_{q, p \pm}, \quad\left\{N, H_{q, p}\right\}=0$, with constants $C_{q, p}$ playing a role of a central charges in $(q, p)$ - spaces, $N$ is classical analog of the grading operator. Putting $C_{q}=C_{p}=0$, we arrive at the classical analog of the extended phase space SUSY quantum mechanics given by the extended Lagrangian $\mathcal{L}_{\text {ext }}(p, q, \dot{p}, \dot{q})=\frac{1}{2} \pi_{q}^{2}-\frac{1}{2} W^{2}(q)+\frac{1}{2} \pi_{p}^{2}-\frac{1}{2} V^{2}(p)+\psi_{1} \psi_{2}\left(W_{q}^{\prime}+V_{p}^{\prime}\right)+\frac{1}{2} \psi_{\alpha} \dot{\psi}_{\alpha}$. We conclude that the classical system is characterized by the presence of two additional local in time odd integrals of motion being supersymmetry generators. Along the actual trajectories in $q$-space, the lagrangian reproduces the results obtained in previous section.

\subsection{Solution of the extended Schrödinger equation with small energy eigenvalue}

First, we use the iterative scheme to find the approximate groundstate solutions to the extended Schrödinger-like equation $H_{\text {ext }} \chi(q, p)=\left(H_{q}-H_{p}\right) \chi(q, p)=\varepsilon \chi(q, p)$, with energy $\varepsilon$. We will then use these solutions to calculate the parameters which measure the breaking of extended SUSY such as the groundstate energy. The approximation, which went into the derivation of solutions meets our interest that the graoundstate energy $\varepsilon$ is supposedly small. As we mentioned above the solutions for non-zero $\varepsilon$ come in pairs of the form $\chi_{\uparrow}(q, p)=\left(\begin{array}{c}\chi_{1}(q, p) \\ 0\end{array}\right)$ or $\quad \chi_{\downarrow}(q, p)=\left(\begin{array}{c}0 \\ \chi_{2}(q, p)\end{array}\right)$, related by supersymmetry, where $\chi_{1,2}(q, p)=\psi_{1,2}(q) \phi_{1,2}(p)$. The state space of the system is defined by all the normalizable solutions and the individual states are characterized by the energies $\varepsilon_{q}$ and $\varepsilon_{q}$ and the fermionic quantum number $f$. One of these solutions is acceptable only if $W(q)$ and $V(p)$ become infinite at both $q \rightarrow \pm \infty$ and $p \rightarrow \pm \infty$, respectively, with the same sign. If this condition is not satisfied, neither of the solutions is normalizable, and they cannot represent the groundstate of the system. The following relations between energy eigenstates with fermionic quantum number $\pm \frac{1}{2}$ hold: $\left[\left(\frac{\partial}{\partial q}+W_{q}(q)\right)-\left(\frac{\partial}{\partial p}+V_{p}(p)\right)\right] \psi_{1}(q) \phi_{1}(p)=\sqrt{2 \varepsilon_{q}} \psi_{2}(q) \phi_{1}(p)-\sqrt{2 \varepsilon_{p}} \psi_{1}(q) \phi_{2}(p)$, and $\left[\left(-\frac{\partial}{\partial q}+W_{q}(q)\right)-\left(-\frac{\partial}{\partial p}+V_{p}(p)\right)\right] \psi_{2}(q) \phi_{2}(p)=\sqrt{2 \varepsilon_{q}} \psi_{1}(q) \phi_{2}(p)-\sqrt{2 \varepsilon_{p}} \psi_{2}(q) \phi_{1}(p)$, where $\varepsilon=\varepsilon_{q}-\varepsilon_{p}$, $\varepsilon_{q}$ and $\varepsilon_{p}$ are the eigenvalues of $H_{q}$ and $H_{p}$, respectively. The technique now is to devise an iterative approximation scheme by taking a trial wave function for $\chi_{2}(q, p)$, substitute this into the first equation 
and integrate it to obtain an approximation for $\chi_{1}(q, p)$. This can be used as an ansatz in the second equation to find an improved solution for $\chi_{2}(q, p)$, etc. The procedure converges for well-behaved potentials with a judicious choice of initial trial function. If the $W_{q}$ and $V_{p}$ are odd, then $\psi_{1}(-q)=$ $\psi_{2}(q), \phi_{1}(-p)=\phi_{2}(p)$, since they satisfy the same eigenvalue equation. The independent nature of $q$ and $p$ gives the freedom of taking $q=0, \quad p=0$ which yield an expression for energies: $\sqrt{2 \varepsilon_{q}}=$ $W(0)+\psi_{1}^{\prime}(0) / \psi_{1}(0), \sqrt{2 \varepsilon_{p}}=V(0)+\phi_{1}^{\prime}(0) / \phi_{1}(0)$. Suppose the potentials $W_{q}(q)$ and $V_{p}(p)$ have a maximum, at $q_{-}$and $p_{-}$, and minimum, at $q_{+}$and $p_{+}$, respectively. For the simplicity sake we choose the trial wave functions as $\psi_{1,2}^{(0)}(q)=\delta\left(q-q_{ \pm}\right), \phi_{1,2}^{(0)}(p)=\delta\left(p-p_{ \pm}\right)$. The normalization constant $N^{\prime}$ is $N^{\prime}=\left(\int_{q_{-}}^{\infty} d q e^{-2}{ }_{0}^{\grave{q}} W\left(q^{\prime}\right) d q^{\prime} \int_{p_{-}}^{\infty} d p e^{-2} \underset{0}{\stackrel{p^{\prime}}{\prime}} V\left(p^{\prime}\right) d p^{\prime}\right)^{3 / 2}$. The energy expectation value $\varepsilon=\left(\chi_{1}, H_{\text {ext }} \chi_{1}\right)$ gives the same result as that obtained for odd potentials. Assuming the exponentials $e^{-2}{ }_{q_{-}}^{\dagger} W(q) d q$ and $e^{-2}{ }_{p_{-}}^{t} V(p) d p$ to be small, which is correct to the same approximations, the difference is negligible and the integrals in both cases may be replaced by gaussians around $q_{+}$and $p_{+}$, respectively. Hence, it is straightforward to obtain $\varepsilon=\frac{\hbar W^{\prime}\left(q_{+}\right)}{2 \pi} e^{-2 \Delta W / \hbar}-\frac{\hbar V^{\prime}\left(p_{+}\right)}{2 \pi} e^{-2 \Delta V / \hbar}$, which gives direct evidence for the SUSY breaking in the extended phase space quantum mechanical system. Here we have reinstated $\hbar$, to show the order of adopted approximation, and its non-perturbative nature. We also denoted $\Delta W=\int_{q_{+}}^{q_{-}} W(q) d q, \quad \Delta V=\int_{p_{+}}^{p_{-}} V(p) d p$. However, a more practical measure for the SUSY breaking, in particular, in field theories is the expectation value of an auxiliary field, which can be replaced by its equation of motion right from the start: $\langle F\rangle=\left(\chi_{\uparrow}, i\left\{Q_{+}, \sigma_{-}\right\} \chi_{\uparrow}\right)$, etc. Hence we obtain $\left(\chi_{\uparrow}, Q_{+} \chi_{\downarrow}\right)\left(\chi_{\downarrow}, \hat{\psi}_{-} \chi_{\uparrow}\right)=2 i \sqrt{\varepsilon}\left[\left(\frac{\varepsilon_{q}}{\varepsilon}\right) \sqrt{\varepsilon_{q}} \sqrt{\frac{W^{\prime}\left(q_{-}\right)}{\pi}} \Delta q-\left(\frac{\varepsilon_{p}}{\varepsilon}\right) \sqrt{\varepsilon_{p}} \sqrt{\frac{V^{\prime}\left(p_{-}\right)}{\pi}} \Delta p\right]$, and that $<F>=-2 \sqrt{\varepsilon}\left[\left(\frac{\varepsilon_{q}}{\varepsilon}\right) \sqrt{\frac{W^{\prime}(q+)}{\pi}} e^{-2 \Delta W} \Delta q-\left(\frac{\varepsilon_{p}}{\varepsilon}\right) \sqrt{\frac{V^{\prime}(p)}{\pi}} e^{-2 \Delta V} \Delta p\right]$.

\subsection{An extended SUSY breaking in the instanton picture}

The matrix elements of $\hat{\psi}_{ \pm}, Q_{q \pm}$ and $Q_{p \pm}$ can be calculated in the background of the classical solution $\dot{q}_{c}=-W_{c}$ and $\dot{p}_{c}=-V_{c}$. In doing this we re-write the matrix element in terms of eigenstates of the conjugate operator $\hat{\psi}_{ \pm}$. In the limit $T \rightarrow-i \infty$, this reduces to $\sqrt{\varepsilon}<+0 q_{+} p_{+}\left|Q_{+}\right| q_{-} p_{-} 0-><$ $-0 q_{-} p_{-}\left|\hat{\psi}_{-}\right| q_{+} p_{+} 0+>=\sqrt{\varepsilon_{q}}<+0 q_{+}\left|Q_{q+}\right| q_{-} 0-><-0 q_{-}\left|\hat{\psi}_{-}\right| q_{+} 0+>-\sqrt{\varepsilon_{p}}<+0 p_{+}\left|Q_{p+}\right| p_{-} 0-><$ $-0 p_{-}\left|\hat{\psi}_{-}\right| p_{+} 0+>$, which, in turn, can be presented by path integrals defined in terms of anticommuting c-number operators $\zeta$ and $\eta$ with Euclidean actions of the instantons in $q-$ and $p$ - spaces, respectively. These functional integrals include an integration over instanton time $\tau_{0}$ which is due to the problem of zero modes of the bilinear terms in Euclidean actions. This arises from timetransformation of instantons, and SUSY transformations on them, respectively. The existence of zero modes gives rise to non-gaussian behaviour of the functional integral. Due to it the matrix elements above do not receive any contributions from either no-instanton or anti-instanton configurations. The zero mode problem is solved by introducing a collective coordinate $\tau_{0}$ replacing the bosonic zero mode. Whereas, the funcional integrals depend only on the difference $\tau-\tau_{0}$. Note also that multi-instanton configurations could contribute in principle, provided they have not more than one normalizable fermionic zero mode. But, their contribution is clearly smaller with respect to $\sqrt{2 \varepsilon_{q}}$ and $\sqrt{2 \varepsilon_{p}}$. In the case when the SUSY potentials in $q-$ and $p$ - spaces have more than two extrema $q_{\nu}$ and $p_{\mu}, \nu, \mu=1,2, \ldots, N$, one can put conditions on the SUSY potentials $\int_{0}^{\infty} W\left(q^{\prime}\right) d q^{\prime} \rightarrow \infty$ at $q \rightarrow \pm \infty$ for $\psi_{0}^{+}, \int_{0}^{\infty} W\left(q^{\prime}\right) d q^{\prime} \rightarrow-\infty$ at $q \rightarrow \pm \infty$ for $\psi_{0}^{-}$, and similar for $V(p)$, that the extrema are well separated: $\int_{q_{\nu}}^{q_{\nu}+1} W\left(q^{\prime}\right) d q^{\prime} \gg 1$, and $\int_{p_{\mu}}^{q_{\mu}+1} V\left(p^{\prime}\right) d p^{\prime} \gg 1$. Around each of the classical minima $q_{\nu}$ and $p_{\mu}$ of the potentials $W^{2}(q)$ and $V^{2}(p)$, respectively, one can approximate the theory by a suppersymmetric harmonic oscilliator. Then there are $N$ ground states which have zero energy. These states are described by upper or lower component of the wave function, depending on whether $\nu$ and $\mu$ are odd or even. With this provision the functional integrals can be calculated, which allow us consequently to write down $<+0 q_{+}\left|Q_{q+}\right| q_{-} 0->=i \sqrt{\frac{W_{c}^{\prime}\left(q_{+}\right)}{\pi}} e^{-\Delta W_{c}},<-0 q_{-}\left|\hat{\psi}_{-}\right| q_{+} 0+>=$ $\sqrt{\frac{W_{c}^{\prime}\left(q_{+}\right)}{\pi}} e^{-\Delta W_{c}} \Delta q_{c}$, etc. Then, we arrive at the $<+0 q_{+} p_{+}\left|Q_{+}\right| q_{-} p_{-} 0-><-0 q_{-} p_{-}\left|\hat{\psi}_{-}\right| q_{+} p_{+} 0+>=$ $2 i \sqrt{\varepsilon}\left[\left(\frac{\varepsilon_{q}}{\varepsilon}\right) \sqrt{\varepsilon_{q}} \sqrt{\frac{W_{c}^{\prime}\left(q_{+}\right)}{\pi}} \Delta q_{c}-\left(\frac{\varepsilon_{p}}{\varepsilon}\right) \sqrt{\varepsilon_{p}} \sqrt{\frac{V_{c}^{\prime}\left(p_{+}\right)}{\pi}} \Delta p_{c}\right]$, and, as its inevitable corollary, to final solution. 
This proves that the extended SUSY breaking has resulted from tunnelling between the classical vacua of the theory. The corrections to this picture are due to higher order terms and quantum tunneling effects.

\section{Operator manifold approach to geometry and particle physics}

The operator manifold formalism enables the unification of the geometry and the field theory. It yields the quantization of geometry drastically different from earlier suggested schemes. We explored the query how did the geometry and fields, as they are, come into being. The substance out of which the geometry and fields are made is the 'primordial structures'. The mathematical framework of the OM formalism reveals the fundamental concepts of the particle physics. The primordial structures are designed to posses certain physical properties. The processes of their creation and annihilation in the lowest state (the regular structures) just are described by the OM formalism. In all the higher states the primordial structures are distorted ones, namely they have undergone the distortion transformations. The distortion transformation functions are the operators acting in the space of the internal degrees of freedom (colours) and imply the fundamental Incompatibility relations, which hold for both the local and the global distortion rotations. They underly the most important symmetries such as the internal symmetries $U(1), S U(2), S U(3)$, the $S U(2) \otimes U(1)$ symmetry of electroweak interactions, etc. Our major purpose is to prove the idea that the Geometry and Fields, with the Internal symmetries and all interactions, as well the four major principles of Relativity (Special and General), Quantum, Gauge and Colour confinement are derivative, and they come into being simultaneously. For illustrative purposes below we briefly consider just only a few preliminary examples from the two important aspects of mathematical background of the OM-formalism (Ter-Kazarian, 1884, 1996, 1999a). Thereby we suppress the indices without notice.

\subsection{Quantization of Geometry}

Unifying the geometry and particles into one framework the OM formalism is analogous to the method of secondary quantization with appropriate expansion over the geometric objects. We proceed at once to the secondary quantization of geometry by substituting the basis elements for the creation and annihilation operators acting in the configuration space of occupation numbers. Instead of pseudo vectors $O_{\lambda}$ we introduce the operators supplied by additional index $(r)$ referring to the quantum numbers of corresponding state $\hat{O}_{1}^{r}=O_{1}^{r} \alpha_{1}, \hat{O}_{2}^{r}=O_{2}^{r} \alpha_{2}, \hat{O}_{r}^{\lambda}={ }^{*} \delta^{\lambda \mu} \hat{O}_{\mu}^{r}=\left(\hat{O}_{\lambda}^{r}\right)^{+},\left\{\hat{O}_{\lambda}^{r}, \hat{O}_{\tau}^{r^{\prime}}\right\}=$ $\delta_{r r^{*}}{ }^{*} \delta_{\lambda \tau} I_{2}$. The matrices $\alpha_{\lambda}$ satisfy the condition $\left\{\alpha_{\lambda}, \alpha_{\tau}\right\}={ }^{*} \delta_{\lambda \tau} I_{2}$, where $\alpha^{\lambda}={ }^{*} \delta^{\lambda \mu} \alpha_{\mu}=\left(\alpha_{\lambda}\right)^{+}$, and $I_{2}=\left(\begin{array}{ll}1 & 0 \\ 0 & 1\end{array}\right)$. For example $\alpha_{1}=\left(\begin{array}{cc}0 & 1 \\ 0 & 0\end{array}\right), \alpha_{2}=\left(\begin{array}{cc}0 & 0 \\ 1 & 0\end{array}\right)$. The creation $\hat{O}_{1}^{r}$ and annihilation $\hat{O}_{2}^{r}$ operators are acting as follows: $\hat{O}_{1}^{r}\left|0>=O_{1}^{r}\right| 1>, \hat{O}_{2}^{r}\left|1>=O_{2}^{r}\right| 0>$, where $|0>\equiv| 0,0, \ldots>$ and $|1>\equiv| 0, \ldots, 1, \ldots>$ are respectively the nonoccupied vacuum state and the one occupied state. Thus, $\hat{O}_{1}^{r}\left|1>=0, \hat{O}_{2}^{r}\right| 0>=0$. A matrix realization of such states, for instance, can be: $\mid 0>\equiv \chi_{1}=\left(\begin{array}{c}0 \\ 1\end{array}\right)$, $\mid 1>\equiv \chi_{2}=\left(\begin{array}{l}1 \\ 0\end{array}\right)$. Hence $\chi_{0} \equiv \mid 0>=\prod_{r=1}^{N}\left(\chi_{1}\right)_{r}$ and $\chi_{r^{\prime}} \equiv \mid 1>=\left(\chi_{2}\right)_{r^{\prime}} \prod_{r \neq r^{\prime}}\left(\chi_{1}\right)_{r}$. Also, instead of ordinary basis vectors we introduce the operators $\hat{\sigma}_{\alpha}^{r} \equiv \delta_{\alpha \beta \gamma} \sigma_{\beta}^{r} \widetilde{\sigma}_{\gamma}$, where $\widetilde{\sigma}_{\gamma}$ are Pauli's matrices such that $<\sigma_{\alpha}^{r}, \sigma_{\beta}^{r^{\prime}}>=\delta_{r r^{\prime}} \delta_{\alpha \beta}, \hat{\sigma}_{r}^{\alpha}=\delta^{\alpha \beta} \hat{\sigma}_{\beta}^{r}=\left(\hat{\sigma}_{\alpha}^{r}\right)^{+}=\hat{\sigma}_{\alpha}^{r},\left\{\hat{\sigma}_{\alpha}^{r}, \hat{\sigma}_{\beta}^{r^{\prime}}\right\}=2 \delta_{r r^{\prime}} \delta_{\alpha \beta} I_{2}$. The vacuum state $\mid 0>\equiv \varphi_{1(\alpha)}$ and the one occupied state $\mid 1_{(\alpha)}>\equiv \varphi_{2(\alpha)} \operatorname{read}: \varphi_{1(\alpha)} \equiv \chi_{1}, \varphi_{2(1)}=\left(\begin{array}{c}1 \\ 0\end{array}\right), \varphi_{2(2)}=\left(\begin{array}{c}-i \\ 0\end{array}\right)$, $\varphi_{2(3)}=\left(\begin{array}{c}0 \\ -1\end{array}\right)$, thus, $\hat{\sigma}_{\alpha}^{r} \varphi_{1(\alpha)}=\sigma_{\alpha}^{r} \varphi_{2(\alpha)}=\left(\sigma_{\alpha}^{r} \widetilde{\sigma}_{\alpha}\right) \varphi_{1(\alpha)}, \hat{\sigma}_{\alpha}^{r} \varphi_{2(\alpha)}=\sigma_{\alpha}^{r} \varphi_{1(\alpha)}=\left(\sigma_{\alpha}^{r} \widetilde{\sigma}_{\alpha}\right) \varphi_{2(\alpha)}$. Whence, the single eigenvalue $\left(\sigma_{\alpha}^{r} \widetilde{\sigma}_{\alpha}\right)$ associates with different $\varphi_{\lambda(\alpha)}$, namely it is degenerate with degeneracy degree equal 2. Thus, among quantum numbers $r$ there is also the quantum number of the half integer spin $\vec{\sigma}\left(\sigma_{3}=\frac{1}{2} s, \quad s= \pm 1\right)$. This consequently gives rise to the spins of particles. The one occupied state reads $\varphi_{r^{\prime}(\alpha)}=\left(\varphi_{2(\alpha)}\right)_{r^{\prime}} \prod_{r \neq r^{\prime}}\left(\chi_{1}\right)_{r}$. Next, we introduce the operators $\hat{\gamma}_{(\lambda, \mu, \alpha)}^{r} \equiv \hat{O}_{\lambda}^{r_{1}} \otimes \hat{O}_{\mu}^{r_{2}} \otimes \hat{\sigma}_{\alpha}^{r_{3}}$ 
and the state vectors $\chi_{\lambda, \mu, \tau(\alpha)} \equiv \mid \lambda, \mu, \tau(\alpha)>=\chi_{\lambda} \otimes \chi_{\mu} \otimes \varphi_{\tau(\alpha)}$, where $\lambda, \mu, \tau, \nu=1,2 ; \quad \alpha, \beta=1,2,3$ and $r \equiv\left(r_{1}, r_{2}, r_{3}\right)$. Omitting two valuedness of state vectors we apply $|\lambda, \tau, \delta(\beta)>\equiv| \lambda, \tau>$, and remember that always the summation must be extended over the double degeneracy of the spin states $(s= \pm 1)$. The explicit matrix elements of basis vectors read $<\lambda, \mu\left|\hat{\gamma}_{(\tau, \nu, \alpha)}^{r}\right| \tau, \nu>={ }^{*} \delta_{\lambda \tau}{ }^{*} \delta_{\mu \nu} e_{(\tau, \nu, \alpha)}^{r}$, $<\tau, \nu\left|\hat{\gamma}_{r}^{(\tau, \nu, \alpha)}\right| \lambda, \mu>={ }^{*} \delta_{\lambda \tau}{ }^{*} \delta_{\mu \nu} e_{r}^{(\tau, \nu, \alpha)}$, for given $\lambda, \mu$. The operators of occupation numbers $\hat{N}_{1}^{r r^{\prime}}=$ $\hat{\gamma}_{(1,1, \alpha)}^{r} \hat{\gamma}_{(2,2, \beta)}^{r^{\prime}}, \hat{N}_{2}^{r r^{\prime}}=\hat{\gamma}_{(2,1, \alpha)}^{r} \hat{\gamma}_{(1,2, \beta)}^{r^{\prime}}$ have the expectation values implying Pauli's exclusion principle $<2,2\left|\hat{N}_{1}^{r r^{\prime}}\right| 2,2>=<1,2\left|\hat{N}_{2 \alpha \beta}^{r r^{\prime}}\right| 1,2>=\delta_{r r^{\prime}} \delta_{\alpha \beta},<1,1\left|\hat{N}_{1 \alpha \beta}^{r r^{\prime}}\right| 1,1>=<2,1\left|\hat{N}_{2} r r_{\alpha \beta}^{\prime}\right| 2,1>=0$. The operators $\left\{\hat{\gamma}^{r}\right\}$ are the basis for tangent operator vectors $\hat{\Phi}(\zeta)=\hat{\gamma}^{r} \Phi_{r}(\zeta)$ of the 12 dimensional flat OM: $\hat{G}$, where we introduce the vector function belonging to the ordinary class of functions of $C^{\infty}$ smoothness defined on the manifold $G: \quad \Phi_{r}^{(\lambda, \mu, \alpha)}(\zeta)=\zeta^{(\lambda, \mu, \alpha)} \Phi_{r}^{\lambda, \mu}(\zeta), \quad \zeta \in G$. But, the operators $\left\{\hat{\gamma}_{r}\right\}$ is a dual basis for operator covectors $\overline{\hat{\Phi}}(\zeta)=\hat{\gamma}_{r} \Phi^{r}(\zeta)$, where $\Phi^{r}=\bar{\Phi}_{r}$ (charge conjugated). Hence $<\lambda, \mu|\hat{\Phi}(\zeta) \overline{\hat{\Phi}}(\zeta)| \lambda, \mu>={ }^{*} \delta_{\lambda \tau}{ }^{*} \delta_{\mu \nu} \Phi_{r}^{(\tau, \nu, \alpha)}(\zeta) \Phi_{(\tau, \nu, \alpha)}^{r}(\zeta)$, for given $\lambda, \mu$. Considering the state vectors $\mid \chi_{ \pm}>$, we get the matrix elements $<\chi_{+}|\hat{\Phi}(\zeta) \hat{\hat{\Phi}}(\zeta)| \chi_{+}>\equiv \Phi_{+}^{2}(\zeta)=\Phi_{r}^{(\lambda, 1, \alpha)}(\zeta) \Phi_{(\lambda, 1, \alpha)}^{r}(\zeta)$, $<\chi_{-}|\hat{\Phi}(\zeta) \overline{\hat{\Phi}}(\zeta)| \chi_{-}>\equiv \Phi_{-}^{2}(\zeta)=\Phi_{r}^{(\lambda, 2, \alpha)}(\zeta) \Phi_{(\lambda, 2, \alpha)}^{r}(\zeta)$. The basis $\left\{\hat{\gamma}^{r}\right\}$ decomposes into $\left\{\hat{\gamma}_{i}^{r}\right\}(i=$ $\eta, u)$, where $\hat{\gamma}_{i(+\alpha)}^{r}=\frac{1}{\sqrt{2}}\left(\gamma_{(1,1 \alpha)}^{r}+\varepsilon_{i} \gamma_{(2,1 \alpha)}^{r}\right), \hat{\gamma}_{i(-\alpha)}^{r}=\frac{1}{\sqrt{2}}\left(\gamma_{(1,2 \alpha)}^{r}+\varepsilon_{i} \gamma_{(2,2 \alpha)}^{r}\right)$. The expansion of operator vectors $\hat{\Psi}_{i} \in \hat{G}_{i}$ and operator covectors $\hat{\Psi}_{i}$ are written $\hat{\Psi}_{i}=\hat{\gamma}_{i}{ }^{r} \Psi_{i r}, \hat{\hat{\Psi}}_{i}=\hat{\gamma}_{i r} \Psi_{i}{ }^{r}$, where the following vector functions of $C^{\infty}$ smoothness are defined on the manifolds $G_{i}: \Psi_{\eta r}{ }^{( \pm \alpha)}\left(\eta, p_{\eta}\right)=\eta^{( \pm \alpha)} \Psi_{\eta r}{ }^{ \pm}\left(\eta, p_{\eta}\right)$, $\Psi_{u r}{ }^{( \pm \alpha)}\left(u, p_{u}\right)=u^{( \pm \alpha)} \Psi_{u r}^{ \pm}\left(u, p_{u}\right)$. Namely, the probability of finding the vector function in the state $r$ with given sixvector of coordinate $(\eta$ or $u)$ and momentum $\left(p_{\eta}\right.$ or $\left.p_{u}\right)$ is determined by the square of its state wave function $\Psi_{\eta}^{ \pm}\left(\eta, p_{\eta}\right)$, or $P s i_{u}^{ \pm}\left(u, p_{u}\right)$. Due to the spin states, the $\Psi_{i r}^{ \pm}$can be regarded as the Fermi field of the positive and negative frequencies $\Psi_{i r}^{ \pm} \equiv \Psi_{i \pm p}^{r}$.

\subsection{Realization of the Flat Manifold $G$}

The bispinor $\Psi(\zeta)$ defined on the manifold $G=G_{\eta} \oplus G_{u}$ can be written $\Psi(\zeta)=\Psi_{\eta}(\eta) \Psi_{u}(u)$, where $\Psi_{i}$ is the bispinor defined on the manifold $G_{i}$. The free state of $i$-type fermion with definite values of momentum $p_{i}$ and spin projection $s$ is described by plane waves. The relations of orthogonality and completeness hold for the spinors. Considering also the solutions of negative frequencies, we make use of localized wave packets constructed by means of superposition of plane wave solutions furnished by creation and annihilation operators in agreement with Pauli's principle $\hat{\Psi}_{i}=\sum_{ \pm s} \int \frac{d^{3} p_{i}}{(2 \pi)^{3 / 2}}\left(\hat{\gamma}_{i(+\alpha)} \Psi_{i}^{(+\alpha)}+\hat{\gamma}_{i(-\alpha)} \Psi_{i}^{(-\alpha)}\right)$, etc, where the summation is extended over all dummy indices. In such a manner we can treat as well the wave packets of operator vector fields $\hat{\Phi}(\zeta)$. While the matrix element of the anticommutator of expansion coefficients reads $<\chi_{-}\left|\left\{\hat{\gamma}_{i}^{(+\alpha)}\left(p_{i}, s\right), \hat{\gamma}_{j(+\beta)}\left(p_{j}^{\prime}, s^{\prime}\right)\right\}\right| \chi_{-}>=\varepsilon_{i} \delta_{i j} \delta_{s s^{\prime}} \delta_{\alpha \beta} \delta^{(3)}\left(\vec{p}_{i}-\vec{p}_{i}^{\prime}\right)$. In the aftermath, we get the most important relation $\sum_{\lambda= \pm}<\chi_{\lambda}|\hat{\Phi}(\zeta) \overline{\hat{\Phi}}(\zeta)| \chi_{\lambda}>=\sum_{\lambda= \pm}<\chi_{\lambda}|\overline{\hat{\Phi}}(\zeta) \hat{\Phi}(\zeta)| \chi_{\lambda}>=-i \zeta^{2} G_{\zeta}(0)=$ $-i\left(\eta^{2} G_{\eta}(0)-u^{2} G_{u}(0)\right)$, where $G_{i}(0) \equiv \lim _{i \rightarrow i^{\prime}} G_{i}\left(i-i^{\prime}\right), \quad(i=\zeta, \eta, u$,$) , etc., the Green's function$ $G_{i}\left(i-i^{\prime}\right)=-\left(i \hat{\partial}_{i}+m\right) \Delta_{i}\left(i-i^{\prime}\right)$ is provided by the usual invariant singular functions $\Delta_{i}\left(i-i^{\prime}\right)$. Realization of the flat manifold $G$ ensued from the constraint imposed upon the matrix element that, as the geometric object, it is required to be finite $\sum_{\lambda= \pm}<\chi_{\lambda}|\hat{\Phi}(\zeta) \overline{\hat{\Phi}}(\zeta)| \chi_{\lambda}><\infty$, which gives rise to $\zeta^{2} G_{\zeta F}(0)<\infty$, and $G_{\zeta F}(0)=G_{\eta F}(0)=G_{u F}(0)=\lim _{u \rightarrow u^{\prime}}\left[-i \sum_{\vec{p}_{u}} \Psi_{u p_{u}}(u) \bar{\Psi}_{u p_{u}}\left(u^{\prime}\right) \theta\left(u_{0}-\right.\right.$ $\left.\left.u_{0}^{\prime}\right)+i \sum_{\vec{p}_{u}} \bar{\Psi}_{u-p_{u}}\left(u^{\prime}\right) \Psi_{u-p_{u}}(u) \theta\left(u_{0}^{\prime}-u_{0}\right)\right)$, where the $G_{\zeta F}, G_{\eta F}$ and $G_{u F}$ are causal Green's functions characterized by the boundary condition that only positive frequency occur for $\eta_{0}>0 \quad\left(u_{0}>0\right)$, only negative for $\eta_{0}<0 \quad\left(u_{0}<0\right)$. Here $\eta_{0}=\left|\vec{\eta}_{0}\right|, \eta_{0 \alpha}=\frac{1}{\sqrt{2}}\left(\eta_{(+\alpha)}+\eta_{(-\alpha)}\right)$ and the same holds for $u_{0}$. Satisfying the condition above, a length of each vector $\zeta=e \zeta \in G$ compulsory must be equaled zero $\zeta^{2}=\eta^{2}-u^{2}=0$, which is the condition of realization of the flat manifold $G$. The latter subsequently leads to Minkowski flat space $M_{4}$, the relativity principle holds $\left.d \eta^{2}\right|_{6 \rightarrow 4} \equiv d s^{2}=d u^{2}=i n v$. 


\section{OM: Field aspect}

The quantum field theory of the OM is equivalent to configuration space wave mechanics employing the antisymmetric state functions incorporated with geometric properties of corresponding objects. Therein, by applying the algebraic approach we reach to rigorous definition of the OM: $\hat{G}$, construct the explicit forms of wave state functions and calculate the matrix elements of field operators. While, the $\hat{G}$ reads $\hat{G}=\sum_{n=0}^{\infty} \hat{G}^{(n)}=\sum_{n=0}^{\infty}\left(\hat{\mathcal{U}}^{(n)} \otimes \overline{\mathcal{H}}^{(n)}\right)$, where $\hat{\mathcal{U}}_{\left(r_{1}, \ldots, r_{n}\right)}^{(n)}=\hat{\mathcal{U}}_{r_{1}}^{(1)}, \otimes \cdots \otimes \hat{\mathcal{U}}_{r_{n}}^{(1)}$ is the open neighbourhood of the n-points $\hat{\zeta}_{r_{i}}$ of the OM, $\overline{\mathcal{H}}_{\left(r_{1}, \ldots, r_{n}\right)}^{(n)}=\mathcal{H}_{r_{1}}^{(1)} \otimes \cdots \otimes \mathcal{H}_{r_{n}}^{(1)}$ is the Hilbert space for description of $\mathrm{n}$ particle system. To illustrate the point at issue, the operators $\left\{\hat{\gamma}^{r}\right\}$ are the basis for tangent operator vectors $\hat{\Phi}(\zeta)=\hat{\gamma}^{r} \Phi_{r}(\zeta)$ of the 12D flat OM: $\hat{G}$, where we introduce the vector function belonging to the ordinary class of functions of $C^{\infty}$ smoothness defined on the 12D manifold $G: \quad \Phi_{r}^{(\lambda, \mu, \alpha)}(\zeta)=\zeta^{(\lambda, \mu, \alpha)} \Phi_{r}^{\lambda, \mu}(\zeta), \quad \zeta \in G(\lambda, \mu=1,2 ; \alpha=1,2,3)$. But, the operators $\left\{\hat{\gamma}_{r}\right\}$ is a dual basis for operator covectors $\hat{\Phi}(\zeta)=\hat{\gamma}_{r} \Phi^{r}(\zeta)$, where $\Phi^{r}=\bar{\Phi}_{r}$ (charge conjugated). The explicit matrix elements of basis vectors read $<\lambda, \mu\left|\hat{\gamma}_{(\tau, \nu, \alpha)}^{r}\right| \tau, \nu>={ }^{*} \delta_{\lambda \tau}{ }^{*} \delta_{\mu \nu} e_{(\tau, \nu, \alpha)}^{r}, \quad<\tau, \nu\left|\hat{\gamma}_{r}^{(\tau, \nu, \alpha)}\right|$ $\lambda, \mu>={ }^{*} \delta_{\lambda \tau}{ }^{*} \delta_{\mu \nu} e_{r}^{(\tau, \nu, \alpha)}$, for given $\lambda, \mu$, and ${ }^{*} \delta_{\lambda \tau}=1-\delta_{\lambda \tau}$. The operators of occupation numbers $\hat{N}_{1 \alpha \beta}^{r r^{\prime}}=\hat{\gamma}_{(1,1, \alpha)}^{r} \hat{\gamma}_{(2,2, \beta)}^{r^{\prime}}, \quad \hat{N}_{2 \alpha \beta}^{r r^{\prime}}=\hat{\gamma}_{(2,1, \alpha)}^{r} \hat{\gamma}_{(1,2, \beta)}^{r^{\prime}}$, have the expectation values implying Pauli's exclusion principle $<2,2\left|\hat{N}_{1}^{r r^{\prime}}\right| 2,2>=<1,2\left|\hat{N}_{2}^{r r^{\prime}}\right| 1,2>=\delta_{r r^{\prime}} \delta_{\alpha \beta}, \quad<1,1\left|\hat{N}_{1}^{r r^{\prime}}\right| 1,1>=<2,1\left|\hat{N}_{2}^{r r^{\prime}} r\right|$ $2,1>=0$. And $<\lambda, \mu|\hat{\Phi}(\zeta) \overline{\hat{\Phi}}(\zeta)| \lambda, \mu>={ }^{*} \delta_{\lambda \tau}{ }^{*} \delta_{\mu \nu} \Phi_{r}^{(\tau, \nu, \alpha)}(\zeta) \Phi_{(\tau, \nu, \alpha)}^{r}(\zeta)$, for given $\lambda, \mu$. Meanwhile, one has to modify the basis operators (the creation $\hat{\gamma}_{r} \rightarrow \hat{\gamma}_{r_{i}}$ and annihilation $\hat{\gamma}^{r} \rightarrow \hat{\gamma}^{r_{i}}$ operators) in order to provide an anticommutation in arbitrary $\left(\mid>_{r_{i}}\right)$ states. For example, acting on free state $\mid 0>_{r_{i}}$ the creation operator $\hat{\gamma}_{r_{i}}$ now yields the one occupied state $\mid 1>_{r_{i}}$ with the phase ${ }^{\prime}{ }^{\prime}$ or $^{\prime}-^{\prime}$ depending of parity of the number of quanta in the states $r<r_{i}$. Modified operators satisfy the same anticommutation relations of the basis operators. It is convenient to make use of notation $\hat{\gamma}_{r}^{(\lambda, \mu, \alpha)} \equiv e_{r}^{(\lambda, \mu, \alpha)} \hat{b}_{(r \alpha)}^{\lambda \mu}$, and abbreviate the pair of indices $(r \alpha)$ by the single symbol $r$. Then for each $\Phi \in{ }^{A} \mathcal{H}^{(n)}$ and any vector $f \in$ $\mathcal{H}^{(1)}$ the operators $\hat{b}(f)$ and $\hat{b}^{*}(f)$ imply $\hat{b}(f) \Phi=\frac{1}{\sqrt{(n-1) !}} \sum_{\sigma \in S(n)} \operatorname{sgn}(\sigma)\left(f \Phi_{\sigma(1)}^{(1)}\right) \Phi_{\sigma(2)}^{(1)} \otimes \cdots \otimes \Phi_{\sigma(n)}^{(1)}$, $\hat{b}^{*}(f) \Phi=\frac{1}{\sqrt{(n+1) !}} \sum_{\sigma \in S(n+1)} \operatorname{sgn}(\sigma) \Phi_{\sigma(0)}^{(1)} \otimes \Phi_{\sigma(1)}^{(1)} \otimes \cdots \otimes \Phi_{\sigma(n)}^{(1)}$, where $\Phi_{(0)}^{(1)} \equiv f$. One continues the $\hat{b}(f)$ and $\hat{b}^{*}(f)$ by linearity to linear reflections, which are denoted by the same symbols acting respectively from ${ }^{A} \mathcal{H}^{(n)}$ onto ${ }^{A} \mathcal{H}^{(n-1)}$ or ${ }^{A} \mathcal{H}^{(n+1)}$. They are limited over the values $\sqrt{n}|f|$ and $\sqrt{(n+1)}|f|$ and can be expanded by continuation up to the reflections acting from ${ }^{A} \overline{\mathcal{H}}^{(n)}$ onto ${ }^{A} \overline{\mathcal{H}}^{(n-1)}$ or ${ }^{A} \overline{\mathcal{H}}^{(n+1)}$. Finally, they must be continued by linearity up to the linear operators acting from ${ }^{A} \mathcal{F}$ onto ${ }^{A} \mathcal{F}$ defined on the same closed region in ${ }^{A} \overline{\mathcal{H}}^{(n)}$, namely in ${ }^{A} \mathcal{F}$, which is invariant with respect to reflections $\hat{b}(f)$ and $\hat{b}^{*}(f)$. Hence, at $f_{i}, g_{i} \in \mathcal{H}^{(1)}(i=1, \ldots, n ; j=1, \ldots, m)$ all polynomials over $\left\{\hat{b}^{*}\left(f_{i}\right)\right\}$ and $\left\{\hat{b}\left(g_{j}\right)\right\}$ are completely defined on ${ }^{A} \mathcal{F}$. While, for given $\lambda, \mu$, one has $\left\langle\lambda, \mu\left|\left\{\hat{b}_{r}^{\lambda \mu}(f), \hat{b}_{\lambda \mu}^{r^{\prime}}(g)\right\}\right| \lambda, \mu>=\delta_{r}^{r^{\prime}}\right.$. The mean values $<\varphi ; \hat{b}_{r}^{\lambda \mu}(f) \hat{b}_{\lambda \mu}^{r}(f)>$ calculated at fixed $\lambda, \mu$ for any element $\Phi \in{ }^{A} \mathcal{F}$ equal to mean values of the symmetric operator of occupation number in terms of $\hat{N}^{r}=\hat{b}_{r}(f) \hat{b}^{r}(f)$, with a wave function $f$ in the state described by $\Phi$. Here, as usual, it is denoted $\langle\varphi ; A \Phi\rangle=\operatorname{Tr} \operatorname{P}_{\varphi} A=(\Phi, A \Phi)$ for each vector $\Phi \in \mathcal{H}$ with $|\Phi|=1$, while the $P_{\varphi}$ is projecting operator onto one dimensional space $\{\lambda \Phi \mid \lambda \in C\}$ generated by $\Phi$. Therewith, the probability of transition $\varphi \rightarrow \psi$ is given $\operatorname{Pr}\{\varphi \mid \psi\}=|(\psi, \varphi)|^{2}$. The linear operator $A$ defined on the elements of linear manifold $\mathcal{D}(A)$ of $\mathcal{H}$ takes the values in $\mathcal{H}$. The $\mathcal{D}(A)$ is an overall closed region of definition of $A$, namely the closure of $\mathcal{D}(A)$ by the norm given in $\mathcal{H}$ coincides with $\mathcal{H}$. Meanwhile, the $\mathcal{D}(A)$ included in $\mathcal{D}\left(A^{*}\right)$ and $A$ coincides with the reduction of $A^{*}$ on $\mathcal{D}(A)$, because $\mathcal{D}(A)$ is the symmetric operator such that the linear operator $A^{*}$ is the maximal conjugated to $A$. That is, any operator $A^{\prime}$ conjugated to $A-\left(\Psi, A^{\prime} \Phi\right)=\left(A^{\prime} \Psi, \Phi\right)$ for all $\Phi \in \mathcal{D}(A)$ and $\Psi \in \mathcal{D}\left(A^{\prime}\right)$ coincides with the reduction of $A^{*}$ on some linear manifold $\mathcal{D}\left(A^{\prime}\right)$ included in $\mathcal{D}\left(A^{*}\right)$. Thus, the operator $A^{* *}$ is closed symmetric expansion of operator $A$, namely it is a closure of $A$. Self conjugated operator $A$, the closure of which is self conjugated as well, allows only the one self conjugated expansion $A^{* *}$. Hence, self conjugated closure $\hat{N}$ of operator $\sum_{i=1}^{\infty} \hat{b}^{*}\left(f_{i}\right) \hat{b}\left(f_{i}\right)$, where $\left\{f_{i} \mid i=1, \ldots, n\right\}$ is an arbitrary orthogonal basis on $\mathcal{H}^{(1)}$, can be regarded as the operator of occupation number. For the vector $\chi^{0} \in{ }^{A} \mathcal{F}$ and $\chi^{0(n)}=\delta_{0 n}$ one gets $<\chi^{0(n)}, \hat{N}(f)>=0$ for all $f \in \mathcal{H}^{(1)}$. Thus, $\chi^{0}$ is the 
vector of vacuum state: $\hat{b}(f) \chi^{0}=0$ for all $f \in \mathcal{H}^{(1)}$. If $f=\left\{f_{i} \mid i=1,2, \ldots\right\}$ is an arbitrary orthogonal basis on $\mathcal{H}^{(1)}$, then due to irreducibility of operators $\hat{b}^{*}\left(f_{i}\right) \mid f_{i} \in f$, the ${ }^{A} \mathcal{H}$ includes the 0 and whole space ${ }^{A} \mathcal{H}$ as invariant subspaces with respect to all $\hat{b}^{*}(f)$. To define the 12 dimensional operator manifold $\hat{G}$ we consider a set $\hat{\mathcal{F}}$ of all the sequences $\hat{\Phi}=\left\{\hat{\Phi}^{(0)}, \hat{\Phi}^{(1)}, \ldots, \hat{\Phi}^{(n)}, \ldots\right\}$ with a finite number of nonzero elements provided by $\hat{\Phi}_{\left(r_{1}, \ldots, r_{n}\right)}^{(n)}=\hat{\Phi}_{r_{1}}^{(1)} \otimes \cdots \otimes \hat{\Phi}_{r_{n}}^{(1)} \in \hat{G}^{(n)}, \hat{\Phi}_{r_{i}}^{(1)}=\hat{\zeta}_{r_{i}} \Phi_{r_{i}}^{(1)} \in \hat{G}_{i}^{(1)}=\hat{\mathcal{U}}_{i}^{(1)} \otimes \mathcal{H}_{i}^{(1)}$, $\hat{\zeta}_{r_{i}} \equiv \sum_{\alpha_{i}=1}^{3} \hat{\gamma}_{\left(\lambda_{i}, \mu_{i}, \alpha_{i}\right)}^{r_{i}} \zeta_{r_{i}}^{\left(\lambda_{i}, \mu_{i}, \alpha_{i}\right)} \in \hat{\mathcal{U}}_{r_{i}}^{(1)}, \hat{G}^{(n)}=\hat{\mathcal{U}}^{(n)} \otimes \overline{\mathcal{H}}^{(n)}, \hat{\mathcal{U}}_{\left(r_{1}, \ldots, r_{n}\right)}^{(n)}=\hat{\mathcal{U}}_{r_{1}}^{(1)}, \otimes \cdots \otimes \hat{\mathcal{U}}_{r_{n}}^{(1)}$. Then, the operator manifold $\hat{G}$ ensues $\hat{G}=\sum_{n=0}^{\infty} \hat{G}^{(n)}=\sum_{n=0}^{\infty}\left(\hat{\mathcal{U}}^{(n)} \otimes \overline{\mathcal{H}}^{(n)}\right)$. To define the secondary quantized form of one particle observable $A$ on $\mathcal{H}$, let consider a set of identical samples $\hat{\mathcal{H}}_{i}$ of one particle space $\mathcal{H}^{(1)}$ and operators $A_{i}$ acting on them. To each closed linear operator $A^{(1)}$ in $\mathcal{H}^{(1)}$ with overall closed region of definition $\mathcal{D}\left(A^{(1)}\right)$ following operators are corresponded: $A_{1}^{(n)}=A^{(1)} \otimes I \otimes \cdots \otimes I, \ldots$, $A_{n}^{(n)}=I \otimes I \otimes \cdots \otimes A^{(1)}$. The $\operatorname{sum} \sum_{j=1}^{n} A_{j}^{(n)}$ is given on the intersection of regions of definition of operator terms including a linear manifold $\mathcal{D}\left(A^{(1)}\right) \otimes \cdots \otimes \mathcal{D}\left(A^{(n)}\right)$ closed in $\hat{\mathcal{H}}^{(n)}$. While, the $A^{(n)}$ is a minimal closed expansion of this sum with $\mathcal{D}\left(A^{(n)}\right)$. One considers a linear manifold $\mathcal{D}(\Omega(A))$ in $\mathcal{H}=\sum_{n=0}^{\infty} \hat{\mathcal{H}}^{(n)}$ defined as a set of all the vectors $\Psi \in \mathcal{H}$ such as $\Psi^{(n)} \in \mathcal{D}\left(A^{(n)}\right)$ and $\sum_{n=0}^{\infty}\left|A^{(n)} \Psi^{(n)}\right|^{2}<\infty$. The manifold $\mathcal{D}(\Omega(A))$ is closed in $\mathcal{H}$. On this manifold one defines a closed linear operator $\Omega(A)$ acting as $\Omega(A)^{(n)}=A^{(n)} \Psi^{(n)}$, namely $\Omega(A) \Phi=\sum_{n=0}^{\infty} A^{(n)} \Psi^{(n)}$, while the $\Omega(A)$ is self conjugated operator with overall closed region of definition, where $\Phi_{i} \in \mathcal{D}(A)$. Then, $\left\langle\varphi^{(n)} ; A^{(n)}\right\rangle=\sum_{i=1}^{n}\left\langle\varphi_{i} ; A>\right.$, which enables the expansion by continuing onto $\mathcal{D}(A)$. Thus, $A^{(n)}$ is the $n$ particle observable corresponding to one particle observable $A$. So $<\varphi ; \Omega(A)>=\sum_{n=0}^{\infty}<\varphi^{(n)} ; A^{(n)}>$ for any $\Phi_{i} \in \mathcal{D}(\Omega(A))$. While, the $\Omega(A)$ reflects ${ }^{A} \mathcal{D}=\mathcal{D}(\Omega(A)) \frown{ }^{A} \mathcal{H}$ into ${ }^{A} \mathcal{H}$. The reduction of $\Omega(A)$ on ${ }^{A} \mathcal{H}$ is the self conjugated in the region ${ }^{A} \mathcal{D}$, because ${ }^{A} \mathcal{H}$ is the closed subspace of the $\mathcal{H}$. Hence, the $\Omega(A)$ is the secondary quantized form of one particle observable $A$ on the $\mathcal{H}$. The vacuum state reads $\chi^{0}\left(\nu_{1}, \nu_{2}, \nu_{3}, \nu_{4}\right)=\mid 1,1>^{\nu_{1}}$

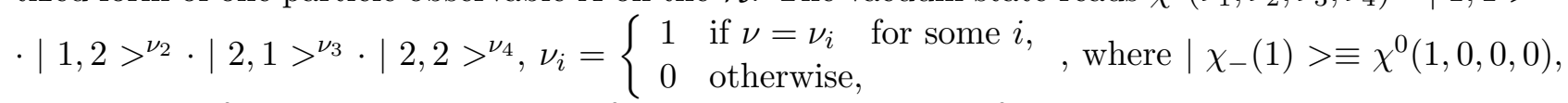
$\left|\chi_{+}(1)>\equiv \chi^{0}(0,0,0,1),\right| \chi_{-}(2)>\equiv \chi^{0}(0,0,1,0),\left|\chi_{+}(2)>\equiv \chi^{0}(0,1,0,0),<\chi_{ \pm}(\lambda)\right| \chi_{ \pm}(\mu)>=\delta_{\lambda \mu}$, and $<\chi_{ \pm}(\lambda) \mid \chi_{\mp}(\mu)>=0$, provided by $<\chi_{ \pm}|A| \chi_{ \pm}>\equiv \sum_{\lambda}<\chi_{ \pm}(\lambda)|A| \chi_{ \pm}(\lambda)>$ and the normalization condition $<\chi^{0}\left(\nu_{1}^{\prime}, \nu_{2}^{\prime}, \nu_{3}^{\prime}, \nu_{4}^{\prime}\right) \mid \chi^{0}\left(\nu_{1}, \nu_{2}, \nu_{3}, \nu_{4}\right)>=\prod_{i=1}^{4} \delta_{\nu_{i} \nu_{i}^{\prime}}$. The state vectors are introduced $\chi\left(\left\{n_{r}\right\}_{1}^{N} ;\left\{m_{r}\right\}_{1}^{M} ;\left\{q_{r}\right\}_{1}^{Q} ;\left\{t_{r}\right\}_{1}^{T} ;\left\{\nu_{r}\right\}_{1}^{4}\right)=\left(\hat{b}_{N}^{11}\right)^{n_{N}} \cdots\left(\hat{b}_{1}^{11}\right)^{n_{1}} \cdot\left(\hat{b}_{M}^{12}\right)^{m_{M}} \cdots\left(\hat{b}_{1}^{12}\right)^{m_{1}}$. $\left(\hat{b}_{Q}^{21}\right)^{q_{Q}} \cdots\left(\hat{b}_{1}^{21}\right)^{q_{1}} \cdots\left(\hat{b}_{T}^{22}\right)^{t_{T}} \cdots\left(\hat{b}_{1}^{22}\right)^{t_{1}} \chi^{0}\left(\nu_{1}, \nu_{2}, \nu_{3}, \nu_{4}\right)$, where $\left\{n_{r}\right\}_{1}^{N}=n_{1}, \ldots, n_{N}$, etc., which are the eigenfunctions of modified operators. They form a whole set of orthogonal vectors $\left\langle\chi, \chi^{\prime}\right\rangle=$ $\prod_{r=1}^{N} \delta_{n_{r} n_{r}^{\prime}} \cdot \prod_{r=1}^{M} \delta_{m_{r} m_{r}^{\prime}} \cdot \prod_{r=1}^{Q} \delta_{q_{r} q_{r}^{\prime}} \cdot \prod_{r=1}^{T} \delta_{t_{r} t_{r}^{\prime}} \cdot \prod_{r=1}^{4} \delta_{\nu_{r} \nu_{r}^{\prime}}$. Considering an arbitrary superposition $\chi=$ $\sum_{a=\left\{n_{r}\right\}_{1}^{N},\left\{m_{r}\right\}_{1}^{M},\left\{q_{r}\right\}_{1}^{Q},\left\{t_{r}\right\}_{1}^{T}=0}^{1} c^{\prime}(a) \chi(a)$, the coefficients $c^{\prime}$ of expansion are the corresponding amplitudes of probabilities. The nonvanishing matrix elements of operators $\hat{b}_{r_{k}}^{11}$ and $\hat{b}_{11}^{r_{k}}$ read $<\chi\left(\left\{n_{r}^{\prime}\right\}_{1}^{N} ; 0 ; 0 ; 0 ; 1,0,0,0\right)\left|\hat{b}_{r_{k}}^{11} \chi\left(\left\{n_{r}\right\}_{1}^{N} ; 0 ; 0 ; 0 ; 1,0,0,0\right)>=<1,1\right| \hat{b}_{11}^{r_{1}^{\prime}} \cdots \hat{b}_{11}^{r_{n}^{\prime}} \cdot \hat{b}_{r_{k}}^{11} \cdot \hat{b}_{r_{n}}^{11} \cdots \hat{b}_{r_{1}}^{11} \mid 1,1>=$ $\begin{cases}(-1)^{n^{\prime}-k^{\prime}} & \text { if } n_{r}=n_{r}^{\prime} \text { for } r \neq r_{k} \text { and } n_{r_{k}}=0 ; n_{r_{k}}^{\prime}=1, \\ 0 & \text { otherwise, }\end{cases}$ $\sum_{r=1}^{N} n_{r}^{\prime}$, the $r_{k}$ and $r_{k}^{\prime}$ are $k$-th and $k^{\prime}$-th terms of regulated sets of $\left\{r_{1}, \ldots, r_{n}\right\} \quad\left(r_{1}<r_{2}<\cdots<r_{n}\right)$ and $\left\{r_{1}^{\prime}, \ldots, r_{n}^{\prime}\right\} \quad\left(r_{1}^{\prime}<r_{2}^{\prime}<\cdots<r_{n}^{\prime}\right)$, respectively. Continuing along this line we get a whole set of explicit forms of matrix elements of the rest of operators $\hat{b}_{r_{k}}$ and $\hat{b}^{r_{k}}$. Hence $\sum_{\left\{\nu_{r}\right\}=0}^{1}<\chi^{0} \mid$ $\hat{\Phi}(\zeta) \mid \chi>=\sum_{r=1}^{N} c_{n_{r}}^{\prime} e_{(1,1, \alpha)}^{n_{r}} \Phi_{n_{r}}^{(1,1, \alpha)}+\cdots$, provided by $c_{n_{r}}^{\prime} \equiv \delta_{1 n_{r}} c^{\prime}\left(0, \ldots, n_{r}, \ldots, 0 ; 0 ; 0 ; 0\right), \cdots$ Hereinafter we change a notation of the coefficients $\bar{c}\left(r^{11}\right)=c_{n_{r}}^{\prime}, \bar{c}\left(r^{21}\right)=c_{q_{r}}^{\prime}, \quad N_{11}=N, N_{21}=Q$, $\bar{c}\left(r^{12}\right)=c_{m_{r}}^{\prime}, \bar{c}\left(r^{22}\right)=c_{t_{r}}^{\prime}, N_{12}=M, N_{22}=T$, and make use of convention $F_{r^{\lambda \mu}}=\sum_{\alpha} e_{(\lambda, \mu, \alpha)}^{r^{\lambda \mu}} \Phi_{r^{\lambda \mu}}^{(\lambda, \mu, \alpha)}$, $\sum_{\left\{\nu_{r}\right\}=0}^{1}<\chi^{0}|\hat{A}| \chi>\equiv<\chi^{0}\|\hat{A}\| \chi>$, The matrix elements of operator vector and covector fields take the final forms $<\chi^{0}\|\hat{\Phi}(\zeta)\| \chi>=\sum_{\lambda \mu=1}^{2} \sum_{r^{\lambda \mu}=1}^{N_{\lambda \mu}} \bar{c}\left(r^{\lambda \mu}\right) F_{r^{\lambda \mu}}(\zeta),<\chi\|\bar{\Phi}(\zeta)\| \chi^{0}>=$ $\sum_{\lambda \mu=1}^{2} \sum_{r^{\lambda \mu}=1}^{N_{\lambda \mu}} \bar{c}^{*}\left(r^{\lambda \mu}\right) F^{r^{\lambda \mu}}(\zeta)$. In the following we shall use a convention: $\left\{\sum_{\lambda \mu}^{2}\right\}_{1}^{n} \equiv \sum_{\lambda_{1} \mu_{1}}^{2} \ldots \sum_{\lambda_{n} \mu_{n}}^{2}$, $r_{i}^{\lambda \mu} \equiv r^{\lambda_{i} \mu_{i}}$ and $\bar{c}\left(r_{1}^{11}, \ldots, r_{n}^{11}\right)=c^{\prime}\left(n_{1}, \ldots, n_{N} ; 0 ; 0 ; 0\right)$, etc. The anticommutation relations ensue $<$ $\chi_{-}\left|\left\{\hat{b}_{i r}^{+}, \hat{b}_{i+}^{r^{\prime}}\right\}\right| \chi_{-}>=<\chi_{+}\left|\left\{\hat{b}_{i r}^{-}, \hat{b}_{i}^{r_{-}^{\prime}}\right\}\right| \chi_{+}>=\delta_{r}^{r^{\prime}}$, provided by $\hat{\gamma}_{i r}^{(\lambda \alpha)}=\hat{e}_{i r}{ }^{(\lambda \alpha)} \hat{b}_{i}^{\lambda}{ }_{(r \alpha)}, \quad(r \alpha) \rightarrow r$. 
The state functions $\chi=\left(\hat{b}_{\eta N}^{+}\right)^{n_{N}} \cdots\left(\hat{b}_{\eta 1}^{+}\right)^{n_{1}} \cdot\left(\hat{b}_{\eta M}^{-}\right)^{m_{M}} \cdots\left(\hat{b}_{\eta 1}^{-}\right)^{m_{1}} \cdot\left(\hat{b}_{u Q}^{+}\right)^{q_{Q}} \cdots\left(\hat{b}_{u 1}^{+}\right)^{q_{1}} \cdot\left(\hat{b}_{u T}^{-}\right)^{t_{T}} \cdots\left(\hat{b}_{u 1}^{-}\right)^{t_{1}}$. $\chi_{-}(\lambda) \chi_{+}(\mu)$, form a whole set of orthogonal eigenfunctions of corresponding operators of occupation numbers $\hat{N}_{i r}^{\lambda}=\hat{b}_{i r}^{\lambda} \hat{b}_{i_{\lambda}^{r}}^{r}$ with the expectation values 0,1 .

For more detail see (Ter-Kazarian, 1884, 1996, 1999a).

\section{OM: Differential geometric aspect}

The operators $\left\{\hat{\gamma}^{r}\right\}$ are the basis for all operator vectors of tangent section $\hat{\mathbf{T}}_{\Phi_{p}}$ of principle bundle with the base $\hat{G}$ at the point $\boldsymbol{\Phi}_{p}=\left.\boldsymbol{\Phi}(\zeta(t))\right|_{t=0} \in \hat{G}$. The smooth field of tangent operator vector $\hat{\mathbf{A}}(\boldsymbol{\Phi}(\zeta))$ is a class of equivalence of the curves $\mathbf{f}(\boldsymbol{\Phi}(\zeta)), \mathbf{f}(\boldsymbol{\Phi}(\zeta(0)))=\boldsymbol{\Phi}_{p}$. While, the operator differential $\hat{d} A_{p}^{t}$ of the flux $A_{p}^{t}: \hat{G} \rightarrow \hat{G}$ at the point $\boldsymbol{\Phi}_{p}$ with the velocity fields $\hat{\mathbf{A}}(\boldsymbol{\Phi}(\zeta))$ is defined by one parameter group of operator diffeomorphisms given for the curve $\boldsymbol{\Phi}(\zeta(t)): R^{1} \rightarrow \hat{G}$. Provided one has $\boldsymbol{\Phi}(\zeta(0))=\boldsymbol{\Phi}_{p}$ and $\hat{\dot{\boldsymbol{\Phi}}}(\zeta(0))=\hat{\mathbf{A}}_{p} \hat{d} A_{p}^{t}(\mathbf{A})=\left.\frac{\hat{d}}{d t}\right|_{t=0} A^{t}(\boldsymbol{\Phi}(\zeta(t)))=\hat{\mathbf{A}}(\boldsymbol{\Phi}(\zeta))=\hat{\gamma}^{r} A_{p}$, where the $\left\{A_{p}\right\}$ are the components of $\hat{\mathbf{A}}$ in the basis $\left\{\hat{\gamma}^{r}\right\}$. According to eq.(3.2), in holonomic coordinate basis $\hat{\gamma}^{r} \rightarrow\left(\hat{\partial} / \partial \Phi_{r}(\zeta(t))\right)_{p}$ one gets $A_{p}=\left.\frac{\partial \Phi_{r}}{\partial \zeta_{r}} \frac{d \zeta_{r}}{d t}\right|_{p}$. Hence, for any function $f: \mathbf{R}^{n} \rightarrow \mathbf{R}^{n}$ of the ordinary class of functions of $C^{\infty}$ smoothness on $\hat{G}$ one may define an operator differential $<\hat{d} f, \hat{\mathbf{A}}>=\widehat{(A f)}$, by means of smooth reflection $\hat{d} f: \hat{\mathbf{T}}(\hat{G}) \rightarrow \hat{R} \quad\left(\hat{\mathbf{T}}(\hat{G})=\bigcup_{\Phi_{p}} \hat{\mathbf{T}}_{\Phi_{p}}\right)$, where $<\chi\|\hat{d} f, \hat{\mathbf{A}}\| \chi^{0}>=\sum_{\lambda, \mu=1}^{2} \sum_{r^{\lambda \mu}=1}^{N_{\lambda \mu}} \hat{c}^{*}\left(r^{\lambda \mu}\right)<d f, \mathbf{A}>_{r^{\lambda \mu}}=\sum_{\lambda, \mu=1}^{2} \sum_{r^{\lambda \mu}=1}^{N_{\lambda \mu}} \hat{c}^{*}\left(r^{\lambda \mu}\right)(\mathbf{A} f)_{r^{\lambda \mu}}$. In coordinate basis $<d \Phi^{\widehat{\imath}}, \hat{\partial} / \partial \Phi^{j}>=\frac{\partial \Phi^{\widehat{\imath}}}{\partial \Phi^{j}}=\delta_{j}^{\widehat{\imath}}$, provided by $d \Phi^{\widehat{\imath}} \equiv \hat{d} \Phi^{i}$ and

$<\chi\left\|\hat{\delta}_{j}^{i}\right\| \chi^{0}>=\sum_{\lambda, \mu=1}^{2} \sum_{r^{\lambda \mu}=1}^{N_{\lambda \mu}} \hat{c}^{*}\left(r^{\lambda \mu}\right) \delta_{j}^{i}$, where the $i$ and $j$ stand for a set of $\left(\lambda_{i}, \mu_{i}, \alpha_{i}\right)$. The operator tensor $\hat{\mathbf{T}}$ of $\widehat{(n, 0)}$-type at the point $\boldsymbol{\Phi}_{p}$ is a linear function of the space $\hat{\mathbf{T}}_{0}^{n}=\underbrace{\hat{\mathbf{T}}_{\Phi_{p}} \otimes \cdots \otimes \hat{\mathbf{T}}_{\Phi_{p}}}_{n}$, where

the $\otimes$ denotes the tensor product. It enables a correspondence between the element $\left(\hat{\mathbf{A}}_{1}, \ldots, \hat{\mathbf{A}}_{n}\right)$ of $\hat{\mathbf{T}}_{0}^{n}$ and the number $T\left(\hat{\mathbf{A}}_{1}, \ldots, \hat{\mathbf{A}}_{n}\right)$ furnished by linearity. Constructing matrix elements of operator tensors of $\hat{G}$ one produces the Cartan's exterior forms. Whence, the matrix elements of symmetric operator tensors equal zero. The differential operator $n$ form $\left.\hat{\omega}^{n}\right|_{\Phi_{p}}$ at the point $\boldsymbol{\Phi}_{p} \in \hat{G}$ can be defined as the exterior operator $n$ form on tangent operator space $\hat{\mathbf{T}}_{\Phi_{p}}$ of tangent operator vectors $\hat{\mathbf{A}}_{1}, \ldots, \hat{\mathbf{A}}_{n}$. That is, if the $\wedge \hat{\mathbf{T}}_{\Phi_{p}}^{*}(\hat{G})$ means the exterior algebra on $\hat{\mathbf{T}}_{\Phi_{p}}^{*}(\hat{G})$, then operator $n$ form $\left.\hat{\omega}^{n}\right|_{\Phi_{p}}$ is an element of $n$-th degree out of $\wedge \hat{\mathbf{T}}_{\Phi_{p}}^{*}$ depending of the point $\boldsymbol{\Phi}_{p} \in \hat{G}$. Hence $\hat{\omega}^{n}=\left.\bigcup_{\Phi_{p}} \hat{\omega}^{n}\right|_{\Phi_{p}}$. Any differential operator $n$ form of dual operator space $\underbrace{\hat{\mathbf{T}}_{\Phi_{p}}^{*} \otimes \cdots \otimes \hat{\mathbf{T}}_{\Phi_{p}}^{*}}_{n}$ may be written $\hat{\omega}^{n}=$ $\sum_{i_{1}<\cdots<i_{n}} \alpha_{i_{1} \cdots i_{n}}(\Phi) d \Phi^{\widehat{\imath}_{1}} \wedge \cdots \wedge d \Phi^{\widehat{\imath}_{n}}$, provided by the smooth differentiable functions $\alpha_{i_{1} \cdots i_{n}}(\Phi) \in C^{\infty}$ and basis $d \Phi^{\widehat{\imath}_{1}} \wedge \cdots \wedge d \Phi^{\widehat{\imath}_{n}}=\sum_{\sigma \in S_{n}} \operatorname{sgn}(\sigma) \gamma^{\sigma\left(\widehat{\imath}_{1}\right.} \otimes \cdots \otimes \gamma^{\left.\widehat{\imath}_{n}\right)}$. The linear operator form of 1 degree $\hat{\omega}^{1}$ is a linear operator valued function on $\hat{\mathbf{T}}_{\Phi_{p}}$, namely $\hat{\omega}^{1}\left(\hat{\mathbf{A}}_{p}\right): \hat{\mathbf{T}}_{\Phi_{p}} \rightarrow \hat{R}$, where $\hat{\mathbf{A}}_{p} \in \hat{\mathbf{T}}_{\Phi_{p}}$, and the operator $\hat{\omega}^{1}(\hat{\mathbf{A}})=<\hat{\omega}^{1}, \mathbf{A}>\in \hat{R}$ corresponds to $\hat{\mathbf{A}}_{p}$ at the point $\boldsymbol{\Phi}_{p}$, provided, according to eq.(A.1.25), with $<\chi\left\|\hat{\omega}^{1}\right\| \chi^{0}>=\sum_{\lambda, \mu=1}^{2} \sum_{r^{\lambda \mu}=1}^{N_{\lambda \mu}} \hat{c}^{*}\left(r^{\lambda \mu}\right) \omega_{r^{\lambda \mu}}^{1}$, where $\omega_{r^{\lambda \mu}}^{1}=e_{r^{\lambda \mu}}^{(\lambda, \mu, \alpha)} \omega_{(\lambda, \mu, \alpha)}^{r^{\lambda \mu}}$, the $<\omega_{r^{\lambda \mu}}^{1}, \mathbf{A}>=\omega_{r^{\lambda \mu}}^{1}(\mathbf{A})$ is a linear form on $\mathbf{T}_{p}$, and $\hat{\omega}^{1}\left(\lambda_{1} \hat{\mathbf{A}}_{1}+\lambda_{2} \hat{\mathbf{A}}_{2}\right)=\lambda_{1} \hat{\omega}^{1}\left(\hat{\mathbf{A}}_{1}\right)+\lambda_{2} \hat{\omega}^{1}\left(\hat{\mathbf{A}}_{2}\right), \quad \forall \lambda_{1}, \lambda_{2} \in R, \quad \hat{\mathbf{A}}_{1}, \hat{\mathbf{A}}_{2} \in \hat{\mathbf{T}}_{\Phi_{p}}$. The set of all linear operator forms defined at the point $\boldsymbol{\Phi}_{p}$ fill up the operator vector space $\hat{\mathbf{T}}_{\Phi_{p}}^{*}$ dual to $\hat{\mathbf{T}}_{\Phi_{p}}$. While, the $\left\{\hat{\gamma}_{r}\right\}$ serves as a basis for them. The operator $n$ form is defined as the exterior product of operator 1 forms. Here as well as for the rest of this section we abbreviate the set of indices $\left(\lambda_{i}, \mu_{i}, \alpha_{i}\right)$ by the single symbol $i$. Let the $\hat{\mathcal{D}}_{1}$ and $\hat{\mathcal{D}}_{2}$ are two compact convex parallelepipeds in oriented $n$ dimensional operator space $\hat{\mathbf{R}}^{n}$ and the $f: \hat{\mathcal{D}}_{1} \rightarrow \hat{\mathcal{D}}_{2}$ is differentiable reflection of interior of $\hat{\mathcal{D}}_{1}$ into $\hat{\mathcal{D}}_{2}$ retaining an orientation, namely for any function $\varphi \in C^{\infty}$ defined on $\hat{\mathcal{D}}_{2}$ it holds $\varphi \circ f \in C^{\infty}$ and $f^{*} \varphi\left(\boldsymbol{\Phi}_{p}\right)=\varphi\left(f\left(\boldsymbol{\Phi}_{p}\right)\right)$, where $f^{*}$ is an image of function $\varphi\left(f\left(\boldsymbol{\Phi}_{p}\right)\right)$ on $\hat{\mathcal{D}}_{1}$ at the point $\boldsymbol{\Phi}_{p}$. Hence, the function $f$ induces a linear reflection $\hat{d} f: \hat{\mathbf{T}}\left(\hat{\mathcal{D}}_{1}\right) \rightarrow \hat{\mathbf{T}}\left(\hat{\mathcal{D}}_{2}\right)$ as an operator differential of $f$ implying $\hat{d} f\left(\hat{\mathbf{A}}_{p}\right) \varphi=\hat{\mathbf{A}}_{p}(\varphi \circ f)$ for any operator vector $\hat{\mathbf{A}}_{p} \in \hat{\mathbf{T}}_{\Phi_{p}}$ and for any function $\varphi \in C^{\infty}$ defined in the neighbourhood of $\boldsymbol{\Phi}_{p}^{\prime}=f\left(\boldsymbol{\Phi}_{p}\right)$. If the function $f$ is 
given in the form $\Phi^{\prime i}=\Phi^{\prime i}\left(\Phi_{p}\right)$ and $\hat{\mathbf{A}}_{p}=\left(A^{i} \hat{\partial} / \partial \Phi^{i}\right)_{p}$, then in terms of local coordinates one gets $(\hat{d} f) \hat{\mathbf{A}}_{p}=A^{i}\left(\frac{\partial \Phi^{\prime j}}{\partial \Phi^{i}}\right)_{p}\left(\frac{\hat{\partial}}{\partial \Phi^{\prime j}}\right)_{p^{\prime}}$. So, if $f_{1}: \hat{\mathcal{D}}_{1} \rightarrow \hat{\mathcal{D}}_{2}$ and $f_{2}: \hat{\mathcal{D}}_{2} \rightarrow \hat{\mathcal{D}}_{3}$ then $\hat{d}\left(f_{2} \circ f_{1}\right)=\hat{d} f_{2} \circ \hat{d} f_{1}$. For any differential operator $n$ form $\hat{\omega}^{n}$ on $\hat{\mathcal{D}}_{2}$ the reflection $f$ induces the operator $n$ form $\hat{f}^{*} \hat{\omega}^{n}$ on $\left.\hat{\mathcal{D}}_{1}\left(\hat{f}^{*} \hat{\omega}^{n}\right)\left(\hat{\mathbf{A}}_{1}, \ldots, \hat{\mathbf{A}}_{n}\right)\right|_{\Phi_{p}}=\left.\hat{f}_{*} \hat{\omega}^{n}\left(\hat{f}_{*} \hat{\mathbf{A}}_{1}, \ldots, \hat{f}_{*} \hat{\mathbf{A}}_{n}\right)\right|_{f\left(\Phi_{p}\right)}$. We may consider the integration of operator $n$ form implying $\int_{\hat{\mathcal{D}}_{1}} \hat{f}^{*} \hat{\omega}^{n}=\int_{\hat{\mathcal{D}}_{2}} \hat{\omega}^{n}$. In general, let $\hat{\mathcal{D}}_{1}$ is the limited convex $n$ dimensional parallelepiped in the $n$ dimensional operator space $\hat{\mathbf{R}}^{n}$. One defines the $n$ dimensional $i$-th piece of integration path $\hat{\sigma}^{i}$ in $\hat{G}$ as $\hat{\sigma}^{i}=\left(\hat{\mathcal{D}}_{i}, f_{i}, O r_{i}\right)$, where $\hat{\mathcal{D}}_{i} \in \hat{\mathbf{R}}^{n}, \quad f_{i}: \hat{\mathcal{D}}_{i} \rightarrow \hat{G}$ and the $O r_{i}$ is an orientation of $\hat{\mathbf{R}}^{n}$. Then, the integral over the operator $n$ form $\hat{\omega}^{n}$ along the operator $n$ dimensional chain $\hat{c}_{n}=\sum m_{i} \hat{\sigma}^{i}$ may be written $\int_{\hat{c}_{n}} \hat{\omega}^{n}=\sum m_{i} \int_{\hat{\sigma}^{i}} \hat{\omega}^{n}=\sum m_{i} \int_{\hat{\mathcal{D}}_{i}} \hat{f}^{*} \hat{\omega}^{n}$, where the $m_{i}$ is a multiple number. Next, we may apply the analog of exterior differentiation. We define the operator $(n+1)$ form $\hat{d} \hat{\omega}^{n}$ on $(n+1)$ operator vectors $\hat{\mathbf{A}}_{1}, \ldots, \hat{\mathbf{A}}_{n+1} \in \hat{\mathbf{T}}_{\Phi_{p}}$ by considering diffeomorphic reflection $f$ of the neighbourhood of the point 0 in $\hat{\mathbf{R}}^{n}$ into neighbourhood of the point $\boldsymbol{\Phi}_{p}$ in $\hat{G}$. The prototypes of operator vectors $\hat{\mathbf{A}}_{1}, \ldots, \hat{\mathbf{A}}_{n+1} \in \hat{\mathbf{T}}_{\Phi_{p}}(\hat{G})$ at the operator differential of $f$ belong to tangent operator space $\hat{\mathbf{R}}^{n}$ in 0 . Namely, the prototypes are the operator vectors $\hat{\xi}_{1}, \ldots, \hat{\xi}_{n+1} \in \hat{\mathbf{R}}^{n}$. Let $f$ reflects the parallelepiped $\hat{\boldsymbol{\Pi}}^{*}$, stretched over the $\hat{\xi}_{1}, \ldots, \hat{\xi}_{n+1}$, onto the $(n+1)$ dimensional piece $\hat{\boldsymbol{\Pi}}$ on the $\hat{G}$. While the border of the $n$ dimensional chain $\partial \hat{\mathbf{\Pi}}$ in $\hat{\mathbf{R}}^{n+1}$ defined as follows: the pieces $\hat{\sigma}^{i}$ of the chain $\partial \hat{\mathbf{\Pi}}$ are $n$ dimensional facets $\partial \hat{\boldsymbol{\Pi}}_{i}$ of parallelepiped $\partial \hat{\boldsymbol{\Pi}}$ with the reflections embedding the facets into $\hat{\mathbf{R}}^{n+1}: \quad f_{i}: \hat{\mathbf{\Pi}}_{i} \rightarrow \hat{\mathbf{R}}^{n+1}$, and the orientations $O r_{i}$ has defined as $\partial \hat{\mathbf{\Pi}}=\sum \hat{\sigma}^{i}, \quad \hat{\sigma}^{i}=\left(\hat{\boldsymbol{\Pi}}_{i}, f_{i}, O r_{i}\right)$ Considering the curvilinear parallelepiped $F\left(\hat{\mathbf{A}}_{1}, \ldots, \hat{\mathbf{A}}_{n}\right)=\int_{\partial \hat{\boldsymbol{\Pi}}} \hat{\omega}^{n}$, one may state that the unique operator of the $(n+1)$-form $\hat{\Omega}$ exists on $\hat{\mathbf{T}}_{\Phi_{p}}$, which is the principle $(n+1)$ linear part in 0 of integral over the border of $F\left(\hat{\mathbf{A}}_{1}, \ldots, \hat{\mathbf{A}}_{n}\right)$, namely $F\left(\varepsilon \hat{\mathbf{A}}_{1}, \ldots, \varepsilon \hat{\mathbf{A}}_{n}\right)=\varepsilon^{n+1} \hat{\Omega}\left(\hat{\mathbf{A}}_{1}, \ldots, \hat{\mathbf{A}}_{n+1}\right)+O\left(\varepsilon^{n+1}\right)$, where $\hat{\Omega}$ is independent of choice of the coordinates used in definition of $F$. The prove of it is the same to those of similar one given in the differential geometry.

At this point we cut short our discussion, and refer the interested reader to original papers (TerKazarian, 1884, 1996, 1999a) for more detailed justification of some of the procedures and complete exposition of a lengthy mathematical apparatus of OM.

\section{Primordial structures and link establishing processes}

We have chosen a simple setting and considered the primordial structures designed to possess certain physical properties satisfying the stated general rules. These structures are thought to be the substance out of which the geometry and particles are made.

\subsection{The Regular Primordial Structures}

We distinguish $\eta$ - and $u$-types primordial structures involved in the linkage establishing processes occurring between the structures of different types. The $\eta$-type structure may accept the linkage only from $u$-type structure, which is described by the link function $\psi_{\eta}(\eta)$ belonging to the ordinary class of functions of $C^{\infty}$ smoothness, where $\eta=e_{\eta(\lambda \alpha)} \eta^{(\lambda \alpha)},(\lambda= \pm ; \alpha=1,2,3), \eta$ is the link coordinate. Respectively the $u$-type structure may accept the linkage only from $\eta$-type structure described by the link function $\psi_{u}(u)$ (u-channel, $u=e_{u} u$ ), where $\psi_{\eta}{ }^{( \pm \alpha)}\left(\eta, p_{\eta}\right)=\eta^{( \pm \alpha)} \psi_{\eta}{ }^{ \pm}\left(\eta, p_{\eta}\right)$, $\psi_{u}{ }^{( \pm \alpha)}\left(u, p_{u}\right)=u^{( \pm \alpha)} \psi_{u}^{ \pm}\left(u, p_{u}\right)$, a bispinor ${\psi_{i}}^{ \pm}$is the invariant state wave function of positive or negative frequencies, $p_{i}$ is the corresponding link momentum. Thus, a primordial structure can be considered as a fermion. A simplest system made of two structures of different types becomes stable only due to the stable linkage $\left|p_{\eta}\right|=\left(p_{\eta}{ }^{(\lambda \alpha)}, p_{\eta(\lambda \alpha)}\right)^{1 / 2}=\left|p_{u}\right|=\left(p_{u}{ }^{(\lambda \alpha)}, p_{u(\lambda \alpha)}\right)^{1 / 2}$. Otherwise they are unstable. There is not any restriction on the number of primordial structures of both types involved in the link establishing processes simultaneously. Only, in the stable system the link stability condition must be held for each linkage separately. Suppose that persistent processes of creation and annihilation of the primordial structures proceed in different states $s, s^{\prime}, s^{\prime \prime}, \ldots$ The "creation" of structure in the given state $(s)$ is due to its transition to this state from other states $\left(s^{\prime}, s^{\prime \prime}, \ldots\right)$, while the "annihilation" means a vice versa. Satisfying the stability condition the primordial structures from arbitrary states 
can establish a stable linkage. Among the states $\left(s, s^{\prime}, s^{\prime \prime}, \ldots\right)$ there is a lowest one $\left(s_{0}\right)$, in which all structures are regular, i.e., they are in free (pure) state and described by the plane wave functions $\psi_{\eta}{ }^{ \pm}\left(\eta_{f}, p_{\eta}\right)$ or $\psi_{u}^{ \pm}\left(u_{f}, p_{u}\right)$ defined respectively on flat manifolds $G_{\eta}$ and $G_{u}$. The index (f) specifies the points of corresponding flat manifolds $\eta_{f} \in G_{\eta}, u_{f} \in G_{u}$. Note that the processes of creation and annihilation of regular structures in lowest state are described by the OM formalism given above.

\subsection{Distorted primordial structures}

In all higher states the primordial structures are distorted (interaction states) and described by distorted link functions defined on distorted manifolds $\widetilde{G_{\eta}}$ and $\widetilde{G_{u}}$. The distortion $G \rightarrow \widetilde{G}$ with hidden Abelian local group $G=U^{l o c}(1)=S O^{l o c}(2)$ and one dimensional trivial algebra $\hat{g}=R^{1}$ is considered in (Ter-Kazarian, 2010). Within that scheme the basis $e^{f}$ undergoes distortion transformation $e(\theta)=D(\theta) e^{f}$. The matrix $D(\theta)$ is in the form $D(\theta)=C \otimes R(\theta)$, where $O_{(\lambda \alpha)}=C_{(\lambda \alpha)}^{\tau} O_{\tau}$ and $\sigma_{(\lambda \alpha)}(\theta)=R_{(\lambda \alpha)}^{\beta}(\theta) \sigma_{\beta}$. Here $R(\theta)$ is the matrix of the group $S O(3)$ of ordinary rotations of the planes involving two arbitrary basis vectors of the spaces $R_{ \pm}^{3}$ around the orthogonal third axes $( \pm k)$ through the angle $\left(\theta_{ \pm k}\right)$. The relation between the wave functions of distorted and regular structures reads $\psi_{u}{ }^{\lambda}\left(\theta_{+k}\right)=f_{(+)}\left(\theta_{+k}\right) \psi_{u}{ }^{\lambda}, \psi_{u \lambda}\left(\theta_{-k}\right)=\psi_{u \lambda} f_{(-)}\left(\theta_{-k}\right)$, where $\psi_{u}{ }^{\lambda}\left(\psi_{u \lambda}\right)$ is the plane wave function of regular ordinary structure (antistructure). Next, we supplement the previous assumptions given in subsec.2.3 by the new one that now the $\eta$-type (fundamental) regular structure can not directly form a stable system with the regular $u$-type (ordinary) structures. Instead of it the $\eta$-type regular structure forms a stable system with the infinite number of distorted ordinary structures, where the link stability condition held for each linkage separately. Such structures take part in realization of flat manifold $G$. We employ the wave packets constructed by superposition of these functions furnished by generalized operators of creation and annihilation as the expansion coefficients. Geometry realization condition now should be satisfied for each ordinary structure in terms of $G_{u F}{ }_{F}^{\theta}(0)=\lim _{\theta_{+} \rightarrow \theta_{-}} G_{u F}{ }_{i}^{\theta}\left(\theta_{+}-\theta_{-}\right)=G_{\eta F}(0)=\lim _{\eta_{f}^{\prime} \rightarrow \eta_{f}} G_{\eta F}\left(\eta_{f}^{\prime}-\eta_{f}\right)$. Then $\sum_{k} \psi_{u}\left(\theta_{+k}\right) \bar{\psi}_{u}\left(\theta_{-k}\right)=\sum_{k} \psi_{u}{ }^{\prime}\left(\theta_{+k}^{\prime}\right) \bar{\psi}_{u}{ }^{\prime}\left(\theta_{-k}^{\prime}\right)=\cdots=i n v$. Namely, the distorted ordinary structures emerge in geometry only in permissible combinations forming a stable system. Below, in simplified schematic way we exploit the background of the known colour confinement and gauge principles. Naive version of such construction still should be considered as a preliminary one, which will be further elaborated to introduce basis for subquarks.

\subsection{Quarks and colour confinement}

At the very first to avoid irrelevant complications, here, for illustrative purposes, we will attempt to introduce temporarily skeletonized 'quark' and 'antiquark' fields emerged in confined phase in the simplified geometry with the one-u channel given in the previous subsections. The complete picture of such a dynamics is beyond the scope of this subsection, but some relevant discussions on this subject will also be presented. We may think of the function $\psi_{u}{ }^{\lambda}\left(\theta_{+k}\right)$ at fixed $(k)$ as being $u$-component of bispinor field of "quark" $q_{k}$, and of $\bar{\psi}_{u \lambda}\left(\theta_{-k}\right)$ - an $u$-component of conjugated bispinor field of "antiquark" $\bar{q}_{k}$. The index $(k)$ refers to colour degree of freedom in the case of rotations through the angles $\theta_{+k}$ and anticolour degree of freedom in the case of $\theta_{-k}$. The $\eta$-components of quark fields are plane waves. There are exactly three colours. The rotation through the angle $\theta_{+k}$ yields a total quark field $q_{k}(\theta)=\Psi\left(\theta_{+k}\right)=\psi_{\eta}{ }^{0} \psi_{u}\left(\theta_{+k}\right)$ where $\psi_{\eta}{ }^{0}$ is a plane wave defined on $G_{\eta}$. This allows an other interpretation of quarks, which is absolutely equivalent to the former one and will be widely used throughout this article, i.e., $q_{k}(\theta)=\psi_{\eta}{ }^{0} q_{u k}(\theta)=q_{\eta k}(\theta) \psi_{u}{ }^{0}, q_{\eta k}(\theta) \equiv f_{(+)}\left(\theta_{+k}\right) \psi_{\eta}{ }^{0}$, where $\psi_{u}{ }^{0}$ is a plane wave, $q_{u k}(\theta)$ and $q_{\eta k}(\theta)$ may be considered as the quark fields with the same quantum numbers defined respectively on flat manifolds $G_{u}$ and $G_{\eta}$. Making use of the rules stated one may readily return to Minkowski space $G_{\eta} \rightarrow M_{4}$. In the sequel, a conventional quark fields defined on $M_{4}$ will be ensued $q_{\eta k}(\theta) \rightarrow q_{k}(x), x \in M_{4}$. They imply $\sum_{k} q_{k p} \bar{q}_{k p}=\sum_{k} q^{\prime}{ }_{k p} \bar{q}^{\prime}{ }_{k p}=\cdots=i n v$. It utilizes the idea of colour confinement principle: the quarks emerge in the geometry only in special combinations of colour singlets. Only two colour singlets are available $(q \bar{q})=\frac{1}{\sqrt{3}} \delta_{k k^{\prime}} \hat{q}_{k} \overline{\hat{q}}_{k^{\prime}}=i n v$, $(q q q)=\frac{1}{\sqrt{6}} \varepsilon_{k l m} \hat{q}_{k} \hat{q}_{l} \hat{q}_{m}=i n v$. 


\subsection{Gauge principle-internal symmetries}

Each regular structure in the lowest state can be regarded as a result of transition from an arbitrary state, in which they assumed to be distorted. Hence, the following transformations may be implemented upon distorted ordinary structures $\psi_{u}{ }^{\prime \lambda}\left(\theta_{+l}^{\prime}\right)=f_{l k}^{(+)} \psi_{u}{ }^{\lambda}\left(\theta_{+k}\right)=f\left(\theta_{+l}^{\prime}, \theta_{-k}\right) \psi_{u}{ }^{\lambda}\left(\theta_{+k}\right)$, $f\left(\theta_{+l}^{\prime}, \theta_{-k}\right)=f_{(+)}\left(\theta_{l}^{\prime}\right) f_{(-)}\left(\theta_{k}\right)$. The transformation functions are the operators in the space of internal degrees of freedom labeled by $( \pm k)$ corresponding to distortion rotations around the axes $( \pm k)$ by the angles $\theta_{ \pm k}$. We make proposition that the distortion rotations are incompatible, namely the transformation operators $f_{l k}^{( \pm)}$obey the incompatibility relations $f_{l k}^{(+)} f_{c d}^{(+)}-f_{l d}^{(+)} f_{c k}^{(+)}=\left\|f^{(+)}\right\| \varepsilon_{l c m} \varepsilon_{k d n} f_{n m}^{(-)}$, $f_{k l}^{(-)} f_{d c}^{(-)}-f_{d l}^{(-)} f_{k c}^{(-)}=\left\|f^{(-)}\right\| \varepsilon_{l c m} \varepsilon_{k d n} f_{m n}^{(+)}$, where $l, k, c, d, m, n=1,2,3$. This relations hold in general for both local and global rotations. Then one gets the transformations implemented upon the quark field, which in matrix notation take the form $q^{\prime}(\zeta)=U(\theta(\zeta)) q(\zeta), \overline{q^{\prime}}(\zeta)=\bar{q}(\zeta) U^{+}(\theta(\zeta))$, where $q=\left\{q_{k}\right\}, \quad U(\theta)=\left\{f_{l k}^{(+)}\right\}$. Due to the incompatibility commutation relations the transformation matrices $\{U\}$ generate the unitary groups of internal symmetries $U(1), S U(2), S U(3)$ corresponding to one-, two- and three-dimensional rotations through the angles $\theta_{ \pm k}$, while an action of physical system must be invariant under such transformations (gauge principle).

\section{Operator Multimanifold $\hat{G}_{N}$}

In the second part, we generalized the knowledge gained in outlined mathematical framework via the concept of operator multimanifold (OMM), which yields the multiworld (MW)-geometry. The latter involves the spacetime continuum and internal worlds of the given number.

\subsection{Operator Vector and Covector Fields}

The OM formalism of $\hat{G}=\hat{G}_{\eta} \oplus \hat{G}_{u}$ is built up by assuming an existence only of ordinary primordial structures of one sort (one u-channel). Being confronted by our major goal to develop the microscopic approach to field theory based on multiworld geometry, henceforth we generalize the OM formalism via the concept of the OMM. Then, instead of one sort of ordinary structures we are going to deal with different species of ordinary structures. But before proceeding further and to enlarge the previous model it is profitable to assume an existence of infinite number of ${ }^{i} u$-type ordinary structures of different species $i=1,2, \ldots, N$ (multi-u channel). These structures will be specified by the superscript $i$ to the left. This hypothesis, as it will be seen in the subsequent part II, leads to the substantial progress of understanding of the properties of particles. At the very outset we consider the processes of creation and annihilation of regular structures of $\eta$ - and ${ }^{i} u$-types in the lowest state $\left(s_{0}\right)$. The general rules stated in subsec 2.1 regarding to this change apply a substitution of operator basis pseudo vectors and covectors by a new ones $(i=1,2, \ldots, N):{ }^{i} \hat{O}_{\lambda, \mu}^{r_{1} r_{2}}={ }^{i} \hat{O}_{\lambda}^{r_{1}} \otimes{ }^{i} \hat{O}_{\mu}^{r_{2}} \equiv{ }^{i} \hat{O}_{\lambda, \mu}^{r}={ }^{i} O_{\lambda, \mu}^{r}\left(\alpha_{\lambda} \otimes \alpha_{\mu}\right)$, provided by $r \equiv\left(r_{1}, r_{2}\right)$ and ${ }^{i} O_{1,1}^{r}=\frac{1}{\sqrt{2}}\left(\nu_{i} O_{\eta_{+}}^{r}+{ }^{i} O_{u_{+}}^{r}\right),{ }^{i} O_{2,1}^{r}=\frac{1}{\sqrt{2}}\left(\nu_{i} O_{\eta_{+}}^{r}-{ }^{i} O_{u+}^{r}\right),{ }^{r} O_{1,2}^{r}=\frac{1}{\sqrt{2}}\left(\nu_{i} O_{\eta_{-}}^{r}+{ }^{i} O_{u-}^{r}\right)$, ${ }^{i} O_{2,2}^{r}=\frac{1}{\sqrt{2}}\left(\nu_{i} O_{\eta-}^{r}-{ }^{i} O_{u-}^{r}\right)$, where $\left\langle\nu_{i}, \nu_{j}>=\delta_{i j},\left\langle{ }^{i} O_{u \lambda}^{r},{ }^{j} O_{u \tau}{ }_{r^{\prime}}^{r^{\prime}}>=-\delta_{i j} \delta_{r r^{\prime}}{ }^{*} \delta_{\lambda \tau},\left\langle O_{\eta \lambda}{ }^{r},{ }^{i} O_{u_{\tau}}^{r^{\prime}}>=0\right.\right.\right.$. We consider then the operators ${ }^{i} \hat{\gamma}_{(\lambda, \mu, \alpha)}^{r}={ }^{i} \hat{O}_{\lambda, \mu}^{r_{1} r_{2}} \otimes \hat{\sigma}_{\alpha}^{r_{3}}$. and calculate nonzero matrix elements $<\lambda, \mu\left|{ }^{i} \hat{\gamma}_{(\tau, \nu, \alpha)}^{r}\right| \tau, \nu>={ }^{*} \delta_{\lambda \tau}{ }^{*} \delta_{\mu, \nu}{ }^{i} e_{(\tau, \nu, \alpha)}^{r}$, where ${ }^{i} e_{(\lambda, \mu, \alpha)}^{r}={ }^{i} O_{\lambda, \mu}^{r} \otimes \sigma_{\alpha}$. The operators $\left\{{ }^{i} \hat{\gamma}^{r}\right\}$ are the basis for all the operator vectors $\hat{\Phi}(\zeta)={ }^{i} \hat{\gamma}^{r}{ }^{i} \Phi_{r}(\zeta)$ of tangent section of principle bundle with the base of operator multimanifold $\hat{G}_{N}=\left(\sum_{i}^{N} \oplus^{*} \hat{R}_{i}^{4}\right) \otimes \hat{R}^{3}$. Here ${ }^{*} \hat{R}_{i}^{4}$ is the $2 \times 2$ dimensional linear pseudo operator space, with the set of the linear unit operator pseudo vectors as the basis of tangent vector section, and $\hat{R}^{3}$ is the three dimensional real linear operator space with the basis consisted of the ordinary unit operator vectors $\left\{\hat{\sigma}_{\alpha}^{r}\right\}$. The $\hat{G}_{N}$ decomposes as follows: $\hat{G}_{N}=\hat{G}_{\eta} \oplus \hat{G}_{u_{1}} \oplus \cdots \oplus \hat{G}_{u_{N}}$, where $\hat{G}_{u_{i}}$ is the six dimensional operator manifold of the given species $(i)$ with the basis $\left\{\hat{\gamma}_{u(\lambda \alpha)}^{r}={ }^{i} \hat{O}_{u_{\lambda}^{r}}^{r} \otimes \hat{\sigma}_{\alpha}^{r}\right\}$. The expansions of operator vectors and covectors are written $\hat{\Psi}_{\eta}=\hat{\gamma}_{\eta}^{r} \Psi_{\eta r}$, $\hat{\Psi}_{u}={ }^{i} \hat{\gamma}_{u}{ }^{r} \Psi_{u r}, \overline{\hat{\Psi}}_{\eta}=\hat{\gamma}_{\eta r} \Psi_{\eta}{ }^{r}, \overline{\hat{\Psi}}_{u}={ }^{i} \hat{\gamma}_{u r}{ }^{i} \Psi_{u}{ }^{r}$, where the components $\Psi_{\eta r}(\eta)$ and ${ }^{i} \Psi_{u r}(u)$ are respectively the link functions of $\eta$-type and ${ }^{i} u$-type structures. 


\subsection{Realization of the Multimanifold $G_{N}$}

Now, we consider the special system of the regular structures, which is made of one fundamental structure of $\eta$-type and infinite number of ${ }^{i} u$-type ordinary structures of different species $(i=1, \ldots, N)$. The primordial structures establish the stable linkage to form the stable system $p^{2}=p_{\eta}^{2}-\sum_{i=1}^{N} p_{u_{i}}^{2}=0$. The free field defined on the multimanifold $G_{N}=G_{\eta} \oplus G_{u_{1}} \oplus \cdots \oplus G_{u_{N}}$ is written $\Psi=\Psi_{\eta}(\eta) \Psi_{u}(u)$, $\Psi_{u}(u)=\Psi_{u_{1}}\left(u_{1}\right) \cdots \Psi_{u_{N}}\left(u_{N}\right)$, where $\Psi_{u_{i}}$ is the bispinor defined on the internal manifold $G_{u_{i}}$. On analogy to above, we make use of localized wave packets by means of superposition of plane wave solutions furnished by creation and annihilation operators in agreement with Pauli's principle. Straightforward calculations now give $\sum_{\lambda= \pm}<\chi_{\lambda}|\hat{\Phi}(\zeta) \overline{\hat{\Phi}}(\zeta)| \chi_{\lambda}>=\sum_{\lambda= \pm}<\chi_{\lambda}|\overline{\hat{\Phi}}(\zeta) \hat{\Phi}(\zeta)| \chi_{\lambda}>=-i \zeta^{2} G_{\zeta}(0)=$ $-i\left(\eta^{2} G_{\eta}(0)-\sum_{i=1}^{N} u_{i}{ }^{2} G_{u_{i}}(0)\right)$. Along the same line the realization of the multimanifold stems from the condition as alluded to above. Let denote $u^{2} G_{u}(0) \equiv \lim _{u_{i} \rightarrow u_{i}^{\prime}} \sum_{i=1}^{N}\left(u_{i} u_{i}^{\prime}\right) G_{u_{i}}\left(u_{i}-u_{i}^{\prime}\right)$ and consider a stable system. Hence $G_{u F}(0)=G_{\eta F}(0)=G_{\zeta F}(0)$, where $G_{\eta F}, G_{u F}$ and $G_{\zeta F}$ are the causal Green's functions of the $\eta-, u-$ and $\zeta$-type structures, and $m \equiv\left|p_{u}\right|=\left(\sum_{i=1}^{N} p_{u_{i}}{ }^{2}\right)^{1 / 2}=\left|p_{\eta}\right|$. In the aftermath, the length of each vector $\zeta={ }^{i} e{ }^{i} \zeta \in G_{N}$ should be equaled zero (subsec.2.2) $\zeta^{2}=\eta^{2}-u^{2}=$ $\eta^{2}-\sum_{i=1}^{N}\left(u_{i}^{G}\right)^{2}=0$, where use is made of $\left(u_{i}^{G}\right)^{2} \equiv u_{i}^{2} \lim _{u_{i} \rightarrow u_{i}^{\prime}} G_{u_{i} F}\left(u_{i}-u_{i}^{\prime}\right) / G_{\eta F}\left(\eta-\eta^{\prime}\right)$ and $\eta \rightarrow \eta^{\prime}$

$u_{i}^{G}={ }^{i} \hat{e}_{u(\lambda, \alpha)} u_{i}^{G(\lambda, \alpha)}$. Thus, the multimanifold $G_{N}$ comes into being, which decomposes as follows: $G_{N}=G_{\eta} \oplus G_{u_{1}} \oplus \cdots$ Meanwhile, the Minkowski flat space $M_{4}$ stems from the flat submanifold $G_{\eta}$ , in which the line element turned out to be invariant. That is, the principle of relativity comes into being with the $M_{4}$ ensued from the MW geometry $G_{N}$. In the following we shall use a notion of the $i$-th internal world for the submanifold $G_{u_{i}}$.

\subsection{The subquark algebra and supercharges}

The following transformations are implemented upon the subquarks (antisubquarks) on the given (i) internal world: $q_{l}^{\prime}=f_{l k}^{(+)} q_{k}, \bar{q}_{l}^{\prime}=\bar{q}_{k} f_{k l}^{(-)}$, where as well for the next section we left implicit the MWsuperscript $(i)$ to the left. Then, the following composition rules hold for the transformation functions $f_{l k}^{(+)}=f_{l} \circ f_{k}^{-1}, f_{l k}^{(-)}=\bar{f}_{l} \circ \bar{f}_{k}^{-1},\left(f_{l} \circ f_{k}^{-1}\right)\left(f_{c} \circ f_{d}^{-1}\right)=\left(f_{l} f_{c}\right) \circ\left(f_{k}^{-1} f_{d}^{-1}\right)$, where $l, k, c, d=1,2,3$, the transformation functions $f_{k} \equiv f_{(+)}\left(\theta_{+k}\right)$ and $\bar{f}_{l} \equiv f_{(-)}\left(\theta_{-k}\right)$ are the operators in the space of internal degrees of freedom labeled by the subcolour index $( \pm k)$ such that the rotation through the angle $\theta_{ \pm k}$ yields the subquark (antisubquark) field $q_{k}=f_{k} q_{0}, \bar{q}_{k}=\bar{q}_{0} \bar{f}_{k}$. The incompatibility commutation relations with the composition rule lead to the following commutation relations $\left[f_{l}, f_{k}\right]=\epsilon_{l k m} \bar{f}_{m}$. Whence, the subquarks imply $\left[q_{l}, q_{k}\right]=Q_{0} \epsilon_{l k m} \bar{q}_{m}, Q_{0} \equiv q_{0}^{2} / \bar{q}_{0}$. The symmetries of the $C-(C \equiv s, c, b, t)$ and $Q$ - worlds are assumed to be respectively global and local unitary $\operatorname{diag}(S U(3))$ symmetries, for which $q_{l} q_{k}=Q_{0}^{\prime} \epsilon_{l k m} \bar{q}_{m}$, while for the $W$-world with the unified symmetry $S U(2)_{L} \otimes U(1)$ it reduced to $\left[q_{1 L}, q_{2 L}\right]=Q_{0} \bar{q}_{2 R},\left[q_{2 L}, q_{2 R}\right]=Q_{0} \bar{q}_{1 L},\left[q_{2 R}, q_{1 L}\right]=Q_{0} \bar{q}_{2 L}$, where the subcolour singlets are $Q_{2 R},\left[q_{1 L}, q_{2 L}\right]$ and $(q \bar{q})$. Hence, for the electron and corresponding neutrino one gets $\left[\nu_{L}, e_{L}\right]=Q_{0} \bar{e}_{R}$, $\left[e_{L}, e_{R}\right]=Q_{0} \bar{\nu}_{L},\left[e_{R}, \nu_{L}\right]=Q_{0} \bar{e}_{L}$. The important relation between the fermionic $(F)$ and bosonic $(B)$ subcolour singlets reads $(q q q) \equiv \frac{1}{\sqrt{6}} \epsilon_{l k m} q_{l} q_{k} q_{m}=Q_{0 u}(q \bar{q}) \equiv Q_{0 u} \frac{1}{\sqrt{3}}\left(q_{k} \bar{q}_{k}\right), \quad F \rightarrow Q_{0 u} B$, and vice versa, where $F \equiv(q q q), \quad B \equiv(q \bar{q}), \quad Q_{0 u} \equiv \frac{1}{\sqrt{2}} Q_{0}$. It means that considered physical system must respect the invariance under a symmetry group of the fermion-boson transformations occurred in the internal worlds. The latter is known as a "supersymmetry". It is why the basis vectors in the Hilbert space $\mathcal{H}$ have taken to be in the form $\left|n_{B} n_{F}\right\rangle$, where the boson and fermion occupation numbers respectively are $n_{B}=1,2, \ldots, \infty$ and $n_{F}=0,1$. It is convenient, then, to describe such a quantum mechanical system as the spin- $1 / 2$ like supersymmetric particle with mass $m=\left(\frac{\hbar}{Q_{0 u}}\right)^{2}$ moving along the one-dimensional Euclidean line $\mathcal{R}$. Therefore, one introduces a generalized bosonic operator $b$ and a fermionic operator $f$ acting on the Hilbert space $\mathcal{H}=L^{2}(\mathcal{R}) \otimes \mathcal{C}^{2}$ : $b: L^{2}(\mathcal{R}) \rightarrow L^{2}(\mathcal{R}), b=\frac{1}{2}\left(\frac{\partial}{\partial u}+W(u)\right), f: \mathcal{C}^{2} \rightarrow \mathcal{C}^{2}, f=\left(\begin{array}{cc}0 & 0 \\ 1 & 0\end{array}\right)$, where the supersymmetric potential $W(u): \mathcal{R} \rightarrow \mathcal{R}$ defined on the given $(i)$ internal world is assumed to be piecewise continuously differentiable function. The commutation and anticommutation relations for these operators 
read $\left[b, b^{+}\right]=W^{\prime}(u), \quad\left\{f, f^{+}\right\}=1$. Employing standard technique, next we define the nilpotent supercharge operators $Q_{u}=Q_{0 u} b \otimes f^{+}=Q_{0 u}\left(\begin{array}{ll}0 & b \\ 0 & 0\end{array}\right), Q_{u}{ }^{+}=Q_{0 u} b^{+} \otimes f=Q_{0 u}\left(\begin{array}{ll}0 & 0 \\ b^{+} & 0\end{array}\right)$, which obey the anticommutation relations $\left\{Q_{u}, Q_{u}\right\}=\left\{Q_{u}{ }^{+}, Q_{u}{ }^{+}\right\}=0$, and act as follows: $Q_{u} \mid n_{B}, n_{F}>$ $\propto\left|n_{B}-1, n_{F}+1>, Q_{u}^{+}\right| n_{B}, n_{F}>\propto \mid n_{B}+1, n_{F}-1>$.

\subsection{The Primary Field}

As alluded to above, we have chosen a simple setting and considered the 'primordial' structures, which are designed to posses certain physical properties satisfying the stated in (Ter-Kazarian, 1884, 1996, 1999a) general rules and have involved in the linkage establishing processes. The processes of their creation and annihilation in the lowest state (the 'regular structures') just are described by the OM formalism. In all the higher states the 'primordial structures' are distorted ones, namely they have undergone the distortion transformations. These transformations yield the 'quark' and "antiquark' fields defined on the simplified geometry (one $u$-channel), and skeletonized for illustrative purposes. Due to geometry realization conditions held in the stable systems of 'primordial structures' they emerge in confined phase. This scheme still should be considered as the preliminary one, which is further elaborated in this subsection to get the physically more realistic picture. The distortion transformation functions are the operators acting in the space of the internal degrees of freedom (colours) and imply the incompatibility relations (Ter-Kazarian, 1884, 1996, 1999a), which hold for both the local and the global distortion rotations. They underly the most important symmetries such as the internal symmetries $U(1), S U(2), S U(3)$, the $S U(2) \otimes U(1)$ symmetry of electroweak interactions, etc.

On these premises, in the enlarged framework of the OMM we define and clarify the fundamental conceptual basis of subquarks (instead of quarks) and their characteristics stemming from the various symmetries of the internal worlds. By this we have arrived at an entirely satisfactory answer to the question of the physical origin of the geometry and fields, the internal symmetries and interactions, as well the principles of relativity, quantum, gauge and subcolour confinement. The OMM formalism has the following features: All the fields including the leptons and quarks, along with the spacetime components have also the MW internal components made of the various constituent subquarks defined on the given internal worlds, such that the internal components are consisted of 'distorted ordinary struc-

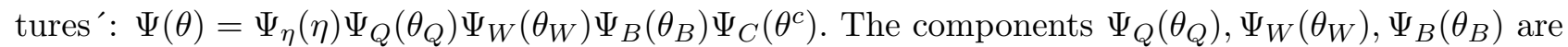
primary massless bare Fermi fields. We assume that this field has arisen from primary field in the lowest state $\left(s_{0}\right)$ with the same field components consisted of 'regular ordinary structures', subject to certain rules (Ter-Kazarian, 1884, 1996, 1999a). Therefore, the primary field defined on $G_{N}$ $\Psi(0)=\Psi_{\eta}(\eta) \Psi_{Q}(0) \Psi_{W}(0) \Psi_{B}(0) \Psi_{C}(0)$ serves as the ready made frame into which the distorted ordinary structures of the same species should be involved. We apply the Lagrangian of this field possessed local gauge invariance written in the notations $\widetilde{L}_{0}(D)=\frac{i}{2}\left\{\bar{\Psi}_{e}(\zeta)^{i} \gamma D_{i} \Psi_{e}(\zeta)-D_{i} \bar{\Psi}_{e}(\zeta)^{i} \gamma \Psi_{e}(\zeta)\right\}$, with the vector indices contracted to form scalars, where $D_{i}=\partial_{i}-i g \mathbf{B}_{\mathbf{i}}(\zeta), \mathbf{B}_{\mathbf{i}}$ are gauge fields. Since the components $\Psi_{B}$ and $\Psi_{C}$ will be of no consequence for a discussion, then we temporarily leave them implicit, namely $i=\eta, Q, W$. The equation of primary field of the MW- structure with nonlinear fermion interactions of the components may be derived from an invariant action in terms of local gauge invariant Lagrangian, which looks like Heisenberg theory: $\widetilde{L}(D)=\widetilde{L}_{0}(D)+\widetilde{L}_{I}+\widetilde{L}_{B}$, provided by the Lagrangians of nonlinear fermion interactions of the components $\widetilde{L}_{I}=\sqrt{2} \widetilde{O}_{1} \otimes L_{I}$, and gauge field $\widetilde{L}_{B}=\sqrt{2} \widetilde{O}_{1} \otimes L_{B}$. The binding interactions are in the form $L_{I}=L_{Q I}+L_{W I}, L_{Q I}=$ $\frac{\lambda}{4}\left(J_{Q L} J_{Q R}^{+}+J_{Q R} J_{Q L}^{+}\right), L_{W I}=\frac{\lambda}{2} S_{W} S_{W}^{+}, L_{B}=-\frac{1}{2} \operatorname{Tr}(\mathbf{B} \overline{\mathbf{B}})=-\frac{1}{2} \operatorname{Tr}\left(\mathbf{B}_{\mathbf{i}} \mathbf{B}_{\mathbf{i}}\right)$, where $J_{Q L, R}=V_{Q} \mp A_{Q}$, $V_{Q(\lambda \alpha)}=\bar{\Psi}_{Q} \gamma_{(\lambda \alpha)} \Psi_{Q}, V_{Q(\lambda \alpha)}^{+}=V_{Q}^{(\lambda \alpha)}=\bar{\Psi}_{Q} \gamma^{(\lambda \alpha)} \Psi_{Q} A_{Q(\lambda \alpha)}=\bar{\Psi}_{Q} \gamma_{(\lambda \alpha)} \gamma_{5} \Psi_{Q}, A_{Q(\lambda \alpha)}^{+}=A_{Q}^{(\lambda \alpha)}=$ $\bar{\Psi}_{Q} \gamma^{5} \gamma^{(\lambda \alpha)} \Psi_{Q}, S_{W}=\bar{\Psi}_{W} \Psi_{W}, \gamma_{\mu}$ and $\gamma_{5}=i \gamma_{0} \gamma_{1} \gamma_{2} \gamma_{3}$ are Dirac matrices. According to Fiertz theorem the interaction Lagrangian $L_{Q I}=\frac{\lambda}{2}\left(V V^{+}-A A^{+}\right)$may be written $L_{Q I}=-\lambda\left(S_{Q} S_{Q}^{+}-P_{Q} P_{Q}^{+}\right)$, provided by $S_{Q}=\bar{\Psi}_{Q} \Psi_{Q}, \quad P_{Q}=\bar{\Psi}_{Q} \gamma_{5} \Psi_{Q}$. Hence $\widetilde{L}(D)=\sqrt{2} \widetilde{O}_{1} \otimes L(D), L(D)=L_{\eta}\left(D_{\eta}\right)-L_{Q}\left(D_{Q}\right)-L_{W}\left(D_{W}\right)$, where $L_{\eta}\left(D_{\eta}\right)=L_{\eta}{ }_{0}^{\prime(0)}\left(D_{\eta}\right)-\frac{1}{2} \operatorname{Tr}\left(\mathbf{B}_{\eta} \overline{\mathbf{B}_{\eta}}\right), L_{Q}\left(D_{Q}\right)=L_{Q}{ }_{0}^{\prime(0)}\left(D_{Q}\right)-L_{Q I}-\frac{1}{2} \operatorname{Tr}\left(\mathbf{B}_{\mathbf{Q}} \mathbf{B}_{\mathbf{Q}}\right), L_{W}\left(D_{W}\right)=$ $L_{W}{ }_{0}^{\prime(0)}\left(D_{W}\right)-L_{W I}-\frac{1}{2} \operatorname{Tr}\left(\mathbf{B}_{\mathbf{W}} \mathbf{B}_{\mathbf{W}}^{-}\right)$. Here $L_{\eta}{ }_{0}^{\prime(0)}=\frac{i}{2}\left\{\bar{\Psi} \gamma \widehat{D}_{\eta} \Psi-\bar{\Psi} \gamma \overleftarrow{D}_{\eta} \Psi\right\}=\Psi_{u}{ }^{+} L_{\eta 0}{ }^{(0)} \Psi_{u}$, 
$L_{u}{ }_{0}^{(0)}=\frac{i}{2}\left\{\bar{\Psi} \gamma \widehat{D}_{u} \Psi-\bar{\Psi} \gamma \overleftarrow{D}_{u} \Psi\right\}=\Psi_{\eta}{ }^{+} L_{u 0}^{(0)} \Psi_{\eta}$, and $L_{\eta 0}^{(0)}=\frac{i}{2}\left\{\bar{\Psi}_{\eta} \gamma \widehat{D}_{\eta} \Psi_{\eta}-\bar{\Psi}_{\eta} \gamma \overleftarrow{D}_{\eta} \Psi_{\eta}\right\}$ $L_{u 0}{ }^{(0)}=\frac{i}{2}\left\{\bar{\Psi}_{u} \gamma \widehat{D}_{u} \Psi_{u}-\bar{\Psi}_{u} \gamma \overleftarrow{D}_{u} \Psi_{u}\right\}$. The total Lagrangian has the global $\gamma_{5}$ and local gauge symmetries. We consider only $\gamma_{5}$ symmetry in Q-world, namely $\mathbf{B}_{\mathbf{Q}} \equiv 0$. According to the OMM formalism, it is important to fix the mass shell of the stable MW- structure. It means that we must take at first the variation of the Lagrangian with respect to primary field, then have switched on nonlinear fermion interactions of the components. In other words we take the variation of the Lagrangian with respect to the components on the fixed mass shell.

Reflecting upon the discussed in two previous subsections subject, we conclude that in the framework of MW-geometry, all the fields have composite nontrivial internal structure. The MW structure of primary field is described by the gauge invariant Lagrangian involving nonlinear fermion interactions of the internal field components somewhat similar to the theory by Heisenberg and his co-workers, but still it will be defined on the MW-geometry. This Lagrangian is the whole story since all the complexity of the leptons, quarks and their interactions arises from it. The number of free parameters in this Lagrangian is reduced to primary coupling constant of the nonlinear interaction and gauge coupling. All the fields along with the spacetime component have nontrivial composite internal MW structure. The possible elementary particles are thought to be composite dynamical systems in analogy to quantum mechanical stationary states of compound atom, but, now a dynamical treatment built up on the MW-geometry is quite different and more amenable to qualitative understanding (Ter-Kazarian, 1884, 1996, 1999a). The microscopic structure of leptons, quarks and other particles will be governed by the only possible conjunctions of constituent subquarks implying concrete symmetries.

The hypothesis of existence of the MW structures manifests its virtue by solving some key problems of particle phenomenology, when we attempt to suggest a microscopic approach to the properties of particles and interactions. We consider further the microscopic theory of the unified electroweak interactions (Ter-Kazarian, 1884, 1996, 1999a). It follows that contemporary phenomenological SM is an approximation to the suggested microscopic approach. The condition of realization of the MW connections is arisen due to the symmetry of Q-world of electric charge and embodied in the GellMann-Nishijima relation. During the realization of the MW-structure the symmetries of corresponding internal worlds are unified into more higher symmetry including also the operators of isospin and hypercharge. Such approach enables to conclude that only possible at low-energy the three lepton generations consist of six lepton fields with integer electric and leptonic charges and being free of confinement. Also the three quark generations exist composed of six possible quark fields. They carry fractional electric and baryonic charges and obey confinement condition. The global group unifying all global symmetries of the internal worlds of quarks is the flavour group $S U_{f}(6)$. The Lagrangian of primary field contains only two free parameters, which are the coupling constants of nonlinear fermion and gauge interactions.

Hence, the OMM formalism provides a natural unification of the geometry-yielding the 1) Special and 2) General relativity principles, and the fermion fields serving as the basis for the constituent subquarks.

It has cleared up the physical conditions in which the geometry and particles come into being.

The subquarks emerge in the geometry only in certain permissible combinations utilizing the idea of the 3) Subcolour (subquark) confinement principle, and have undergone the transformations yielding the Internal symmetries and 4) Gauge principle.

Although within considered schemes the subquarks are defined on the internal worlds, however the resulting spacetime components of particles, which we are going to deal with to describe the leptons and quarks defined on the spacetime continuum, are affected by them in such a way that they carry exactly all the quantum numbers of the various constituent subquarks of the given composition. We discussed a class of models of internal symmetries, which reproduce the known phenomenology of electromagnetic, weak and strong interactions (Ter-Kazarian, 1884, 1996, 1999a). In order to save writing we guess it worthwhile to leave the other concepts such as the flavors and so forth with associated fundamental aspects of particle physics for an other treatment. It will not concern us here and must be further discussed. Surely this is an important subject for separate research. 


\section{Microscopic theory of the Standard Model of elementary parti- cles}

We attempt to develop, further, the microscopic theory of the Standard Model of elementary particles (Ter-Kazarian, 1999b, 2001b), which enables an insight to the key problems of particle phenomenology.

\subsection{Objectives of MTSM}

The most important open questions of the SM are as follows: We have no understanding why the SM is as it is? Why is the gauge symmetry? Why is this the particle spectrum? Why the electroweak symmetry breaking sector consists of just one $S U(2)_{L}$ doublet of Higgs bosons as it is in SM? The untested aspects of SM are the mass spectrum of the particles, the mixing patterns and the $\mathrm{CP}$ violation. The latter is introduced through complex Yukawa couplings of fermions to Higgs bosons, resulting in complex parameters in the CKM matrix. The SM contains a large number of arbitrary parameters, while a consistent complete theory would not have so many free parameters.

To address to some of these nagging questions of the SM, the MTSM is developed, wherein the proliferation of lepton and quark flavours prompts us to consider the fields as composites. Certainly, it may seem foolhardy to set up such a picture in the spacetime continuum. The difficulties arisen here are well-known. The first problem is closely related to the expected mass differences of particles, which in this case would be too large $(\geq 1 \mathrm{TeV})$. Another problem concerns the transformations of particles. Our idea is to remove these difficulties by employing MTSM, which is based on the MW-geometry. This theory attempts to answer to some of the above mentioned questions of particle phenomenology. The MTSM enables an insight to the key concepts of particle physics, and to conclude that the leptons are particles with integer electric and leptonic charges and free of confinement, while the quarks carry fractional electric and baryonic charges and imply the confinement. The theoretical significance of the MTSM resides in the microscopic interpretation of all physical parameters. We derive the Gell-MannNishijima relation and the flavour group. The testable implications of the MTSM are given. Finally, we derive a physically more realistic mass spectrum of the leptons and quarks instead of the former one inferred within the simplified scheme.

A theoretical significance of the MTSM, first of all, resides in the microscopic interpretation of all physical parameters. Due to specific structure of the $\mathrm{W}$-world of weak interactions implying the condition of realization of the MW-connections, the spanning takes place, which underlies the $\mathrm{P}$-violation in $\mathrm{W}$-world. It is expressed in the reduction of initial symmetry of the right-handed subquarks. Such reduction is characterized by the Weinberg mixing angle with the value fixed at $30^{0}$. It gives rise to the expanded local symmetry $S U(2) \otimes U(1)$, under which the left-handed fermions transform as six independent $S U(2)$ doublets, while the right-handed fermions transform as twelve independent singlets. Due to vacuum rearrangement in Q-world the Yukawa couplings arise between the fermion fields and corresponding isospinor-scalar $\varphi$-meson in conventional form.

\subsection{Higgs bosons and Electroweak symmetry breaking}

We suggest the microscopic approach to Higgs bosons with self-interaction and Yukawa couplings. It involves the Higgs bosons as the collective excitations of bound quasi-particle iso-pairs. To obtain some feeling about this statement, below we give more detailed explanation.

Tracing a resemblance with the Cooper pairs, within the framework of local gauge invariance of the theory incorporated with the phenomenon of P-violation in weak interactions we suggest a mechanism providing the Bose-condensation of relativistic fermion pairs, which is due to effective attraction between the relativistic fermions caused by the exchange of the mediating induced gauge quanta in the $\mathrm{W}$-world. The rationale for this approach is readily forthcoming from the consideration of gauge transformations of the fields under the P-violation in the $\mathrm{W}$-world $\Psi_{L}^{\prime}(x)=U_{L}(x) \Psi_{L}(x), \quad \Psi_{R}^{\prime}(x)=$ $U_{R}(x) \Psi_{R}(x)$, where the Fourier expansions carried out over corresponding gauge quanta with wave

fourvectors $q_{L}$ and $q_{R} U_{L}(x)=\int \frac{d^{4} q_{L}}{(2 \pi)^{4}} e^{i q_{L} x} U_{L}\left(q_{L}\right), \quad U_{R}(x)=\int \frac{d^{4} q_{R}}{(2 \pi)^{4}} e^{i q_{R} x} U_{L}\left(q_{R}\right)$, and $U_{L}(x) \neq$ $U_{R}(x)$. They induce the gauge transformations implemented upon the $\varphi$-field $\varphi^{\prime}(x)=U(x) \varphi(x)$. 
The matrix of induced gauge transformations may be written down in terms of induced gauge quanta $U(x) \equiv U_{L}^{+}(x) U_{R}(x)=\int \frac{d^{4} q}{(2 \pi)^{4}} e^{i q x} U(q)$, where $q=-q_{L}+q_{R}, \quad q\left(q^{0}, \vec{q}\right)$. In momentum space one gets $\varphi^{\prime}\left(k^{\prime}\right)=\int \frac{d^{4} q}{(2 \pi)^{4}} U(q) \varphi\left(k^{\prime}-q\right)=\int \frac{d^{4} k}{(2 \pi)^{4}} U\left(k^{\prime}-k\right) \varphi(k)$. Conservation of the fourmomentum requires that $k^{\prime}=k+q$. Accordingly, we have $-p_{L}^{\prime}+p_{R}^{\prime}=-p_{L}+p_{R}+q=-p_{L}^{\prime \prime}+p_{R}=-p_{L}+p_{R}^{\prime \prime}$, where $p_{L}^{\prime \prime}=p_{L}-q, \quad p_{R}^{\prime \prime}=p_{R}+q$. Whence the wave vectors of fermions imply the conservation law $\vec{p}_{L}+\vec{p}_{R}=\vec{p}_{L}^{\prime \prime}+\vec{p}_{R}^{\prime \prime}$, characterizing the scattering process of two fermions with effective interaction caused by the mediating induced gauge quanta. We suggest the mechanism for the effective attraction between the fermions in the following manner: Among all induced gauge transformations with miscellaneous gauge quanta we distinguish a special subset with the induced gauge quanta of the frequencies belonged to finite region characterized by the maximum frequency $\frac{\widetilde{q}}{\hbar} \quad\left(\widetilde{q}=\max \left\{q^{0}\right\}\right)$ greater than the frequency of inducing oscillations fermion force $\frac{\bar{E}_{L}-\bar{E}_{L}^{\prime \prime}}{\hbar}<\frac{\widetilde{q}}{\hbar}$. To the extent that this is a general phenomenon, we can expect under this condition the effective attraction (negative interaction) arisen between the fermions caused by exchange of virtual induced gauge quanta if only the forced oscillations of these quanta occur in the same phase with the oscillations of inducing force (the oscillations of fermion density). In view of this we may think of isospinor $\Psi_{L}$ and isoscalar $\Psi_{R}$ fields as the fermion fields composing the iso-pairs with the same conserving net momentum $\vec{p}=\vec{p}_{L}+\vec{p}_{R}$ and opposite spin, for which the maximum number of negative matrix elements of operators composed by corresponding creation and annihilation operators $a_{\vec{p}_{R}^{\prime \prime}}^{+} a_{\vec{p}_{R}} a_{\vec{p}_{L}^{\prime \prime}}^{+} a_{\vec{p}_{L}}$ (designated by the pair wave vector $\vec{p}$ ) may be obtained for coherent ground state with $\vec{p}=\vec{p}_{L}+\vec{p}_{R}=0$. In the mean time the interaction potential reads $V=\sum_{\vec{p}_{R}^{\prime \prime}, \vec{p}_{L}^{\prime \prime}, \vec{p}_{R}, \vec{p}_{L}}\left(a_{\vec{p}_{L}}^{+}\right)^{+} a_{\vec{p}_{R}^{\prime \prime}}^{+}\left(a_{\vec{p}_{L}^{\prime \prime}}^{+}\right) a_{\vec{p}_{R}}=\sum_{\vec{p}_{R}^{\prime \prime}, \vec{p}_{L}^{\prime \prime}, \vec{p}_{R}, \vec{p}_{L}} a_{\vec{p}_{R}^{\prime \prime}}^{+} a_{\vec{p}_{L}^{\prime \prime}}^{+} a_{\vec{p}_{R}} a_{\vec{p}_{L}}$, implying the attraction between the fermions situated in the spherical thin shall near the Fermi surface $V_{\vec{p} \vec{p}^{\prime}}=\left\{\begin{array}{l}-V \text { at }\left|E_{\vec{p}}-E_{F}\right| \leq \widetilde{q}, \quad\left|E_{\vec{p}^{\prime \prime}}-E_{F}\right| \leq \widetilde{q}, \text {. The fermions filled up the Fermi sea block } \\ 0 \text { otherwise }\end{array}\right.$ the levels below Fermi surface. Hence, the fermions are in superconducting state if this condition holds. Otherwise, they are in normal state described by Bloch individual particle model. Hence, the Bose-condensate arises in the $\mathrm{W}$-world as the collective mode of excitations of bound quasi-particle iso-pairs described by the same wave function in the superconducting phase $\Psi=\left\langle\Psi_{L} \Psi_{R}\right\rangle$, where $\langle\cdots\rangle$ is taken to denote the vacuum averaging. The vacuum of the $\mathrm{W}$-world is filled up by such iso-pairs at absolute zero $T=0$. We make a final observation that $\Psi_{R} \Psi_{R}^{+}=n_{R}$ is a scalar density number of right-handed particles. It readily follows that: $\left(\Psi_{L} \Psi_{R}\right)^{+}\left(\Psi_{L} \Psi_{R}\right)=\Psi_{R}^{+} \Psi_{L}^{+} \Psi_{L} \Psi_{R}=$ $\frac{1}{n_{R}} \Psi_{R}^{+} \gamma^{0}\left(\gamma^{0} \Psi_{L}^{+} \Psi_{R}\right)\left(\Psi_{R}^{+} \Psi_{L} \gamma^{0}\right) \gamma^{0} \Psi_{R}=\varphi \varphi^{+}$, where $|\Psi|^{2}=<\varphi \varphi^{+}>=|<\varphi>|^{2} \equiv|\varphi|^{2}$. It is convenient to abbreviate the $\langle\varphi\rangle$ by the symbol $\varphi$. Hence the $\varphi$-meson actually arises as the collective mode of excitations of bound quasi-particle iso-pairs.

\subsection{The Non-Relativistic Approximation}

In the approximation to non-relativistic limit $\left(\beta \ll 1, \Psi_{L} \simeq \Psi_{R}, \gamma^{0} \rightarrow 1\right)$ by making use of Ginzburg-Landau's (GL) phenomenological theory, it is straightforward to write down the free-energy functional for the order parameter in equilibrium superconducting phase in presence of magnetic field. The self-consistent coupled GL-equations are differential equations like Schrödinger and Maxwell equations, which relate the spatial variation of the order parameter $\Psi$ to the vector potential $\vec{A}$ and the current $\vec{j}_{s}$. By means of thermodynamic Green's functions in well defined limit, it is shown that GL-equations are a consequence of the BCS-Bogoliubov microscopic theory of superconductivity. The theoretical significance of these works resides in the microscopic interpretation of all physical parameters of GL-theory. Subsequently these ideas were extended to lower temperatures using a requirement that the order parameter and vector potential vary slowly over distances of the order of the coherence length and that the electrodynamics be local (London limit). Namely, the validity of derived GLG-equations is restricted to the temperature $T$, such $T_{c}-T \ll T_{c}$ and to the local electrodynamics region $q \xi_{0} \ll 1$, where $T_{c}$ is transition temperature, $\xi_{0}$ is coherent length characterizing the spatial extent of the electron pair correlations, $q$ are the wave numbers of magnetic field $\vec{A}$. The most important order parameter $\Psi$, the mass $m_{\Psi}$ and the coupling constant $\lambda_{\Psi}$ figured in GLG-equations read $\Psi(\vec{r})=\frac{(7 \zeta(3) N)^{1 / 2}}{4 \pi k_{B} T_{c}} \Delta(\vec{r}), \Delta(T)=\simeq 3.1 k_{B} T_{c}\left(1-\frac{T}{T_{c}}\right)^{1 / 2}, \xi_{0}=\simeq 0.18 \frac{\hbar v_{F}}{k_{B} T_{c}}, m_{\Psi}^{2}=1.83 \frac{\hbar^{2}}{m} \frac{1}{\xi_{0}^{2}}\left(1-\frac{T}{T_{c}}\right)$, 
A new look at some aspects of geometry, particle physics, inertia, radiation and cosmology

$\lambda_{\Psi}^{2}=1.4 \frac{1}{N(0)}\left(\frac{\hbar^{2}}{2 m \xi_{0}^{2}}\right)^{2} \frac{1}{\left(k_{B} T_{c}\right)^{2}}$. Reviewing the notation $\Delta(\vec{r})$ is the energy gap, $e^{*}=2 e$ is the effective charge, $N(0)$ is the state density at Fermi surface, $N$ is the number of particles per unit volume in normal mode, $v_{F}$ is the Fermi velocity, $m \equiv \Sigma_{Q}=f_{Q}$ is the mass of fermion field. The transition temperature relates to gap at absolute zero $\Delta_{0}$. The estimate for the pair size at $v_{F} \sim 10^{8} \mathrm{~cm} / \mathrm{s}, T_{c} \sim 1$ gives $\xi_{0} \simeq 10^{-4} \mathrm{~cm}$.

\subsection{The Relativistic Treatment}

We start with the Lagrangian of self-interacting fermion field in W-world, which is arisen from the Lagrangian of primary fundamental field after the rearrangement of the vacuum of the Q-world $L_{W}(x)=\frac{i}{2}\left\{\bar{\Psi}_{W}(x) \gamma^{\mu} \partial_{W \mu} \Psi_{W}(x)-\bar{\Psi}_{W}(x) \gamma^{\mu} \overleftarrow{\partial}_{W \mu} \Psi_{W}(x)\right\}-m \bar{\Psi}_{W}(x) \Psi_{W}(x)-$ $\frac{\lambda}{2} \bar{\Psi}_{W}(x)\left(\bar{\Psi}_{W}(x) \Psi_{W}(x)\right) \Psi_{W}(x)$. Here, $m=\Sigma_{Q}$ is the self-energy operator of the fermion field component in Q-world, the suffix $(W)$ just was put forth in illustration of a point at issue. For the sake of simplicity, we also admit $\mathbf{B}_{\mathbf{W}}(x)=0$, but, of course, one is free to restore the gauge field $\mathbf{B}_{\mathbf{W}}(x)$ whenever it will be needed. In lowest order the relation $m \equiv m_{Q} \ll \lambda^{-1 / 2}$ holds. The Lagrangian $L_{W}(x)$ leads to the field equations $(\gamma p-m) \Psi(x)-\lambda(\bar{\Psi}(x) \Psi(x)) \Psi(x)=0, \bar{\Psi}(x)(\gamma \overleftarrow{p}+m)+\lambda \bar{\Psi}(x)(\bar{\Psi}(x) \Psi(x))=0$ where the indices have been suppressed as usual. At non-relativistic limit the function $\Psi$ reads $\Psi \rightarrow e^{i m c^{2} t} \Psi$. In the following, we make use of conventional technique and evaluate the equations. The spirit of the calculation will be to treat interaction between the particles as being absent everywhere except the thin spherical shell $2 \widetilde{q}$ near the Fermi surface. The Bose condensate of bound particle iso-pairs occurred at zero momentum. The scattering processes between the particles are absent. This method allows oneself to extend the study up to limit of temperatures, such that $T_{c}-T \ll T_{c}$, by making use of thermodynamic Green's function.

\subsection{Lagrangian of Electroweak Interactions; The Transmission of the Electroweak Symmetry Breaking From the $W$-World to Spacetime Continuum}

The results obtained within the previous subsections enable us to trace unambiguously rather general scheme of unified electroweak interactions, where the self-interacting isospinor scalar Higgs bosons have arisen as the collective modes of excitations of bound quasi-particle iso-pairs on the internal $W$-world. But, at the very first we remind some features allowing us to write down the final Lagrangian of electroweak interactions.

1. During the realization of the MW connections of weak interacting fermions under the action of the $Q$-world the P-violation compulsory occurred in the $\mathrm{W}$-world incorporated with the symmetry reduction characterized by the Weinberg mixing angle with the fixed value at $30^{0}$. This gives rise to the local symmetry $S U(2) \otimes U(1)$, under which the left-handed fermions transformed as six independent doublets, while the right-handed fermions transformed as twelve independent singlets.

2. Due to vacuum rearrangement in Q-world the Yukawa couplings arise between the fermion fields and corresponding isospinor-scalar $\varphi$ - meson in conventional form.

3. In the framework of suggested mechanism providing the effective attraction between the relativistic fermions caused by the exchange of the mediating induced gauge quanta in W-world, the self-interacting isospinor-scalar Higgs bosons arise as Bose-condensate, namely the $S U(2)$ multiplets of spinless $\varphi$-meson fields coupled to the gauge fields in a gauge invariant way. Thus, in the Lagrangian of $\varphi$-meson with the degenerate vacuum of the $\mathrm{W}$-world the symmetry-breaking Higgs boson is counted off from the gap symmetry restoring value as the point of origin.

In view of this the total Lagrangian ensues, and it is now invariant under the local symmetry $S U(2) \otimes U(1)$, where a set of gauge fields are coupled to various multiplets of fields among which is also a multiplet of Higgs boson. Subsequently, we separate a piece of Lagrangian containing only the fields defined on four dimensional Minkowski flat spacetime continuum $M_{4}$. To facilitate writing we shall forbear here to write out the piece of Lagrangian containing the terms of other fermion generations than one, as it is a somewhat lengthy and so standard. But, in the mean time, we shall retain the explicit terms of Higgs bosons arisen on the internal $W$-world to emphasize the specific mechanism of the electroweak symmetry breakdown discussed below. The resulting Lagrangian reads $L=-\frac{1}{2} \operatorname{Tr} G_{\mu \nu} G^{\mu \nu}-\frac{1}{4} F_{\mu \nu} F^{\mu \nu}+i \bar{L} \hat{D} L+i \bar{e}_{R} \hat{D} e_{R}+i \bar{\nu}_{R} \hat{D} \nu_{R}+\left|D_{W \mu} \varphi\right|^{2}-\frac{1}{2} \lambda_{\varphi}^{2}\left(|\varphi|^{2}-\frac{1}{2} \eta_{\varphi}^{2}\right)^{2}-$ 
A new look at some aspects of geometry, particle physics, inertia, radiation and cosmology

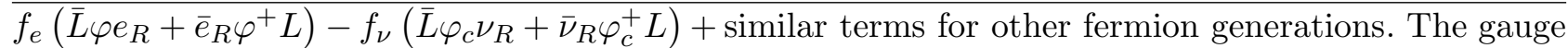
fields $\mathbf{A}_{\mu}(x)$ and $B_{\mu}(x)$ associate respectively with the groups $S U(2)$ and $U(1)$, where the gauge covariant curls are $F_{\mu \nu}, G_{\mu \nu}$. The corresponding gauge covariant derivatives are in standard form. One took into account corresponding values of the operators $\mathbf{T}$ and $Y$ for left- and right-handed fields, and for isospinor $\varphi$-meson. The Yukawa coupling constants $f_{e}$ and $f_{\nu}$ are inserted in subsec.6.10. Since the electroweak symmetry is at any rate only approximate, the test of the theory will depend on its ability to account for its breaking as well. here the MSM creates a particular incentive for the study of such a breaking. Then, just it remains to see how can such Higgs bosons arisen on the internal $W$-world break the gauge symmetry down in $M_{4}$ and lead to masses of the spacetimecomponents of the MW-fields? It is remarkable to see that the suggested MSM, in contrast to the SM, predicts the transmission of electroweak symmetry breaking from the $W$-world to the $M_{4}$ spacetime continuum. Actually, in standard scenario for the simplest Higgs sector, a gauge invariance of the Lagrangian is broken when the $\varphi$-meson fields acquire a VEV $\eta_{\varphi} \neq 0$ in the $W$-world. While the mass $m_{\varphi}$ and coupling constant $\lambda_{\varphi}$ are in the standard form. The spontaneous breakdown of symmetry is vanished at $\eta_{\varphi}^{2}\left(\lambda, T>T_{c \mu}\right)<0$. When this doublet obtains a VEV, three of the gauge fields $Z_{W \mu}^{0}, W_{W \mu}^{ \pm}$acquire masses. These fields are the $W$-components of the mesons mediating the weak interactions. This mechanism does not disturb the renormalizability of the theory . In approximation to lowest order $f=\Sigma_{Q} \simeq m_{Q} \ll \lambda^{-1 / 2}\left(\lambda^{-1}=\frac{m p_{0}}{2 \pi^{2}} \ln \frac{2 \widetilde{\omega}}{\Delta_{0}}\right)$, the Lagrangian produce the Lagrangian of phenomenological SM, where at $f \sim 10^{-6}$ one gets $\lambda \ll 10^{12}$. In standard scenario the lowest pole $m_{Q}$ of the self-energy operator $\Sigma_{Q}$ has fixed the whole mass spectrum of the SM particles. But, in general, one must also take into account the mass spectrum of expected various collective excitations of bound quasi-particle pairs produced by higher-order interactions as a 'superconductive' solution obtained from a nonlinear spinor field Lagrangian of the $Q$-component possessed $\gamma_{5}$ invariance. These states must be considered as a direct effect of the same primary nonlinear fermion interaction which provides the mass of the $Q$-component of Fermi field, which itself is a collective effect. They would manifest themselves as stable or unstable states. The general features of mass spectrum of the collective excitations and their coupling with the fermions are discussed through the use of the BetheSalpeter equation handled in the simplest ladder approximation incorporated with the self-consistency conditions, when one is still left with unresolved divergence problem. One can reasonably expect that these results for the bosons of small masses at low energy compared to the unbound fermion states are essentially correct in spite of the very simple approximations. Therein, some bound states are predicted too the obtained mass values of which are rather high, and these states should decay very quickly. The high-energy poles may in turn determine the low-energy resonances. All this prompt us to expect that the other poles different from those of lowest one in turn will produce the new heavy SM family partners. Hence one would expect a second important phenomenological implication of the MSM.

\subsection{Quark flavour mixing and the Cabibbo angles}

An implication of quark generations into general scheme will be carried out in the same way of the leptons. But before proceeding further that it is profitable to enlarge it by the additional assumption without asking the reason behind it:

The MW components imply ${ }^{i} \bar{\psi}_{u}{ }^{A}\left(\cdots, \theta_{i_{1}}, \cdots \theta_{i_{n}}, \cdots\right){ }^{j} \psi_{u}{ }^{B}\left(\cdots, \theta_{i_{1}}, \cdots \theta_{i_{n}}, \cdots\right)=$

$\delta_{i j} \sum_{l=i_{1}, \ldots, i_{n}} f_{i l}^{A B}{ }^{i}\left(\bar{q}_{l} q_{l}\right)$, namely, the contribution of each individual subquark ${ }^{i} q_{l}$, into the component of given world $(i)$ is determined by the partial formfactor $f_{i l}^{A B}$. Under the group $S U(2) \otimes U(1)$ the lefthanded quarks transform as three doublets, while the right-handed quarks transform as independent singlets except of following differences:

1. The values of weak-hypercharge of quarks are changed due to their fractional electric charges $q_{L}: Y^{w}=\frac{1}{3}, \quad u_{R}: Y^{w}=\frac{4}{3}, \quad d_{R}: Y^{w}=-\frac{2}{3}$ etc.

2. All Yukawa coupling constants have nonzero values.

3. An appearance of quark mixing and Cabibbo angle, which is unknown in the scope of standard model.

4. An existence of CP-violating phase in unitary matrix of quark mixing. We shall discuss it in the 
next section.

In previous section we attempt to give an explanation to quark mixing and Cabibbo angle. We consider this problem, for simplicity, on the example of four quarks $u, d, s, c$. The further implication of all quarks would complicate the problem only in algebraic sense. Instead of mixing of the $d^{\prime}$ and $s^{\prime}$ it is convenient to consider a quite equivalent mixing of $u^{\prime}$ and $c^{\prime}$. Similar formulas can be worked out for the other mixings. Hence, the nonzero value of Cabibbo angle arises due to nonzero coupling constant $f_{u^{\prime} c^{\prime}}$. The problem is to calculate all coupling constants $f_{u^{\prime} c^{\prime}}, f_{c^{\prime} t^{\prime}}$, and $f_{t^{\prime} u^{\prime}}$ generating three Cabibbo angles $\tan 2 \theta_{3}=\frac{2 f_{u^{\prime} c^{\prime}}}{f_{c^{\prime}}-f_{u^{\prime}}}, \tan 2 \theta_{1}=\frac{2 f_{c^{\prime} t^{\prime}}}{f_{t^{\prime}}-f_{c^{\prime}}}, \tan 2 \theta_{2}=\frac{2 f_{t^{\prime} u^{\prime}}}{f_{u^{\prime}}-f_{t^{\prime}}}$. Since the Q-components of the quark fields $u^{\prime}, c^{\prime}$ and $t^{\prime}$ contain at least one identical subquark, the partial formfactors $\bar{f}_{i}$, as well then all coupling constants, acquire nonzero values causing a quark mixing with the Cabibbo angles. Therefore, the unimodular orthogonal group of global rotations arises, and the quarks $u^{\prime}, c^{\prime}$ and $t^{\prime}$ come up in doublets $\left(u^{\prime}, c^{\prime}\right),\left(c^{\prime}, t^{\prime}\right)$, and $\left(t^{\prime}, u^{\prime}\right)$. For the leptons these formfactors equal zero $\bar{f}_{i}^{l e p t} \equiv 0$, namely the lepton mixing is absent. In conventional notation $\left(\begin{array}{c}u^{\prime} \\ d\end{array}\right)_{L},\left(\begin{array}{c}c^{\prime} \\ s\end{array}\right)_{L},\left(\begin{array}{c}t^{\prime} \\ b\end{array}\right)_{L} \rightarrow$ $\left(\begin{array}{c}u \\ d^{\prime}\end{array}\right)_{L},\left(\begin{array}{c}c \\ s^{\prime}\end{array}\right)_{L},\left(\begin{array}{c}t \\ b^{\prime}\end{array}\right)_{L}$, which gives rise to $f_{u^{\prime} c^{\prime}} \rightarrow f_{d^{\prime} s^{\prime}}, f_{c^{\prime} t^{\prime}} \rightarrow f_{s^{\prime} b^{\prime}}, f_{t^{\prime} u^{\prime}} \rightarrow f_{b^{\prime} d^{\prime}}, f_{u^{\prime}} \rightarrow f_{d^{\prime}}$, $f_{c^{\prime}} \rightarrow f_{s^{\prime}}, f_{t^{\prime}} \rightarrow f_{b^{\prime}}, f_{d} \rightarrow f_{u}, f_{s} \rightarrow f_{c}, f_{b} \rightarrow f_{t}$.

\subsection{The CP-violating phase}

The required magnitude of the $\mathrm{CP}$-violating complex parameter $\varepsilon$ depends upon the specific choice of theoretical model for explaining the $K_{2}^{0} \rightarrow 2 \pi$ decay. From the experimental data it is somewhere $|\varepsilon| \simeq 2.3 \times 10^{-3}$. In the framework of Kobayashi-Maskawa (KM) parametrization of unitary matrix of quark mixing, this parameter may be expressed in terms of three Eulerian angles of global rotations in the three dimensional quark space and one phase parameter. We attempt to derive the KMmatrix with an explanation given to an appearance of the CP-violating phase. Recall that during the realization of $\mathrm{MW}$ - structure the $\mathrm{P}$-violation compulsory occurred in the $\mathrm{W}$-world provided by the spanning. The three dimensional effective space $W_{v}^{\text {loc }}(3)$ arises as follows:

$$
\begin{aligned}
& W_{v}^{l o c}(3) \ni q_{v}^{(3)}=\left(\begin{array}{c}
q_{R}^{w}(\vec{T}=0) \\
q_{L}^{w}\left(\vec{T}=\frac{1}{2}\right)
\end{array}\right) \equiv
\end{aligned}
$$

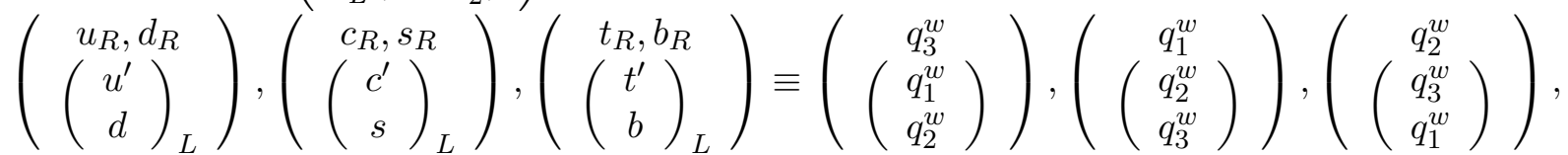

where the subscript $(v)$ formally specifies a vertical direction of multiplet, the subquarks $q_{\alpha}^{w}(\alpha=1,2,3)$ associate with the local rotations around corresponding axes of three dimensional effective space $W_{v}^{l o c}(3)$. The local gauge transformations $f_{e x p}^{v}$ are implemented upon the multiplet $q_{v}^{(3)}=f_{\text {exp }}^{v} q_{v}^{(3)}$, where $f_{\text {exp }}^{v} \in S U^{l o c}(2) \otimes U^{l o c}(1)$. If for the moment we leave it intact and make a closer examination of the content of the middle row, then we distinguish the other symmetry arisen along the horizontal line $(h)$. Hence, we may expect a situation similar to those of previous section will be held in present case. The procedure just explained therein can be followed again. We have to realize that due to the specific structure of $\mathrm{W}$-world implying the condition of realization of the MW connections with $\vec{T} \neq 0, \quad Y^{w} \neq 0$, the subquarks $q_{\alpha}^{w}$ tend to be compulsory involved into triplet. They form one "doublet" $\vec{T} \neq 0$ and one singlet $Y^{w} \neq 0$. Then the quarks $u_{L}^{\prime}, c_{L}^{\prime}$ and $t_{L}^{\prime}$ form a $S O^{g l}(2)$ "doublet" and a $U^{g l}(1)$ singlet $\left(\left(u_{L}^{\prime}, c_{L}^{\prime}\right) t_{L}^{\prime}\right) \equiv\left(\left(q_{1}^{w}, q_{2}^{w}\right) q_{3}^{w}\right) \equiv q_{h}^{(3)} \in W_{h}^{g l}(3),\left(u_{L}^{\prime},\left(c_{L}^{\prime}, t_{L}^{\prime}\right)\right) \equiv\left(q_{1}^{w},\left(q_{2}^{w}, q_{3}^{w}\right)\right)$, $\left(\left(t_{L}^{\prime}, u_{L}^{\prime}\right) c_{L}^{\prime}\right) \equiv\left(\left(q_{3}^{w}, q_{1}^{w}\right), q_{2}^{w}\right)$. Here $W_{h}^{g l}(3)$ is the three dimensional effective space in which the global rotations occur. They are implemented upon the triplets through the transformation matrix $f_{\text {exp }}^{h}$ : $q_{h}^{\prime(3)}=f_{\text {exp }}^{h} q_{h}^{(3)}$, which reads $f_{\text {exp }}^{h}=\left(\begin{array}{ccc}f_{33} & 0 & 0 \\ 0 & c & s \\ 0 & -s & c\end{array}\right)$ in the notation $c=\cos \theta, \quad s=\sin \theta$. This implies the incompatibility relation $\left\|f_{\text {exp }}^{h}\right\|=f_{33}\left(f_{11} f_{22}-f_{12} f_{21}\right)=f_{33} \varepsilon_{123} \varepsilon_{123}\left\|f_{\text {exp }}^{h}\right\| f_{33}^{*}$. That is $f_{33} f_{33}^{*}=1$, or $f_{33}=e^{i \delta}$ and $\left\|f_{\text {exp }}^{h}\right\|=1$. The general rotation in $W_{h}^{g l}(3)$ is described by Eulerian three angles $\theta_{1}, \theta_{2}, \theta_{3}$. If we put the arisen phase only in the physical sector then a final KM-matrix of quark 
flavour mixing would result. The CP-violating parameter $\varepsilon$ approximately is written $\varepsilon \sim s_{1} s_{2} s_{3} \sin \delta \neq$ 0 . Thus, while the spanning $W_{v}^{\text {loc }}(2) \rightarrow W_{v}^{\text {loc }}(3)$ underlies the P-violation and the expanded symmetry $G_{v}^{l o c}(3)=S U^{l o c}(2) \otimes U^{l o c}(1)$, the CP-violation stems from the similar spanning $W_{h}^{g l}(2) \rightarrow W_{h}^{g l}(3)$ with the expanded global symmetry group.

\subsection{Result}

The testable solid implications. If the MTSM proves viable it becomes an crucial issue to hold in experiments the testable solid implications given in, which are drastically different from those of conventional models. Actually, the MTSM rejects drastically any expectation of discovery of any Higgs boson as an elementary particle in $M_{4}$, but in the same time it expects to include a rich spectrum of new particles at higher energies. Namely, if the MTSM proves viable it becomes an crucial issue to hold in experiments the following two solid tests:

1) The Higgs bosons never could emerge as an 'elementary' (apart the pole of composite structure) particle at experiments in spacetime continuum $M_{4}$, nor at any energy range, since these bosons have to arise only on the internal $W$-world and, thus, they produced the electroweak symmetry breakdown in the $W$-world with the subsequent transmission of it to $M_{4}$.

2) For each of the three SM families of quarks and leptons there are corresponding heavy family partners with the same quantum numbers and common mass-shift coefficients $(1+k)$ given for the lowenergy poles at $k_{1}>\sqrt{2}, \quad k_{2}=\sqrt{8 / 3}$ and $k_{3}=2$, lying far above the electroweak scale, respectively, at the energy threshold values: $E_{1}>(419.6 \pm 12.0) \mathrm{GeV}, \quad E_{2}=(457.6 \pm 13.2) \mathrm{GeV}$ and $E_{3}=(521.4 \pm$ 15.0) $\mathrm{GeV}$.

To see its nature, now we may estimate the energy threshold of creation of such heavy family partners using the results far obtained (Nambu \& Jona-Lasinio, 1961). It is therefore necessary under the simplifying assumption to consider in the $Q$-world a composite system of dressed fermion $\left(N_{*}\right)$ made of the unbound fermion $(N)$ coupled with the different kind two-fermion bound states $(N \bar{N})$ at low energy, which all together represent the primary manifestation of the fundamental interaction. Such a dressed fermion would have a total mass $m_{*} \simeq m_{Q}+\mu$, where $m_{Q}$ and $\mu$ are the masses, respectively, of the unbound fermion and the bound state. According to the general discussion of the mass spectrum of the collective excitations given in (Nambu \& Jona-Lasinio, 1961), here we are interested only in the following low-energy bound states written explicitly in spectroscopic notation $\left({ }^{1} S_{0}\right)_{N=0}, \quad\left({ }^{1} S_{0}\right)_{N= \pm 2}, \quad\left({ }^{3} P_{1}\right)_{N=0}$ and $\left({ }^{3} P_{0}\right)_{N=0}$ with the expected masses $\mu=0, \quad>\sqrt{2} m_{Q}, \quad \sqrt{\frac{8}{3}} m_{Q}$ and $2 m_{Q}$, respectively, where the subscript $N$ indicates the nucleon number. One notes the peculiar symmetry existing between the pseudoscalar and the scalar states that the first has zero mass and binding energy $2 m_{Q}$, while the opposite holds for the scalar state. When the next pole $m_{*}$ to the lowest one $m_{Q}$ will be switched on, then due to the Yukawa couplings the all fermions will acquire the new masses with their common shift $\frac{m_{*}}{m_{Q}} \equiv 1+k$ held upwards along the energy scale. To fix the energy threshold value all we have to do then is choose the heaviest member among the SM fermions, which is the top quark, and to set up the quite obvious formula $E \geq E_{0} \equiv m_{t^{\prime}} c^{2}=(1+k) m_{t} c^{2}$, where $m_{t}$ is the mass of the top quark. The top quark observed firstly in the two FNAL $p \bar{p}$ collider experiments in 1995, has the mass turned out to be startlingly large $m_{t}=(173.8 \pm 5.0) \mathrm{GeV} / \mathrm{c}^{2}$ compared to all the other SM fermion masses [125]. Thus, we get the most important energy threshold scale estimate where the heavy partners of the SM extra families of quarks and leptons likely would reside at: $E_{1}>(419.6 \pm 12.0) \mathrm{GeV}, \quad E_{2}=(457.6 \pm 13.2) \mathrm{GeV}$ and $E_{3}=(521.4 \pm 15.0) \mathrm{GeV}$, corresponded to the next nontrivial poles are written: $k_{1}>\sqrt{2}, \quad k_{2}=\sqrt{8 / 3}$ and $k_{3}=2$, respectively. We recognize well that the general results obtained in [95], however, model-dependent and may be considerably altered, especially in the high energy range by using better approximation. In present state of the theory it seemed to be a bit premature to get exact high energy results, which will be important subject for the future investigations. But, in the same time we believe that the approximation used in (Nambu \& Jona-Lasinio, 1961) has enough accuracy for the low-energy estimate made above. Anyhow, it is for one thing, the new scale where the family partners reside will be much higher than the electroweak scale and thus these heavy partners lie far above the electroweak scale.

Remarks: As alluded to above, the Higgs boson does its work of breaking of the electroweak 
symmetry in the $W$-world. The physical pole of formation of such a process, produced by compositeness of this boson in $W$-world, has afterwards transmitted to the $M_{4}$. Then, at the well known experiments performed by ATLAS and CMS Collaborations, announcing the discovery of Higgs boson in 2011- 2013 based on the finding of the excess of events over the background prediction around $125 \mathrm{GeV}$, one has just detected only this pole (maybe of composite structure) with the measurements of its subsequent decay to vector bosons in $M_{4}$, but not the Higgs as the elementary particle at all. It is similar to the case when quarks produced the reactions of elementary particles in $M_{4}$, but in the same time they obeyed Color Confinement principle, and that they appeared in spacetime continuum only in singlet combinations.

Regarding to the last phenomenological implication of the MTSM, it is remarkable that the similar in many respects prediction is made in somewhat different context by Adler (Adler, 2021) within a phenomenological scheme of a compositeness of the quarks and leptons. It based on the generic group theoretical framework of rishon type models exploring the preon constituents. But, therein a present, a bit premature, state of the theory does not allow the exact estimate of this scale. Although one admits that such a scale could be much higher than electroweak scale, however, it is also necessary special argumentations in support of validity of this prediction in the case if this scale has turned out to be low enough, namely, if these heavy partners lie not too far above the electroweak scale. Even thus, one must not worry for the existence of 6 heavy flavors, which is then marginally compatible with the current LEP data. Thus, which of these schemes above, if any, is realized either exactly or at least approximately in nature remains to be seen in the years to come.

Our approach still should be considered as a preliminary one, wherein we have contended ourselves with a rather modest task and do not profess to have any clear-cut answers to all the problems of particle physics, the complete picture of which is largely beyond the scope of the present paper. The only argument that prompts us to consider present approach seriously is the remarkable feature that the most important properties of particle phenomenology can be derived naturally within its framework. Therefore we hope that it will be an attractive basis for the future theory. Although many key problems are elucidated within outlined approach, nevertheless some issues still remain to be solved. For the details of theoretical apparatus of MTSM, see (Ter-Kazarian, 1999b, 2001b).

\section{Supersymmetric Extension of MTSM}

\subsection{Objectives}

There is an important line of reasoning which supports the side of supersymmetrization of the SM, i.e., there are two well known principal issues which remain open in the SM. The first is the vacuum zero point energy problem standing before any quantum field theory. Second is often referred to as the problem of quadratic divergences or the hierarchy and naturalness problem (the dimensional analysis problem) arisen as the quadratic growth of the Higgs boson mass beyond tree level in perturbation theory, namely, the extreme difference in energy scales in the theory is inconsistent in the fundamental scalar sector. This is strong indication for the physics beyond SM. These last two problems can be solved by extending the symmetry of the theory to supersymmetry, which is believed in conventional physics to be manifest at energies in the TeV range. Given the SUSY requiring doubling the number of all the particles by their SUSY partners (sparticles), the quantum radiative corrections may cancel because some loop diagrams vanish due to cancellation between bosons and fermions since they have opposite signs. Then, if the SUSY is present in the TeV range, the masses of the Higgs bosons are no more unstable than fermion masses, whose smallness is natural and hold due to the approximate chiral symmetries. In this manner, its simplest form, SUSY solves the technical aspects of the hierarchy problem as well as the zero point energy problem, when due to power of the boson-fermion cancellation the zero point energy of the fermions exactly cancels that of the bosons and the degeneracy is not arisen. Therefore, in usual, the SM should be regarded as an effective low energy field theory valid up to the energy range smaller than a few hundred GeVs.

However, the SUSY in turn introduces its own set of difficulties. Despite the beautiful mathematical features of SUSY theories and that the SUSY has been theoretically invented almost three decades ago, but a physically realistic realization of SUSY had not been achieved yet and this principal problem was 
ever since much the same as now. In all suggested SUSY theories the supercharges have been inserted in ad hoc manner directly into the four-dimensional spacetime continuum adding a new structure, i.e., a new four odd fermionic dimensions. In fact, a physical essence of the basic concept of supercharge remains unknown and, therefore, the physical theory is beset by various difficulties. Perhaps the most discouraging and disturbing feature of the general class of proposed SUSY theories is the absence at the moment of a solid experimental motivation of supersymmetry, i.e., there is not a direct experimental evidence for the existence of any of the numerous new sparticles predicted by such theories. It is clear, then, that SUSY cannot be an exact symmetry in nature but has to be realized at least in broken phase. The last one is the least understood aspect of such theories. The spontaneously broken SUSY should be ruled out at once since it runs into phenomenological difficulties. One of the viable way out from this situation is an explicit breaking of the global SUSY. A generic parametrization of this phenomenon introduces the much larger free parameter space $(\simeq 124)$ in the models of minimal supersymmetric extension of the SM (MSSM-124). Thus, it is important to develop the other schemes that attempt to reduce the number of free parameters. The conventional SUSY theories predict that the sparticles must reside in the $\mathrm{TeV}$ range. All such arguments that nature is supersymmetric, and that SUSY is broken at scales not too different than the weak scale, are theoretical. The next generation of experiments at Fermilab and CERN will explore this energy range, where at least some of sparticles are expected to be found. All this variety prompts us, further, to adopt the idea that perhaps a more deeper level of organization of physical world may be existed. In the light of current status of particle physics, any new more elaborated outlook seems worthy of investigation.

\subsection{The superfield content of MSMSM and the resulting SUSY Lagrangian}

The results obtained in the previous sections enable us to trace unambiguously rather general scheme of MSMSM, which is essentially a straightforward and viable supersymmetrization of the MSM where we want to keep the number of superfields and interactions as small as possible. To build up the MSMSM the major point is to define its superfield content. Below we recall some important features allowing us to write the resulting Lagrangian of MSMSM.

- Within the MSM, during the realization of MW connections of weak interacting fermions the $\mathrm{P}$-violation compulsory occurred in $\mathrm{W}$-world incorporated with the symmetry reduction. It has characterized by the Weinberg mixing angle with the fixed value at $30^{\circ}$. This gives rise to the local symmetry $S U(2) \otimes U(1)$, under which the left-handed fermions transformed as six independent doublets, while the right-handed fermions transformed as twelve independent singlets.

- Due to vacuum rearrangement in Q-world the Yukawa couplings arise between the fermion fields and corresponding isospinor-scalar $H$-mesons in conventional form.

- In the framework of suggested mechanism, providing the effective attraction between the relativistic fermions caused by the exchange of the mediating induced gauge quanta in $\mathrm{W}$-world, the two complex self-interacting isospinor-scalar Higgs doublets $\left(H_{u}, H_{d}\right)$ as well as their spin- $\frac{1}{2}$ SUSY partners $\left(\widetilde{H}_{u}, \widetilde{H}_{d}\right)$ Higgsinos arise as the Bose-condensate. Taking into account this slight difference from the MSM arisen in the field content of MSMSM in the Higgs sector, we must explicitly write in the supersymmetric Lagrangian also the piece containing these fields coupled to the gauge fields in a gauge invariant way, when the symmetry-breaking Higgs bosons are counted off from the gap symmetry-restoring value as the point of origin.

- The gauge group of MSMSM is the same $S U_{c}(3) \otimes S U(2)_{L} \otimes U(1)$ as in the MSM, which requires a colour octet of vector superfields $V^{a}$, a weak triplet $V^{(\delta)}$ and a hypercharge singlet $V$. Thus, the kinetic terms of all the fields now fixed by gauge invariance $L=\int d^{4} \theta \widetilde{\Phi}_{c h}^{+}\left(e^{g_{1} V T+g_{2} V^{(\delta)}} \dot{T}^{(\delta)}+g_{3} V^{a} T^{a}\right) \widetilde{\Phi}_{c h}+$ $\left[\int d^{2} \theta \frac{1}{4}\left(W W+W^{(\delta)} W^{(\delta)}+W^{a} W^{a}\right)+\right.$ h.c.], where $\widetilde{\Phi}_{c h}$ is the matter superfields, $T, T^{(\delta)}, T^{a}$ are the generators of appropriate representations of the gauge group. The superpotential determines the scalar potential $V\left(A, A^{*}\right)=\frac{1}{2} g_{1}^{2} D^{2}+\frac{1}{2} g_{2}^{2} D^{\delta 2}+\frac{1}{2} g_{2}^{2} D^{a 2}+|P|^{2}$, where the functions $D$ and $P$ are given above.

- By the index $I=1,2,3$ in the MW-SUSY Lagrangian will be labeled the three families of chiral quarks $q_{L}^{I}, u_{R}^{I}, d_{R}^{I}$, and chiral leptons $l_{L}^{I}, e_{R}^{I}$, where all of them are Weyl fermions. A SUSY requires the presence of supersymmetric partners which form supermultiplets with known particles, i.e., for every field of SM there is a superpartner with the exact same gauge quantum numbers. Then, the 
A new look at some aspects of geometry, particle physics, inertia, radiation and cosmology

quarks and leptons are promoted to chiral multiplets by adding scalar (spin-0) squarks $\left(\widetilde{q}_{L}^{I}, \widetilde{u}_{R}^{I}, \widetilde{d}_{R}^{I}\right)$ and sleptons $\left(\widetilde{l}_{L}^{I}, \widetilde{e}_{R}^{I}\right)$ to the spectrum. The gauge bosons are promoted to vector supermultiplet by adding their SUSY partners gauginos $\left(\operatorname{spin}-\frac{1}{2}\right)(\widetilde{G}, \widetilde{W} \widetilde{B})$ to the spectrum. A content of superfields of MSMSM presents in Table 1:

\begin{tabular}{|c|c|c|c|c|c|c|c|}
\hline & supermultiplet & $F$ & $B$ & $S U_{c}(3)$ & $S U(2)_{L}$ & $U(1)_{Y}$ & $U(1)_{e m}$ \\
\hline \multirow[t]{3}{*}{ quarks } & $Q_{L}^{I}=\left(\begin{array}{c}U_{L}^{I} \\
D_{L}^{I}\end{array}\right)$ & $q_{L}^{I}$ & $\widetilde{q}_{L}^{I}$ & 3 & 2 & $1 / 6$ & $\left(\begin{array}{l}2 / 3 \\
-1 / 3\end{array}\right)$ \\
\hline & $U_{R}^{I}$ & $u_{R}^{I}$ & $\widetilde{u}_{R}^{I}$ & $\overline{3}$ & 1 & $-2 / 3$ & $-2 / 3$ \\
\hline & $D_{R}^{I}$ & $d_{R}^{I}$ & $\widetilde{d}_{R}^{I}$ & $\overline{3}$ & 1 & $1 / 3$ & $1 / 3$ \\
\hline \multirow[t]{2}{*}{ leptons } & $L_{L}^{I}=\left(\begin{array}{c}\mathcal{N}_{L}^{I} \\
E_{L}^{I}\end{array}\right)$ & $l_{L}^{I}$ & $\widetilde{l}_{L}^{I}$ & 1 & 2 & $-1 / 2$ & $\left(\begin{array}{l}0 \\
-1\end{array}\right)$ \\
\hline & $E_{R}^{I}$ & $e_{R}^{I}$ & $\widetilde{e}_{R}^{I}$ & 1 & 1 & 1 & 1 \\
\hline \multirow[t]{2}{*}{ Higgs } & $H_{d}=\left(\begin{array}{c}H_{d}^{0} \\
H_{d}^{-}\end{array}\right)$ & $\left(\begin{array}{l}\widetilde{h}^{0} \\
\widetilde{h}^{-}\end{array}\right)$ & $\left(\begin{array}{l}h_{d}^{0} \\
h_{d}^{-}\end{array}\right)$ & 1 & 2 & $-1 / 2$ & $\left(\begin{array}{l}0 \\
-1\end{array}\right)$ \\
\hline & $H_{u}=\left(\begin{array}{c}H_{u}^{+} \\
H_{u}^{0}\end{array}\right)$ & $\left(\begin{array}{l}\widetilde{h}^{+} \\
\widetilde{h}^{0}\end{array}\right)$ & $\left(\begin{array}{l}h_{u}^{+} \\
h_{u}^{0}\end{array}\right)$ & 1 & 2 & $1 / 2$ & $\left(\begin{array}{l}1 \\
0\end{array}\right)$ \\
\hline \multirow{3}{*}{$\begin{array}{l}\text { gauge } \\
\text { bosons }\end{array}$} & G & $\widetilde{G}$ & $G$ & 8 & 1 & 0 & 0 \\
\hline & W & $\widetilde{W}$ & $W$ & 1 & 3 & 0 & $(0, \pm 1)$ \\
\hline & B & $\widetilde{B}$ & $B$ & 1 & 1 & 0 & 0 \\
\hline
\end{tabular}

Table 1. Field content of MSMSM. The column below F(B) denotes its fermionic (bosonic) content.

Once the field content is fixed, putting it all together the most generic renormalizable MW-SUSY Lagrangian of MSMSM, defined on the SMM $\left(S G_{N}\right)$, is now invariant under local gauge symmetry $S U_{c}(3) \otimes S U(2)_{L} \otimes U(1)$, where a set of gauge fields are coupled to various superfields among which is also Higgs supermultiplets. Furthermore, we especially separated from the rest the piece containing only the $\eta$-components of the particles defined on the supermanifold $S G_{\eta}$, which is important for the further discussion of a realistic realization of the MSMSM (next subsec.). Whereas, $\chi^{I}$ runs over all the particles, while $A^{J}$ runs over all the sparticles, the index $(a)$ labels the 3 different features in the gauge group, $V_{d}\left(H, H^{*}\right)$ is the scalar potential for each Higgs doublet $V_{u}\left(H_{u}, H_{u}{ }^{*}\right)=$ $-\frac{1}{2} m_{u}^{2}\left|H_{u}\right|^{2}+\frac{1}{4} \lambda_{u}^{2}\left|H_{u}\right|^{4} V_{d}\left(H_{d}, H_{d}{ }^{*}\right)=-\frac{1}{2} m_{d}^{2}\left|H_{d}\right|^{2}+\frac{1}{4} \lambda_{d}^{2}\left|H_{d}\right|^{4}$. A contribution of the " $D$ " term to the Higgs potential has also taken into account $V_{D}=\frac{1}{2} D^{(a)} D^{(a)}, \quad D^{(a)}=-g A^{I *} T_{I J}^{a} A^{J}$, or $V_{D}=\frac{g^{2}+g^{\prime 2}}{8}\left(\left|H_{u}\right|^{2}-\left|H_{d}\right|^{2} \bigoplus\right)^{2}+\frac{1}{2} g^{2}\left|H_{u} H_{d}{ }^{*}\right|^{2}$. The number of major free parameters in the Lagrangian are the primary coupling constants $\lambda_{Q}$ and $\lambda_{W}$ of nonlinear fermion interaction of the internal MW-components $i=Q, W$ and gauge couplings $g_{1}, g_{2}, g_{3}$. The SM relation $Q_{e}=g_{1} \cos \theta_{W}$ holds, where $\theta_{W}$ is the weak mixing angle $\cos ^{2} \theta_{W}=g^{2} /\left(g^{2}+g^{\prime 2}\right)$. The Yukawa couplings $\left(Y_{l} Y_{l}^{\prime}\right)$ are given: $Y=f_{Q}=Z_{Q}$. 


\subsection{Higgs doublets in MW-SUSY and Higgs mechanism}

Due to vacuum rearrangement in Q-world the Yukawa couplings arise between the fermion fields and corresponding isospinor-scalar $H$-mesons in conventional form. In the framework of suggested specific mechanism providing the effective attraction between the relativistic fermions caused by the exchange of the mediating induced gauge quanta in the $\mathrm{W}$-world, the two complex self-interacting isospinor-scalar Higgs doublets $\left(H_{u}, H_{d}\right)$ as well as their spin- $\frac{1}{2}$ SUSY partners $\left(\widetilde{H}_{u}, \widetilde{H}_{d}\right)$ Higgsinos arise as the Bose-condensate. Taking into account this slight difference from the MTSM arisen in the field content of SuMTSM in the Higgs sector the supersymmetric Lagrangian now also contains these fields coupled to the gauge fields in a gauge invariant way, when the symmetry-breaking Higgs bosons are counted off from the gap symmetry-restoring value as the point of origin.

The Higgs mechanism does work in the following way: Before the symmetry was broken in the $W$-world, the 2 complex $S U(2)_{L}$ Higgs doublets had 8 degrees of freedom. Three of them were the would-be Nambu-Goldstone bosons $G^{0}, G^{ \pm}$, which were absorbed to give rise the longitudinal modes of the massive $W$-components of the $Z^{0}$ and $W^{ \pm}$vector bosons, which simultaneously give rise the corresponding $x$ - components too, leaving 5 physical degrees of freedom. The latter consists of a charged Higgs boson pairs $H^{ \pm}$, a CP-odd neutral Higgs boson $A^{0}$, and CP-even neutral Higgs bosons $h^{0}$ and $H^{0}$. The mass eigenstates and would-be Nambu-Goldstone bosons are made of the original gauge-eigenstate fields, where the physical pseudoscalar Higgs boson $A^{0}$ is made of from the imaginary parts of $h_{u}^{0}$ and $h_{d}^{0}$, and is orthogonal to $G^{0}$; while the neutral scalar Higgs bosons are mixtures of the real parts of $h_{u}^{0}$ and $h_{d}^{0}$. The mass of any physical Higgs boson that is SM-like is strictly limited, as are the radiative corrections to the quartic potential terms. We calculated the tree-level masses for these Higgs states (sec.16) and shown that the $h^{0}$ Higgs boson arisen in the internal $W$-world is much heavier of that $Z^{0}$ boson. In contrast to the SM, the suggested microscopic approach predicts the electroweak symmetry breakdown in the $W$-world by the VEV of spin zero Higgs bosons and the transmission of electroweak symmetry breaking from the $W$-world to the $M_{4}$ spacetime continuum. The resulting Lagrangian of unified electroweak interactions of leptons and quarks ensues, which in lowest order approximation leads to the Lagrangian of phenomenological SM. In general, the self-energy operator underlies the Yukawa coupling constant, which takes into account a mass-spectrum of all expected collective excitations of bound quasi-particle pairs. If the MSM proves viable it becomes an crucial issue to hold in experiments the two testable predictions.

\subsection{Realistic realization of the MW-SUSY: M\$MSM}

The MW-SUSY cannot be an exact symmetry of nature and has to be realized in its broken phase. The major point of our strategy is a realistic realization of the supersymmetric extension of the MSM. Thus, the test of the theory will depend on its ability to account for the breaking of the MW-SUSY as well. Here, suggested approach creates a particular incentive for its study. In previous sections we have made a headway of reasonable framework of exact MW-SUSY defined on the exact MW-supermanifold $S G_{N}$. Therefore, one will be able to verify its virtues manifested, first of all, in the power of bosonfermion cancellations. One of the two principal offshoots of the supersymmetrization of the MSM is the solution of the zero point energy problem. Also, in its unbroken form it solves the technical aspects of the naturalness and hierarchy problem, when in non-SUSY theories scalar fields receive large mass corrections even if the bare mass is set to zero, and small masses are 'unnatural'. This applied to the Higgs bosons of the SM (as well as MSM) yields a difficulty in understanding of the smallness of $M_{Z}$ and how it can be kept stable against quantum corrections in some extensions of the SM containing apart from the weak scale $M_{Z}$ also a second larger scale $M_{G U T}>>M_{Z}$ [126,127], which holds in Grand Unified theories. The cancellation of quadratic divergences in SUSY theories is a consequence of general non-renormalization theorem, or the 'taming' of the quantum corrections, which stabilizes the Higgs mass and thus weak scale $M_{Z}$ without fine-tuning. It is remarkable that these attractive features of the unbroken MSMSM can be maintained as well in the broken M\$MSM. Achieving it one should perform an inverse passage $\left(S G_{\eta} \rightarrow G_{\eta}\right)$ to the $\$ \mathrm{MM}: \$ G_{N}$. It is due to the fact that the most powerful boson-fermion cancellation can be regarded as a direct consequence of a constraint stemming from holomorphy, therefore, it should be held even in the $M \$ M S M$. Then the Lagrangian $L_{\$ G_{N}}$ of 
the M\$MSM ensues from the Lagrangian $L_{S G_{N}}$ of the MSMSM: $L_{\phi G_{N}}=L_{S G_{N}}+L_{\text {soft }}$, where, one has $L_{s o f t}=\left(-m_{I J}^{2} A^{I} A^{J}-\frac{1}{2} \widetilde{m}_{a b} \lambda^{a} \lambda^{a}-\frac{1}{2} m_{u} \widetilde{H_{u}} \widetilde{H_{u}}-\frac{1}{2} m_{d} \widetilde{H_{d}} \widetilde{H_{d}}+\right.$ h.c. $)+b \epsilon_{i j}\left(H_{u}{ }^{i} H_{d}{ }^{j}+\right.$ h.c. $)$. Here $m_{I J}^{2}$ is the mass matrix for all the scalars of the chiral multiplets, $m \equiv\left(\widetilde{m}_{a b}, m_{u}, m_{d}\right)$ is the mass matrix respectively for the gauginos of each factor of the gauge group, and Higgsinos. The last term of the interaction is induced because these doublets above in free states imply $\hat{m}_{u}^{2}=-m_{u}^{2}+\lambda_{u}^{2} v_{u}^{2},=0, \hat{m}_{d}^{2}=$ $-m_{d}^{2}+\lambda_{d}^{2} v_{d}^{2}=0$, where $m^{2}, \lambda^{2}, v^{2}$ are respectively the mass, the coupling constant and VEV of given doublet. In the case at hand, certainly, there is an interaction between the bosons $H_{u}$ and $H_{d}$, when the strength of interaction $b$ will be fixed through the minimization conditions of the total Higgs potential. This can be used to derive a more physical relationship among the physical parameters. The case $\hat{m}_{u}^{2}=-\hat{m}_{d}^{2} \neq 0$ corresponds to the situation when the axion $A^{0}\left(m_{A^{0}}^{2}=0\right)$ has arisen after the breaking of electroweak gauge symmetry. But the other case of $\left(\hat{m}_{u}^{2}>0, \hat{m}_{d}^{2}>0\right)$ or $\left(\hat{m}_{u}^{2}<0, \quad \hat{m}_{d}^{2}<0\right)$, implies an existence of the neutral physical particle of the mass $m_{A^{0}}^{2}=\hat{m}_{u}^{2}+\hat{m}_{d}^{2} \neq 0$. Note that such Higgs doublets arisen on equal footing have counted off from the same point of origin for the same vacuum, then we will be interested physically in the most important simplest case when the electroweak symmetry breaking is parametrized just only by the single Higgs VEV $v_{u}=v_{d}, \quad \hat{m}_{u}^{2}=\hat{m}_{d}^{2}>0$. Of course, we shall carry out a computation in the generic case, but in the aftermath we shall turn to this case. The non-supersymmetric breaking terms do not spoil a condition of cancellation of quadratic divergences, i.e., a mass-squared sum rule $\operatorname{Str} M^{2} \equiv \sum_{J=0}^{1}(-1)^{2 J}(2 J+1) \operatorname{Tr} M_{J}^{2}=$ const. where $\vec{J}$ is the spin of the particles. It holds independently of the values of the scalar fields. Eventually the mass terms for the scalars contribute a constant, field independent piece, while a generic mass matrix for the fermions reads $M_{1 / 2}=M_{1 / 2}^{S}+\delta M_{1 / 2}$, where $M_{1 / 2}^{S}$ is the supersymmetric part of $M_{1 / 2}$, when $\delta M_{1 / 2}$ is given $\delta M_{1 / 2}=\left(\begin{array}{cc}\delta P_{I J} & \delta D_{I}^{b} \\ \delta D_{J}^{a} & \delta \widetilde{m}\end{array}\right)$. A computation for the considered fields gives $\delta P=0=\delta D$, while $\delta \widetilde{m}$ can be arbitrary.

\subsection{The viable SUSY-MTSM}

The realistic generating functional should be derived by passing back to the physical limit. Such a breaking of the MW-SUSY can be implemented by subtracting back all the explicit soft mass terms formerly introduced for the sparticles. These terms do not reintroduced the quadratic diagrams which motivated the introduction of SUSY framework. Therewith, the boson-fermion cancellation in the above-mentioned problems can be regarded as a consequence of a constraint stemming from holomorphy of the observables, therefore it will be held at the limit too. Thus, we extract the pertinent piece containing only the $\eta$-field components and then in afterwards pass to $M_{4}$ to get the final viable SUSY-MTSM (VMSM) yielding the realistic particle spectrum. Thus, if the VMSM proves viable it becomes an crucial issue to hold in the experiments at LEP2 and at the Tevatron three testable solid implications, which are drastically different from those of conventional MSSM models. The implication of quarks into the VMSM is carried out in the same way of leptons except that of appearance of quark mixing with Cabibbo angle and the existence of CP-violating complex phase in unitary matrix of quark mixing. The Q-components of the quarks contain at least one identical subquark, due to which the partial formfactors gain nonzero values. This underlies the quark mixing with Cabibbo angles. In lepton's case these formfactors are vanished and lepton mixing is absent. The CP-violation stems from the spanning. Adopting a simple viewpoint on Higgs sector the masses of leptons and quarks are obtained. We hope that the outlined VMSM, if it proves viable in the experiments at LEP2 and at the Tevatron, will be an attractive basis for the future theories. As yet no direct signal has been found in them, the absent of which has been cleared up the lower limits on Higgs bosons and sparticles masses. Furthermore, there is a tight upper limit $\left(m_{h^{0}}<150 \mathrm{GeV}\right)$ on the mass of the lightest Higgs boson $h^{0}$ among the 5 physical Higgs bosons predicted by the MSSM models. The current direct search limits from LEP2 give $m_{h^{0}}>75 \mathrm{GeV}$. Therefore, the future searches for this boson (if the mass is below 150 $\mathrm{GeV}$ or so) would be a crucial point in testing the efforts made in the conventional models building as well in the present MTSM based on a quite different approaches. The testable prediction of MTSM above together with a new one that the sparticles could never emerge in spacetime continuum since they have arisen only on the internal worlds, thus, they cannot be discovered in experiments nor at 
any energy range,

are the solid implications of the resulting VMSM for the experiments at LEP2, at the Tevatron and at LHC, which are drastically different from those of MSSM models. Which of these schemes, if any, is realized either exactly or at least approximately in nature remains to be seen in the years to come. For the theoretical apparatus of SUSY-MTSM, see (Ter-Kazarian, 2001c).

\subsection{Three solid testable predictions of VMSM}

Discussing now the relevance of our present approach to the physical realities we should attempt to provide some ground for checking the predictions of the VMSM against experimental evidence. It is remarkable that the resulting theory makes plausible following three testable implications for the current experiments, which are drastically different from the predictions of conventional models:

$(1,2)$. The two important phenomenological implication of the MSM, given above, just are the first two testable predictions of the VMSM for the current experiments.

3. It is well known that once $S U(2)_{L} \otimes U(1)_{Y}$ is broken, the fields with different $S U(2)_{L} \otimes$ $U(1)_{Y}$ quantum numbers can mix if they have the same $S U(3)_{c} \otimes U(1)_{e m}$ quantum numbers. Such a phenomenon occurs in the sfermion sector of the M\$MSM. If one ignore mixing between sfermions of different generations but will include the mixing between $S U(2)$ doublet and singlet sfermions then the sfermion mass matrix decomposes into a series of $2 \times 2$ matrices of the sfermions of a given flavour. The charginos are mixtures of the charged Higgsinos and the charged gauginos, and neutralinos are the mixture of neutral Higgsinos and the neutral gauginos, etc.. We can readily obtain the resulting explicit forms of corresponding mass matrices within standard technique. But shall forbear to write them out here as the sfermions are no longer of consequence for discussion of the final fields defined on $M_{4}$. The sparticles could never emerge in $M_{4}$ and will be of no interest for the future experiments. By this we arrived to the second principle point of drastic deviation of M\$MSM from the conventional MSSM models. In MSSM models as well in any conventional SUSY theory the supersymmetry was implemented in the Minkowski space $M_{4}$ by adding a new four odd dimensions, and there are two major motivation for SUSY to be realized in the TeV range, i.e., the masses of sparticles are of the order of a few $\mathrm{TeV}$ or less. First one is a solution of the hierarchy problem, when in order to introduce no new fine-tunning all soft terms should be of the same order of magnitude at most in the TeV range-weak scale. The second motivation for low energy SUSY comes from the view point of gauge unification (a supersymmetric GUT). Since the current experiments will explore this energy range, then, the second great expectation of such theories arise that at least some of the sparticles can be found and their parameters like masses and coupling constants will also be measured (the precise measurements). Reflecting upon the results far obtained here, in a strong contrast to such theories the unbroken MW-SUSY is implemented on the MW-SMM: $S G_{N}$ by, at first, lifting up $G_{\eta} \rightarrow S G_{\eta}$ and consequently making an inverse passage to the $\$ G_{N}\left(S G_{\eta} \rightarrow G_{\eta}\right)$ on which the resulting theory MSMSM is defined. Applying the final passage $\left(G_{\eta} \rightarrow M_{4}\right)$ we arrive to the final VMSM, where only the particles will survive on the $M_{4}$ at the real physical limit under the R-parity conservation. Thus,

- all the sparticles never could emerge in the $M_{4}$ neither at Te $V$ range nor at any energy range at all.

From the view point of achieving the final potentially realistic supersymmetric field theory this will be third crucial test in experiments above for verifying the efforts made either in MSSM model building (the conventional SUSY theories) or in suggested VMSM (the MW-SUSY), which are based on two quite different approaches. To sum up the discussion thus far, we have argued that, in strong contrast to conventional SUSY theories, if the VMSM given here proves viable it becomes an crucial issue to hold in experiments the above-mentioned three tests.

\subsection{A brief outlook}

Let us give a brief outlook on the key points of physical picture described in this subsection. We derive the MW-SUSY, which has an algebraic origin in the sense that it has arisen from the subquark algebra defined on the internal worlds, while the nilpotent supercharge operators are derived. Therefore, the MW-SUSY realized only on the internal worlds but not on the spacetime continuum. 
Thus, it cannot be an exact symmetry of nature and has to be realized in its broken phase. Our purpose above is much easier to handle, by restoring in the first the 'exact' MW-SUSY. It can be achieved by lifting up each sparticle to corresponding particle state. This enables the sparticle to be included in the same supermultiplet with corresponding particle. Due to different features of particles and sparticles when passing back to physically realistic limit, one must have always to distinguish them by introducing an additional discrete internal symmetry, i.e., the multiplicative $Z_{2} \quad R$-parity.

We write then the most generic renormalizable MW-SUSY action involving gauge and supersymmetric matter frame fields, and, thus, the corresponding generating functional. Therein, we are led to the principal point of drastic change of the standard SUSY scheme to specialize the superpotential to be in such a form, which enables the microscopic approach to the key problems of particle phenomenology.

The realistic generating functional should be derived by passing back to the physical limit. Such a breaking of the MW-SUSY can be implemented by subtracting back all the explicit soft mass terms formerly introduced for the sparticles. These terms do not reintroduced the quadratic diagrams which motivated the introduction of SUSY framework. Therewith, the boson-fermion cancellation in the above-mentioned problems can be regarded as a consequence of a constraint stemming from holomorphy of the observables, therefore it will be held at the realistic physical limit too. Thus, we extract the pertinent piece containing only the $\eta$-field components and then in afterwards pass to $M_{4}$ to get the final VMSM yielding the realistic particle spectrum.

Thus, if the VMSM proves viable it becomes an crucial issue to hold in the current experiments three testable solid implications, which are drastically different from those of conventional MSSM models.

The implication of quarks into the VMSM is carried out in the same way of leptons except that of appearance of quark mixing with Cabibbo angle and the existence of CP-violating complex phase in unitary matrix of quark mixing. The Q-components of the quarks contain at least one identical subquark, due to which the partial formfactors gain nonzero values. This underlies the quark mixing with Cabibbo angles. In lepton's case these formfactors are vanished and lepton mixing is absent. The CP-violation stems from the spanning. Adopting a simple viewpoint on Higgs sector the masses of leptons and quarks are given.

We hope that the outlined VMSM, if it proves viable in the current experiments, will be an attractive basis for the future theories. As yet no direct signal has been found in them, the absent of which has been cleared up the lower limits on Higgs bosons and sparticles masses.

\section{Spacetime deformation induced inertia effects}

The principle of inertia, whose origin can be traced back to the works developed by Galileo and Newton, is one of the fundamental principles of the classical mechanics. This governs the uniform motion of a body and describes how it is affected by applied forces. The universality of gravitation and inertia attribute to the geometry but as having a different natures. However, despite the advocated success of general relativity (GR), the problem of inertia stood open and that this is still an unknown exciting problem to be challenged. The inertia effects cannot be in full generality identified with gravity within GR as it was proposed by Einstein in 1918, because there are many experimental controversies to question the validity of such a description, for details see e.g. (Ter-Kazarian, 2012) and references therein. The universality of the gravitation and inertia attributes to the weak principle of equivalence (WPE), which establishes the independence of free-fall trajectories of the internal composition and structure of bodies. Currently, the observations performed in the Earth-Moon-Sun system, or at galactic and cosmological scales, make it possible to probe the fundamental issue of gravitation/inertia more deeply by imposing the constraints of various analyses. The inertia effects in fact are of vital interest also for the phenomenological aspects of the long-standing problem of neutrino oscillations. All this variety has evoked the study of the inertial effects in an accelerated and rotated frame of stationary laboratories on Earth, relative to the local inertial frames. The standard extension of Lorentz invariance to accelerated observers in Minkowski spacetime is based on the hypothesis of locality, which in effect replaces the accelerated observer by a continuous infinity of 
hypothetical momentarily comoving inertial observers along its wordline. This assumption, as well as its restricted version, so-called, clock hypothesis, which is a hypothesis of locality only concerned about the measurement of time, are reasonable only if the curvature of the wordline could be ignored. This question has become a major preoccupation of physicists. As long as all relevant length scales in feasible experiments are very small in relation to the huge acceleration lengths of the tiny accelerations we usually experience, the differences between observations by accelerated and comoving inertial observers will also be very small. However this works out, it is still reminds us of a puzzling underlying reality of the phenomenon of inertia. Beyond the WPE, there is nothing convincing in the basic postulates of physics for the origin and nature of inertia to decide on the issue.

On the other hand it seems that the inertia effects display no any physical characteristics of gravitation, because there are important reasons to question the validity of such a description. For example, there are a few experiments which tested the key question of anisotropy of inertia stemming from the idea that the matter in our galaxy is not distributed isotropically with respect to the earth, and hence if the inertia is due to gravitational interactions, then the inertial mass of a body will depend on the direction of its acceleration with respect to the direction towards the center of our galaxy. If the nuclear structure of $L i^{7}$ is treated as a single $P_{3 / 2}$ proton in a central nuclear potential, the variation $\Delta m$ of mass with direction, if it exists, was found to satisfy $\frac{\Delta m}{m} \leq 10^{-20}$. This proves that there is no anisotropy of mass which is due to the effects of mass in our galaxy. Moreover, unlike gravitation, a curvature arisen due to acceleration of coordinate frame of interest, i.e. a `fictitious gravitation' which can be globally removed by appropriate coordinate transformations, relates to this coordinate system itself and does not affect at once all the other systems or matter fields. Despite our best efforts, all attempts to obtain a true knowledge of the inertial effects and the geometry related to the noninertial reference frames of an arbitrary observer seem doomed, unless we find a physical principle the inertia might refer to, and construct the relativistic theory of inertia. Otherwise one wanders in a darkness.

It is the purpose of present section to carry out some details of the program of spacetime deformation theory to probe the origin and nature of the phenomenon of inertia. We ascribe the inertia effects to the geometry itself but as having a nature other than gravitation. To this aim, we propose a hypothetical space-companion to every particle. We explore the 2D, so-called, master-space (MS), subject to certain rules. The MS, embedded in the background $4 \mathrm{D}$-space, is an indispensable individual companion to the particle of interest, without relation to the other matter. This notion is quite intuitive; in essence, it says that the particle apparently just has to live with MS-companion as an intrinsic property. This together with the heuristic idea that the inertia effects arise as a deformation/(distortion of local internal properties) of MS, are the highlights of the present paper. We will be brief and often ruthlessly suppress the indices without notice. Unless otherwise stated we take natural units, $h=c=1$.

\subsection{The hypothetical flat MS-companion: a toy model}

As a preliminary step we now conceive of two different spaces, one would be $4 \mathrm{D}$ background Minkowski space, $M_{4}$, and another one should be MS embedded in the $M_{4}$, which is an indispensable individual companion to the particle, without relation to the other matter. The flat MS in suggested model is assumed to be 2D Minkowski space, $M_{2}: M_{2}=R_{(+)}^{1} \oplus R_{(-)}^{1}$. The ingredient 1D-space $R_{A}^{1}$ is spanned by the coordinates $\eta^{A}$, where we use the naked capital Latin letters $A, B, \ldots=( \pm)$ to denote the world indices related to $M_{2}$. The metric in $M_{2}$ is $\bar{g}=\bar{g}\left(\bar{e}_{A}, \bar{e}_{B}\right) \bar{\vartheta}^{A} \otimes \bar{\vartheta}^{B}$, where $\bar{\vartheta}^{A}=d \eta^{A}$ is the infinitesimal displacement. The basis $\bar{e}_{A}$ at the point of interest in $M_{2}$ consists of two real null vectors: $\bar{g}\left(\bar{e}_{A}, \bar{e}_{B}\right) \equiv<\bar{e}_{A}, \bar{e}_{B}>={ }^{*} o_{A B}$. The norm, $i \bar{d} \equiv d \hat{\eta}$, given in this basis reads $i \bar{d}=\bar{e} \bar{\vartheta}=\bar{e}_{A} \otimes \bar{\vartheta}^{A}$, where $i \bar{d}$ is the tautological tensor field of type $(1,1), \bar{e}$ is a shorthand for the collection of the 2tuplet $\left(\bar{e}_{(+)}, \bar{e}_{(-)}\right)$. We may equivalently use a temporal $q^{0} \in T^{1}$ and a spatial $q^{1} \in R^{1}$ variables $q^{r}\left(q^{0}, q^{1}\right)(r=0,1)$, such that $M_{2}=R^{1} \oplus T^{1}$. The norm, $i \bar{d}$, now can be rewritten in terms of displacement, $d q^{r}$, as $i \bar{d}=d \hat{q}=e_{0} \otimes d q^{0}+e_{1} \otimes d q^{1}$, where $e_{0}$ and $e_{1}$ are, respectively, the temporal and spatial basis vectors. Then, a smooth map $f: M_{2} \rightarrow M_{4}$ is defined to be an immersion - an embedding is a function that is a homeomorphism onto its image. In fact, we assume the particle has 
to be moving simultaneously in the parallel individual $M_{2}$ space and the ordinary $4 \mathrm{D}$ background space (either Minkowskian or Riemannian). Let the non-accelerated observer uses the inertial coordinate frame $S_{(2)}$ for the position $q^{r}$ of a free test particle in the flat $M_{2}$. We may choose the system $S_{(2)}$ in such a way as the time axis $e_{0}$ lies along the time axis of a comoving inertial frame $S_{4}$, such that the time coordinates in the two systems are taken the same, $q^{0}=t$. Hence, given the inertial frames $S_{(4)}, S_{(4)}^{\prime}, S_{(4)}^{\prime \prime}, \ldots$ in the $M_{4}$, in this manner we may define the corresponding inertial frames $S_{(2)}$, $S_{(2)}^{\prime}, S_{(2)}^{\prime \prime}, \ldots$ in the $M_{2}$. Continuing on our quest, we next define the concepts of absolute and relative states of the ingredient spaces $R_{A}^{1}$. The measure for these states is the very magnitude of the velocity components $v^{A}$ of the particle: Definition: The ingredient space $R_{A}^{1}$ of the individual MS-companion of the particle is said to be in absolute (abs) state if $v^{A}=0$, and in relative (rel) state if $v^{A} \neq 0$. Therefore, the MS can be realized either in the semi-absolute state (rel, abs), or (abs, rel), or in the total relative state (rel, rel). It is remarkable that the total-absolute state, (abs, abs), which is equivalent to the unobservable Newtonian absolute two-dimensional spacetime, cannot be realized because of the relation $v^{(+)}+v^{(-)}=\sqrt{2}$. An existence of the absolute state of the $R_{A}^{1}$ is an immediate cause of the light traveling in empty space $R^{1}$ along the $q$-axis with a maximal velocity $v_{q}=c$ (we re-instate the factor (c)) in the $(+)$-direction corresponding to the state $\left(v^{(+)}, 0\right) \Leftrightarrow$ (rel, abs), and in the $(-)$-direction corresponding to the state $\left(0, v^{(-)}\right) \Leftrightarrow\left(\right.$ abs, rel). The absolute state of $R_{A}^{1}$ manifests its absolute character in the important for SR fact that the resulting velocity of light in the empty space $R^{1}$ is the same in all inertial frames $S_{(2)}, S_{(2)}^{\prime}, S_{(2)}^{\prime \prime}, \ldots$, i.e., in empty space light propagates independently of the state of motion of the source - if $v^{A}=0$ then $v^{A \prime}=v^{A \prime \prime}=\ldots=0$. This observation allows us to lay forth the RLI-Conjecture: The non-zero local rate $\varrho(\eta, m, f)$ of instantaneously change of a constant velocity $v^{A}$ (both magnitude and direction) of a massive $(m)$ test particle under the unbalanced net force $(\vec{f})$ is the immediate cause of a deformation/(distortion of the local internal properties) of MS: $M_{2} \rightarrow \widetilde{\mathcal{M}}_{2}$. The MS-companion is not measurable directly, but in going into practical details, we will determine the function $\varrho(\eta, m, \vec{f})$ and show that a deformation/(distortion of local internal properties) of MS is the origin of inertia effects that can be observed by us.

\subsection{Model building in the 4D background Minkowski spacetime}

At first, we construct the RTI when the relativistic test particle accelerated in the Minkowski $4 \mathrm{D}$ background flat space, $M_{4}$, under an unbalanced net force other than gravitational. It proves necessary to provide, further, a constitutive ansatz of simple, yet tentative, linear distortion transformations, which, according to RLI-Conjecture, can be written in terms of local rate $\varrho(\eta, m, f)$ of instantaneously change of the measure $v^{A}$ of massive $(m)$ test particle under the unbalanced net force $(f): e_{(\tilde{+})}(\varrho)=D_{(\tilde{+})}^{B}(\varrho) \bar{e}_{B}=\bar{e}_{(+)}-\varrho(\eta, m, f) v^{(-)} \bar{e}_{(-)}$, and $e_{(\tilde{-})}(\varrho)=D_{(\tilde{-})}^{D}(\varrho) \bar{e}_{B}=$ $\bar{e}_{(-)}+\varrho(\eta, m, f) v^{(+)} \bar{e}_{(+)}$. Clearly, these transformations imply a violation of the relation $e_{\tilde{A}}^{2}(\varrho) \neq 0$ for the null vectors $\bar{e}_{A}$. We parameterize the deformation tensor $\Omega_{B}{ }^{A}$ in terms of the parameters $\tau_{1}$ and $\tau_{2}$ as $\Omega_{(+)}^{(+)}=\Omega_{(-)}^{(-)}=\tau_{1}\left(1+\tau_{2} \varrho^{2}\right), \quad \Omega_{(+)}^{(-)}=-\tau_{1}\left(1-\tau_{2}\right) \varrho v^{(-)}, \quad \Omega_{(-)}^{(+)}=\tau_{1}\left(1-\tau_{2}\right) \varrho v^{(+)}$, where $\bar{\varrho}^{2}=v^{2} \varrho^{2}, \quad v^{2}=v^{(+)} v^{(-)}=1 / 2 \gamma_{q}^{2}$ and $\gamma_{q}=\left(1-v_{q}^{2}\right)^{-1 / 2}$. Suppose a second observer, who makes measurements using a frame of reference $\widetilde{S}_{(2)}$ which is held stationary in deformed/distorted space $\widetilde{\mathcal{M}}_{2}$, uses for the test particle the corresponding spacetime coordinates $\widetilde{q}^{\tilde{r}}\left(\left(\widetilde{q}^{\tilde{0}}, \widetilde{q}^{\tilde{1}}\right) \equiv(\widetilde{t}, \widetilde{q})\right)$. This gives the general transformation equations for spatial and temporal coordinates as follows $\left(\vec{e}_{q} \equiv e_{1}, q \equiv q^{1}\right)$ : $d \widetilde{t}=\tau_{1} d t, \quad d \widetilde{q}=\tau_{1}\left[d q\left(1+\frac{\tau_{2} \varrho v_{q}}{\sqrt{2}}\right)-\frac{\tau_{2} \varrho}{\sqrt{2}} d t\right]=\tau_{1}\left(d q-\frac{\tau_{2} \varrho}{\sqrt{2} \gamma_{q}^{2}} d t\right)$. The difference of the vector, $d \hat{q} \in M_{2}$, and the vector, $\widetilde{d \hat{q}} \in \widetilde{\mathcal{M}}_{2}$, can be interpreted by the second observer as being due to the deformation/distortion of flat space $M_{2}$. However, this difference with equal justice can be interpreted by him as a definite criterion for the absolute character of his own state of acceleration in $M_{2}$, rather than to any absolute quality of a deformation/distortion of $M_{2}$. To prove this assertion, note that the transformation equations give a reasonable change at low velocities $v_{q} \simeq 0$, as $d \widetilde{t}=\tau_{1} d t, \quad d \widetilde{q} \simeq \tau_{1}\left(d q-\frac{\tau_{2} \varrho}{\sqrt{2}} d t\right)$. This becomes conventional transformation equations to accelerated $\left(a_{\text {net }} \neq 0\right)$ axes if we assume $d\left(\tau_{2} \varrho\right) / \sqrt{2} d t=a_{\text {net }}$ and $\tau_{1}\left(v_{q} \simeq 0\right)=1$, where $a_{\text {net }}$ is a magnitude of proper net acceleration. In high velocity limit $v_{q} \simeq 1, \bar{\varrho} \simeq 0,\left(d \eta^{(-)}=v^{(-)} d t \simeq 0, v^{(+)} \simeq v \simeq \sqrt{2}\right)$, we have $d \widetilde{t}=\tau_{1} d t \simeq \tau_{1} d q \simeq d \widetilde{q}$. 
To this end, the inertial effects become zero. Let $\vec{a}_{\text {net }}$ be a local net 3 -acceleration of an arbitrary observer with proper linear 3-acceleration $\vec{a}$ and proper 3-angular velocity $\vec{\omega}$ measured in the rest frame: $\vec{a}_{n e t}=\frac{d \vec{u}}{d s}=\vec{a} \wedge \vec{u}+\vec{\omega} \times \vec{u}$, where $\mathbf{u}$ is the 4-velocity. A magnitude of $\vec{a}_{n e t}$ can be computed as the simple invariant of the absolute value $\left|\frac{d \mathbf{u}}{d s}\right|$ as measured in rest frame: $|\mathbf{a}|=\left|\frac{d \mathbf{u}}{d s}\right|=\left(\frac{d u^{l}}{d s}, \frac{d u_{l}}{d s}\right)^{1 / 2}$. We may introduce the very concept of the local absolute acceleration (in Newton's terminology) brought about via the Fermi-Walker transported frames as $\vec{a}_{a b s} \equiv \vec{e}_{q} \frac{d\left(\tau_{2} \varrho\right)}{\sqrt{2} d s_{q}}=\vec{e}_{q}\left|\frac{d e_{\hat{0}}}{d s}\right|=\vec{e}_{q}|\mathbf{a}|$, where we choose the system $S_{(2)}$ in such a way as the axis $\vec{e}_{q}$ lies along the net 3 -acceleration $\left(\vec{e}_{q} \| \vec{e}_{a}\right), \quad\left(\vec{e}_{a}=\vec{a}_{n e t} /\left|\vec{a}_{n e t}\right|\right)$. Hereinafter, we may simplify the flat-deformation tensor $\Omega_{A}{ }^{B}$ by setting $\tau_{2}=1$, such that $\Omega_{(+)}^{(+)}=$ $\Omega_{(-)}^{(-)} \equiv \Omega(\bar{\varrho})=1+\bar{\varrho}^{2}, \quad \Omega_{(+)}^{(-)}=\Omega_{(-)}^{(+)}=0$, and the general metric in $\widetilde{\mathcal{M}}_{2}$ reads $d \widetilde{s}_{q}^{2}=\Omega^{2}(\bar{\varrho}) d s_{q}^{2}$. Hence $\varrho=\sqrt{2} \int_{0}^{s_{q}}|\mathbf{a}| d s_{q}^{\prime}$. Then we obtain the key relation between a so-called inertial acceleration, arisen due to the curvature of MS, $\vec{a}_{i n}=\vec{e}_{a} a_{i n}, \quad a_{i n}=\frac{d^{2} \widetilde{q}}{d \widetilde{s}_{q}^{2}}=-\Gamma_{\tilde{r} \tilde{s}}^{1}(\varrho) \frac{d \widetilde{q}^{\tilde{r}}}{d \widetilde{s}_{q}} \frac{d \widetilde{q}^{\tilde{s}}}{d \tilde{s}_{q}}=\frac{1}{\sqrt{2}}\left(\frac{d^{2} \widetilde{\eta}^{(+)}}{d \widetilde{s}_{q}^{2}}-\frac{d^{2} \widetilde{\eta}^{(-)}}{d \widetilde{s}_{q}^{2}}\right)$, and a local absolute acceleration as follows: $\Omega^{2}(\bar{\varrho}) \gamma_{q} \vec{a}_{i n}=-\vec{a}_{a b s}$, where $\Gamma_{\tilde{r} \tilde{s}}^{1}(\varrho)$ are the Christoffel symbols constructed by the metric. This provides a quantitative means for the inertial force $\vec{f}_{(i n)}$ : $\vec{f}_{(i n)}=m \vec{a}_{i n}=-m \Gamma_{\tilde{r} \tilde{s}}^{1}(\varrho) \frac{d \widetilde{q}^{\tilde{r}}}{d \bar{s}_{q}} \frac{d \widetilde{q}^{\tilde{s}}}{\bar{s}_{q}}=-\frac{m \vec{a}_{a b s}}{\Omega^{2}(\bar{\varrho}) \gamma_{q}}$. Thus, it takes force to disturb an inertia state, i.e. to make the absolute acceleration $\left(\vec{a}_{a b s} \neq 0\right)$. The absolute acceleration is due to the real deformation/distortion of the space $M_{2}$. The relative $\left(d\left(\tau_{2} \varrho\right) / d s_{q}=0\right)$ acceleration (in Newton's terminology) (both magnitude and direction), to the contrary, has nothing to do with the deformation/distortion of the space $M_{2}$ and, thus, it cannot produce an inertia effects.

\subsection{Beyond the hypothesis of locality}

The hypothesis of locality represents strict restrictions, because in other words, it approximately replaces a noninertial frame of reference $\widetilde{S}_{(2)}$, which is held stationary in the deformed/distorted space $\widetilde{\mathcal{M}}_{2} \equiv V_{2}^{(\varrho)}(\varrho \neq 0)$, with a continuous infinity set of the inertial frames $\left\{S_{(2)}, S_{(2)}^{\prime}, S_{(2)}^{\prime \prime}, \ldots\right\}$ given in the flat $M_{2}(\varrho=0)$. In this situation the use of the hypothesis of locality is physically unjustifiable. Therefore, it is worthwhile to go beyond the hypothesis of locality with special emphasis on distortion of MS, which we might expect will essentially improve the standard results. Therefore, our strategy now is to deform the metric by carrying out an additional deformation of semi-Riemannian $4 \mathrm{D}$ background space $V_{4}^{(0)} \rightarrow \widetilde{\mathcal{M}}_{4} \equiv V_{4}^{(\varrho)}$, which, as a corollary, will recover the complete metric $g \quad(\varrho \neq 0)$ of the distorted MS - $V_{2}^{(\varrho)}$. The resulting deformed metric of the space $V_{4}^{(\varrho)}$ can be split as $g_{\mu \nu}(\varrho)=\Upsilon^{2}(\varrho) \breve{g}_{\mu \nu}+\gamma_{\mu \nu}(\varrho)$, provided $\gamma_{\mu \nu}(\varrho)=\left[\gamma_{\hat{a} \hat{b}}-\Upsilon^{2}(\varrho) o_{\hat{a} \hat{b}}\right] \breve{e}_{\mu}^{\hat{a}}{ }_{\mu}{ }^{\hat{b}}{ }_{\nu}, \quad \gamma_{\hat{c} \hat{d}}=o_{\hat{a} \hat{b}} \pi_{\hat{c}}{ }^{\hat{a}} \pi_{\hat{d}}^{\hat{b}}$, where $\Upsilon(\varrho)=\pi_{\hat{a}}^{\hat{a}}(\varrho)$ and $\gamma_{\hat{a} \hat{b}}(\breve{x})$ are the second deformation matrices. Hence, in general, the metric $g(\varrho)$ is decomposed in the form $g(\varrho)=\pi^{2}(\varrho) \breve{g}+\gamma(\varrho)$, where $\gamma(\varrho)=\gamma_{\mu \nu}(\varrho) d \breve{x}^{\mu} \otimes d \breve{x}^{\nu}$ and $\Upsilon(\varrho)=\pi_{\hat{a}}^{\hat{a}}(\varrho)=\pi(\varrho)$. A generalized transport for deformed frame $e_{\hat{a}}$, which includes both the Fermi-Walker transport and distortion of MS, can be written in the form $\frac{d e_{\hat{a}}^{\mu}}{d s}=\widetilde{\Phi}_{a}{ }^{b} e_{\hat{b}}^{\mu}$, where a deformed acceleration tensor $\widetilde{\Phi}_{a}{ }^{b}$ concisely is given by $\widetilde{\Phi}=(d \ln \pi / d s)+\pi \Phi \pi^{-1}$.

\subsection{Involving the background semi-Riemann space $V_{4}$}

We can always choose natural coordinates $X^{\alpha}(T, X, Y, Z)=(T, \vec{X})$ with respect to the axes of the local free-fall coordinate frame $S_{4}^{(l)}$ in an immediate neighbourhood of any spacetime point $\left(\breve{x}_{p}\right) \in$ $V_{4}$ in question of the background semi- Riemann space, $V_{4}$, over a differential region taken small enough so that we can neglect the spatial and temporal variations of gravity for the range involved. The values of the metric tensor $\breve{g}_{\mu \nu}$ and the affine connection $\breve{\Gamma}_{\mu \nu}^{\lambda}$ at the point $\left(\breve{x}_{p}\right)$ are necessarily sufficient information for determination of the natural coordinates $X^{\alpha}\left(\breve{x}^{\mu}\right)$ in the small region of the neighbourhood of the selected point. Then the whole scheme outlined above should hold in the frame $S_{4}^{(l)}$. The relativistic gravitational force $\breve{f}_{g}^{\mu}(\breve{x})$ exerted on the test particle of the mass $(m)$ is given by $\breve{f}_{g}^{\mu}(\breve{x})=m \frac{d^{2} \breve{x}^{\mu}}{d \breve{s}^{2}}=-m \breve{\Gamma}_{\nu \lambda}^{\mu}(a) \frac{d \breve{x}^{\nu}}{d \breve{s}} \frac{d \breve{x}^{\lambda}}{d \breve{s}}$. The frame $S_{4}^{(l)}$ will be valid if only the gravitational force given in this coordinate frame $f_{g(l)}^{\alpha}=\frac{\partial X^{\alpha}}{\partial \widetilde{x}^{\mu}} f_{g}^{\mu}$, could be removed by the inertial force. Whereas, as before, the two systems $S_{2}$ and $S_{4}^{(l)}$ can be chosen in such a way as the axis $\vec{e}_{q}$ of $S_{(2)}$ lies $\left(\vec{e}_{q}=\vec{e}_{f}\right)$ along the 
A new look at some aspects of geometry, particle physics, inertia, radiation and cosmology

acting net force $\vec{f}=\vec{f}_{(l)}+\vec{f}_{g(l)}$, where $\vec{f}_{(l)}$ is the SR value of the unbalanced relativistic force other than gravitational in the frame $S_{4}^{(l)}$, while the time coordinates in the two systems are taken the same, $q^{0}=t=X^{0}=T$. We now may write $\frac{1}{\sqrt{2}} \frac{d\left(\tau_{2} \varrho\right)}{d s_{q}}=\frac{1}{m}\left|f_{(l)}^{\alpha}+f_{g(l)}^{\alpha}\right|$, such that the general inertial force reads $\breve{\vec{f}}_{(i n)}=m \vec{a}_{i n}=-\frac{m \vec{a}_{a b s}}{\Omega^{2}(\bar{\varrho}) \gamma_{q}}=-\frac{\vec{e}_{f}}{\Omega^{2}(\bar{\varrho}) \gamma_{q}}\left|f_{(l)}^{\alpha}-m \frac{\partial X^{\alpha}}{\partial \breve{x}^{\sigma}} \breve{\Gamma}_{\mu \nu}^{\sigma} \frac{d \breve{x}^{\mu}}{d S} \frac{d \breve{x}^{\nu}}{d S}\right|$. Despite of totally different and independent sources of gravitation and inertia, at $f_{(l)}^{\alpha}=0$, this establishes independence of free-fall $\left(v_{q}=0\right)$ trajectories of the mass, internal composition and structure of bodies. This furnishes a justification for the introduction of the WPE. A remarkable feature is that, although the inertial force has a nature different than the gravitational force, nevertheless both are due to a distortion of the local inertial properties of, respectively, 2D MS and 4D-background space.

\subsection{The principle of equivalence in the $\mathrm{RC}$ space}

The RC manifold, $U_{4}$, is a particular case of general metric-affine manifold $\widetilde{M}_{4}$, restricted by the metricity condition $N_{a b}=0$, when a nonsymmetric linear connection, $\Gamma$, is said to be metric compatible. To avoid any possibility of confusion, here and throughout we again use the first half of Latin alphabet $(a, b, c, \ldots=0,1,2,3$ rather than $( \pm))$ now to denote the anholonomic indices referred to the tangent space, which is endowed with the Lorentzian metric $o_{a b}:=\operatorname{diag}(+---)$. The space, $U_{4}$, also locally has the structure of $M_{4}$. In the case of the RC space there also exist orthonormal reference frames which realize an 'anholonomic' free-fall elevator. That is, for any single point $P \in U_{4}$, there exist coordinates $\left\{x^{\mu}\right\}$ and an orthonormal frame $\left\{e_{a}\right\}$ in a neighborhood of $P$ such that $e_{a}=\delta_{a}^{\mu} \partial_{x^{\mu}}$, and $\Gamma_{a}^{b}=0$ at $P$, where $\Gamma_{a}^{b}$ are the connection 1 -forms referred to the frame $\left\{e_{a}\right\}$. Therefore the existence of torsion does not violate the PE. Suppose that we have a tetrad $\left\{e_{a}(x)\right\}$ at the point $P$, and a tetrad $\left\{e_{a}(x+d x)\right\}$ at another point in a neighbourhood of $P$; then, we can apply a suitable Lorentz rotation to $e_{a}(x+d x)$, so that it becomes parallel to $e_{a}(x)$. Given a vector $v$ at $P$, it follows that the components $v_{c}=v \cdot e_{c}$ do not change under parallel transport from $x$ to $x+d x$, provided the metricity condition holds. Hence, the connection coefficients $\omega^{a b}{ }_{\mu}(x)$ at $P$, defined with respect to this particular tetrad field, vanish: $\omega^{a b}{ }_{\mu}(P)=0$. This property is compatible with $g_{a b}^{\prime}=o_{a b}$, since Lorentz rotation does not influence the value of the metric at a given point. In more general geometries, where the symmetry of the tangent space is higher than the Poincare group, the usual form of the PE is violated and local physics differs from SR. Taking this into account, we derive a general expression of the relativistic inertial force exerted on the extended spinning body moving in the Rieman-Cartan space (Ter-Kazarian, 2012).

\section{Probing the inertia behind SUSY}

The model discussed in previous section illustrates the problems of inertia effects described in the framework of classical physics, but it also hints at a possible complete solution. We will use this model as a backdrop to explore first the SLC in a new perspective of rigid double transformations of, so-called, master space-induced supersymmetry (MS-SUSY), subject to certain rules (Ter-Kazarian, 2013b). The theories with extended $N_{\max }=4$ supersymmetries, namely $N=4$ super-Yang-Mills theories, if only such symmetries are fundamental to nature, lead to the model of ELC in case of the apparent violations of SLC, the possible manifestations of which arise in a similar way in all particle sectors. We show that in the ELC-framework the propagation of the superluminal particle could be consistent with causality, and give a justification of forbiddance of Vavilov-Cherenkov radiation/or analog processes in vacuum. However, we must be careful about the physical relevance of the standard theory of extended supersymmetry which does not allow for chiral fermions, and that its spectrum in no way resembles that of the observed in nature. Consequently, in the framework of local MSSUSY, we address the accelerated motion, while, unlike gravitation, a curvature of space-time now arises entirely due to the inertial properties of the Lorentz-rotated frame of interest, i.e. a fictitious gravitation which can be globally removed by appropriate coordinate transformations. The only source of graviton and gravitino, therefore, is the acceleration of a particle. 


\subsection{A background 'motion' space $\underline{M}$}

With regard now to our original question as to the understanding of the physical processes that underly the motion, we tackle the problem in the framework of quantum field theory (Ter-Kazarian, 2013b). Let us consider functional integrals for a quantum-mechanical system with one degree of freedom. Denote by $x(t)$ the position operator in the Heisenberg picture, and by $\mid x, t>$ its eigenstates. The probability amplitude that a particle which was at $x$ at time $t$ will be at point $x^{\prime}$ at time $t^{\prime}$, also called the Schwinger transformation function for these points, is $F\left(x^{\prime} t^{\prime} ; x t\right)=\left\langle x^{\prime} t^{\prime}\right| x t>$. For a particle moving through the two infinitesimally closed points of original space, this in somehow or other implies the elementary act consisting of the annihilation of a particle at the point $x$ and time $t$ and, subsequently, its creation at the point $x^{\prime}$ and time $t^{\prime}$. The particle can move with different velocities which indicates to existence of the intermediate, so-called, motion state. Then the annihilation of a particle at point $x$ and time $t$ can intuitively be understood as the transition from the initial state $\mid x, t>$ to the intermediate motion state, $\mid \underline{x}, \underline{t}>$, yet unknown, where $\underline{x}(\underline{t})$ represent atomic element of idealized motion point event. Meanwhile, the creation of a particle at infinitesimally closed final point $x^{\prime}$ and time $t^{\prime}$ means the subsequent transition from the intermediate motion state, $\mid \underline{x}, \underline{t}>$, to the final state, $\mid x^{\prime}, t^{\prime}>$. So, the Schwinger transformation function for two infinitesimally closed points is written in terms of annihilation and creation processes of a particle as $F\left(x^{\prime} t^{\prime} ; x t\right)=\int d \underline{x}\left\langle x^{\prime} t^{\prime} \mid \underline{x} \underline{t}\right\rangle<\underline{x} \underline{t} \mid x t>$. It should be emphasized that since we do not understand the phenomenon of motion, then here it must suffice to expect that the state functions $\mid x, t>$ and $|\underline{x}, \underline{t}\rangle$ are quite different. Therefore, the intermediate motion state, $\mid \underline{x}, \underline{t}>$, can be defined on say motion space, $\underline{M}$, the points $\underline{x}(\underline{t})$ of which are all the motion atomic elements, $(\underline{x}(\underline{t}) \in \underline{M})$. To express Schwinger transformation function, $F$, as a path integral, we divide the finite time interval into $n+1$ intervals: $t=t_{0}, t_{1}, \ldots, t_{n+1}=t^{\prime} ; t_{k}=t_{0}+k \varepsilon$, where $\varepsilon$ can be made arbitrarily small by increasing $n$. In the limit $n \rightarrow \infty, F$ becomes an operational definition of the path integral. Hence, in general, in addition to background 4D Minkowski space $M_{4}$, also a background motion space $\underline{M}$, or say master space, MS $(\equiv \underline{M})$ is required. So, we now conceive of the two different spaces $M_{4}$ and MS, where the geometry of MS is a new physical entity, with degrees of freedom and a dynamics of its own. The above example imposes a constraint upon MS that it was embedded in $M_{4}$ as an indispensable individual companion to the particle, without relation to the other matter. In going into practical details, we further adopt the model discussed in previous section, which illustrates the problems of inertia effects, but it also hints at a possible solution. In accord, MS is not measurable directly, but it was argued that a deformation of MS is the origin of inertia effects that can be observed by us. We will not be concerned with the actual details of this model here, but only use it as a backdrop to study the motion of a particle. In general case of 3D motion in $M_{4}$, a flat MS is the 2D Minkowski space $\underline{M}_{2}$. In deriving the final step, we should compare and contrast the particle states of quantum fields defined on the background spaces $M_{4}$ and $\underline{M}_{2}$, forming a basis in the Hilbert space. It is quite clear that the following properties, being the essence of the chain of transformations for the finite time interval, hold:

1. There should be a particular way of going from each point $x_{i-1}\left(t_{i-1}\right) \in M_{4}$ to the intermediate motion point $\underline{x}_{i-1}\left(\underline{t}_{i-1}\right) \in \underline{M}_{2}$ and back $x_{i}\left(t_{i}\right) \in M_{4}$, such that the net result of each atomic double transformations is as if we had operated with a space-time translation on the original space $M_{4}$. So, the symmetry we are looking for must mix the particle quantum states during the motion in order to reproduce the central relationship between the two successive transformations of this symmetry and the generators of space-time translations. Namely, the subsequent operation of two finite transformations will induce a translation in space and time of the states on which they operate.

2. These successive transformations induce in $M_{4}$ the inhomogeneous Lorentz group, or Poincaré group, and that an unitary linear transformation $|x, t>\rightarrow U(\Lambda, a)| x, t>$ on vectors in the physical Hilbert space.

Thus, the underlying algebraic structure of this symmetry generators closes with the algebra of translations on the original space $M_{4}$ in a way that it can then be summarized as a non-trivial extension of the Poincaré group algebra, including the generators of translations. The only symmetry possessing such properties is the SUSY, which is accepted as a legitimate feature of nature, although it has never been experimentally observed. Certainly we now need to modify the standard theory to have MS-SUSY, involving a superspace which is an enlargement of a direct sum of background 
A new look at some aspects of geometry, particle physics, inertia, radiation and cosmology

spaces $M_{4} \oplus \underline{M}_{2}$ by the inclusion of additional fermion coordinates. Thereby an attempt will be made to treat the uniform motion of a particle as a complex process of the global (or rigid) MSSUSY double transformations (Ter-Kazarian, 2013b). Namely a particle undergoes to an infinite number of successive transitions from $M_{4}$ to $\underline{M}_{2}$ and back going permanently through fermion-boson transformations, which can be interpreted as its creation and annihilation processes occurring in $M_{4}$ or $\underline{M}_{2}$. We derive the Lorentz code of motion in terms of spinors referred to MS. This allows to introduce the physical finite time interval between two events, as integer number of the duration time of atomic double transition of a particle from $M_{4}$ and back. While all the particles are living on $M_{4}$, their superpartners can be viewed as living on $\underline{M}_{2}$.

\subsection{MS revisited}

According to previous section, we assume that a flat MS is the 2D Minkowski space: $\underline{M}_{2}=$ $R_{(+)}^{1} \oplus R_{(-)}^{1}$. The ingredient 1D-space $R_{\underline{m}}^{1}$ is spanned by the coordinates $\eta^{\underline{m}}$. The following notational conventions are used throughout this paper: all magnitudes related to the space $\underline{M}_{2}$ will be underlined. In particular, the underlined lower case Latin letters $\underline{m}, \underline{n}, \ldots=( \pm)$ denote the world indices related to $\underline{M}_{2}$. The metric in $\underline{M}_{2}$ is $\underline{g}=\underline{g}\left(\underline{e}_{m}, \underline{e}_{n}\right) \underline{\vartheta} \underline{\underline{m}} \otimes \underline{\underline{\vartheta}} \underline{\underline{n}}$, where $\underline{\vartheta} \underline{\underline{m}}=d \eta \underline{m}$ is the infinitesimal displacement. The basis $\underline{e}_{\underline{m}}$ at the point of interest in $\underline{M}_{2}$ is consisted of the two real null vectors: $\underline{g}\left(\underline{e}_{\underline{m}}, \underline{e_{n}}\right) \equiv<\underline{e}_{\underline{m}}, \underline{e}_{\underline{n}}>={ }^{*} o_{\underline{m} \underline{n}}, \quad\left({ }^{*} o_{\underline{m} \underline{n}}\right)=\left(\begin{array}{cc}0 & 1 \\ 1 & 0\end{array}\right)$. The norm, $i \bar{d} \equiv d \hat{\eta}$, given in the basis reads $i \underline{d}=\underline{e \vartheta}=\underline{e}_{\underline{m}} \otimes \underline{\vartheta} \underline{m}$, where $i \underline{d}$ is the tautological tensor field of type $(1,1)$, $\underline{e}$ is a shorthand for the collection of the 2-tuplet $\left(\underline{e}_{(+)}, \underline{e}_{(-)}\right)$, and $\underline{\vartheta}=\left(\underline{\underline{\vartheta}}^{(+)}\right)$. We may equivalently use a temporal $q^{0} \in T^{1}$ and a spatial $q^{1} \in R^{1}$ variables $q^{r}\left(q^{0}, q^{1}\right)(r=0,1)$, such that $\underline{M}_{2}=R^{1} \oplus T^{1}$. The norm, $i \underline{d}$, now can be rewritten in terms of displacement, $d q^{r}$, as $i \underline{d}=d \hat{q}=e_{0} \otimes d q^{0}+e_{1} \otimes d q^{1}$, where $e_{0}$ and $e_{1}$ are, respectively, the temporal and spatial basis vectors: $e_{0}=\frac{1}{\sqrt{2}}\left(\underline{e}_{(+)}+\underline{e}_{(-)}\right)$, $e_{1}=\frac{1}{\sqrt{2}}\left(\underline{e}_{(+)}-\underline{e}_{(-)}\right), \underline{g}\left(e_{r}, e_{s}\right) \equiv<e_{r}, e_{s}>=o_{r s},\left(o_{r s}\right)=\left(\begin{array}{cc}1 & 0 \\ 0 & -1\end{array}\right)$. The $\underline{M}_{2}$-companion is smoothly (injective and continuous) embedded in the $M_{4}$. Suppose the position of the particle in the background $M_{4}$ space is specified by the coordinates $x^{m}(s)(m=0,1,2,3)\left(x^{0}=t\right)$ with respect to the axes of the inertial system $S_{(4)}$. Then, a smooth map $f: \underline{M}_{2} \longrightarrow M_{4}$ is defined to be an immersion - an embedding which is a function that is a homeomorphism onto its image: $q^{0}=\frac{1}{\sqrt{2}}\left(\eta^{(+)}+\eta^{(-)}\right)=t$, $q^{1}=\frac{1}{\sqrt{2}}\left(\eta^{(+)}-\eta^{(-)}\right)=|\vec{x}|$. To motivate why is the MS two dimensional, we note that only two dimensional constructions of real null vectors are allowed as the basis at given point in MS, which can be embedded in the $(3+1)$-dimensional spacetime. This theory is mathematically somewhat similar to the more recent membrane theory, so the $\underline{M}_{2}$ can be viewed as $2 \mathrm{D}$ space living on the $4 \mathrm{D}$ world sheet. Given the inertial frame $S_{(4)}$ in $M_{4}$, we may define the corresponding inertial frame $S_{(2)}$ used by the non-accelerated observer for the position $q^{r}$ of a free particle in flat $\underline{M}_{2}$. Thereby the time axes of the two systems $S_{(2)}$ and $S_{4}$ coincide in direction and that the time coordinates are taken the same, $q^{0}=t$. For the case at hand, $v^{( \pm)}=\frac{d \eta^{( \pm)}}{d q^{0}}=\frac{1}{\sqrt{2}}\left(1 \pm v_{q}\right), v_{q}=\frac{d q^{1}}{d q^{0}}=|\vec{v}|=\left|\frac{d \vec{x}}{d t}\right|$. So the particle may be viewed as moving simultaneously in $M_{4}$ and $\underline{M}_{2}$. Hence, given the inertial frames $S_{(4)}, S_{(4)}^{\prime}$, $S_{(4)}^{\prime \prime}, \ldots$ in $M_{4}$, in this manner we may define the corresponding inertial frames $S_{(2)}, S_{(2)}^{\prime}, S_{(2)}^{\prime \prime}, \ldots$ in $\underline{M}_{2}$. Suppose the elements of the Hilbert space can be generated by the action of field-valued operators $\phi(x)(\chi(x), A(x)) \quad\left(x \in M_{4}\right)$, where $\chi(x)$ is the Weyl fermion and $A(x)$ is the complex scalar bosonic field defined on $M_{4}$, and accordingly, of field-valued operators $\phi(\eta)(\chi(\eta), \underline{A}(\eta)) \quad\left(\eta \in \underline{M}_{2}\right)$, where $\chi(\eta)$ is the Weyl fermion and $\underline{A}(\eta)$ is the complex scalar bosonic field defined on $\underline{M}_{2}$, on a translationally invariant vacuum: $|x>=\phi(x)| 0>, \quad\left|x_{1}, x_{2}>=\phi\left(x_{1}\right) \phi\left(x_{2}\right)\right| 0>\quad$ referring to $M_{4}$, $|\eta>=\phi(\eta)| 0>, \quad\left|\eta_{1}, \eta_{2}>=\phi\left(\eta_{1}\right) \phi\left(\eta_{2}\right)\right| 0>\quad$ referring to $\underline{M}_{2}$, etc. The displacement of the field takes the form $\phi\left(x_{1}+x_{2}\right)=e^{i x_{2}^{m} P_{m}} \phi\left(x_{1}\right) e^{-i x_{2}^{m} P_{m}}, \phi\left(\eta_{1}+\eta_{2}\right)=e^{i \eta_{2}^{\underline{m}} P_{\underline{m}}} \phi\left(\eta_{1}\right) e^{-i \eta_{2}^{\underline{m}} P_{\underline{m}}}$, where $P_{m}=i \partial_{m}$ is the generator of translations on quantum fields $\phi(x)$, and $\underline{P}_{\underline{m}}=i \underline{\partial}_{\underline{m}}$ is the generator of translations on quantum fields $\phi(\eta) \equiv \phi\left(t, q^{1}\right)$. According to the embedding map, the relation between the fields $\phi(x)$ and $\underline{\phi}(\eta)$ can be given by the a proper orthochronous Lorentz transformation. For a 

$\exp (-i \vec{\theta} \cdot \vec{S}-i \vec{\zeta} \cdot \vec{K})_{\alpha}{ }^{\beta} \phi_{\beta}(x)$, where $\vec{\theta}$ is the rotation angle about an axis $\vec{n}(\vec{\theta} \equiv \theta \vec{n})$, and $\vec{\zeta}$ is the boost vector $\vec{\zeta} \equiv \vec{e}_{v} \cdot \tan h^{-1} \beta$, provided $\vec{e}_{v} \equiv \vec{v} /|\vec{v}|, \beta \equiv|\vec{v}| / c, \theta^{i} \equiv(1 / 2) \varepsilon^{i j k} \theta_{k}(i, j, k=1,2,3)$, and $\zeta^{i} \equiv \theta^{i 0}=-\theta^{0 i}$. The antisymmetric tensor $S_{m n}=-S_{n m}$, satisfying the commutation relations of the $S L(2 . C)$, is the (finite-dimensional) irreducible matrix representations of the Lie algebra of the Lorentz group, and $\alpha$ and $\beta$ label the components of the matrix representation space, the dimension of which is related to the spin $S^{i} \equiv(1 / 2) \varepsilon^{i j k} S_{k}$ of the particle. The spin $\vec{S}$ generates three-dimensional rotations in space and the $K^{i} \equiv S^{0 i}$ generate the Lorentz-boosts. The fields of spin-zero $(\vec{S}=\vec{K}=0)$ scalar field $A(x)$ and spin-one $A^{n}(x)$, corresponding to the (1/2.1/2) representation, transform under a general Lorentz transformation as $\underline{A}(\eta)=A(x)$, spin $0 ; \quad \underline{A}^{m}(\eta)=\Lambda_{n}^{m} A^{n}(x)$, spin 1 , where the Lorentz transformation is written as $\Lambda_{n}^{m}(M) \equiv \frac{1}{2} \operatorname{Tr}\left(\sigma_{m} M \sigma_{n} M^{\dagger}\right)$, provided, $\sigma^{m} \equiv\left(I_{2}, \vec{\sigma}\right), \vec{\sigma}$ are Pauli spin matrices. A two-component $(1 / 2,0)$ Weyl fermion $\chi_{\beta}(x)$ transforms under Lorentz transformation, in accord to embedding map, as $\chi_{\beta}(x) \longrightarrow \underline{\chi}_{\alpha}(\eta)=\left(M_{R}\right)_{\alpha}{ }^{\beta} \chi_{\beta}(x), \alpha, \beta=1,2$ where the rotation matrix is given as $M_{R}=e^{i \frac{1}{2} \sigma_{2} \theta_{2}} e^{i \frac{1}{2} \sigma_{3} \theta_{3}}$. The matrix $M_{R}$ corresponds to the rotation of an hermitian $2 \times 2$ matrix $p^{n} \sigma_{n}: p_{q}^{m} \sigma_{m}=M_{R} p^{n} \sigma_{n} M_{R}^{\dagger}$, by the angles $\theta_{3}$ and $\theta_{2}$ about the axes $n_{3}$ and $n_{2}$, respectively, where the standard momentum is $p^{n} \equiv m\left(\operatorname{ch} \beta, \operatorname{sh} \beta \sin \theta_{2} \cos \theta_{3}, \operatorname{sh} \beta \sin \theta_{2} \sin \theta_{3}, \operatorname{sh} \beta \cos \theta_{2}\right)$, and $p_{q}^{m}$ is $p_{q}^{m} \equiv m(\operatorname{ch} \beta, 0,0, \operatorname{sh} \beta)$. The two-component $(0,1 / 2)$ Weyl spinor field is denoted by $\bar{\chi}^{\dot{\beta}}(x)$, and transforms as $\bar{\chi}^{\dot{\beta}}(x) \longrightarrow \underline{\bar{\chi}}^{\dot{\alpha}}(\eta)=\left(M_{R}^{-1}\right)_{\dot{\beta}}^{\dagger \dot{\alpha}} \bar{\chi}^{\dot{\beta}}(x), \dot{\alpha}, \dot{\beta}=1,2$ where we have used $\left(M^{\dagger}\right)_{\dot{\alpha}}^{\dot{\beta}}=\left(M^{*}\right)_{\dot{\alpha}}^{\dot{\beta}}$. The so-called 'dotted' indices have been introduced to distinguish the $(0,1 / 2)$ representation from the $(1 / 2,0)$ representation. The "bar" over the spinor is a convention that this is the $(0,1 / 2)$ representation. The infinitesimal Lorentz transformation matrices for the $(1 / 2,0)$ and $(0,1 / 2)$ representations, $M \simeq I_{2}-\frac{i}{2} \vec{\theta} \cdot \vec{\sigma}-\frac{1}{2} \vec{\zeta} \cdot \vec{\sigma}, \quad$ for $\left(\frac{1}{2}, 0\right) ; \quad\left(M^{-1}\right)^{\dagger} \simeq I_{2}-\frac{i}{2} \vec{\theta} \cdot \vec{\sigma}+\frac{1}{2} \vec{\zeta} \cdot \vec{\sigma}, \quad$ for $\quad\left(0, \frac{1}{2}\right)$ give $S^{m n}=$ $\sigma^{m n}$ for the $(1 / 2,0)$ representation and $S^{m n}=\bar{\sigma}^{m n}$ for the $(0,1 / 2)$ representation, where the bilinear covariants that transform as a Lorentz second-rank tensor read $\left(\sigma^{m n}\right)_{\alpha}{ }^{\beta} \equiv \frac{i}{4}\left(\sigma_{\alpha \dot{\alpha}}^{m} \bar{\sigma}^{n \dot{\alpha} \beta}-\sigma_{\alpha \dot{\alpha}}^{n} \bar{\sigma}^{m \dot{\alpha} \beta}\right)$, $\left(\bar{\sigma}^{m n}\right)_{\dot{\beta}}^{\dot{\alpha}} \equiv \frac{i}{4}\left(\bar{\sigma}^{m \dot{\alpha} \alpha} \sigma_{\alpha \dot{\beta}}^{n}-\bar{\sigma}^{n \dot{\alpha} \alpha} \sigma_{\alpha \dot{\beta}}^{m}\right)$, provided $\bar{\sigma}^{m} \equiv\left(I_{2} ;-\vec{\sigma}\right),\left(\sigma^{m *}\right)_{\alpha \dot{\beta}}=\sigma_{\beta \dot{\alpha}}^{m}$ and $\left(\bar{\sigma}^{m *}\right)^{\dot{\alpha} \beta}=\bar{\sigma}^{m \dot{\beta} \alpha}$.

\subsection{MS-SUSY}

As alluded to above (Ter-Kazarian, 2013b), a creation of a particle in $\underline{M}_{2}$ means its transition from $M_{4}$ to $\underline{M}_{2}$, while an annihilation of a particle in $\underline{M}_{2}$ means vice versa. The same interpretation holds for the creation and annihilation processes in $M_{4}$. Since all fermionic and bosonic states, taken together, form a basis in the Hilbert space, the basis vectors in the Hilbert space, therefore, can be written in the form $\mid \underline{n}_{b}, n_{f}>$ or $\mid n_{b}, \underline{n}_{f}>$, where the boson and fermion occupation numbers are $n_{b}$ or $\underline{n}_{b}(=0,1, \ldots, \infty)$ and $n_{f}$ or $\underline{n}_{f}(=0,1)$. So, we may construct the quantum operators, $\left(q^{\dagger}, \underline{q}^{\dagger}\right)$ and $(q, \underline{q})$, which replace bosons by fermions and fermions by bosons, respectively, $q^{\dagger} \mid \underline{n}_{b}, n_{f}>\longrightarrow$ $\left|\underline{n}_{b}-1, n_{f}+1>, q\right| \underline{n}_{b}, n_{f}>\longrightarrow \mid \underline{n}_{b}+1, n_{f}-1>$, and that $\underline{q}^{\dagger}\left|n_{b}, \underline{n}_{f}>\longrightarrow\right| n_{b}-1, \underline{n}_{f}+1>$, $\underline{q}\left|n_{b}, \underline{n}_{f}>\longrightarrow\right| n_{b}+1, \underline{n}_{f}-1>$. This framework combines bosonic and fermionic states on the same footing, rotating them into each other under the action of operators $q$ and $q$. Consider two pairs of creation and annihilation operators $\left(b^{\dagger}, b\right)$ and $\left(f^{\dagger}, f\right)$ for bosons and fermions, respectively, referred to the background space $M_{4}$, as well as $\left(\underline{b}^{\dagger}, \underline{b}\right)$ and $\left(\underline{f}^{\dagger}, \underline{f}\right)$ for bosons and fermions, respectively, related to the background master space $\underline{M}_{2}$. Putting two operators in one $B=(\underline{b}$ or $b)$ and $F=(f$ or $f$ ), the canonical quantization rules can be written most elegantly as $\left[B, B^{\dagger}\right]=1 ; \quad\left\{F, F^{\dagger}\right\}=1$; $[B, B]=\left[B^{\dagger}, B^{\dagger}\right]=\{F, F\}=\left\{F^{\dagger}, F^{\dagger}\right\}=[B, F]=\left[B, F^{\dagger}\right]=\left[B^{\dagger}, F\right]=\left[B^{\dagger}, F^{\dagger}\right]=0$, where we note that $\delta_{i j} \delta^{3}\left(\vec{p}-\vec{p}^{\prime}\right)$ and $\delta_{i j} \delta^{3}\left(\vec{p}_{q}-\vec{p}_{q}^{\prime}\right)$ are the unit element 1 of the convolution product $*$, and according to embedding map we have $p_{q}= \pm|\vec{p}|$ and $p_{q}^{\prime}= \pm\left|\vec{p}^{\prime}\right|$. The operators $q$ and $\underline{q}$ can be constructed as $q^{\dagger}=q_{0} \underline{b} f^{\dagger}, q=q_{0}, \underline{b}^{\dagger} f, \quad q^{\dagger}=q_{0} b f^{\dagger}, \quad q=q_{0} b^{\dagger} f$. So, we may refer the action of the supercharge operators $q$ and $q^{\dagger}$ to the background space $M_{4}$, having applied in the chain of following transformations of fermion $\chi$ (accompanied with the auxiliary field $F$ as it will be seen later on) to boson $\underline{A}$, defined on $\underline{M}_{2}: \cdots \longrightarrow \chi^{(F)} \longrightarrow \underline{A} \longrightarrow \chi^{(F)} \longrightarrow \underline{A} \longrightarrow \chi^{(F)} \longrightarrow \cdots$ Respectively, we may refer the action of the supercharge operators $\underline{q}$ and $\underline{q}^{\dagger}$ to the $\underline{M}_{2}$, having applied in the chain of following transformations of fermion $\underline{\chi}$ (accompanied with the auxiliary field $\underline{F}$ ) to boson $A$, defined 
on the background space $M_{4}: \cdots \longrightarrow \underline{\chi}^{(\underline{F})} \longrightarrow A \longrightarrow \underline{\chi}^{(\underline{F})} \longrightarrow A \longrightarrow \underline{\chi}^{(\underline{F})} \longrightarrow \cdots$ Written in one notation, $Q=(q$ or $\underline{q})$, the operators become $Q=q_{0} B^{\dagger} F=(q$ or $\underline{q}), Q^{\dagger}=q_{0} B F^{\dagger}=\left(q^{\dagger}\right.$ or $\left.\underline{q}^{\dagger}\right)$. Due to nilpotent fermionic operators $F^{2}=\left(F^{\dagger}\right)^{2}=0$, the operators $Q$ and $Q \dagger$ also are nilpotent: $Q^{2}=\left(Q^{\dagger}\right)^{2}=0$. Hence, the quantum system can be described in one notation by the selfadjoint Hamiltonian $\mathcal{H}=\left(H_{q} \equiv\left\{q^{\dagger}, q\right\}\right.$ or $\left.H_{q} \equiv\left\{\underline{q}^{\dagger}, \underline{q}\right\}\right)$, and that the generators $Q$ and $Q^{\dagger}$ satisfy an algebra of anticommutation and commutation relations: $\mathcal{H}=\left\{Q^{\dagger}, Q\right\} \geq 0 ; \quad[\mathcal{H}, Q]=\left[\mathcal{H}, Q^{\dagger}\right]=0$. This is a sum of Hamiltonian of bosonic and fermionic noninteracting oscillators, which decouples, for $Q=q$, into $H_{q}=q_{0}^{2}\left(\underline{b}^{\dagger} \underline{b}+f^{\dagger} f\right)=q_{0}^{2}\left(\underline{b}^{\dagger} \underline{b}+\frac{1}{2}\right)+q_{0}^{2}\left(f^{\dagger} f-\frac{1}{2}\right) \equiv H_{\underline{b}}+H_{f}$, or, for $Q=\underline{q}$, into $H_{q}=q_{0}^{2}\left(b^{\dagger} b+\underline{f}^{\dagger} f\right)=q_{0}^{2}\left(b^{\dagger} b+\frac{1}{2}\right)+q_{0}^{2}\left(\underline{f}^{\dagger} f-\frac{1}{2}\right) \equiv H_{b}+H_{f}$, with the corresponding energies: $E_{q}=q_{0}^{2}\left(\underline{n}_{b}+\frac{1}{2}\right)+q_{0}^{2}\left(n_{f}-\frac{1}{2}\right), E_{q}=q_{0}^{2}\left(n_{b}+\frac{1}{2}\right)+q_{0}^{2}\left(\underline{n}_{f}-\frac{1}{2}\right)$. This formalism manifests its practical and technical virtue in the proposed algebra, which becomes more clear in a normalization $q_{0}=\sqrt{\mathrm{m}}$ : $\left\{Q^{\dagger}, Q\right\}=2 m ; \quad\{Q, Q\}=\left\{Q^{\dagger}, Q^{\dagger}\right\}=0$. The latter has underlying algebraic structure of the superalgebra for massive one-particle states in the rest frame of $N=1$ SUSY theory without central charges. This is rather technical topic, and it requires care to do correctly. In what follows we only give a brief sketch. The extension of the MS-SUSY superalgebra in general case when $\vec{p}=i \vec{\partial} \neq 0$ in $M_{4}$ or $p_{q}=i \partial_{q} \neq 0$ in $\underline{M}_{2}$, and assuming that the resulting motion of a particle in $M_{4}$ is governed by the Lorentz symmetries, the MS-SUSY algebra can then be summarized as a non-trivial extension of the Poincaré group algebra thus of the commutation relations of the bosonic generators of four momenta and six Lorentz generators referred to $M_{4}$. Moreover, if there are several spinor generators $Q_{\alpha}{ }^{i}$ with $i=1, \ldots, N$ - theory with $N$-extended supersymmetry, can be written as a graded Lie algebra (GLA) of SUSY field theories, with commuting and anticommuting generators: $\left\{Q_{\alpha}{ }^{i}, \bar{Q}^{j}{ }_{\dot{\alpha}}\right\}=2 \delta^{i j} \sigma_{\alpha \dot{\alpha}}^{\hat{m}} p_{\hat{m}}$; $\left[p_{\hat{m}}, Q_{\alpha}{ }^{i}\right]=\left[p_{\hat{m}}, \bar{Q}^{j}{ }_{\dot{\alpha}}\right]=0,\left\{Q_{\alpha}{ }^{i}, Q_{\beta}{ }^{j}\right\}=\left\{\bar{Q}^{i}{ }_{\alpha}, \bar{Q}_{\dot{\beta}}^{j}\right\}=0 ;\left[p_{\hat{m}}, p_{\hat{n}}\right]=0$. Here $\sigma^{( \pm)}=(1 / 2)\left(\sigma^{o} \pm \sigma^{3}\right)$, and in order to trace a maximal resemblance in outward appearance to the standard SUSY theories, we set one notation $\hat{m}=(m$ if $Q=q$, or $\underline{m}$ if $Q=q)$, no sum over $\hat{m}$, and as before the indices $\alpha$ and $\dot{\alpha}$ go over 1 and 2 . So for both supercharges, $q$ and $q$, we get a supersymmetric models, respectively: $\left\{q_{\alpha}^{i}, \bar{q}_{\dot{\alpha}}^{j}\right\}=2 \delta^{i j} \sigma_{\alpha \dot{\alpha}}^{m} p_{m} ;\left[p_{m}, q_{\alpha}^{i}\right]=\left[p_{m}, \bar{q}_{\dot{\alpha}}^{j}\right]=0,\left\{q_{\alpha}^{i}, q_{\beta}^{j}\right\}=\left\{\bar{q}_{\dot{\alpha}}^{i}, \bar{q}_{\dot{\beta}}^{j}\right\}=0 ; \quad\left[p_{m}, p_{n}\right]=0$. and $\left\{\underline{q}_{\alpha}^{i}, \underline{\bar{q}}_{\dot{\alpha}}^{j}\right\}=2 \delta^{i j} \sigma_{\alpha \dot{\alpha}}^{\underline{m}} p_{\underline{m}} ;\left[p_{\underline{m}}, \underline{q}_{\alpha}^{i}\right]=\left[p_{\underline{m}}, \underline{\bar{q}}_{\dot{\alpha}}^{j}\right]=0,\left\{\underline{q}_{\alpha}^{i}, \underline{q}_{\beta}^{j}\right\}=\left\{\underline{\bar{q}}_{\dot{\alpha}}^{i}, \underline{\bar{q}}_{\dot{\beta}}^{j}\right\}=0 ;\left[p_{\underline{m}}, p_{\underline{n}}\right]=0$. For the self-contained arguments, we should emphasize the crucial differences between the MS-induced SUSY and the standard theories as follows:

1) The standard theory can be realized only as a spontaneously broken symmetry since the experiments do not show elementary particles to be accompanied by superpartners with different spin but identical mass. The MS-SUSY, in contrary, can only be realized as an unbroken SUSY.

2) In the standard theory, the Q's operate on the fields defined on the single $M_{4}$ space. It is why the result of a Lorentz transformation in $M_{4}$ followed by a supersymmetry transformation is different from that when the order of the transformations is reversed. But, in the MS-SUSY theory, the Q-operators operate on the fields defined on both $M_{4}$ and $\underline{M}_{2}$ spaces, fulfilling a transition of a particle between these spaces $\left(M_{4} \rightleftharpoons \underline{M}_{2}\right)$. So after a Lorentz transformation in $M_{4}$ followed by a supersymmetry transformation (which, as we shall see below, now results to uniform motion of a particle with initial constant velocity) we have a particle moving with changed constant velocity. We obtain the same result if we reverse the order of the transformations, namely a Lorentz transformation changes the initial velocity and a supersymmetry transformation followed by a Lorentz transformation just keep the uniform motion with the changed velocity.

We shall forbear to write out further the unitary representations of supersymmetry, giving rise to the notion of supermultiplets, as they are so well known. Also, unless otherwise stated we will not discuss the theories with $N>1$, because it is unlikely that they play any role in low-energy physics.

\subsection{Wess-Zumino model}

To obtain a feeling for this model we may consider first example of non-trivial linear representation of the MS-SUSY algebra in analogy of the Wess-Zumino toy model, which has $N=1$ and $s_{0}=0$, and contains two spin states of a massive Majorana spinor $\psi(\chi, \underline{\chi})$ and two complex scalar fields $\mathcal{A}(A, \underline{A})$ and auxiliary fields $\mathcal{F}(F, \underline{F})$, which provide in supersymmetry theory the 
fermionic and bosonic degrees of freedom to be equal. This model is instructive because it contains the essential elements of the MS-induced SUSY. Let us first introduce four additional, anticommuting (Grassmann) parameters $\epsilon^{\alpha}\left(\xi^{\alpha}, \underline{\xi}^{\alpha}\right)$ and $\bar{\epsilon}^{\alpha}\left(\bar{\xi}^{\alpha}, \bar{\xi}^{\alpha}\right):\left\{\epsilon^{\alpha}, \epsilon^{\beta}\right\}=\left\{\bar{\epsilon}^{\alpha}, \bar{\epsilon}^{\beta}\right\}=\left\{\epsilon^{\alpha}, \bar{\epsilon}^{\beta}\right\}=0$, $\left\{\epsilon^{\alpha}, Q_{\beta}\right\}=\cdots=\left[p_{\hat{m}}, \epsilon^{\alpha}\right]=0$, which allow to write the algebra (??) $(N=1)$ in terms of commutators only: $[\epsilon Q, \bar{Q} \bar{\epsilon}]=2 \epsilon \sigma^{\hat{m}} \bar{\epsilon} p_{\hat{m}},[\epsilon Q, \epsilon Q]=[\bar{Q} \bar{\epsilon}, \bar{Q} \bar{\epsilon}]=\left[p^{\hat{m}}, \epsilon Q\right]=\left[p^{\hat{m}}, \bar{Q} \bar{\epsilon}\right]=0$. Here we have dropped the indices $\epsilon Q=\epsilon^{\alpha} Q_{\alpha}$ and $\bar{\epsilon} \bar{Q}=\bar{\epsilon}_{\dot{\alpha}} \bar{Q}^{\dot{\alpha}}$. The infinitesimal supersymmetry transformations for $Q=q$ read $\delta_{\xi} \underline{A}=(\xi q+\bar{\xi} \bar{q}) \times \underline{A}=\sqrt{2} \xi \chi, \delta_{\xi} \chi=(\xi q+\bar{\xi} \bar{q}) \times \chi=i \sqrt{2} \sigma^{m} \bar{\xi} \partial_{m} \underline{A}+\sqrt{2} \xi F$, $\delta_{\xi} F=(\xi q+\bar{\xi} \bar{q}) \times F=i \sqrt{2} \bar{\xi} \bar{\sigma}^{m} \partial_{m} \chi$; and for $Q=\underline{q}$ are in the form $\delta_{\underline{\xi}} A=(\underline{\xi} \underline{q}+\underline{\bar{\xi}} \bar{q}) \times A=\sqrt{2} \underline{\xi} \underline{\chi}$, $\delta_{\underline{\xi}} \underline{\chi}=(\underline{\xi} \underline{q}+\underline{\bar{\xi}} \underline{q}) \times \underline{\chi}=i \sqrt{2} \sigma \underline{m} \underline{\bar{\xi}} \partial_{\underline{m}} A+\sqrt{2} \underline{\xi} \underline{F}, \delta_{\underline{\xi}} \underline{F}=(\underline{\xi} \underline{q}+\underline{\bar{\xi}} \underline{\bar{q}}) \times \underline{F}=i \sqrt{2} \underline{\bar{\xi}} \overline{\bar{\sigma}} \underline{\underline{m}} \partial_{\underline{m}} \underline{\chi}$, where $A=\underline{A}$. The first relation means that there should be a particular way of going from one subspace (bosonic/fermionic) to the other and back, such that the net result is as if we had operator of translation $p_{\hat{m}}$ on the original subspace. Actually, it can be checked that the supersymmetry transformations close supersymmetry algebra: $\left[\delta_{\epsilon_{1}}, \delta_{\epsilon_{2}}\right] \mathcal{A}=-2 i\left(\epsilon_{1} \sigma^{\hat{m}} \bar{\epsilon}_{2}-\epsilon_{2} \sigma^{\hat{m}} \bar{\epsilon}_{1}\right) \partial_{\hat{m}} \mathcal{A}$, and likewise for $\psi$ and $\mathcal{F}$. In the framework of MS-SUSY theory, the Wess-Zumino model has the following Lagrangians: $\mathcal{L}_{Q=q}=\mathcal{L}_{0}+$ $m \mathcal{L}_{m}, \quad \mathcal{L}_{Q=q}=\underline{\mathcal{L}}_{0}+m \underline{\mathcal{L}}_{m}$, provided, $\mathcal{L}_{0}=i \partial_{m} \bar{\chi} \bar{\sigma}^{m} \chi+\underline{A}^{*} \square \underline{A}+F^{*} F, \mathcal{L}_{m}=\underline{A} F+\underline{A}^{*} F^{*}-\frac{1}{2} \chi \chi-\frac{1}{2} \bar{\chi} \bar{\chi}$, $\underline{\mathcal{L}}_{0}=i \partial_{\underline{m}} \underline{\bar{\chi}}^{\bar{\sigma}}{ }^{m} \underline{\chi}+A^{*} \square A+\underline{F}^{*} \underline{F}, \underline{\mathcal{L}}_{\underline{m}}=A \underline{F}+A^{*} \underline{F}^{*}-\frac{1}{2} \underline{\chi} \underline{\chi}-\frac{1}{2} \underline{\bar{\chi}} \underline{\bar{\chi}}$, where according to the embedding map, $\square=\square$ and $A=\underline{A}$. Whereupon, the equations of motion for the Weyl spinor $\psi$ and complex scalar $\mathcal{A}$ of the same mass $m$, are $(a)\left[i \bar{\sigma}^{m} \partial_{m} \chi+m \bar{\chi}=0, \quad i \bar{\sigma} \underline{\underline{\chi}} \partial_{\underline{m}} \underline{\chi}+m \underline{\bar{\chi}}=0, \quad F+m \underline{A}^{*}=0\right]$, or (b) $\left[\underline{F}+m A^{*}=0, \quad \square \underline{A}+m F^{*}=0, \quad \square A+m \underline{F}^{*}=0\right]$. Respectively, (a) stands for $Q=q$ (referring to the motion of a fermion, $\chi$, in $M_{4}$ ) and (b) stands for $Q=\underline{q}$ (so, of a boson, $A$, in $M_{4}$ ). Finally, the algebraic auxiliary field $\mathcal{F}$ can be eliminated to find $\mathcal{L}_{Q=q}=i \bar{\partial}_{m} \bar{\chi} \bar{\sigma}^{m} \chi-\frac{1}{2}(\chi \chi+\bar{\chi} \bar{\chi})+\underline{A^{*}} \underline{\square} \underline{A}-m^{2} \underline{A}^{*} \underline{A}$, $\underline{\mathcal{L}}_{Q=\underline{q}}=i \partial_{\underline{m}} \underline{\bar{\chi}}^{m} \underline{\underline{\chi}}-\frac{1}{2}(\underline{\chi} \underline{\chi}+\underline{\bar{\chi}} \underline{\bar{\chi}})+A^{*} \square A-m^{2} A^{*} A$.

\subsection{General superfields}

In the framework of standard generalization of the coset construction, we will take $G=G_{q} \times G_{q}$ to be the supergroup generated by the MS-SUSY algebra (??). Let the stability group $H=H_{q} \times H_{q}$ be the Lorentz group (as to $M_{4}$ and $\underline{M}_{2}$ ), and we choose to keep all of $G$ unbroken. Given $G$ and $H$, we can construct the coset, $G / H$, by an equivalence relation on the elements of $G: \quad \Omega \sim \Omega h$, where $\Omega=\Omega_{q} \times \Omega_{\underline{q}} \in G$ and $h=h_{q} \times h_{q} \in H$, so that the coset can be pictured as a section of a fiber bundle with total space, $G$, and fiber, $H$. So, the Maurer-Cartan form, $\Omega^{-1} d \Omega$, is valued in the Lie algebra of $G$, and transforms as follows under a rigid $\mathrm{G}$ transformation, $\Omega \longrightarrow g \Omega h^{-1}, \quad \Omega^{-1} d \Omega \longrightarrow$ $h\left(\Omega^{-1} d \Omega\right) h^{-1}-d h h^{-1}$, with $g \in G$. Also we consider a superspace which is an enlargement of $M_{4} \oplus \underline{M}_{2}$ (spanned by the coordinates $X^{\hat{m}}=\left(x^{m}, \eta \underline{m}\right)$ by the inclusion of additional fermion coordinates $\Theta^{\alpha}=\left(\theta^{\alpha}, \underline{\theta}^{\alpha}\right)$ and $\bar{\Theta}_{\dot{\alpha}}=\left(\bar{\theta}_{\dot{\alpha}}, \bar{\theta}_{\dot{\alpha}}\right)$, as to $(q, q)$, respectively. But note that the relation between the two spinors $\theta$ and $\underline{\theta}$ should be derived further from the embedding map (see next subsection). These spinors satisfy the following relations: $\left\{\Theta^{\alpha}, \Theta^{\beta}\right\}=\left\{\bar{\Theta}_{\dot{\alpha}}, \bar{\Theta}_{\dot{\beta}}\right\}=\left\{\Theta^{\alpha}, \bar{\Theta}_{\dot{\beta}}\right\}=0$, $\left[x^{m}, \theta^{\alpha}\right]=\left[x^{m}, \bar{\theta}_{\dot{\alpha}}\right]=0,\left[\eta^{\underline{m}}, \underline{\theta}^{\alpha}\right]=\left[\eta \underline{m}, \underline{\theta}_{\dot{\alpha}}\right]=0$. and $\Theta^{\alpha *}=\bar{\Theta}^{\dot{\alpha}}$. Points in superspace are then identified by the generalized coordinates $z^{M}=\left(X^{\hat{m}}, \Theta^{\alpha}, \bar{\Theta}_{\dot{\alpha}}\right)$. In case at hand we have then $\Omega(X, \Theta, \bar{\Theta})=e^{i\left(-X^{\hat{m}} p_{\hat{m}}+\Theta^{\alpha} Q_{\alpha}+\bar{\Theta}_{\dot{\alpha}} \bar{Q}^{\dot{\alpha}}\right)}=\Omega_{q}(x, \theta, \bar{\theta}) \times \Omega_{q}(\eta, \underline{\theta}, \underline{\bar{\theta}})$, where we now imply a summation over $\hat{m}$, etc., such that $\Omega_{q}(x, \theta, \bar{\theta})=e^{i\left(-x^{m} p_{m}+\theta^{\alpha} q_{\alpha}+\bar{\theta}_{\dot{\alpha}} \bar{q}^{\dot{\alpha}}\right)}, \Omega_{\underline{q}}(\eta, \underline{\theta}, \underline{\bar{\theta}})=e^{i\left(-\eta \underline{m}^{\underline{m}} p_{\underline{m}}+\underline{\theta}^{\alpha} \underline{q}_{\alpha}+\underline{\bar{\theta}}_{\dot{\alpha}} \bar{q}^{\dot{\alpha}}\right)}$. Supersymmetry transformation will be defined as a translation in superspace, specified by the group element $g(0, \epsilon, \bar{\epsilon})=e^{i\left(\epsilon^{\alpha} Q_{\alpha}+\bar{\epsilon}_{\dot{\alpha}} \bar{Q}^{\dot{\alpha}}\right)}=g_{q}(0, \xi, \bar{\xi}) \times g_{q}(0, \underline{\xi}, \bar{\xi})=e^{i\left(\xi^{\alpha} q_{\alpha}+\bar{\xi}_{\dot{\alpha}} \bar{q}^{\dot{\alpha}}\right)} \times e^{i\left(\underline{\xi}^{\alpha} \underline{q}_{\alpha}+\underline{\xi}_{\dot{\alpha}} \bar{q}^{\dot{\alpha}}\right)}$, with corresponding anticommuting parameters $\epsilon=(\xi$ or $\bar{\xi})$. To study the effect of supersymmetry transformations and $h=1$, we consider $g(0, \epsilon, \bar{\epsilon}) \Omega(X, \Theta, \bar{\Theta})=e^{i\left(\epsilon^{\alpha} Q_{\alpha}+\bar{\epsilon}_{\dot{\alpha}} \bar{Q}^{\dot{\alpha}}\right)} e^{i\left(-X^{\hat{m}} p_{\hat{m}}+\Theta^{\alpha} Q_{\alpha}+\bar{\Theta}_{\dot{\alpha}} \bar{Q}^{\dot{\alpha}}\right)}$. The multiplication of two successive transformations can be computed with the help of the Baker-CampbellHausdorf formula $e^{A} e^{B}=e^{A+B+(1 / 2)[A, B]+\cdots}$. Hence the transformation above induces the motion $g(0, \epsilon, \bar{\epsilon}) \Omega\left(X^{\hat{m}}, \Theta, \bar{\Theta}\right) \longrightarrow\left(X^{\hat{m}}+i \Theta \sigma^{\hat{m}} \bar{\epsilon}-i \epsilon \sigma^{\hat{m}} \bar{\Theta}, \Theta+\epsilon, \bar{\Theta}+\bar{\epsilon}\right)$, namely, $g_{q}(0, \xi, \bar{\xi}) \Omega_{q}(x, \theta, \bar{\theta}) \longrightarrow$ $\left(x^{m}+i \theta \sigma^{m} \bar{\xi}-i \xi \sigma^{m} \bar{\theta}, \theta+\xi, \bar{\theta}+\bar{\xi}\right), g_{\underline{q}}(0, \underline{\xi}, \underline{\bar{\xi}}) \Omega_{\underline{q}}(\eta, \underline{\theta}, \underline{\bar{\theta}}) \longrightarrow\left(\eta^{\underline{m}}+i \underline{\theta} \sigma^{\underline{m}} \underline{\bar{\xi}}-i \underline{\xi} \sigma^{\underline{m}} \underline{\hat{\theta}}, \underline{\theta}+\underline{\xi}, \underline{\hat{\theta}}+\underline{\bar{\xi}}\right)$. The superfield $\Phi\left(z^{M}\right)$, which has a finite number of terms in its expansion in terms of $\Theta$ and $\bar{\Theta}$ owing to their anticommuting property, can be considered as the generator of the various components of the supermultiplets. We will consider only a scalar superfield $\Phi^{\prime}\left(z^{M^{\prime}}\right)=\Phi\left(z^{M}\right)$, an infinitesimal super- 
A new look at some aspects of geometry, particle physics, inertia, radiation and cosmology

symmetry transformation of which is given as $\delta_{\epsilon} \Phi\left(z^{M}\right)=\left(\epsilon^{\alpha} Q_{\alpha}+\bar{\epsilon}_{\dot{\alpha}} \bar{Q}^{\dot{\alpha}}\right) \times \Phi\left(z^{M}\right)$. Acting on this space of functions, the $Q$ and $\bar{Q}$ can be represented as differential operators: $Q_{\alpha}=\frac{\partial}{\partial \Theta^{\alpha}}-i \sigma_{\alpha \dot{\alpha}}^{\hat{m}} \bar{\Theta}^{\dot{\alpha}} \partial_{\hat{m}}$, $\bar{Q}^{\dot{\alpha}}=\frac{\partial}{\partial \Theta_{\dot{\alpha}}}-i \Theta^{\alpha} \sigma_{\alpha \dot{\beta}^{\hat{m}}}^{\dot{\beta} \dot{\alpha}} \partial_{\hat{m}}$, where, as usual, the undotted/dotted spinor indices can be raised and lowered with a two dimensional undotted/dotted $\varepsilon$-tensors, and the anticommuting derivatives obey the relations $\frac{\partial}{\partial \Theta^{\alpha}} \Theta^{\beta}=\delta_{\alpha}^{\beta}, \frac{\partial}{\partial \Theta^{\alpha}} \Theta^{\beta} \Theta^{\gamma}=\delta_{\alpha}^{\beta} \Theta^{\gamma}-\delta_{\alpha}^{\gamma} \Theta^{\beta}$, and similarly for $\bar{\Theta}$. In order to write the exterior product in terms of differential operators, one induces a new basis as $e^{A}(z)=d Z^{M} e_{M}^{A}(z)$, and that $D_{A}=e_{A}^{N}(z) \frac{\partial}{\partial z^{N}}$, where to be brief we left implicit the symbol $\wedge$ in writing of exterior product. The covariant derivative operators $D_{\hat{m}}=\partial_{\hat{m}}, D_{\alpha}=\frac{\partial}{\partial \Theta^{\alpha}}+i \sigma_{\alpha \dot{\alpha}}^{\hat{m}} \bar{\Theta}^{\dot{\alpha}} \partial_{\hat{m}}, \bar{D}^{\dot{\alpha}}=\frac{\partial}{\partial \Theta_{\dot{\alpha}}}+i \Theta^{\alpha} \sigma^{\hat{m}}{ }_{\alpha \dot{\beta}} \varepsilon^{\dot{\beta} \dot{\alpha}} \partial_{\hat{m}}$, anticommute with the $Q$ and $\bar{Q}\left\{Q_{\alpha}, D_{\beta}\right\}=\left\{\bar{Q}_{\dot{\alpha}}, \bar{D}_{\dot{\beta}}\right\}=\left\{Q_{\alpha}, \bar{D}_{\dot{\beta}}\right\}=\left\{\bar{Q}_{\dot{\alpha}}, D_{\beta}\right\}=0$, and satisfy the following structure relations: $\left\{D_{\alpha}, D_{\dot{\alpha}}\right\}=-2 i \sigma_{\alpha \dot{\alpha}}^{\hat{m}} \partial_{\hat{m}},\left\{D_{\alpha}, D_{\beta}\right\}=\left\{\bar{D}_{\dot{\alpha}}, \bar{D}_{\dot{\beta}}\right\}=0$. Then we obtain $e_{A}{ }_{A}^{M}=\left(\begin{array}{ccc}e_{\hat{a}}^{\hat{m}}=\delta_{\hat{a}}^{\hat{m}} & e_{\hat{a}}{ }^{\mu}=0 & e_{\hat{a} \dot{\mu}}=0 \\ e_{\alpha}^{\hat{m}}=i \sigma_{\alpha \dot{\alpha}}^{\hat{m}} \bar{\Theta}^{\dot{\alpha}} & e_{\alpha}^{\mu}=\delta_{\alpha}^{\mu} & e_{\alpha \dot{\mu}}=0 \\ e^{\dot{\alpha} \hat{m}}=i \Theta^{\alpha} \sigma^{\hat{m}}{ }_{\alpha \dot{\beta}} \varepsilon^{\dot{\beta} \dot{\alpha}} & e^{\dot{\alpha} \mu}=0 & e_{\dot{\mu}}^{\dot{\alpha}}=\delta_{\dot{\mu}}^{\dot{\alpha}}\end{array}\right)$, where $\hat{a}=(a$ or $\underline{a}), a=0,1,2,3 ; \underline{a}=(+),(-)$.

The supersymmetry transformations of the component fields can be found using the differential operators. The covariant constraint $\bar{D}_{\dot{\alpha}} \Phi\left(z^{M}\right)=0$, which does not impose equations of motion on the component fields, defines the chiral superfield, $\Phi$. Under the supersymmetry transformation the chiral field transforms as follows: $\delta_{\xi} \Phi=(\xi q+\bar{\xi} \bar{q}) \times \Phi=\delta_{\xi} \underline{A}(\eta)+\sqrt{2} \theta \delta_{\xi} \chi(x)+\theta \theta \delta_{\xi} F(x)+\cdots$ in case of $Q=q$, and $\delta_{\xi} \underline{\Phi}=(\underline{\xi q}+\bar{\xi} \bar{q}) \times \underline{\Phi}=\delta_{\underline{\xi}} A(x)+\sqrt{2} \underline{\theta} \delta_{\underline{\xi}} \underline{\chi}(\eta)+\underline{\theta} \underline{\theta} \delta_{\underline{\xi}} \underline{F}(\eta)+\cdots$ in case of $Q=\underline{q}$, where as before $A(x)=\underline{A}(\bar{\eta})$. The chiral superfield contains the same component fields as the Wess-Zumino model for MS-SUSY theory. The supervolume integrals of products of superfields constructed in the superspace $\left(x^{m}, \theta, \bar{\theta}\right)$ will lead to the supersymmetric kinetic energy for the Wess-Zumino model $\int d^{4} x d^{4} \theta \Phi^{\dagger} \Phi$, where the superspace Lagrangian reads $\Phi^{\dagger} \Phi=\underline{A}^{*} \underline{A}+\cdots+\theta \theta \bar{\theta} \bar{\theta}\left[\frac{1}{4} \underline{A}^{*} \underline{\square} \underline{A}+\frac{1}{4} \underline{\underline{A}} \underline{A}^{*} \underline{A}-\right.$ $\left.\frac{1}{2} \partial_{\underline{m}} \underline{A}^{*} \partial^{\underline{m}} \underline{A}+F^{*} F+\frac{i}{2} \partial_{m} \bar{\chi} \bar{\sigma}^{m} \chi-\frac{i}{2} \bar{\chi} \bar{\sigma}^{m} \partial_{m} \chi\right]$, where $\square A=\square \underline{A}$ and $\partial_{\underline{m}} \underline{A}^{*} \partial \underline{m} \underline{A}=\bar{\partial}_{m} A^{*} \partial^{m} A$. Similarly, the supersymmetric kinetic energy for the Wess-Zumino model constructed in the superspace $(\eta \underline{\underline{m}}, \underline{\theta}, \underline{\bar{\theta}})$ for MS-SUSY theory is $\int d^{2} \eta d^{4} \underline{\theta} \underline{\Phi^{\dagger}} \underline{\Phi}$, where the superspace Lagrangian is written down $\Phi^{\dagger} \Phi=\underline{A}^{*} \underline{A}+\cdots+\theta \theta \bar{\theta} \bar{\theta}\left[\frac{1}{4} \underline{A}^{*} \underline{\underline{A}}+\frac{1}{4} \underline{\underline{A}} \underline{A}^{*} \underline{A}-\frac{1}{2} \partial_{\underline{m}} \underline{A}^{*} \partial^{\underline{m}} \underline{A}+F^{*} F+\frac{i}{2} \partial_{m} \bar{\chi} \bar{\sigma}^{m} \chi-\frac{i}{2} \bar{\chi} \bar{\sigma}^{m} \partial_{m} \chi\right]$, Тo complete the model, we also need superspace expressions for the masses and couplings, which can be easily found in analogy of the standard theory, namely: 1) fermion masses and Yukawa couplings, $\left(\partial^{2} \mathcal{P} / \partial \mathcal{A}^{2}\right) \psi \psi$; and 2) the scalar potential, $\mathcal{V}\left(\mathcal{A}, \mathcal{A}^{*}\right)=|\partial \mathcal{P} / \partial \mathcal{A}|^{2} ;$ where $\mathcal{P}=(1 / 2) m \Phi^{2}+(1 / 3) \lambda \Phi^{3}$ is the most general renormalizable interaction for a single chiral superfield. Thereby, the auxiliary field equation of motion reads $\mathcal{F}^{*}+(\partial \mathcal{P} / \partial \mathcal{A})=0$. Similarly, we can treat the vector superfields, etc. Here we shall forbear to write them out as the standard theory is so well known.

\subsection{Unaccelerated uniform motion; a foundation of SR}

Let impose peculiar constraints upon the anticommuting spinors $(\underline{\xi}, \underline{\bar{\xi}})$ and $(\xi, \bar{\xi}): \underline{\xi}^{\alpha}=i \frac{\tau}{\sqrt{2}} \underline{\theta}^{\alpha}, \quad \underline{\bar{\xi}}_{\dot{\alpha}}=$ $-i \frac{\tau^{*}}{\sqrt{2}} \bar{\theta}_{\dot{\alpha}}, \xi^{\alpha}=i \frac{\tau}{\sqrt{2}} \theta^{\alpha}, \bar{\xi}_{\dot{\alpha}}=-i \frac{\tau^{*}}{\sqrt{2}} \bar{\theta}_{\dot{\alpha}}$, and write down the infinitesimal displacement arisen in $\underline{M}_{2}$ as $\Delta \eta \underline{m}=v \underline{m} \tau=\underline{\theta} \sigma \underline{m} \underline{\bar{\xi}}-\underline{\xi} \sigma \underline{\underline{m}} \underline{\hat{\theta}}$, where the parameter $\tau\left(=\tau^{*}\right)$ can physically be interpreted as the duration time of atomic double transition of a particle from $M_{4}$ to $\underline{M}_{2}$ and back. So, $v^{(+)} \tau=$ $i\left(\underline{\theta}_{1} \bar{\xi}_{1}-\underline{\xi}_{1} \bar{\theta}_{1}\right), v^{(-)} \tau=i\left(\underline{\theta}_{2} \underline{\xi}_{2}-\underline{\xi}_{2} \bar{\theta}_{2}\right)$, and that $v^{2} \tau^{2}=v^{(+)} v^{(-)} \tau^{2}=-\left(\underline{\theta}_{1} \underline{\xi}_{1}-\underline{\xi}_{1} \underline{\theta}_{1}\right)\left(\underline{\theta}_{2} \underline{\xi}_{2}-\underline{\xi}_{2} \underline{\theta}_{2}\right)=$ $4 \underline{\theta}_{1} \underline{\bar{\theta}}_{1} \underline{\theta}_{2} \underline{\bar{\theta}}_{2} \geq 0$. Hance $v^{(+)}=\sqrt{2} \underline{\theta}_{1} \underline{\theta}_{1} \geq 0, v^{(-)}=\sqrt{2} \underline{\theta}_{2} \underline{\theta}_{2} \geq 0$. According to embedding map, therefore, we may introduce the velocity of light $(c)$ in vacuum as maximum attainable velocity for uniform motions of all the particles in the Minkowski background space, $M_{4}: c=\frac{1}{\sqrt{2}}\left(v^{(+)}+v^{(-)}\right)=$ $\sqrt{2}\left(\underline{\theta}_{1} \underline{\theta}_{1}+\underline{\theta}_{2} \underline{\theta}_{2}\right)=\sqrt{2} \underline{\theta} \underline{\bar{\theta}}=\mathrm{const}, v_{q}=\frac{1}{\sqrt{2}}\left(v^{(+)}-v^{(-)}\right)=\sqrt{2}\left(\underline{\theta}_{1} \underline{\theta}_{1}-\underline{\theta}_{2} \underline{\bar{\theta}}_{2}\right)= \pm|\vec{v}|=\mathrm{const}$, $|\vec{v}| \leq c$. The spinors $\theta(\underline{\theta}, \underline{\xi})$ and $\xi(\underline{\theta}, \xi)$ satisfy the embedding map (??), namely $\Delta q^{0}=\Delta x^{0}$ and $\Delta q^{2}=(\Delta \vec{x})^{2}$, so we have $\underline{\theta} \sigma^{0} \bar{\xi}-\underline{\xi} \sigma^{0} \underline{\bar{\theta}}=\theta \sigma^{0} \bar{\xi}-\xi \sigma^{0} \bar{\theta},\left(\underline{\theta} \sigma^{3} \underline{\bar{\xi}}-\underline{\xi} \sigma^{3} \underline{\bar{\theta}}\right)^{2}=(\theta \vec{\sigma} \bar{\xi}-\xi \vec{\sigma} \bar{\theta})^{2}$, such that $\underline{\theta}_{1} \underline{\theta}_{1}+\underline{\theta}_{2} \underline{\theta}_{2}=\underline{\theta} \underline{\bar{\theta}}=\theta \bar{\theta}, \underline{\theta}_{1} \underline{\theta}_{1}-\underline{\theta}_{2} \underline{\theta}_{2}= \pm \sqrt{\frac{3}{2}}(-\theta \theta \bar{\theta} \bar{\theta})^{1 / 2}= \pm \sqrt{\frac{3}{2}} \theta \bar{\theta}$, where we use the following relations: $\left(\theta \sigma^{m} \bar{\theta}\right)\left(\theta \sigma^{n} \bar{\theta}\right)=\frac{1}{2} \theta \theta \bar{\theta} \bar{\theta} \eta^{m n},(-\theta \theta \bar{\theta} \bar{\theta})^{1 / 2}=(\theta \bar{\theta} \theta \bar{\theta})^{1 / 2}=\theta \bar{\theta}$.So, $\underline{\theta}_{1} \underline{\theta}_{1}=\frac{1}{2}\left(1 \pm \sqrt{\frac{3}{2}}\right) \theta \bar{\theta}$, $\underline{\theta}_{2} \underline{\theta}_{2}=\frac{1}{2}\left(1 \mp \sqrt{\frac{3}{2}}\right) \theta \bar{\theta}$. Hence we conclude that the unaccelerated uniform motion of a particle in $M_{4}$ 
is encoded in the spinors $\underline{\theta}$ and $\underline{\bar{\theta}}$ referred to the master space $\underline{M}_{2}$, which is an individual companion to the particle of interest. Therefore, to account for the most important two postulates constituting a foundation of SR, it would be necessary further to impose certain constraints upon the constant Lorentz spinors $\underline{\theta}$. Lorentz invariance is a fundamental symmetry and refers to measurements of ideal inertial observers that move uniformly forever on rectilinear timelike worldlines. In view of relativity of velocity of a particle, we are of course not limited to any particular spinor $\underline{\theta}(\vec{v})$, but can choose at will any other spinors $\underline{\theta}^{\prime}\left(\overrightarrow{v^{\prime}}\right), \underline{\theta^{\prime \prime}}\left(\overrightarrow{v^{\prime \prime}}\right), \ldots$ relating respectively to velocities $\overrightarrow{v^{\prime}}, \overrightarrow{\overrightarrow{v^{\prime \prime}}}, \ldots$, whose functional dependence (transformational law) on the original spinor $\underline{\theta}(\vec{v})$ is known. Of the various possible transformations, we must consider for a validity of SR only those which obey the following constraints: 1. $\underline{\theta} \underline{\bar{\theta}}=\underline{\theta}^{\prime} \underline{\theta}^{\prime}=\underline{\theta}^{\prime \prime} \underline{\theta}^{\prime \prime}=\cdots=\frac{c}{\sqrt{2}}=\mathrm{const} ; 2 . \underline{\theta}_{1} \underline{\zeta}_{1} \underline{\theta}_{2} \underline{\zeta}_{2}=\underline{\theta}_{1}^{\prime} \underline{\zeta}_{1}^{\prime} \underline{\theta}_{2}^{\prime} \underline{\bar{\zeta}}_{2}^{\prime}=\cdots=$ inv. According to first relation, we may introduce a notion of time, for the all inertial frames of reference S, S', S",..., we have then standard Lorentz code (SLC)-relations: $x^{0}=c t, \quad x^{0^{\prime}}=c t^{\prime}, \quad x^{0^{\prime \prime}}=c t^{\prime \prime}, \ldots$ This is a second postulate of SR (Einstein's postulate) that the velocity of light $(c)$ in free space appears the same to all observers regardless the relative motion of the source of light and the observer. By virtue of second relation and equations above, we may derive the invariant interval between the two events defined in Minkowski spacetime: $8 \underline{\theta}_{1} \underline{\underline{\theta}}_{1} \underline{\theta}_{2} \underline{\bar{\theta}}_{2} \Delta t^{2}=2 v^{2} \Delta t^{2}=\left(c^{2}-v_{q}^{2}\right) \Delta t^{2}=\left(c^{2}-\vec{v}^{2}\right) \Delta t^{2}=$ $c^{2} \Delta t^{2}-\Delta \vec{x}^{2} \equiv \Delta s^{2}=8 \underline{\theta}_{1}^{\prime} \underline{\theta}_{1}^{\prime} \underline{\theta}_{2}^{\prime} \underline{\theta}_{2}^{\prime} \Delta t^{\prime 2}=c^{2} \Delta t^{\prime 2}-\Delta \vec{x}^{\prime 2} \equiv \Delta s^{\prime 2}=\cdots=i n v$, where we introduce the physical finite time interval, $\Delta t=k \tau$, between two events as integer number of the duration time, $\tau$, of atomic double transition of a particle from $M_{4}$ to $\underline{M}_{2}$ and back, where $k$ is the number of double transformations. Hence, an unaccelerated uniform motion, for example, of spin-0 particle in $M_{4}$ can be described by the chiral superfield $\underline{\Phi}\left(\eta^{\hat{m}}, \underline{\theta}, \underline{\bar{\theta}}\right)$, while a similar motion of spin-1/2 particle in $M_{4}$ can be described by the chiral superfield $\Phi\left(x^{m}, \theta, \bar{\theta}\right)$, etc. So, we may refer to all constant Lorentz spinors as the SLC-spinors, which constitute a foundation of SR. Hence, in view of the MS-SUSY mechanism of motion, the uniform motion of a particle in $M_{4}$ is encoded in the spinors $\underline{\theta}$ and $\underline{\hat{\theta}}$, which refer to $\underline{M}_{2}$. This will call for a complete reconsideration of our ideas of Lorentz motion code, to be now referred to as the individual code of a particle, defined as its intrinsic property.

\subsection{Extended supersymmetry and ELC}

In four dimensions, it is possible to have as many as eight supersymmetries: $N_{\max }=4$ for renormalizable flat-space field theories; $N_{\max }=8$ for consistent theories of supergravity. It has been shown that the $\mathrm{N}=4$ theory is not only renormalizable but actually finite. So, the theories with $N>1$ may play a key role in high-energy physics. These models explore more than one distinct copy of the supersymmetry generators, $Q_{\alpha i}$, therefore, this perspective ultimately requires to relax the Einstein's postulate, because it is natural now to circumvent the limitations to any particular spinor $\underline{\theta}$, instead, considering $i(=1, \ldots, 4)$-th $\left(N_{\max }=4\right)$ copy of the spinors $\Theta^{\alpha i} \equiv\left(\theta^{\alpha i}\right.$ or $\left.\underline{\theta}^{\alpha i}\right)$. Therefore, we now have a straightforward generalization: 1. $\underline{\theta}^{i} \underline{\bar{\theta}}^{i}=\underline{\theta}^{i \prime} \underline{\bar{\theta}}^{i \prime}=\underline{\theta}^{i \prime \prime} \underline{\bar{\theta}}^{i \prime \prime}=\cdots=\frac{c_{i}}{\sqrt{2}}=$ const; $\quad$ (no sum over i), 2. $\underline{\theta}_{1}^{i} \underline{\zeta}_{1}^{i} \underline{\theta}_{2}^{i} \underline{\zeta}_{2}^{i}=\underline{\theta}^{i \prime}{ }_{1} \underline{\zeta}^{i \prime}{ }_{1} \underline{\theta}^{i \prime}{ }_{2} \underline{\zeta}^{i \prime}{ }_{2}=\cdots=i n v$. This observation allows us to lay forth the extension of Lorentz code, at which SLC violating new physics appears. We may now consider the particles of $i(=1, \ldots, 4)$-th type $\left(N_{\max }=4\right)$. That is to say, the $i$-th type particle in free Minkowski space carries an individual Lorentz motion code with its own maximum attainable velocity $c_{i}$, i.e., its own velocity of 'light-like' state: $c_{i}=\frac{1}{\sqrt{2}}\left(v_{i}^{(+)}+v_{i}^{(-)}\right)=\sqrt{2}\left(\underline{\theta}_{1 i} \underline{\bar{\theta}}_{1 i}+\underline{\theta}_{2 i} \underline{\bar{\theta}}_{2 i}\right)=\sqrt{2} \underline{\theta}_{i} \underline{\bar{\theta}}_{i}=$ const, (no sum over i), $v_{q i}=\frac{1}{\sqrt{2}}\left(v_{i}^{(+)}-v_{i}^{(-)}\right)=\sqrt{2}\left(\underline{\theta}_{1 i} \underline{\theta}_{1 i}-\underline{\theta}_{2 i} \underline{\theta}_{2}\right)= \pm\left|\vec{v}_{i}\right|=$ const, $\left|\vec{v}_{i}\right| \leq c_{i}$. A general solution to the Lorentz covariance in the theory can be easily accommodated if the 'time' at which event occurs is extended by allowing an extra dependence on 'different type' readings $t_{i}$ referred to the particles of different type. They satisfy for all inertial frames of reference S, S', S', ,.., so-called 'ELC-relations': $x^{0} \equiv c_{1} t_{1}=\cdots=c_{i} t_{i}=\ldots, x^{0^{\prime}} \equiv c_{1} t_{1}^{\prime}=\cdots=c_{i} t_{i}^{\prime}=\ldots$, where $c_{1} \equiv c$ is the speed of light in vacuum, and $c_{i}>c_{1},(i=2,3,4)$ are speeds of the additional 'light-like' states, higher than that of light. The clock reading $t_{i}$ can be used for the $i$-th type particle, the velocity of which reads $v_{i}=x / t_{i}=c_{i} x / x^{0}$, so $\beta=v_{1} / c_{1}=\ldots v_{i} / c_{i}=\cdots \equiv v / c=x / x^{0}$. If $v_{i}=c_{i}$ then $v_{1}=c_{1}$, and the proper time of 'light-like' states are described by the null vectors $d s_{1}^{2}=\ldots d s_{i}^{2}=\cdots=0$. The extended Lorentz transformation equations for given $i$-th and $j$-th type clock readings can be written then in the form 
A new look at some aspects of geometry, particle physics, inertia, radiation and cosmology

$x^{\prime}=\gamma(x-v t), \quad t_{i}^{\prime}=\gamma \frac{c_{j}}{c_{i}}\left(t_{j}-\frac{v_{j}}{c_{j}^{2}} x\right), \quad y^{\prime}=y, \quad z^{\prime}=z, \quad \gamma \equiv 1 / \sqrt{1-\beta^{2}}$. Hence, like the standard SR theory, regardless the type of clock, a metre stick traveling with system $\mathrm{S}$ measures shorter in the same ratio, when the simultaneous positions of its ends are observed in the other system $\mathrm{S}^{\prime}: d x^{\prime}=d x / \gamma$. Furthermore, a time interval $d t_{i}$ specified by the $i$-th type readings, which occur at the same point in system $\mathrm{S}(d x=0)$, will be specified with the $j$-th type readings of system $\mathrm{S}^{\prime}$ as $d t_{j}^{\prime}=\gamma\left(c_{i} / c_{j}\right) d t_{i}$. Here we have called attention to the fact that the mere composition of velocities which are not themselves greater than that of $c_{i}$ will never lead to a speed that is greater than that of $c_{i}$. Inevitably in the ELC-framework a specific task is arisen then to distinguish the type of particles. This evidently cannot be done when the velocity ranges of different type particles intersect. To reconcile this situation, we note that, we may freely interchange the types of particles in the intersection. Therefore, we adopt following convention. With no loss of generality, we may re-arrange a general solution that the particles with velocities $v_{1}<c_{1}$, regardless their type, will be treated as the 1-th type particles and, thus, a common clock reading for them and light will be set as $t_{1}$. This part of a formalism is completely equivalent to the SLC-framework. Successively, the particles, other than 'light-like' ones, with velocities in the range $c_{i-1} \leq v_{i}<c_{i}$, regardless their type, will be treated as the $\mathrm{i}$-th type particles and, thus, a common clock reading for them and 'light-like' state $(i)$ will be set as $t_{i}$. The invariant momentum $p_{i}^{2}=p_{\mu i} p_{i}^{\mu}=\left(\frac{E_{i}}{c_{i}}\right)^{2}-\vec{p}_{i}^{2}=m_{0 i}^{2} c_{i}^{2}=p_{1}^{2}=p_{\mu 1} p_{1}^{\mu}=\left(\frac{E_{1}}{c_{1}}\right)^{2}-\vec{p}_{1}^{2}=m_{0}^{2} c_{1}^{2}$, introduces a modified dispersion relation for $i$-th type particle: $E_{i}^{2}=\vec{p}_{i}^{2} c_{i}^{2}+m_{0 i}^{2} c_{i}^{4}=\vec{p}_{i}^{2} c_{i}^{2}+m_{01}^{2} c_{1}^{2} c_{i}^{2}$, where the mass of $i$-th type particle has the value $m_{0 i}$, when at rest, the positive energy is $E_{i}=m_{i} c_{i}^{2}=\gamma m_{0 i} c_{i}^{2}=\gamma m_{01} c_{1} c_{i}$, and $\vec{p}_{i}=m_{i} \vec{v}_{i}=\gamma m_{0 i} \vec{v}_{i}$ is the momentum. This relation modifies the well-known Einstein's equation that energy $E$ always has immediately associated with it a positive mass $m_{i}=\gamma m_{0 i}$, when moving with the velocity $\vec{v}_{i}$. Having set this theoretical background, one may find some consequences for the superluminal propagation of particles. In particular, in the ELC-framework of uniform motion, the time elapsing between the cause $t_{i A}$ and its effect $t_{i B}$ as measured for the $i$-th type superluminal particle is $\Delta t_{i}=t_{i B}-t_{i A}=\frac{x_{B}-x_{A}}{v_{i}}$, where $x_{A}$ and $x_{B}$ are the coordinates of the two points $\mathrm{A}$ and B. In another system $\mathrm{S}^{\prime}$, which is chosen as before and has the arbitrary velocity $V \equiv V_{j}$ with respect to S, the time elapsing between cause and effect would be $\Delta t_{i}^{\prime}=\frac{1-\frac{V_{j}}{c_{j}} \frac{v_{i}}{c_{i}}}{\sqrt{1-\frac{V_{j}^{2}}{c_{j}^{2}}}} \Delta t_{i} \geq 0$, where $t_{i B}=\left(c_{j} / c_{i}\right) t_{j B}$ and $t_{i A}=\left(c_{j} / c_{i}\right) t_{j A}$. That is, the ELC-framework recovers the causality for a superluminal propagation, so the starting of the superluminal impulse at A and the resulting phenomenon at B are being connected by the relation of cause and effect in arbitrary inertial frames. Furthermore, in this framework, we may give a justification of forbiddance of Vavilov-Cherenkov radiation/or analog processes in vacuum. Thereby, in this framework we have to set, for example, $k_{1}=\left(\frac{\omega}{c_{1}}, \overrightarrow{k_{1}}\right)$ for the 1-th type $\gamma_{1}$ photon, provided $\overrightarrow{k_{1}}=\vec{e}_{k} \frac{\omega}{c_{1}}$, and $p_{2}=\left(\frac{E_{2}}{c_{2}}, \overrightarrow{p_{2}}\right)$ for the 2-nd type superluminal particle. Then the process $\left(l_{2} \rightarrow l_{2}+\gamma_{1}\right)$ becomes kinematically permitted if and only if $k_{1} p_{2}=\frac{\omega}{c_{1}} \frac{E_{2}}{c_{2}}\left(1-\vec{e}_{k} \frac{\vec{v}_{2}}{c_{2}}\right)=0$, which yields $\omega \equiv 0$ because of $\left(1-\vec{e}_{k} \frac{\vec{v}_{\nu 2}}{c_{2}}\right) \neq 0$. This evades constraints due to VC-like processes since the superluminal particle $\nu_{\mu 2}$ does not actually travel faster than the speed $c_{2}$. Finally, in ELC-framework we discuss the VC-radiation of the charged superluminal particle propagating in vacuum with a constant speed $v_{2}>c_{1}$ higher than that of light. Recall that, for a charged particle $(e \neq 0)$ moving in a transparent, isotropic and non-magnetic medium with a constant velocity higher than velocity of light in this medium the VC radiation is allowed. The energy loss per frequency is $d F=-d \omega \frac{i e^{2}}{2 \pi} \sum \omega\left(\frac{1}{c^{2}}-\frac{1}{\varepsilon v^{2}}\right) \int \frac{d \zeta}{\zeta}$, where the direction of the velocity $\vec{v}$ is chosen to be $x$-direction: $k_{x}=k \cos \theta=\omega / v, k=n \omega / c$ is the wave number $n=\sqrt{\varepsilon}$ is the real refractive index, $\varepsilon$ is the permittivity. The summation is over terms with $\omega= \pm|\omega|$, and a variable $\zeta=q^{2}-\omega^{2}\left(\frac{\varepsilon}{c^{2}}-\frac{1}{v^{2}}\right)$ is introduced, provided $q=\sqrt{k_{y}^{2}+k_{z}^{2}}$. The integrand is strongly peaked near the singular point $\zeta=0$, for which $q^{2}+k_{x}^{2}=k^{2}$. Using standard technique, it can be easily transformed to be applicable in ELC-framework for the charged superluminal particle of 2 -nd type propagating in vacuum (i.e. if $\varepsilon=1$ ) with a constant speed $v_{2}$ higher than that of light $\left(c_{1} \leq v_{2}<c_{2}\right): d F=-d \omega \frac{i e^{2}}{2 \pi} \sum \omega\left(\frac{1}{c_{2}^{2}}-\frac{1}{v_{2}^{2}}\right) \int \frac{d \zeta}{\zeta}$. Hence $\zeta=q_{1}^{2}-\omega^{2}\left(\frac{1}{c_{2}^{2}}-\frac{1}{v_{2}^{2}}\right)$, where $q_{1}=\sqrt{k_{y 1}^{2}+k_{z 1}^{2}}, q_{1}^{2}+k_{x 1}^{2}=k_{1}^{2}=\omega^{2} / c_{1}^{2}$, and now $k_{x 1} v_{2}=\omega$. We have then $\zeta=\frac{\omega^{2}}{c_{2}^{2}}\left(\frac{c_{2}^{2}}{v_{2}^{2} \cos ^{2} \theta}-1\right) \neq 0$, because of $v_{2}<c_{2}$, and that the integral is zero, since the integrand has no poles. Hence, as expected, 


\subsection{Accelerated motion and local MS-SUSY}

In case of an accelerated $(a=|\vec{a}| \neq 0)$ motion of a particle in $M_{4}$, we have then $\frac{i}{\sqrt{2}}\left(\underline{\theta} \sigma^{3} \frac{d^{2} \bar{\xi}}{d t^{2}}-\right.$ $\left.\frac{d^{2} \xi}{d t^{2}} \sigma^{3} \underline{\bar{\theta}}\right)=\frac{d^{2} q}{d t^{2}}=a=\frac{1}{\sqrt{2}}\left(\frac{d^{2} \eta^{(+)}}{d t^{2}}-\frac{d^{2} \eta^{(-)}}{d t^{2}}\right)=\frac{1}{\sqrt{2}}\left(a^{(+)}-a^{(-)}\right), a^{( \pm)}=\frac{d v^{( \pm)}}{d t}$. So, we may relax the condition $\partial_{\hat{m}} \epsilon=0$ and promote this symmetry to a local supersymmetry in which the parameter $\epsilon=\epsilon\left(X^{\hat{m}}\right)$ depends explicitly on $X^{\hat{m}}$. Such a local SUSY can already be read off from the considered above algebra in the form $[\epsilon(X) Q, \bar{Q} \bar{\epsilon}(X)]=2 \epsilon(X) \sigma^{\hat{m}} \bar{\epsilon}(X) p_{\hat{m}}$, which says that the product of two supersymmetry transformations corresponds to a translation in space-time of which the four momentum $p_{\hat{m}}$ is the generator. Similar to the results of subsection $F$, the multiplication of two local successive transformations induces the motion $g(0, \epsilon(X), \bar{\epsilon}(X)) \Omega\left(X^{\hat{m}}, \Theta, \bar{\Theta}\right) \longrightarrow$ $\left(X^{\hat{m}}+i \Theta \sigma^{\hat{m}} \bar{\epsilon}(X)-i \epsilon(X) \sigma^{\hat{m}} \bar{\Theta}, \Theta+\epsilon(X), \bar{\Theta}+\bar{\epsilon}(X)\right)$, and, in accord, the transformation is expected to be somewhat of the form $\left[\delta_{\epsilon_{1}(X)}, \delta_{\epsilon_{2}(X)}\right] V \sim \epsilon_{1}(X) \sigma^{\hat{m}} \bar{\epsilon}_{2}(X) \partial_{\hat{m}} V$, that differ from point to point, namely this is the notion of a general coordinate transformation. Whereupon we see that for the local MS-SUSY to exist it requires the background spaces $\left(\underline{M}_{2}, \widetilde{M}_{4}\right)$ to be curved. Thereby, the space $\widetilde{M}_{4}$, in order to become on the same footing with the distorted space $\underline{\widetilde{M}}_{2}$, refers to the accelerated proper reference frame of a particle, without relation to other matter fields. A useful guide in the construction of local superspace is that it should admit rigid superspace as a limit. The reverse is also expected, since if one starts with a constant parameter $\epsilon$ and performs a local Lorentz transformation, then this parameter will in general become space-time dependent as a result of this Lorentz transformation. The mathematical structure of the local MS-SUSY theory has much in common with those used in the geometrical framework of standard supergravity theories. In its simplest version, supergravity was conceived as a quantum field theory whose action included the Einstein-Hilbert term, where the graviton coexists with a fermionic field called gravitino, described by the Rarita-Scwinger kinetic term. The two fields differ in their spin: 2 for the graviton, $3 / 2$ for the gravitino. The different $4 \mathrm{D} N=1$ supergravity multiplets all contain the graviton and the gravitino, but differ by their systems of auxiliary fields. These fields would transform into each other under local supersymmetry. We may use the usual language which is almost identical to the vierbien formulation of GR with some additional input. In this framework supersymmetry and general coordinate transformations are described in a unified way as certain diffeomorphisms. The motion generates the super-general coordinate reparametrization $z^{M} \longrightarrow z^{M}=z^{M}-\zeta^{M}(z)$, where $\zeta^{M}(z)$ arc arbitrary functions of $z$. The dynamical variables of superspace formulation are the frame field $E^{A}(z)$ and connection $\Omega$. The superspace $\left(z^{M}, \Theta, \bar{\Theta}\right)$ has at each point a tangent superspace spanned by the frame field $E^{A}(z)=d z^{M} E_{M}^{A}(z)$, defined as a 1-form over superspace, with coefficient superfields, generalizing the usual frame, namely supervierbien $E_{M}^{A}(z)$. Here, we use the first half of capital Latin alphabet $A, B, \ldots$ to denote the anholonomic indices related to the tangent superspace structure group, which is taken to be just the Lorentz group. The formulation of supergravity in superspace provides a unified description of the vierbein and the Rarita-Schwinger fields. They are identified in a common geometric object, the local frame $E^{A}(z)$ of superspace. Covariant derivatives with respect to local Lorentz transformations are constructed by means of the spin connection, which is a 1-form in superspace as well. Here we shall forbear to write the details out as the standard theory is so well known. The supervierbien $E_{M}^{A}$ and spin- connection $\Omega$ contain many degrees of freedom. Although some of these are removed by the tangent space and supergeneral coordinate transformations, there still remain many degrees of freedom. There is no general prescription for deducing necessary covariant constraints which if imposed upon the superfields of super-vierbien and spin-connection will eliminate the component fields. However, some usual constraints can be found using tangent space and supergeneral coordinate transformations of the torsion and curvature covariant tensors, given in appropriate supergauge. Together with other details of the theory, they can be seen in the textbooks. The final form of transformed super-vierbien, can be written as $\left.E_{A}{ }^{M}(z)\right|_{\Theta=\bar{\Theta}=0}=\left(\begin{array}{cll}e_{\hat{m}}^{\hat{a}}(X) & \frac{1}{2} \psi_{\hat{m}}^{\alpha}(X) & \frac{1}{2} \bar{\psi}_{\hat{m} \dot{\alpha}}(X) \\ 0 & \delta_{\alpha}^{\mu} & 0 \\ 0 & 0 & \delta_{\dot{\alpha}}^{\mu}\end{array}\right)$, where the fields of graviton $e_{\hat{m}}^{\hat{a}}$ and gravitino $\frac{1}{2} \psi_{\hat{m}}^{\alpha}, \frac{1}{2} \bar{\psi}_{\hat{m} \dot{\alpha}}$ cannot be gauged away. Provided, we 
A new look at some aspects of geometry, particle physics, inertia, radiation and cosmology

have $e_{\hat{a}}^{\hat{m}} e_{\hat{m}}^{\hat{b}}=\delta_{\hat{a}}^{\hat{b}}, \psi_{\hat{a}}^{\mu}=e_{\hat{a}}^{\hat{m}} \psi_{\hat{m}}^{\alpha} \delta_{\alpha}^{\mu}, \bar{\psi}_{\hat{a} \dot{\mu}}=e_{\hat{a}}^{\hat{m}} \bar{\psi}_{\hat{m} \dot{\alpha}} \delta_{\mu}^{\dot{\alpha}}$. The tetrad field $e_{\hat{m}}^{\hat{a}}(X)$ plays the role of a gauge field associated with local transformations. The Majorana type field $\frac{1}{2} \psi_{\hat{m}}^{\alpha}$ is the gauge field related to local supersymmetry. These two fields belong to the same supergravity multiplet which also accommodates auxiliary fields so that the local supersymmetry algebra closes. Under infinitesimal transformations of local supersymmetry, they transformed as $\delta e_{\hat{m}}^{\hat{a}}=i\left(\psi_{\hat{m}} \sigma^{\hat{a}} \zeta-\zeta \sigma^{\hat{a}} \bar{\psi}_{\hat{m}}\right)$, $\delta \psi_{\hat{m}}=-2 \mathcal{D}_{\hat{m}} \zeta^{\alpha}+i e_{\hat{m}}^{\hat{c}}\left\{\frac{1}{3} M\left(\varepsilon \sigma_{\hat{c}} \bar{\zeta}\right)^{\alpha}+b_{\hat{c}} \zeta^{\alpha}+\frac{1}{3} b^{\hat{d}}\left(\zeta \sigma_{\hat{d}} \bar{\sigma}_{\hat{c}}\right)\right\}$, etc., where $M_{4}$ and $b_{\bar{a}}$ are the auxiliary fields, and $\zeta^{\alpha}(z)=\zeta^{\alpha}(X), \quad \bar{\zeta}^{\alpha}(z)=\bar{\zeta}^{\alpha}(X)$ and $\zeta^{\bar{a}}(z)=2 i\left[\Theta \sigma^{\hat{a}} \bar{\zeta}(X)-\zeta(X) \sigma^{\hat{a}} \bar{\Theta}\right]$. The chiral superfields are defined as $\overline{\mathcal{D}}_{\dot{\alpha}} \Phi=0$, therefore, the components fields are $\mathcal{A}=\left.\Phi\right|_{\Theta=\bar{\Theta}=0}, \psi_{\alpha}=\left.\frac{1}{\sqrt{2}} \mathcal{D}_{\alpha} \Phi\right|_{\Theta=\bar{\Theta}=0}$, $\mathcal{F}=-\left.\frac{1}{4} \mathcal{D}^{\alpha} \mathcal{D}_{\alpha} \Phi\right|_{\Theta=\bar{\Theta}=0}$, which carry Lorentz indices. Under infinitesimal transformations of local supersymmetry, they transformed as $\delta \mathcal{A}=-\sqrt{2} \zeta^{\alpha} \psi_{\alpha}, \delta \psi_{\alpha}=-\sqrt{2} \zeta_{\alpha} \mathcal{F}-i \sqrt{2} \sigma_{\alpha \dot{\beta}}{ }^{\hat{\zeta}} \bar{\zeta}^{\dot{\beta}} \hat{\mathcal{D}}_{\hat{a}} \mathcal{A}, \delta \mathcal{F}=$ $-\frac{1}{3} \sqrt{2} M^{*} \zeta^{\alpha} \psi_{\alpha}+\bar{\zeta}^{\dot{\alpha}}\left(\frac{1}{6} \sqrt{2} b_{\alpha \dot{\alpha}} \psi^{\dot{\alpha}}-i \sqrt{2} \hat{\mathcal{D}}_{\alpha \dot{\alpha}} \psi^{\alpha}\right)$, where $\hat{\mathcal{D}}_{\hat{a}}$ is, so-called, super-covariant derivative $\hat{\mathcal{D}}_{\hat{a}} \mathcal{A} \equiv e_{\hat{a}}{ }^{\hat{m}}\left(\partial_{\hat{m}} \mathcal{A}-\frac{i}{\sqrt{2}} \psi_{\hat{m}}^{\mu} \psi_{\mu}\right), \hat{\mathcal{D}}_{\hat{a}} \psi_{\alpha}=e_{\hat{a}}{ }^{\hat{m}}\left(\mathcal{D}_{\hat{m}} \psi_{\alpha}-\frac{1}{\sqrt{2}} \psi_{\hat{m} \alpha} \mathcal{F}-\frac{i}{\sqrt{2}} \bar{\psi}_{\hat{m}} \dot{\beta}^{\hat{\mathcal{D}}}{ }_{\alpha \dot{\beta}} \mathcal{A}\right)$. The graviton and the gravitino form thus the basic multiplet of local MS-SUSY, and one expects the simplest locally supersymmetric model to contain just this multiplet. The spin 3/2 contact term in total Lagrangian arises from equations of motion for the torsion tensor, and that the original Lagrangian itself takes the simpler interpretation of a minimally coupled spin $(2,3 / 2)$ theory.

\subsection{Inertial effects}

We would like to place the emphasis on the essential difference arisen between the standard supergravity theories and some rather unusual properties of local MS-SUSY theory. In the framework of the standard supergravity theories, as in GR, a curvature of the space-time acts on all the matter fields. The source of graviton is the energy-momentum tensor of matter fields, while the source of gravitino is the spin-vector current of supergravity. In the local MS-SUSY theory, unlike the supergravity, a curvature of space-time arises entirely due to the inertial properties of the Lorentz-rotated frame of interest, i.e. a fictitious gravitation which can be globally removed by appropriate coordinate transformations. This refers to the particle of interest itself, without relation to other matter fields. The only source of graviton and gravitino, therefore, is the acceleration of a particle, because the MS-SUSY is so constructed as to make these two particles just as being the two bosonic and fermionic states of a particle of interest in the curved background spaces $\widetilde{M}_{4}$ and $\underline{M}_{2}$, respectively, or vice versa. Whereas, in order to become on the same footing with the distorted space $\underline{M}_{2}$, the space $\widetilde{M}_{4}$ refers only to the accelerated proper reference frame of a particle. With these physical requirements, a standard Lagrangian consisted of the classical Einstein-Hilbert Lagrangian plus a part which contains the Rarita-Schwinger field and coupling of supergravity with matter superfields evidently no longer holds. Instead we are now looking for an alternative way of implications of local MS-SUSY in the model of accelerated motion and inertial effects. For example, we may with equal justice start from the reverse, which as we mentioned before is also expected. If one starts with a constant parameter $\epsilon$ and performs a local Lorentz transformation, which can only be implemented if MS and space-time are curved (deformed/distorted) $\left(\widetilde{M}_{2}, \widetilde{M}_{4}\right)$, then this parameter will in general become space-time dependent as a result of this Lorentz transformation, which readily implies local MS-SUSY. In going into practical details of the realistic local MS-SUSY model, it remains to derive the explicit form of the vierbien $e_{\hat{m}}^{\hat{a}}(\varrho) \equiv\left(e_{m}^{a}(\varrho), e_{\underline{m}}^{\underline{a}}(\varrho)\right)$, which describes fictitious graviton as a function of local rate $\varrho(\eta, m, f)$ of instantaneously change of the velocity $v^{( \pm)}$of massive $(m)$ test particle under the unbalanced net force $(f)$. At present, unfortunately, we cannot offer a straightforward recipe for deducing the alluded vierbien $e_{\hat{m}}^{\hat{a}}(\varrho)$ in the framework of quantum field theory of MS-supergravity. However, in previous section, it was described in the framework of classical physics. Together with other usual aspects of the theory, this illustrates a possible solution to the problems of inertia behind spacetime deformations. Thereby it was argued that a deformation/(distortion of local internal properties) of $\underline{M}_{2}$ is the origin of inertia effects that can be observed by us. Consequently, the next member of the basic multiplet of local MS-SUSY -fictitious gravitino, $\psi_{\hat{m}}^{\alpha}(\varrho)$, will be arisen under infinitesimal transformations of local supersymmetry, provided by the local parameters $\zeta^{M}(a)$. 


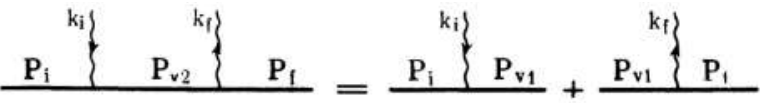

(b)

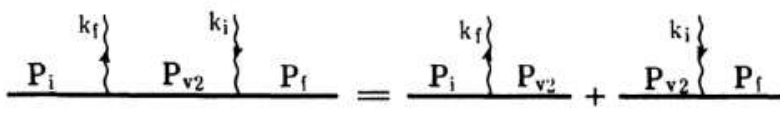

(c)

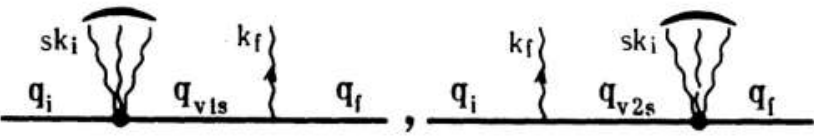

(d)

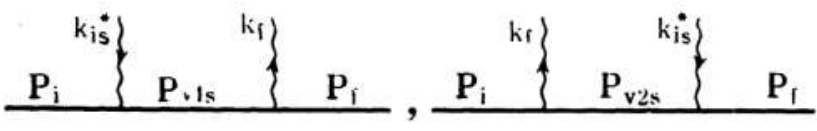

Figure 1. The amplitude of Compton scattering of a photon by an electron is represented by the sum of two amplitudes. The panels (a) and (b) stand for the case of weak and moderate radiation intensity $\xi_{i}^{2} \ll 1$; The panels (c) and (d) stand for the case of intense radiation $\xi_{i}^{2} \gtrsim 1$.

\section{Einstein's transition coefficients for Compton scattering}

The processes of interaction of electrons with intense radiation are of vital interest for interpretation of non-stationary phenomena occurring in a number of astrophysical objects. Then it is important to carry out a detailed analysis of nonlinear processes occurring at intense radiation that play a decisive role in the formation of the physical characteristics of these phenomena. Recently, with the increasing interest in non-stationary nonthermal phenomena occurring in recently discovered very interesting astrophysical objects, the research with the use of mechanisms of electron-photon interaction in cosmic plasma is being carried out more and more often and intensively. The Compton scattering is the $s$ channel of the photon-electron interaction, while the processes of annihilation and creation of electronpositron pairs is the $t$-channel. These processes of interactions are met both in weak, moderate, and in very intense radiation fields with the thermal and relativistic electrons.

The introduction of Einstein's transition coefficients is one of the outstanding events in the history of physics. There is no surprise that Milne's (see e.g. (Mihalas, 1978)) very important generalization of Einstein's relations to bound-free processes (photonionization) was dictated by the interests of astrophysical problems. Later, using the formal introduction of the indicated coefficients, by Pauli (Pauli, 1923) taken into account those quantum-theoretical properties of radiation, which in the wave theory are manifested in the form of interference fluctuations. A next important step was taken by Einstein and Ehrenfest (Einstein \& Ehrenfest, 1923), by a clear separation of absorption and emission processes in the Compton scattering of quanta by molecules. However, for a deeper understanding of these processes, it needs further detailed analysis. In the papers (Ter-Kazarian, 1984c,e) Einstein's ideas are further developed for free-virtual, virtual-free and free-free transitions for electron-photon scattering at arbitrary intense radiation by splitting Compton scattering into two components.

To start with, we consider at first the case of weak and moderate radiation intensity $\xi_{i}^{2} \ll 1$, where $\xi_{i}$ is the parameter of intensity of radiation field of initial photons $\xi_{i}^{2}=\frac{e^{2} h n_{\gamma}}{\pi m_{e}^{2} c^{2} \nu_{i}}, n_{\gamma}$ is the density of photons, and $\nu_{i}$ is their frequency. Let the electron-photon gas be in thermodynamic equilibrium. Meanwhile the distribution of electrons will be Maxwellian, and radiation will be Planckian. The amplitude of scattering of a photon by an electron is represented by the sum of two amplitudes $M=M_{1}+M_{2}$. The amplitude $M_{1}$ corresponds to the process of scattering of a photon with four momenta $k_{i}$ by an electron with four momenta $p_{i}$. After the act, they acquire impulses $k_{f}$ and $p_{f}$. This act can be represented as the sum of two constituent processes: 1$)$ the $(i \rightarrow v)$ transition of an electron from the initial free state $(i)$ to the virtual $(v)$ by absorbing the initial quantum; and 2) the transition $(v \rightarrow f)$ of an electron from the virtual state to the free state $(f)$ by emission of a finite quantum. The corresponding Feynman diagram will have the form of panels $(a, b)$ in Fig. 1 . Note the following, it is unimportant that an electron can only have discrete states or energy values. If the density of states is a continuous function in the phase space, then we have the right to replace 
these states with equiprobable, infinitesimal regions of states, between which a radiative transition is possible. Note also that when considering radiative transitions in molecules (Einstein \& Ehrenfest, 1923), the 'internal state' of the molecule itself did not play any role. Although the virtual electron is 'unusual' (it was located outside the mass shell), without addressing questions about its 'internal state', we have the right to base this study on two quantum-theoretical hypotheses: 1) electrons can make free-virtual and virtually-free transitions both under the action of radiation field (induced) and spontaneous one; 2) at thermodynamic equilibrium, the principle of detailed equilibrium can also be applied to processes, where one of the states is virtual, i.e. any process, among those in the same frequency range and direction, is compensated by the opposite process. The simplicity of hypotheses, generality and ease of further consideration, as well as a natural transition to a well-known Pauli's hypothetical statistical law (Pauli, 1923) allows us to judge their correctness. We continue with two basic equations of the conservation law for the mean transitions for the states $(i)$ and $(f)$. Then, excluding the populations of the 'internal states', we obtain the conditions of detailed equilibrium for the transitions $\left(i_{1} \rightarrow f_{1}, \quad\left(i_{2} \rightarrow f_{2}\right.\right.$ and $\left(i \rightarrow f\right.$. Through the notation $B_{i f}=B_{i_{1} v_{1}} B_{v_{1} f_{1}}+B_{i_{2} v_{2}} B_{v_{2} f_{2}}$, $B_{f i}=B_{f_{1} v_{1}} B_{v_{1} i_{1}}+B_{f_{2} v_{2}} B_{v_{2} i_{2}}$ - for induced coefficients; and $A_{i f}=B_{i_{1} v_{1}} A_{v_{1} f_{1}}+A_{i_{2} v_{2}} B_{v_{2} f_{2}}, A_{f i}=$ $B_{f_{1} v_{1}} A_{v_{1} i_{1}}+A_{f_{2} v_{2}} B_{v_{2} i_{2}}$ - for spontaneous coefficients, the coefficients of probabilities of 'real' (free-free) transitions for processes $\left(i \rightarrow f\right.$ are directly introduced, where $N_{i} / 2=N_{i_{1}}=N_{i_{2}}, N_{f} / 2=N_{f_{1}}=N_{f_{2}}$ (since the weights of the amplitudes $M_{1}$ and $M_{2}$ are the same), $n(\nu)=\frac{c^{2}}{2 h \nu^{3}} \rho(\nu)$ is the number of fillings of the quanta of frequency $\nu$. When considering the limiting case $T \rightarrow \infty$, it is easy to obtain relations between the coefficients of induced transitions. Finally, based on the fact that the energy 'absorbed' during the transition $(i \rightarrow f$ (in a unit volume of the phase space for the time $\triangle t$ ) can be represented as using the introduced coefficients, and by means of the usual quantummechanical scattering probability $W$, one can obtain the remaining relations between the coefficients of the transition probabilities. Similar reasoning for the 'radiated' energy in the transition $(f \rightarrow i$ gives additional relations. It should be noted that, in contrast to the coefficients of the probabilities of atomic transitions, here the values of the introduced coefficients are well defined, since the scattering probability $W$ is well known.

Radiation transfer equation for nonequilibrium processes. Determining the probability coefficients of transitions in the state of thermodynamic equilibrium, it is easy to turn to the radiation transfer equation for nonequilibrium processes. For example, for free-free transitions, the corresponding equation has the form $\left(\frac{1}{c} \frac{\partial}{\partial t}+\frac{\partial}{\partial l}\right) I_{i}=\int d \tau_{i} \int \frac{d \omega_{f}}{4 \pi} \delta\left[-N_{i} I_{i}\left(B_{i f} I_{f}+A_{i f}\right)+N_{f} I_{f}\left(B_{f i} I_{i}+A_{f i}\right)\right]$, where $d \tau_{i}$ is the element of the phase volume of the initial electrons.

\subsection{Interaction of electrons with the intense radiation: 'diagonal' interaction}

Next, we consider the general problem of interaction of electrons with the intense radiation $\xi_{i}^{2} \gtrsim 1$ via $s$-photon Compton scattering $s \gamma+e \rightarrow \gamma^{\prime}+e^{\prime}$. Here it is necessary to highlight two type of interaction: c) 'diagonal', when an electron absorbs these $s$-quanta from one wave; d) 'non-diagonal', when the absorbed set of $s$-quanta includes all kinds of combinations of quanta from different waves. The corresponding diagram for 'diagonal' interaction will have the form of panel $(c)$ in Fig. 1 with four conservation laws: $q_{i}+s h k_{i}=q_{f}+h k_{f}$, where $q_{i}^{\mu}=p_{i}^{\mu}+\frac{m_{e}^{2} c^{4} \xi_{i}^{2}}{2 k_{i} p_{i}} k_{i}^{\mu}$, and $p_{i}^{\mu}$ is the four-momentum of an electron at time $t=-\infty$. If we introduce a new concept of 'effective photon' with fourmomentum $k_{i s}^{*}=s^{*} k_{i}$ where $s^{*} \equiv s+\frac{m_{e}^{2} c^{4} \xi_{i}^{2}}{2}\left(\frac{1}{k_{i} p_{i}}-\frac{1}{k_{f} p_{f}}\right)$, then instead of $s$-photon scattering by electron with an effective four-momentum $q_{f}$, with equal footing we should consider the scattering of one 'effective photon' by free electron. Thereby the diagram $(c)$ should be replaced with the $(d)$ in Fig. 1. Consequently, the problem is wholly reduced to the above considered case of one-photon scattering. In this case, it should be assumed that the detailed balance condition is satisfied in the same frequency range for each fixed value of $s, \psi_{i}, \psi_{f}$, where $\psi_{i, f}$ is the angle between vectors $k_{i}$ and $p_{i, f}$, and the parameters of 'electronic medium' (i.e., the coefficients $A$ and $B$ ) will now depend on the intensity of the initial radiation fields.

Interaction of electrons with the intense radiation: 'non-diagonal' interaction. We now turn to the case of 'non-diagonal' interaction. Let $N_{i}$ - electrons carry out the process of pumping between waves $m_{s}$ (where $m_{s}=j_{1}, \ldots, j_{s}$, thus $\sum_{m_{s}}^{\infty} \equiv \sum_{j_{1}, \ldots, j_{s}}^{\infty}$ ). Then it is easy to obtain results for this 
A new look at some aspects of geometry, particle physics, inertia, radiation and cosmology

case from the final expressions of the already considered problem of 'diagonal' interaction after making appropriate replacements $k_{s}^{*} \rightarrow k_{m_{s}}^{*}, W_{s}\left(I_{i}\right) \rightarrow W_{m_{s}}\left(I_{m_{s}}\right)$, etc. But for the probability of the process we no longer have an explicit expression $W_{m_{s}}\left(I_{m_{s}} i\right)$, since the calculation of this quantity is associated with enormous difficulties. However, the complexity of the situation is to some extent compensated by the fact that the off-diagonal interaction involves only a tiny fraction of electrons, since the required conditions for electrons to be in the corresponding physical cones of formation pumping processes between different waves are very tough. For example, even in the simplest case of two $m_{s}=2$ oncoming waves, the physical cone of the formation of the pumping process is already delta-shaped, and meets the condition $1-\frac{v \cos \theta}{c} \frac{\nu_{1}+\nu_{2}}{\nu_{1}-\nu_{2}}=0$, where angle between vectors is the velocity of the electron, $\theta$ is the angle between this velocity and $e_{1}=-e_{2}, \nu_{1}, e_{1}$ and $\nu_{2}, e_{2}$ are the frequencies and the directional unit vectors of these waves. This means that the cone for cases $m_{s}>2$ will be even more narrow and, thus, harder to be satisfied.

\subsection{Transition coefficients for annihilation and creation of electron-positron pairs}

The above formalism of the probability coefficients transitions can be easily extended to the $t$ channel of the photon-electron interaction, namely to processes of annihilation and creation of electronpositron pairs. Since the Feynman diagram for these processes is topologically identical to the corresponding diagram of the $s$-channel of the photon-electron interaction, then the probability coefficients for the $t$-channel of the photon-electron interaction can be obtained directly from those found above (in item 1) by performing simple replacements. For example, for the annihilation process we have: $i \rightarrow-, E_{i} \rightarrow E_{-}, \nu_{i} \rightarrow-\nu_{1}, I_{i} \rightarrow I_{1}$, and $f \rightarrow+, E_{f} \rightarrow-E_{+}, \nu_{f} \rightarrow \nu_{2}, I_{f} \rightarrow I_{2}$, where $(-)$ and $(+)$ indicate the electronic and positron states. Whereas, depending on the specific problem, for the probability $W$ of the process one should take the probability of the process of annihilation or pairing. The interested reader is invited to consult the original papers (Ter-Kazarian, 1984c,e) for a complete set of derived explicit forms of Einstein's coefficients and the relations between them.

\section{The theory of Multiphoton Comptonization}

By means of the concept of 'effective photons', the integral kinetic equation was derived that describes the time evolution of the distribution function of quanta of non-equilibrium intense radiation for their multiphoton Compton scattering on Maxwellian nonrelativistic electrons (Ter-Kazarian, 1984a,b,d, 1987, 1989a,b). At first glance, it seems that the problem can hardly be reduced to the Fokker-Planck approximation, since the efficiency of electron acceleration in the field of an intense wave is large. Whereas, the thermal energy of electrons is much less than the energy acquired in the field of the intense wave. Therefore, consideration of issues of heat balance, etc. seems useless in this case. Moreover, one more difficulty arises regarding the distribution function of such electrons, which is essentially different from Maxwell's distribution function. To resolve these difficulties, the fact was used that the electron in both the initial and final states is in the field of initial radiation. Therefore, in multiphoton scattering, the process of transferring the energy of low-frequency photons to the short-wavelength part of the spectrum prevails. While the elementary act of stimulated multiphoton scattering by an electron with an effective four-momentum is replaced by another, completely equivalent to it, scattering of an 'effective photon' by a free electron, in a nonlinear mode, when the parameters of the electronic medium depend on the intensity of the initial radiation field. In the framework of this approach, the initial physical conditions of the problem are already imposed on the free states of electrons. The resulting Fokker-Planck approximation becomes valid when deriving an 'intermediate' kinetic equation for the distribution of 'effective photons'. Subsequently, the transition to the kinetic equation for the distribution of ordinary photons is made. The rearrangement of spectrum in case of a wide, in comparison with the Doppler profile, emission spectrum $\delta \gg \triangle \omega_{D s}^{*}=\omega_{s}^{*} \sqrt{2 k_{B} T_{e} / m_{e} c^{2}}$ is described by means of this differential equation. While the particular problem of the relaxation of the nonequilibrium isotopic radiation interacting with nondegenerate nonrelativistic electrons via the multiphoton Compton scattering is studied. Whereas, it is shown that the kinetic equation satisfies Boltzman's $H$-theorem for the coupled electron-photon system. The equations of heating and cooling of electron gas are derived. In (Ter-Kazarian, 1987, 1989c), we 
A new look at some aspects of geometry, particle physics, inertia, radiation and cosmology

study this problem in detail, and make estimate of various physical characteristics of compact objects of superhigh luminosity, as well as the efficiency of comptonization as an energy exchange process compared with the bremsstrahlung. In (Ter-Kazarian, 1989a), it was shown that the interpretation of the observational characteristics of a certain class of astrophysical objects, such as BLL B211308 +32 , OJ 287, NP 0532 pulsar and radio pulsars, the mechanism of multiphoton stimulated Compton scattering by electrons plays an important role. However, under real physical conditions occurring in the compact objects of high luminosity, the radiation is concentrated in the high frequency range $\delta \gg \triangle \omega_{D} \leq \omega_{D s}^{*}$ and solid angle $\Omega \ll 1$. Since scattering occurs only within the line, then due to the narrowness of the spectrum, the number of electrons involved in the process decreases and, consequently, the rate of the process decreases in comparison with a wide spectrum of radiation with the same brightness temperature. That is, with narrowing of the spectrum, both the transfer of radiation energy to electrons and the multiphoton induced pressure decrease. In (Ter-Kazarian, 1989b), we have continued the study of the process of relaxation of intense radiation on Maxwellian electrons in the general case of any spectral widths and any angular aperture of the radiation beam.

\section{Unique definition of relative velocity of luminous source}

For test particles and observers there is no unique way to compare four-vectors of the velocities at widely separated space-time events in a curved Riemannian space-time, because general relativity (GR) provides no a priori definition of relative velocity. This inability to compare vectors at different points was the fundamental feature of a curved space-time. Keeping in mind aforesaid, below we restrict our analysis to seeking solution for particular case when a test particle is being a luminous object. In this case, the problem of a definition of relative velocity can be significantly simplified because of available spectral shift measured by observer. The hope appears that a relative velocity of luminous source as measured along the observer's line-of-sight (speed) can be defined in unique way straightforwardly from kinematic spectral shift rule, which holds on a generic pseudo-Riemannian manifold (Synge, 1960). We extend those geometrical ideas developed by Synge, to build a series of infinitesimally displaced shifts and then sum over them in order to achieve an unique definition of the so-called kinetic relative velocity of luminous source, without subjecting it to a parallel transport, as measured along the observer's line-of-sight in a generic pseudo-Riemannian space-time. This provides a new perspective to solve startling difficulties of superluminal 'proper' recession velocities, which the conventional scenario of expanding universe of standard cosmological model presents. In some instances (in earlier epochs), the distant astronomical objects are observed to exhibit redshifts in excess of unity, and only a consistent theory could tackle the key problems of a dynamics of such objects.

\subsection{The relative velocity of luminous source as measured along the observer's line-of-sight in a generic pseudo-Riemannian space-time}

The principle foundation of our setup comprises the following steps. Let $(o)$ and $(s)$ be two world lines respectively of observer $O$ and source $S$ in the pseudo-Riemannian space-time. Suppose the passage of light signals from $S$ to $O$ is described by a single infinity of null geodesics $\Gamma(v)$ connecting their respective world lines. To clarify the issues further, it should help a few noteworthy points of Fig. 2. The $S_{(1)}$ and $S_{(2)}$ are two neighboring world points on $(s)$. The parametric values for these geodesics are $v, v+\Delta v$, respectively, where $v=$ const and $\Delta v$ is infinitesimally small. Accordingly, the world line $(s)$ is mapped pointwise on the $(o)$ by a set of null geodesics $\Gamma(v)$. That is, a set of null geodesics are joining $(s)$ to $(o)$, each representing the history of a wave crest. The totality of these null geodesics forms a 2-space with equation $x^{\mu}=x^{\mu}(u, v)$, which is determined once $(s)$ and $(o)$ are given. The $u$ denote the affine parameter on each of these geodesics running between fixed end-values $u=0$ on $(s)$ and $u=1$ on $(o)$. The $O_{(1)}$ and $\left.O_{(2}\right)$ are corresponding world points on $(o)$, where the null geodesics from $S_{(1)}$ and $S_{(2)}$ meet it. Also we will denote by $\tau_{O}$ and $\tau_{S}$ the proper times of the observer and the source, respectively, and $\triangle \tau_{O}$ and $\triangle \tau_{S}$ are the elements of proper time corresponding to the segments (the clock measures of) $O_{(1)} O_{(2)}$ and $S_{(1)} S_{(2)}$. Imagine now a dense family of adjacent observers $O_{j}$ $(j=1, \ldots, n-1)$ with the world lines $\left(o_{j}\right)$ populated between the two world lines $(o)$ and $(s)$. Each 


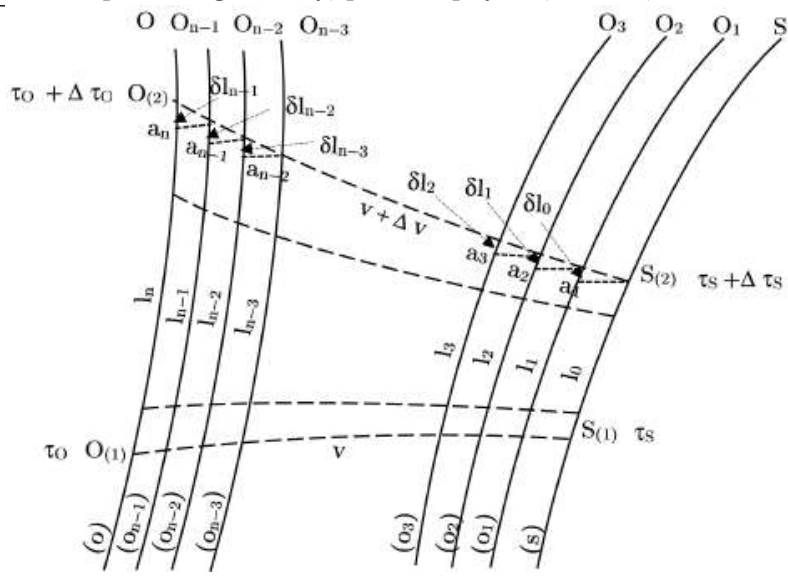

Figure 2. The infinitesimal spectral shifts as measured locally by emitter and adjacent receivers in a generic pseudo-Riemannian space-time. The $(o)$ and $(s)$ are two world lines respectively of observer $O$ and source $S$. A dense family of adjacent observers $O_{j}(j=1, \ldots, n-1)$ with the world lines $\left(o_{j}\right)$ populated between the two world lines $(o)$ and $(s)$. A set of null geodesics (the dotted lines) is mapping $(s)$ on $(o)$, each representing the history of a wave crest. Each line segment $l_{i-1}$ of proper space scale factor (at the affine parameter $u_{i-1}$ ) is identically mapped on the line segment $\left(l_{i}-\delta l_{i-1}\right)$ of proper space scale factor (at infinitesimally close affine parameter $u_{i}$ ), such that $l_{i-1} \equiv\left(l_{i}-\delta l_{i-1}\right.$ ), where $\delta l_{i}$ denotes infinitesimal segment $a_{i} O_{i(2)}$.

observer $O_{j}$ measures the frequency of light rays emitted by the source $S$ as it goes by. The $O_{j(1)}$ and $O_{j(2)}$ are two neighboring world points on $\left(o_{j}\right)$ where the null geodesics from $S_{(1)}$ and $S_{(2)}$ meet it. The $u_{j}$ denote the values of affine parameter on each of the null geodesics chosen at equal infinitesimally small $\delta u_{i}$, so that $u=u_{j}$ on $\left(o_{j}\right)$. The $\tau_{O_{j}}$ denotes the proper times of the adjacent observers, i.e. $\Delta \tau_{O_{j}}$ are the elements of proper time corresponding to the segment $O_{j(1)} O_{j(2)}$. Here and throughout we use the proper space scale factor $l_{i}(i=0,1,2, \ldots, n)$ which encapsulates the beginning and evolution of the elements of proper time $\Delta \tau_{S}$ of source, namely $l_{0}=c \Delta \tau_{S}, l_{1}=c \Delta \tau_{O_{(1)}}, \ldots, l_{n-1}=c \Delta \tau_{O_{n-1}}$, $l_{n}=c \Delta \tau_{O}$. Each line segment $l_{i-1}$ of proper space scale factor (at the affine parameter $u_{i-1}$ ) is identically mapped on the line segment $\left(l_{i}-\delta l_{i-1}\right)$ of proper space scale factor (at infinitesimally close affine parameter $\left.u_{i}\right)$, such that $l_{i-1} \equiv\left(l_{i}-\delta l_{i-1}\right)$, where $\delta l_{i}$ denotes infinitesimal segment $a_{i} O_{i(2)}$. If there are $N$ wave crests of the light, the wavelength of light $\lambda_{O_{i}}$ at the observers $O_{i}(i=1, \ldots, n$, where $\left.O_{n} \equiv O\right)$, who measures the wavelength of light ray as it goes by, satisfies the following condition: $N=l_{n} / \lambda_{n}=l_{n-1} / \lambda_{n-1}=\cdots=l_{1} / \lambda_{1}=l_{0} / \lambda_{0}$, where $\lambda_{i}\left(\equiv \lambda_{O_{i}}\right)$. The spectral shift, $z_{i}$, then reads $z_{i} \equiv \lambda_{i} / \lambda_{S}-1=l_{i} / l_{0}-1=\Delta \tau_{O_{i}} / \Delta \tau_{S}-1$. The spectral shift $z_{i}$, in general, can be evaluated straightforwardly in terms of the world function $\Omega\left(S O_{i}\right)$ for two points $S\left(x^{\prime}\right)$ and $O_{i}\left(x_{(i)}\right)(i=1, \ldots, n)$ through an integral defined along the geodesic $\Gamma_{S O_{i}}(v)$ joining them (Synge, 1960), taken along any one of the curves $v=$ const. The world function $\Omega\left(S O_{i}\right)$ can be defined for any of the geodesics in the family linking points on $\left(o_{i}\right)$ and $(s): \Omega\left(S O_{i}\right)=\Omega\left(x^{\prime} x_{(i)}\right) \equiv \Omega_{i}(v)=(1 / 2)\left(u_{O i}-u_{S}\right) \int_{u_{S}}^{u_{O i}} g_{\mu \nu} U^{\mu} U^{\nu} d u$, taken along $\Gamma_{S O_{i}}(v)$ with $U^{\mu}=d x_{(i)}^{\mu} / d u$, has a value independent of the particular affine parameter chosen. The holonomic metric $g=g_{\mu \nu} \vartheta \mu \otimes \vartheta^{\nu}=g\left(e_{\mu}, e_{\nu}\right) \vartheta^{\mu} \otimes \vartheta^{\nu}$, is defined in the Riemannian spacetime, with the components, $g_{\mu \nu}=g\left(e_{\mu}, e_{\nu}\right)(\mu=0,1,2,3)$ in the dual holonomic base $\left\{\vartheta^{\mu} \equiv d x^{\mu}\right\}$. For null geodesics $\Gamma_{S_{(1)}} O_{i(1)}(v)$ and $\Gamma_{S_{(2)}} O_{i(2)}(v+\Delta v)$, in particular, the world functions $\Omega_{(i)}(v)$ does not change in the interval $v$ and $v+\Delta v$, therefore $\left.\frac{\partial \Omega_{(i)}(v)}{\partial x^{\mu}} \frac{d x^{\mu}}{d v}\right|_{O_{i}}+\left.\frac{\partial \Omega_{(i)}(v)}{\partial x^{\mu}} \frac{d x^{\mu}}{d v}\right|_{S}=0$, which yields $1+z_{i}=$ $l_{i} / l_{0}=p_{\mu(S)} V_{(S)}^{\mu} / p_{\mu(i)} V_{(i)}^{\mu}$, where $V_{(i)}^{\mu}=d x^{\mu} /\left.d \tau_{O_{i}}\right|_{O_{i(1)}}$ and $V_{(S)}^{\mu}=d x^{\mu} /\left.d \tau_{S}\right|_{S_{(1)}}$ are the respective four-velocity vectors of observer $O_{i}$ and source $S$ (or world lines $\left(o_{i}\right)$ and $(s)$ ) at points $O_{i(1)}$ and $S_{(1)}$, $p_{(i)}^{\mu}=d x_{(i)}^{\mu} / d u_{i}$ and $p_{(S)}^{\mu}=d x^{\prime} \mu / d u_{0}$ are respective four-momenta of light ray (tangent to null geodesic) at the end points. For $i=n$, it becomes a well-known generalization of the overall spectral shift rule in a Riemannian space-time (Synge, 1960). Let us subject the vector $V_{(S)}^{\mu}$ to parallel transport along the null geodesic $\Gamma_{S_{(1)} O_{(1)}}(v)$ to the observer. This yields at $O_{(1)}$ the vector $\beta_{(S 1)}^{\mu}=\mathrm{g}^{\mu \nu^{\prime}}\left(O_{(1)}, S_{(1)}\right) V_{(S 1)}^{\nu^{\prime}}$, 
where the two point tensor $\mathrm{g}^{\mu \nu^{\prime}}\left(O_{(1)}, S_{(1)}\right)$ is the parallel propagator. The latter is determined only by the points $S_{(1)}$ and $O_{(1)}$. At $S_{(1)} \rightarrow O_{(1)}$, we have the coincidence limit $\left[\mathrm{g}^{\mu \nu^{\prime}}\right]\left(O_{(1)}\right)=g^{\mu \nu}\left(O_{(1)}\right)$. Then we obtain a relativistically invariant form of global Doppler shift (Synge, 1960): $z=1-[(1+$ $\left.\left.\beta_{\left(O_{(1)}\right)}^{2}\right)^{\frac{1}{2}}+\beta_{R\left(O_{(1)}\right)}\right]^{-1}$, where $c \beta_{(S 1)}^{\mu}=v_{(S 1)}^{\mu}, c \beta_{\left(O_{(1)}\right)}=v_{\left(O_{(1)}\right)}, c \beta_{R\left(O_{(1)}\right)}=v_{R\left(O_{(1)}\right)}$, and $v_{\left(O_{(1)}\right)}^{2}=$ $v_{(\alpha)\left(O_{(1)}\right)} v_{\left(O_{(1)}\right)}^{(\alpha)}, \quad v_{(\alpha)\left(O_{(1)}\right)}=v_{\mu(S 1)} \xi_{(\alpha)\left(O_{(1)}\right)}^{\mu}, \quad v_{R\left(O_{(1)}\right)}=v_{\mu(S 1))} r_{\left(O_{(1)}\right)}^{\mu}=v_{(\alpha)\left(O_{(1)}\right)} v_{\left(O_{(1)}\right)}^{(\alpha)}$. Reviewing notations the three-velocity of $(s)$ relative $(o)$ are defined by the tree invariant components $v_{(\alpha)\left(O_{(1)}\right)}$, $v_{(S 1)}$ is the relative speed, $v_{R\left(O_{(1)}\right)}$ is the speed of recession of $(s)$. Whereas $\xi_{(\alpha)\left(O_{(1)}\right)}^{\mu}$ is the frame of reference on world-line $(o)$ with $\xi_{0\left(O_{(1)}\right)}^{\mu}=V_{\left(O_{(1)}\right)}^{\mu}$, the unit vector $r^{\mu\left(O_{(1)}\right)}$ at $O_{(1)}$ is orthogonal to world-line $(o)\left(r_{\mu\left(O_{(1)}\right)} V_{\left(O_{(1)}\right)}^{\mu}=0\right)$ and lying in the 2-element which contains the tangent at $O_{(1)}$ to (o) and $S_{(1)} O_{(1)}$.

In studying further a set of null geodesics $\Gamma(v)$ with equations $x^{\mu}\left(u_{i}, v\right)$ (where $v=$ const), we may deal with the deviation vector $\eta_{(i)}^{\mu}$ drawn from $O_{i(1)} S_{(1)}$ to $O_{i(2)} S_{(2)}$, and that we have along null geodesic $\eta_{\mu(i)} \partial x^{\mu} / \partial u_{i}=$ const. Then the infinitesimal 'relative' spectral shift $\delta z_{i}$ between the observers $O_{i+1}$ and $O_{i}$ will be $\delta z_{i}=p_{\mu(i)} V_{(i)}^{\mu} / p_{\mu(i+1)} V_{(i+1)}^{\mu}-1=\Omega_{\mu(i)} V_{(i)}^{\mu} / \Omega_{\mu(i+1)} V_{(i+1)}^{\mu}-1$, where $E_{i}=p_{\mu(i)} V_{(i)}^{\mu}$ is the energy of light ray relative to an observer $O_{i}$, and $\Omega_{\mu(i)}=\left(u_{O i}-u_{S}\right) U_{\mu}$. For definiteness, let consider case of $l_{n}>l_{0}$ (being red-shift, Fig. 1). In similar way, of course, we may treat a negative case of $l_{n}<l_{0}$ (being blue-shift), but it goes without saying that in this case a source is moving towards the observer. In first case, the observers at the points $O_{i(2)}(i=1, \ldots, n-1)$ should observe the monotonic increments of 'relative' spectral shifts $\left(\delta z_{1}, \delta z_{2}, \delta z_{3}, \ldots, \delta z_{n-1}\right)$ when light ray passes across the infinitesimal distances $\left(O_{1(2)}, S_{(2)}\right),\left(O_{2(2)}, O_{1(2)}\right), \ldots,\left(O_{n(2)}, O_{(n-1)(2)}\right)$. Thus, the wavelength of light emitted at $S_{(2)}$ is stretched out observed at the points $O_{i(2)}$. While weak, such effects considered cumulatively over a great number of successive increments of 'relative' spectral shifts could become significant. The resulting spectral shift is the accumulation of a series of infinitesimal shifts as the light ray passes from luminous source to adjacent observers along the path of light ray. This interpretation holds rigorously even for large spectral shifts of order one or more. If this view would prove to be true, then it would lead to the chain rule for the wavelengths $\frac{\lambda_{O}(n 2)}{\lambda_{0}} \equiv \frac{\lambda_{n}}{\lambda_{1}}=\frac{\lambda_{n}}{\lambda_{n-1}} \cdot \frac{\lambda_{n-1}}{\lambda_{n-2}} \cdots \frac{\lambda_{2}}{\lambda_{1}} \cdot \frac{\lambda_{1}}{\lambda_{0}}=$ $\prod_{i=1}^{n-1}\left(1+\delta z_{i}\right)=\prod_{i=1}^{n-1} p_{\mu(i)} V_{(i)}^{\mu} / p_{\mu(i+1)} V_{(i+1)}^{\mu}=\prod_{i=1}^{n-1} \Omega_{\mu(i)} V_{(i)}^{\mu} / \Omega_{\mu(i+1)} V_{(i+1)}^{\mu}$, where $\lambda_{0} \equiv \lambda_{S_{(2)}}$. With no loss of generality, we may of course apply it all the way to $n \rightarrow \infty$. Let us view the increment of the proper space scale factor, $l_{i}=l\left(u_{i}\right)$, over the affine parameters $u_{i} \quad(i=1,2, \ldots, n)$ as follows: $l_{i}=l_{0}+i \varepsilon$, where $\varepsilon$ can be made arbitrarily small by increasing $n$. In the limit $n \rightarrow \infty$, all the respective adjacent observers are arbitrarily close to each other, so that $\delta z_{i}=\delta l_{i} / l_{i} \simeq \varepsilon / l_{0} \rightarrow 0$. This allows us to write the following relation for the infinitesimal 'relative' redshifts: $\left(\delta z_{n-1}=\delta z_{n-2}=\cdots=\delta z_{1}=\right.$ $\left.\varepsilon / l_{0}\right)_{n \rightarrow \infty}=\lim _{n \rightarrow \infty} \delta z_{(n-1)}^{(a)} \equiv \lim _{n \rightarrow \infty}\left(\frac{1}{n-1} \sum_{i=1}^{n-1} \delta z_{i}\right)$, provided, $\delta z_{(n-1)}^{(a)}$ is the average infinitesimal increment of spectral shift. Hence, $1+z=\lim _{n \rightarrow \infty}\left(1+\delta z_{(n-1)}^{(a)}\right)^{n-1}$, where $\Omega_{\mu(O)}=\left(u_{O}-u_{S}\right) U_{\mu(O)}$ and $\Omega_{\mu(S)}=-\left(u_{O}-u_{S}\right) U_{\mu(S)}$. It should be emphasized that this general equation is the result of a series of infinitesimal stretching of the proper space scale factor in Riemannian space-time, whereas the path of a luminous source appears nowhere, thus this equation does not relate to the special choice of transport path. Then, the transformation of this equation by means of a particular definition of infinitesimal relative velocity of luminous source to observer in Riemannian space-time cannot be accepted in general, because in such approach there is no relative velocity without prior choice of transport paths. The statement attributing frequency shifts to the resulting relative velocity cannot be accepted either. To overcome the ambiguity of parallel transport of four-vectors in curved space-time, in what follows we advocate with alternative proposal. The infinitesimal increments of 'relative' spectral shifts $\left(\delta z_{1}, \delta z_{2}, \delta z_{3}, \ldots, \delta z_{n-1}\right)$ can be derived from Doppler effect between adjacent emitter and absorber in relative motion measured in the respective tangent local inertial rest frames at infinitesimally separated space-time points. To obtain some feeling about this statement, below we give more detailed explanation. Imagine a family of adjacent observers $\left(O_{a_{i}}\left(u_{i}\right)\right)$ situated at the points $a_{i}(i=1, \ldots, n)$ on the world lines $\left(o_{i}\right)$ at infinitesimal distances from the observers $\left(O_{i(2)}\right)$, who measure the wavelength of radiation in relative motion which cause a series of infinitesimal stretching $\left(\delta l_{0}, \ldots, \delta l_{n-1}\right)$ of the proper space scale factor. Since each line segment $l_{i-1}$ of proper space scale factor 
(at the affine parameter $\left.u_{i-1}\right)$ is identically mapped on the line segment $\left(l_{i}-\delta l_{i-1}\right)$ (where $\delta l_{i}$ denotes infinitesimal segment $a_{i} O_{i(2)}$ ) of proper space scale factor (at the affine parameter $u_{i}=u_{i-1}+\delta u_{i-1}$ ), the relative speed $v_{O_{i(2)} O_{a_{i}}}\left(u_{i}\right)$ of observer $\left(O_{i(2)}\left(u_{i}\right)\right)$ to adjacent observer $\left(O_{a_{i}}\right)\left(u_{i}\right)$ should be the same as it is relative to observer $\left(O_{(i-1)(2)}\left(u_{i-1}\right)\right)$, that is $v_{O_{i(2)} O_{(i-1)}}\left(u_{i-1}\right) \equiv v_{O_{i(2)}} O_{a_{i}}\left(u_{i}\right)$. Continuing along this line, we may commit ourselves in the series of 'relative' spectral shifts, equivalently, a certain substitution of increments of relative speeds. Taking into account that the infinitesimal speeds of source $(S)$ relative to observers $\left(O_{i(2)}\right)$ arise at a series of infinitesimal stretching of the proper space scale factor $\delta l_{i}(i=1,2, \ldots, n)$ as it is seen from the Fig. 1 , we may fill out the whole pattern of monotonic increments of 'relative' spectral shifts $\left(\delta z_{1}, \delta z_{2}, \delta z_{3}, \ldots, \delta z_{n-1}\right)$ by, equivalently, replacing the respective pairs $\left(O_{1(2)}, S_{(2)}\right),\left(O_{2(2)}, O_{1(2)}\right), \ldots,\left(O_{n(2)}, O_{(n-1)(2)}\right)$ with new ones $\left(O_{1(2)}, O_{a_{1}}\right),\left(O_{2(2)}, O_{a_{2}}\right), \ldots,\left(O_{n(2)}, O_{a_{n}}\right)$, which attribute to the successive increments of relative speeds $v_{O_{1(2)} S}\left(u_{1}\right), \ldots, v_{O_{n(2)} O_{(n-1)(2)}}\left(u_{n}\right)$ of the source $(S)$ away from an observer $\left(O_{n(2)}\right)$ in the rest frame of $\left(O_{n(2)}\right)$, viewed over all the values $(i=1, \ldots, n)$. This framework furnishes justification for the concept of relative speed $c \beta_{n} \equiv v_{O_{n(2)} S_{(2)}}$, to be now referred to as the kinetic relative velocity, of the source $(S)$ to observer $\left(O_{n(2)}\right)$ along the line of sight. At the limit $n \rightarrow \infty$, the relative infinitesimal speeds tends to zero, $v_{O_{i(2)} O_{a_{i}}}\left(u_{i}\right)=c \delta \beta_{i}=c \delta z_{i}=c \delta l_{i} / l_{i} \simeq c \varepsilon / l_{0} \rightarrow 0$, such that $v_{O_{i(2)} O_{a_{i}}}\left(u_{i}\right)=c \delta \beta_{i}=c \delta z_{i}=c \delta l_{i} / l_{i} \simeq c \varepsilon / l_{0} \rightarrow 0$, such that $\lim _{n \rightarrow \infty} \delta \beta_{n-1}=\delta \beta^{(a)}(\equiv$ $\left.\lim _{n \rightarrow \infty}(n-1)^{-1} \sum_{i=1}^{n-1} \delta \beta_{i}\right)=\lim _{n \rightarrow \infty} n^{-1} \beta_{n}$.

Remark: Although we are free to deal with any infinitesimal 'relative' spectral shift $\delta z_{i}$ for the pair $\left(O_{i(2)}\right)$ and $\left(O_{a_{i}}\right)$, in local tangent inertial rest frame of an adjacent observer $\left(O_{a_{i}}\right)$, where we may approximate away the curvature of space in the infinitesimally small neighborhood, nevertheless, the infinitesimal relative velocities generally arise in RW space-time at a series of infinitesimal stretching of the proper space scale factor as alluded to above, so that the SR law of composition of velocities cannot be implemented globally along non-null geodesic because these velocities are velocities at the different events, which should be in a different physical frames, and cannot be added together.

Facilitating further the calculations of recession velocity $\left(\beta_{n}\right)$ in quest, therefore, we may address the pair of observers at points $O_{(n) 2}$ and $a_{n}$. Suppose $V_{O_{n(2)}}^{\mu}$ and $V_{O_{a_{n}}}^{\mu}$ be the unit tangent four-velocity vectors of observers $\left(O_{n(2)}\right)$ and $\left(O_{a_{n}}\right)$ to the respective world-lines in a general Riemannian space-time, thus in their respective rest frame we have $V_{O_{n(2)}}^{0}=1$ and $V_{O_{a_{n}}}^{0}=1$, as the only nonzero components of velocity. For comparing the vectors $V_{O_{n(2)}}^{\mu}$ and $V_{O_{a_{n}}}^{\mu}$ at different events, it is necessary to seek a useful definition of the relative velocity by bringing both vectors to a common event by subjecting one of them to parallel transport. Since all the paths between infinitesimally separated space-time points $O_{(n) 2}$ and $a_{n}$ are coincident at $n \rightarrow \infty$, for comparing these velocities there is no need to worry about specific choice of the path of parallel transport of four-vector. Therefore, we are free to subject further the unit tangent four-velocity vector $V_{O_{n(2)}}^{\mu}$ to parallel transport along the null geodesic $\Gamma_{O_{n(2) a_{n}}}$ to the point $a_{n}$. Thereby, the ray passes an observer $O_{a_{n}}\left(u_{n}\right)\left(\equiv O_{(n-1)(2)}\left(u_{n-1}\right)\right)$ with the proper space scale factor $l_{n-1}$ who measures the wavelength to be $\lambda_{n-1}$. The ray passes next observer $O_{n(2)}\left(u_{n}\right)$ with the proper space scale factor $l_{n}=l_{n-1}+\delta l_{n-1}$. The ray's wavelength measured by observer $O_{n(2)}\left(u_{n}\right)$ is increased by $\delta \lambda_{n-1}=\lambda_{n}-\lambda_{n-1}$ leading to infinitesimal 'relative' spectral shift $\delta z_{n-1}$. A parallel transport yields at $O_{a_{n}}$ the vector $\beta_{\mu\left(O_{a_{n}}\right)}=\mathrm{g}_{\mu \nu^{\prime}}\left(O_{a_{n}}, O_{(n) 2}\right) V_{O_{(n) 2}}^{\nu^{\prime}}$, where the two point tensor $\mathrm{g}_{\mu \nu^{\prime}}\left(O_{a_{n}}, O_{(n) 2}\right)$ is the parallel propagator as before, which is now determined by the points $O_{(n) 2}$ and $O_{a_{n}}$. At $O_{(n) 2} \rightarrow O_{a_{n}}$, we have the coincidence limit $\left[\mathrm{g}_{\mu \nu}\right]\left(O_{a_{n}}\right)=g_{\mu \nu}\left(O_{a_{n}}\right)$. As we have at point $O_{a_{n}}$ two velocities $V_{O_{a_{n}}}^{\mu}$ and $\beta_{\left(O_{a_{n}}\right)}^{\mu}=g^{\mu \nu} \beta_{\nu\left(O_{a_{n}}\right)}$, we may associate Doppler shift $\delta z_{n-1}$ to four-velocity $\beta_{\left(O_{a_{n}}\right)}^{\mu}$ of observer $O_{n}$ observed by an observer $O_{a_{n}}$ with four-velocity $V_{O_{a_{n}}}^{\mu}$ as measured by the latter. Consequently, the three-velocity of an observer $\left(O_{n(2)}\right)$ relative to observer at $\left(O_{a_{n}}\right)$ is $v_{(\alpha)\left(O_{a_{n}}\right)}$, the relative speed is $v_{\left(O_{a_{n}}\right)}$, and $v_{R\left(O_{a_{n}}\right)}$ is the speed of recession of $\left(o_{n}\right)$. In the local inertial rest frame $\xi_{(\alpha)\left(O_{a_{n}}\right)}^{\mu}$ of an observer $\left(O_{a_{n}}\right)$, the velocity vector $\beta_{O_{a_{n}}}^{\mu}$ takes the form $\left(\gamma, \gamma \delta \beta_{\left(O_{a_{n}}\right)}, 00\right)$, where an observer $\left(O_{n}(2)\right)$ is moving away from the observer $\left(O_{a_{n}}\right)$ with the relative infinitesimal three-velocity $\delta \beta_{\left(O_{a_{n}}\right)}$ (in units of the speed of light) in a direction making an angel $\theta_{\left(O_{a_{n}}\right)}$ with the outward direction of line of sight $\Gamma_{O_{n(2) O_{a_{n}}}}$ from $O_{(n) 2}$ to $O_{a_{n}}$, and $\gamma=\left(1-\delta \beta_{\left(O_{a_{n}}\right)}^{2}\right)^{-1 / 2}$. Hence, $\delta z_{n-1}=\delta \beta_{\left(O_{a_{n}}\right)} \cos \theta_{\left(O_{a_{n}}\right)}$. Thus, at $n \rightarrow \infty$, the wavelength measured by the observer $O_{n(2)}$ is increased by the first-order Doppler shift 


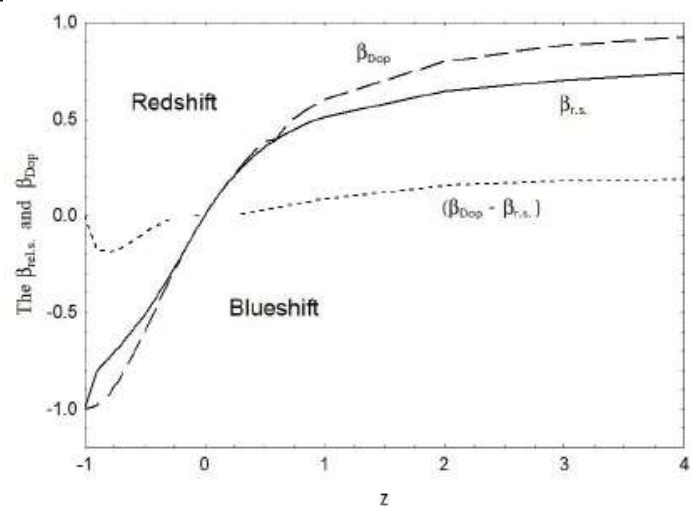

Figure 3 . The relative velocity along the line of sight $\left(\beta_{\text {r.s. }}\right)$ of luminous source $(S)$ with $-1 \leq z \leq 4$ to observer $(O)$, the global Doppler velocity $\left(\beta_{D o p}\right)$, and their difference (in units of the speed of light).

caused unambiguously by the infinitesimal relative speed $\delta \beta_{n-1}^{(r)} \equiv \delta \beta_{\left(O_{a_{n}}\right)} \cos \theta_{\left(O_{a_{n}}\right)}$ along the line of sight with end-points $O_{(n) 2}$ and $O_{a_{n}}: \delta z_{n-1}=\delta l_{n-1} / l_{n-1}=\delta \beta_{n-1}^{(r)}$. The SR law of composition of velocities along the line of sight can be implemented in the tangent inertial rest frame of an observer $O_{a_{n}}: \delta \beta_{n-1}^{(r)}=\left(\beta_{n}-\beta_{n-1}\right) /\left(1-\beta_{n} \beta_{n-1}\right) \simeq \delta \beta_{n-1} /\left(1-\beta_{n-1}^{2}\right)$, where $v_{n-1}=c \beta_{n-1}$ and $v_{n}=c \beta_{n}$ are, respectively, the three-velocities of observers $O_{a_{n}}$ and $O_{n}$ along the line of sight with end-points $O_{a_{n}}$ and $O_{(n) 2}$. Consequently, the relations presented above result in the straightforward kinematic relationship of the overall spectral shift, $z$, and the speed $\beta_{\text {r.s. }}$ (in units of the speed of light) of source $(S)$ relative to observer $\left(O \equiv O_{n}\right)$ in its rest frame along the line of sight in a general Riemannian space-time: $\left.1+z=p_{\mu S} V_{S}^{\mu} / p_{\mu O} V_{O}^{\mu}=\Omega_{\mu(S)} V_{(S)}^{\mu} / \Omega_{\mu(O)} V_{(O)}^{\mu}=\exp \left[\beta_{r . s .}\right) /\left(1-\beta_{r . s .}^{2}\right)\right]$, where the relative speed $\beta_{r . s .} \equiv \lim _{n \rightarrow \infty} \beta_{n}$ in quest is marked with subscript ( $)_{r . s .}$. The relative speed of luminous source is plotted on the Fig. 3 for redshifts $-1 \leq z \leq 4$.

\subsection{A global Doppler shift along the null geodesic}

Suppose the velocities of observers say $O_{i(2)}(i=1, \ldots, n-1)$, being in free fall, populated along the null geodesic $\Gamma_{S_{(2)} O_{(2)}}(v+\Delta v)$ of light ray (Fig. 2), vary smoothly along the line of sight with the infinitesimal increment of relative velocity $\delta \beta_{i}^{r}$. The $(i)$-th observer situated at the point $i(2)$ of intersection of the ray's trajectory $\Gamma_{S_{(2)} O_{(2)}}(v+\Delta v)$ with the world line $\left(o_{i}\right)$ at affine parameter $u_{i}$, and measures the frequency of light ray as it goes by. According to the equivalence principle, we may approximate away the curvature of space in the infinitesimally small neighborhood of two adjacent observers. We should emphasize that if we approximate an infinitesimally small neighborhood of a curved space as flat, the resulting errors are of order $\left(\delta l_{i} / l_{n}\right)^{2}$ in the metric. If we regard such errors as negligible, then we can legitimately approximate space-time as flat. The infinitesimal increment of spectral shift $\delta z_{i}$ is not approximated away in this limit because it is in that neighborhood of leading order $\left(\delta l_{i} / l_{i}\right)$. That is, approximating away the curvature of space in the infinitesimally small neighborhood does not mean approximating away the infinitesimal increment $\delta z_{i}$. Imagine a thin world tube around the null geodesic $\Gamma_{S_{(2)} O_{(2)}}(v+\Delta v)$ within which the space is flat to arbitrary precision. Each observer has a local reference frame in which SR can be taken to apply, and the observers are close enough together that each one $O_{(i+1)(2)}$ lies within the local frame of his neighbor $O_{i(2)}$. This implies the vacuum value of a velocity of light to be universal maximum attainable velocity of a material body found in this space. Such statement is true for any thin neighborhood around a null geodesic. Only in this particular case, the relative velocity of observers can be calculated by the SR law of composition of velocities globally along the path of light ray. We may apply this law to relate the velocity $\beta_{i+1}$ to the velocity $\beta_{i}$, measured in the $i$-th adjacent observer's rest frame. The end points of infinitesimal distance between the adjacent observers $O_{(i+1)(2)}$ and $O_{i(2)}$ will respectively be the points of intersection of the ray's trajectory with the world lines $o_{i+1}\left(u_{i+1}\right)$ and $\left(o_{i}\right)\left(u_{i}\right)$. This causes a series of infinitesimal increment of the proper space scale factor from initial value $l_{0}=\Delta \tau_{S}$ to the given value $l_{i}=\Delta \tau_{O_{i}}$, which in turn causes a series of infinitesimal increment of spectral shift 
$\delta z_{i}=\delta \lambda_{i} / \lambda_{i}=\delta l_{i} / l_{i}$. Within each local inertial frame, there are no gravitational effects, and hence the infinitesimal spectral shift from each observer to the next is a Doppler shift. Thus, at the limit $n \rightarrow \infty$, a resulting infinitesimal frequency shift $\delta z_{i}$, can be unambiguously equated to infinitesimal increment of a fractional SR Doppler shift $\delta \bar{z}_{i}$ from observer $O_{i(2)}$ to the next $O_{(i+1)(2)}$ caused by infinitesimal relative velocity $\delta \bar{\beta}_{i}^{r}:\left(\delta z_{i}=\delta l_{i} / l_{i}\right)_{n \rightarrow \infty}=\left(\delta \bar{z}_{i}=\delta \bar{\beta}_{i}^{r}=\left(\bar{\beta}_{i+1}-\bar{\beta}_{i}\right) /\left(1-\bar{\beta}_{i+1} \bar{\beta}_{i}\right) \simeq \delta \bar{\beta}_{i} /\left(1-\bar{\beta}_{i}^{2}\right)\right)_{n \rightarrow \infty}$, where by $(\overline{)}$ we denote the null-geodesic value, as different choice of geodesics yields different results for the motion of distant test particles relative to a particular observer. This relation can be transformed as follows: $\left(\delta z_{n-1}=\right)_{n \rightarrow \infty}=\left(\delta \beta_{n-1} /\left(1-\beta_{n}^{2}\right)\right)_{n \rightarrow \infty}=\left(\delta \bar{z}_{(n-1)}^{(a)}=\delta \bar{\beta}_{(n-1)}^{r(a)} \equiv(n-1)^{-1} \sum_{i=1}^{n-1} \delta \bar{\beta}_{i} /(1-\right.$ $\left.\left.\bar{\beta}_{i}^{2}\right)\right)_{n \rightarrow \infty}$, which, for sufficiently large but finite $n$, gives $\bar{\beta}_{n}=\left(e^{\varrho_{n}}-1\right) /\left(e^{\varrho_{n}}+1\right), \quad \varrho_{n} \equiv 2 \beta_{n} /\left(1-\beta_{n}^{2}\right)$. Hence the general solution is reduced to a global Doppler shift along the null geodesic $1+z=$ $\sqrt{1+\bar{\beta}_{r . s .} / 1-\bar{\beta}_{r . s .}}=p_{\mu(O 2)} V_{(S 2)}^{\mu} / p_{\mu(O 2)} V_{(O 2)}^{\mu}$, where $\bar{\beta}_{r e c}=\lim _{n \rightarrow \infty} \bar{\beta}_{n}, V_{(S 2)}^{\mu}$ and $V_{(O 2)}^{\mu}$ are the fourvelocity vectors, respectively, of the source $S_{(2)}$ and observer $O_{(2)}, p_{\mu(S 2)}$ and $p_{\mu(O 2)}$ are the tangent vectors to the typical null geodesics $\Gamma_{S_{(2)}} O_{(2)}(v)$ at their respective end points. This procedure, in fact, is equivalent to performing parallel transport of the source four-velocity in a general Riemannian spacetime along the null geodesic to the observer. Note that any null geodesic from a set of null geodesics mapped $(s)$ on $(o)$ can be treated in the similar way. In Minkowski space a parallel transport of vectors is trivial and mostly not mentioned at all. This allows us to apply globally the SR law of composition of velocities to relate the velocities $\bar{\beta}_{i+1}$ to the $\bar{\beta}_{i}$ of adjacent observers along the path of light ray, measured in the $i$-th adjacent observer's frame. Then, a global Doppler shift of light ray emitted by luminous source as it appears to observer at rest in flat Minkowski space can be derived by summing up the infinitesimal Doppler shifts caused by infinitesimal relative velocities of adjacent observers.

\section{The implications for the spatially homogeneous and isotropic Robertson-Walker space-time}

In the framework of RW-cosmological model, one assumes that the universe is populated with comoving observers (Ter-Kazarian, 2021a). In the homogeneous, isotropic universe comoving observers are in freefall, and obey Wayl's postulate: their all worldlines form a 3-bundle of non-intersecting geodesics orthogonal to a series of spacelike hypersurfaces, called comoving hypersurfaces. In case of expansion, all worldlines are intersecting only at one singular point. The clocks of comoving observers, therefor, can be synchronized once and for all. Let the proper time, $t$, of comoving observers be the temporal measure. Suppose $R(t)$ is the scale factor in expanding homogenous and isotropic universe. One considers in the so-called cosmological rest frame a light that travels from a galaxy to a distant observer, both of whom are at rest in comoving coordinates. As the universe expands, the wavelengths of light rays are stretched out in proportion to the distance $L(t)$ between co-moving points $\left(t>t_{1}\right)$, which in turn increase proportionally to $R(t)$. In this case, the role of proper space scale factor $l_{i}$ is now destined to the scale factor $R\left(t_{i}\right) \propto L\left(t_{i}\right)$. The general solution, of course, does straightforwardly yield the particular solution as a corollary for the case of expanding RW-space-time of standard cosmological model. However, it is instructive to substantiate this principle statement further by the reasoning recast in more physical terms of, alternative, so-called lookforward history of expanding homogenous and isotropic universe. As a guiding principle, therefore, we briefly recount some of the highlights behind of this approach. The Fig. 4 illustrates the lookforward history, where $L_{i} \equiv L\left(t_{i}\right)$ is the 'proper distance' between a galaxy $\left(A_{i}\right)$ and observer $\left(O_{i}\right)$, at given epoch $\left(t_{i}\right)$, while the increase of the $L_{i}$ is viewed over different epochs $(i=1,2, \ldots, n)$, with the infinitesimal time difference $\left(\left(t_{i}-t_{i-1}\right) \rightarrow 0, n \rightarrow \infty\right)$. To give more credit to this view, we go ahead with the lookforward history of the proper distance $L_{1} \equiv L\left(t_{1}\right)$ in the expanding universe, from initial epoch $\left(t_{1}\right)$ to the present epoch $(t)$. Thereby $t_{n} \equiv t$ and $L_{n} \equiv L(t)$. We assume that an observer $\left(O_{i}\right)$ in its rest frame of reference measures the frequency of light rays emitted by a galaxy $\left(A_{i}\right)$ viewed over different epochs $(1, \ldots, i)$ of expansion. Each proper distance $L_{A_{i-1} O_{i-1}}\left(t_{i-1}\right)$ (at epoch $t_{i-1}$ ) is identically mapped on the line segment $L_{a_{i} O_{i}}\left(t_{i}\right)$ of proper distance (at infinitesimally close epoch $t_{i}$ ), such that $L_{a_{i} O_{i}}\left(t_{i}\right) \equiv L_{A_{i-1} O_{i-1}}\left(t_{i-1}\right)$. Null geodesic of a light signal from a galaxy $\left(A_{1}\right)$ to the observers $O_{i}$ $\left(O_{n} \equiv O\right)$ is also plotted. The picture on Fig. 4, of course, wholly agrees with the Cosmological Principle. The requirement for homogeneity and isotropy is implemented by this principle in order to 


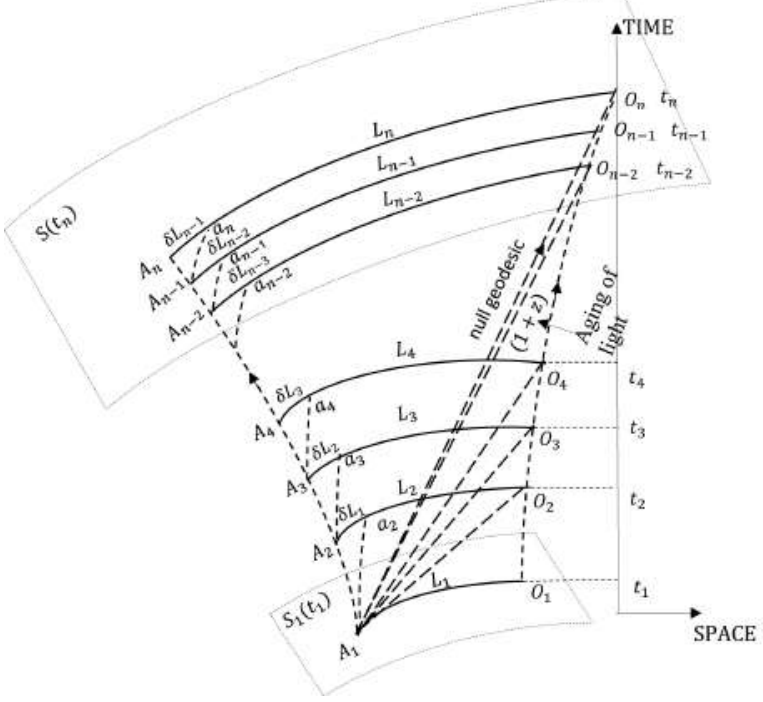

Figure 4. 'Lookforward' history of expanding homogenous and isotropic universe.

avoid a privileged observer. In accord to modern cosmology, the universe does not expand in space, but consists of expanding space. It does not say anything about the point of origin of the universe, either it does not mean that every pair of galaxy $\left(A_{i}\right)$ and observer $\left(O_{i}\right)$ is in any specially favoured or unfavoured position in the universe: the universe is isotropic about this pair, which moving apart as universe expands. Keeping in mind aforesaid, now let us explore the definition of Hubble's parameter to write $H=\frac{d}{d t} \log \left(\frac{R(t)}{R_{1}}\right)=\frac{d}{d t} \ln (1+z)=\frac{1}{1+z} \frac{d z}{d t}$, which, incorporating with the relation $d t / d t_{1}=1+z$, yields $d L_{1} / d t_{1}=\dot{L}-H L=0$. The net result is as the relation $\lambda_{A_{1}}=\lambda_{O_{(1)}}$ holds for the wavelengths.

Imagine a family of comoving adjacent observers situated at the points $a_{i}(i=2, \ldots, n)$ on the infinitesimal distances from the galaxies $\left(A_{i}\right)$, who measure the frequency of light rays emitted by $\left(A_{i}\right)$ as it goes by. Consequently, an observers should observe the successive increments of 'relative' redshifts, $\delta z_{1}, \delta z_{2}, \delta z_{3}, \ldots, \delta z_{n-1}$, of the light when passing across the infinitesimal distances $\left(A_{2}, a_{2}\right),\left(A_{3}, a_{3}\right), \ldots,\left(A_{n}, a_{n}\right)$. Thus, the wavelength of light emitted at $A_{i}$ is infinitesimally stretched out relative to the wavelength of light emitted at the adjacent point $a_{i}$. While weak, such effects considered cumulatively over a great number of successive increments of redshifts could become significant. The resulting redshift is the accumulation of a series of infinitesimal redshifts as the light passes from receding galaxy to adjacent observers along the line of sight. This interpretation holds rigorously even for large redshifts of order one or more. Continuing along this line, in what follows, the mathematical structure has much in common with those constructions of subsection 10.1. Here we shall forbear to write them out as they are explained so well. We finally achieve a general solution, which is now reduced to the straightforward kinematic relationship $1+z=R(t) / R\left(t_{1}\right)=\exp \left[\beta_{r e c} /\left(1-\beta_{r e c}^{2}\right)\right]$. A crux is the more rigorous viable concept of physical recession velocity $\left(\beta_{\text {rec }} \equiv \beta_{\text {rel.s. }}\right)$ of a comoving distant galaxy of redshift $z$, which crossed past light cone at time $t_{1}$, at point $\left(A_{1}\right)$ away from comoving observer $(O)$ at the present time $t$. This interpretation so achieved has physical significance as it agrees with a view that the light waves will be stretched by travelling through the expanding universe, and in the same time the kinetic recession velocity of a distant astronomical object is always subluminal even for large redshifts of order one or more. It, therefore, does not violate the fundamental physical principle of causality. This provides a new perspective to solve startling difficulties of superluminal 'proper' recession velocities, which the conventional scenario of expanding universe of standard cosmological model presents. In some instances (in earlier epochs), the distant astronomical objects are observed to exhibit redshifts in excess of unity, and only a consistent theory could tackle the key problems of a dynamics of such objects. Moreover, the general solution is reduced to global Doppler shift along the null geodesic, studied by Synge. If, and only if, for the distances at which the Hubble empirical linear 'redshift-distance' law $(c z=H L)$ is valid, the relationship between the physical recession velocity, $v_{r e c}$, and the expansion rate, $\dot{L}(=H L)$, reads $\beta_{\text {rec }}=\left[\sqrt{1+4 \ln ^{2}(1+\dot{L} / c)}-1\right] / 2 \ln (1+\dot{L} / c)$. 


\section{The physical outlook and concluding remarks}

The physical outlook and concluding remarks on suggested novel aspects of geometry and high energy physics, spacetime deformation induced inertia effects and intense radiation physics, are given in the following items in order to resume once again a whole physical picture.

- We show how the curvature and torsion, which are properties of a connection of geometry under consideration, will come into being? The theoretical significance resides in constructing the theory of the two-step spacetime deformation as a guiding principle. We construct the TSSD-versions of the theory of teleparallel gravity and the most important EC theory. It is remarkable that the equations of the standard EC theory, in which the equation defining torsion is the algebraic type and, in fact, no propagation of torsion is allowed, can be equivalently replaced by the set of modified Einstein-Cartan equations in which the torsion, in general, is a dynamical. The special physical constraint imposed upon the spacetime deformations yields the short-range propagating spin-spin interaction and the existence of torsion waves that may contribute a new special polarized effects in the neutron interferometry and gravitational waves experiments. We can observe the effect of the polarized rotation plane due to quantum interferometry, which would be an interesting topic not discussed in this paper. A detailed analysis and calculations on the more general MAG theory with dynamical torsion in context of TSSD formulation of post-Riemannian geometry will be presented in another paper to follow at a later date.

- In the same framework we shown that the equations of the standard MAG theory can be equivalently replaced by the set of modified MAG equations in which the torsion, in general, is dynamical. As an application we have to test the general TSSD-MAG framework in some limit, namely we have to put on Lagrange multipliers to recover the TSSD-versions of different (sub-)cases of Poincaré gauge theory $(\mathrm{PG})$, Einstein-Cartan (EC) theory, teleparallel gravity $\left(\mathrm{GR}_{\|}\right)$and general relativity (GR). Moreover, we shown that by imposing different appropriate physical constraints upon the spacetime deformations, in this framework we may reproduce the term in the well known Lagrangian of pseudoscalar-photon interaction theory, or terms in the Lagrangians of pseudoscalar theories by $\mathrm{Ni}$, or in modification of electrodynamics with an additional external constant vector coupling, as well as in case of intergrand for topological invariant, or in case of pseudoscalar-gluon coupling occurred in QCD in an effort to solve the strong CP problem.

- The new conceptual element in the extended phase space formulation is noteworthy. Extended canonical transformations allows to go from one extended phase space to another. This unifying feature of the theory makes the comparison of the various functions existing in the literature possible and transparent. We have developed the SQM in extended phase space and shown how this method can be generalized to deal with systems subjected to first class constraints. We have proved that Lagrange's method of undermined multipliers yields the quantization of constrained systems in SQM and, in a natural way, results in the Faddeev-Popov conventional path-integral measure for gauge systems. One of the most remarkable features of SQM is that one may quantize even dynamical systems with non-holonomic constraints as it is seen in the case of the stochastic gauge fixing.

- We construct $(\mathrm{N}=2)$-realization of the extended phase space SUSY algebra, discuss the vacuum energy and the topology of super-potentials. The question of spontaneously breaking of extended SUSY deserves further investigation. Therefore, in subsequent paper we will analyze in detail the non-perturbative mechanism for extended phase space SUSY breaking in the instanton picture, and we will show that this indeed has resulted from tunnelling between the classical vacua of the theory. We demonstrate the merits of shape-invariance of exactly solvable extended SUSY potentials, which has underlying algebraic structure, by obtaining analytic expressions for the entire energy spectrum of extended Hamiltonian with Scarf potential without ever referring to underlying differential equation. However, a shape-invariance is not the most general integrability condition as not all exactly solvable potentials seem to be shape-invariant.

- We addressed the classical analog of $(\mathrm{N}=2)$-realization of the supersymmetry algebra. We obtain the integrals of motion. We use the iterative scheme to find the approximate groundstate solutions to the extended Schrödinger-like equation and calculate the parameters which measure the breaking of extended SUSY such as the groundstate energy. The approximation, which went into the derivation of solutions meets our interest that the groundstate energy $\varepsilon$ is supposedly small. This gives direct evidence for the SUSY breaking. However, we calculate a more practical measure for the SUSY breaking, 
in particular in field theories which is the expectation value of an auxiliary field. We analyze in detail the non-perturbative mechanism for extended phase space SUSY breaking in the instanton picture and show that this has resulted from tunneling between the classical vacua of the theory. Finally, we present an analysis on the independent group theoretical methods with nonlinear extensions of lie algebras from the extended phase space SUSY quantum mechanics. Using the factorization procedure we explore the algebraic property of shape invariance and spectrum generating algebra. Most of these Hamiltonians posses this feature and hence are solvable by an independent group theoretical method. We construct the unitary representations of the deformed Lie algebra.

- The OM formalism is the mathematical framework for our physical outlook embodied in the idea that the geometry and fields, with the internal symmetries and all interactions, as well the four major principles of Relativity (Special and General), Quantum, Gauge and Colour Confinement, are derivative. They come into being simultaneously in the stable system of the underlying 'primordial structures' involved in the 'linkage' establishing processes. The OM formalism is the generalization of secondary quantization of the field theory with appropriate expansion over the geometric objects leading to the quantization of geometry drastically different from all existing schemes. We generalize the OM formalism via the concept of the OMM yielding the MW-geometry involving the spacetime continuum and the internal worlds of the given number. In an enlarged framework of the OMM we define and clarify the conceptual basis of subquarks and their characteristics stemming from the various symmetries of the internal worlds. They imply subcolour confinement and gauge principle. By this we have arrived at an entirely satisfactory answer to the question of the physical origin of the Geometry and Fields, the Internal symmetries and interactions, as well the fundamental principles of Relativity, Quantum, Gauge and Subcolour Confinement.

- The value of the hypothesis of existence of the MW-structures defined on the MW-geometry resides in solving of some key problems of the SM, wherein we attempt to suggest a microscopic approach to the properties of particles and interactions. Particularly, we derive the Gell-Mann-Nishijima relation and flavour group, infer the only possible low energy SM particle spectrum, and conclude that the leptons are particles with integer electric and leptonic charges, and free of confinement, while quarks carry fractional electric and baryonic charges, and imply the confinement. We suggest the microscopic theory of the unified electroweak interactions with a small number of free parameters, wherein we exploit the background of the local expanded symmetry $S U(2) \otimes U(1)$ and P-violation. The Weinberg mixing angle is shown to have fixed value at $30^{\circ}$.

Due to the Bose-condensation of relativistic fermion pairs the Higgs bosons have arisen on an analogy of the Cooper pairs in superconductivity. It involves the Higgs bosons as the collective excitations of bound quasi-particle iso-pairs. In the framework of local gauge invariance of the theory incorporated with the P-violation in weak interactions we propose a mechanism providing the Bosecondensation of iso-pairs, which is due to effective attraction between the relativistic fermions caused by the exchange of the mediating induced gauge quanta in the $\mathrm{W}$-world. We consider the fourcomponent Bose-condensate, where due to self-interaction its spin part is vanished. Based on it we show that the field of symmetry-breaking Higgs boson always must be counted off from the gap symmetry restoring value as the point of origin. Then the Higgs boson describes the excitations in the neighbourhood of stable vacuum of the W-world. In contrast to the SM, the suggested approach predicts the electroweak symmetry breakdown in the $W$-world by the VEV of spin zero Higgs bosons and the transmission of electroweak symmetry breaking from the $W$-world to the $M_{4}$ spacetime continuum. The resulting Lagrangian of unified electroweak interactions of leptons and quarks ensues, which in lowest order approximation leads to the Lagrangian of phenomenological SM. In general, the self-energy operator underlies the Yukawa coupling constant, which takes into account a massspectrum of all expected collective excitations of bound quasi-particle pairs.

The implication of quarks into this scheme is carried out in the same manner except that of appearance of quark mixing with Cabibbo angles and the existence of CP-violating complex phase in unitary matrix of quark mixing. The Q-components of the quarks $u^{\prime}, c^{\prime}$ and $t^{\prime}$ contain at least one identical subquark, due to which the partial formfactors gain nonzero values. This underlies the quark mixing with Cabibbo angles. In the case of the leptons these formfactors are vanished and the mixing is absent. The CP-violation stems from the spanning incorporated with the expanded group of global 
rotations. With a simple viewpoint on Higgs sector the masses of leptons and quarks are given.

- We derive the MW-SUSY, which has an algebraic origin in the sense that it has arisen from the subquark algebra defined on the internal worlds, while the nilpotent supercharge operators are derived. Our purpose at first is much easier to handle, by restoring in the first the 'exact' ' MWSUSY. It can be achieved by lifting up each sparticle to corresponding particle state. This enables the sparticle to be included in the same supermultiplet with corresponding particle. Due to different features of particles and sparticles when passing back to physically realistic limit one must have always to distinguish them by introducing an additional discrete internal symmetry, i.e., the multiplicative $Z_{2} \quad R$-parity. The MW-SUSY has realized only on the internal worlds, but not on the spacetime continuum, which are all the ingredients of the broken super-multimanifold (\$MM). Defined on the \$MM it implies the super-algebra different from the conventional SUSY algebra. We write then the most generic renormalizable MW-SUSY action involving gauge and supersymmetric matter frame fields, and, thus, the corresponding generating functional. Therein, we are led to the principal point of drastic change of the standard SUSY scheme to specialize the superpotential to be in such a form, which enables within this framework, further, to build up the MTSM.

- We develop the microscopic approach to the isospinor Higgs boson with self-interaction and Yukawa couplings, wherein the two complex self-interacting isospinor-scalar Higgs doublets $\left(H_{u}, H_{d}\right)$ as well as their spin- $\frac{1}{2}$ SUSY partners $\left(\widetilde{H}_{u}, \widetilde{H}_{d}\right)$ Higgsinos have arisen on the $W$-world as the Bosecondensate. The Higgs mechanism does work in the following way: Before the symmetry was broken in the $W$-world, the 2 complex $S U(2)_{L}$ Higgs doublets had 8 degrees of freedom. Three of them were the would-be Nambu-Goldstone bosons $G^{0}, G^{ \pm}$, which were absorbed to give rise the longitudinal modes of the massive $W$-components of the $Z^{0}$ and $W^{ \pm}$vector bosons, which simultaneously give rise the corresponding $x$ - components too, leaving 5 physical degrees of freedom. The latter consists of a charged Higgs boson pairs $H^{ \pm}$, a CP-odd neutral Higgs boson $A^{0}$, and CP-even neutral Higgs bosons $h^{0}$ and $H^{0}$. The mass eigenstates and would-be Nambu-Goldstone bosons are made of the original gauge-eigenstate fields, where the physical pseudoscalar Higgs boson $A^{0}$ is made of from the imaginary parts of $h_{u}^{0}$ and $h_{d}^{0}$, and is orthogonal to $G^{0}$; while the neutral scalar Higgs bosons are mixtures of the real parts of $h_{u}^{0}$ and $h_{d}^{0}$. The mass of any physical Higgs boson that is SM-like is strictly limited, as are the radiative corrections to the quartic potential terms. We calculated the treelevel masses for these Higgs states (sec.16) and shown that the $h^{0}$ Higgs boson arisen in the internal $W$-world is much heavier of that $Z^{0}$ boson. Such a breaking of the MW-SUSY can be implemented by subtracting back all the explicit soft mass terms formerly introduced for the sparticles. These terms do not reintroduced the quadratic diagrams which motivated the introduction of SUSY framework. Therewith, the boson-fermion cancellation in the above-mentioned problems can be regarded as a consequence of a constraint stemming from holomorphy of the observables, therefore it will be held at the limit too. Thus, we extract the pertinent piece containing only the $\eta$-field components and then in afterwards pass to $M_{4}$ to get the final VMSM yielding the realistic particle spectrum.

- The implication of quarks into the VMSM is carried out in the same way of leptons except that of appearance of quark mixing with Cabibbo angle and the existence of CP-violating complex phase in unitary matrix of quark mixing. The Q-components of the quarks contain at least one identical subquark, due to which the partial formfactors gain nonzero values. This underlies the quark mixing with Cabibbo angles. In lepton's case these formfactors are vanished and lepton mixing is absent. The CP-violation stems from the spanning. Adopting a simple viewpoint on Higgs sector the masses of leptons and quarks are given.

- Employing some features of the TSSD theory, we probe the origin and nature of the phenomenon of inertia effects. We construct the relativistic theory of inertia, which treats the inertia as a distortion of local internal properties of hypothetical 2D master-space. The MS is an indispensable companion of individual particle, without relation to the other matter, embedded in the background 4D-spacetime. The RTI allows to compute the inertial force, acting on an arbitrary point-like observer or particle due to its absolute acceleration. In this framework we essentially improve standard metric and other relevant geometrical structures referred to a noninertial frame for an arbitrary velocities and characteristic acceleration lengths. Despite the totally different and independent physical sources of gravitation and inertia, this approach furnishes justification for the introduction of the WPE. We relate the inertia ef- 
fects to the more general post-Riemannian geometry. We derive a general expression of the relativistic inertial force exerted on the extended spinning body moving in the Rieman-Cartan space.

- We present a standard Lorentz code of motion in a new perspective of supersymmetry. In this, we explore the intermediate, so-called, motion state for a particle moving through the two infinitesimally closed points of original space. The Schwinger transformation function for these points is understood as the successive processes of annihilation of a particle at initial point and time, i.e. the transition from the initial state to the intermediate motion state, and the creation of a particle at final point and time, i.e. the subsequent transition from the intermediate motion state to the final state. The latter is defined on the master space, MS ( $\equiv \underline{M}_{2}$ ), which is prescribed to each particle, without relation to every other particle. Exploring the rigid double transformations of MS-SUSY, we derive SLC as the individual code of a particle in terms of spinors referred to MS. This allows to introduce the physical finite time interval between two events, as integer number of the duration time of atomic double transition of a particle from $M_{4}$ to $\underline{M}_{2}$ and back. The theories with extended $N_{\max }=4$ supersymmetries, as renormalizable flat-space field theories, if only such symmetries are fundamental to nature, lead to the model of ELC in case of the apparent violations of SLC, the possible manifestations of which arise in a similar way in all particle sectors. We show that in the ELC-framework the propagation of the superluminal particle could be consistent with causality, and give a justification of forbiddance of Vavilov-Cherenkov radiation/or analog processes in vacuum. In the framework of local MS-SUSY, we address the inertial effects. The local MS-SUSY can only be implemented if $\underline{M}_{2}$ and $\widetilde{M}_{4}$ are curved (deformed). Whereas the space $\widetilde{M}_{4}$, in order to become on the same footing with the distorted space $\underline{\underline{M}}_{2}$, refers to the accelerated reference frame of a particle, without relation to other matter fields. So, unlike gravitation, a curvature of space-time arises entirely due to the inertial properties of the Lorentz-rotated frame of interest, i.e. a fictitious gravitation which can be globally removed by appropriate coordinate transformations. The only source of graviton and gravitino, therefore, is the acceleration of a particle, because the MS-SUSY is so constructed as to make these two particles just as being the two bosonic and fermionic states of a particle of interest in the background spaces $M_{4}$ and $\underline{M}_{2}$, respectively, or vice versa. Therefore, a coupling of supergravity with matter superfields evidently is absent in resulting theory. Instead, we argue that a deformation/(distortion of local internal properties) of MS is the origin of inertia effects that can be observed by us.

- We briefly outline the issues on the interaction of electrons with the intense radiation: Einstein's transition coefficients for Compton scattering, and the annihilation and creation of electron-positron pairs at intense radiation. Einstein's ideas are developed for free-virtual, virtual-free and free-free transitions for electron-photon scattering at arbitrary intense radiation by splitting Compton scattering into two components. Whereas, we consider the general problem of interaction of electrons with the intense radiation via $s$-photon Compton scattering $s \gamma+e \rightarrow \gamma^{\prime}+e^{\prime}$. In doing this, we introduce a new concept of 'effective photon', and then instead of $s$-photon scattering by electron with an 'effective' four-momentum, with equal footing,, we should consider the scattering of one 'effective photon' by free electron. The Compton scattering is the $s$-channel of the photon-electron interaction. This formalism can be easily extended to the $t$-channel of the photon-electron interaction, namely to processes of annihilation and creation of electron-positron pairs. On the basis of the method of 'effective photons', the integral kinetic equation is derived that describes the time variation of the distribution function of quanta of non-equilibrium intense radiation for their multiphoton Compton scattering on Maxwellian nonrelativistic electrons. The equations of heating and cooling of electron gas are derived. We have continued the study of the process of relaxation of intense radiation on Maxwellian electrons in the general case of any spectral widths and any angular aperture of the radiation beam.

- Finally, we study the much-discussed in literature question of interpretation of the spectral shift of radiation from a distant object in a curved spacetime. We aim to provide a unique definition for the kinetic relative velocity between a source and the observer as measured along the observer's lineof-sight. Extending those geometrical ideas of well-known kinematic spectral shift rule to infinitesimal domain, we try to catch this effect by building a series of infinitesimally displaced shifts and then sum over them in order to find the proper answer to the problem that we wish to address. Thereby, the general equation is the result of a series of infinitesimal stretching of the proper space scale factor in Riemannian space-time, whereas the path of a luminous source appears nowhere, thus this equation 
does not relate to the special choice of transport path. A resulting general relationship between the spectral shift and the kinetic relative velocity is utterly distinct from a familiar global Doppler shift. We discuss the implications for a particular case when adjacent observers are being in free fall and populated along the null geodesic, so that the kinetic relative velocity of luminous source is reduced to global Doppler velocity as studied by Synge. Moreover, the implications for the spatially homogeneous and isotropic Robertson-Walker space-time of standard cosmological model leads to cosmological consequences that resulting kinetic recession velocity of a distant astronomical object is always subluminal even for large redshifts of order one or more and, thus, it does not violate the fundamental physical principle of causality. That is, in the framework of 'stretching of space' point of view of the spatially homogeneous and isotropic RW space-time of standard cosmological model, we overcome an ambiguity of the procedure of parallel transport of four-velocity of source along the null geodesic to an observer by an alternative study of a 'lookforward' history of expanding universe. We use a way of separating the cosmological redshifts into infinitesimally displaced 'relative' redshift bins and sum over them to achieve an unique definition of the kinetic recession velocity of comoving astronomical object. A stemming relationship of overall cosmological redshift and kinetic recession velocity is utterly distinct from a familiar global Doppler shift formula. Nevertheless, in particular case of along the null geodesic, a general solution is reduced to a global Doppler shift.

\section{References}

Adler S. L., 2021, Int.J.Mod.Phys., A14, issue 12, 1911

Einstein A., Ehrenfest P., 1923, Z. Phys., Bd. 19, S. 301-306

Mihalas D., 1978, Stellar atmospheres, San Francisco: Freeman, W H

Nambu Y., Jona-Lasinio G., 1961, Dynamical model of elementary particles based on an analogy with superconductivity, Phys. Rev., 122,345

Pauli W., 1923, Z. Phys., Bd. 18, S. 272-277

Synge J. L., 1960, Relativity: The General Theory, North-Hollaand, Amsterdam

Ter-Kazarian G., 1884, Operator Manifold Approach to Geometry and Particle Physics, I.C.T.P., Trieste, IC/94/290, No1, pp.1-76, Italy; [arXiv:hep,th/9510110]

Ter-Kazarian G., 1984a, Corrections to Inverse Compton Scattering, Astrofizika, 21, Issue 2, pp. , 345-352

Ter-Kazarian G., 1984b, The Energy Exchange Processes Between Electrons and Photons At the Intense Radiation, part I, Astrofizika, 21, pp. 609-625

Ter-Kazarian G., 1984c, The Einstein's Radiation Coefficients for the Compton Scattering, Akad. Nauk SSSR, Doklady, 276, No1, pp.598-603. In Russian

Ter-Kazarian G., 1984d, Electron's Motion Corrections to Inverse Compton Scattering, Akad. Nauk SSSR, Doklady (ISSN 00023264), 276, no. 1, 1984, p. 106-110. In Russian

Ter-Kazarian G., 1984e, The Einstein's Coefficients for the Compton Scattering, Communications of the Byurakan Astrophysical Observatory (ComBAO), 55, pp.65-69

Ter-Kazarian G., 1986, Distortion of the Space-Time, Textbook:"Selected Questions of Theoretical and Mathematical Physics". Vol. N5322-B86, No1, 62-146, VINITI, Moscow, Russia

Ter-Kazarian G., 1987, The Energy Exchange Processes Between Electrons and Photons At the Intense Radiation, part II., Astrofizika, 27, pp. 509-522

Ter-Kazarian G., 1989a, Supermassive Equilibrium Configurations of Superdense Matter, Akademiia Nauk SSSR, Doklady (ISSN 0002-3264), In Russian, 309, No.1, 97-101

Ter-Kazarian G., 1989b, On Study of Equilibrium Configuration of Protomatter, Part I, Astrofizika, 31, Issue 2, 345

Ter-Kazarian G., 1989c, The Theory of Distortion of Space-Time Continuum, ComBAO, 62, 1-123

Ter-Kazarian G., 1990, On Study of Equilibrium Configuration of Protomatter, Part II, Astrofizika, 33, 143-153

Ter-Kazarian G., 1991, On the study of internal structure of superdense celestial bodies. Astrofizika, 35, Issue 2,3, 335-372

Ter-Kazarian G., 1992, An Introduction to the Theory of Internal Structure of Supermassive Compact Celestial Bodies, Astrophysics \& Space Sci., 194, No1, 1-129 
A new look at some aspects of geometry, particle physics, inertia, radiation and cosmology

Ter-Kazarian G., 1996, Operator Manifold Approach to Geometry and Particle Physics: General Gauge Principle \& Gravitation, Astrophys. \& Space Sci., 241, No2, pp.161-209

Ter-Kazarian G., 1997, Gravitation Gauge Group, Nuovo Cimento, 112B, No 6, 825-838

Ter-Kazarian G., 1999a, Operator Manifold Formalism, CPT-99/P.3918, CPT-CNRS, URL:www.cpt.univ-mrs.fr, Marseille, France, No1, pp.1-30; arxiv:9812181[hep,th]

Ter-Kazarian G., 1999b, Microscopic Theory of The Standard Model, CPT- 99/P.3919, CPT- CNRS, URL:www.cpt.univ-mrs.fr , Marseille, France, No1, pp.1-42; arxiv:9812182[hep-th]; arXiv:9710089[hep,th]

Ter-Kazarian G., 2001a, Protomatter and EHE C.R., Japan Phys. Soc. Jpn., 70, S. B., 84-98

Ter-Kazarian G., 2001b, Microscopic Theory of the Standard Model, CPT-2001/P.4269, CPT-CNRS, pp.1-35, URL:www.cpt.univmrs.fr, Marseille, France, arXiv:0007077[hep,ph]

Ter-Kazarian G., 2001c, Supersymmetric Microscopic Theory of the Standard Model, CPT-2001/P.4270, CPT-CNRS, pp.1-34, URL:www.cpt.univ-mrs.fr, Marseille, France, arXiv:0009046[hep,ph]

Ter-Kazarian G., 2009, An Extended Phase Space SUSY Quantum Mechanics, J. Phys. A: Math. Theor., 42, 055302

Ter-Kazarian G., 2010, Gravitation and inertia; a rearrangement of vacuum in gravity; Astrophysics \& Space Sci., 327, 91-109, arxiv:1001.2990[gr,qc]

Ter-Kazarian G., 2011, Two-step spacetime deformation-induced dynamical torsion, Class. Quantum Grav., 28, 055003 (19pp); stacks.iop.org/CQG/28/000000; arxiv:1102.24911[gr,qc]

Ter-Kazarian G., 2012, Spacetime deformation-induced inertia effects, Advances in Mathematical Physics, Article ID 692030, 1-41, http://dx.doi.org/10.1155/2012/692030

Ter-Kazarian G., 2013a, Classical analog of extended phase space SUSY and its breaking, ISRN Mathematical Physics, 2013, Article ID 639424; http://dx.doi.org/10.1155/2013/639424

Ter-Kazarian G., 2013b, Is there an inertia due to the supersymmetry, 2013, arxiv:1303.3180[gr,qc]

Ter-Kazarian G., 2014, Ultra-high energy neutrino fluxes from supermassive AGN black holes, Astrophys. \& Space Sci., 349, 919-938

Ter-Kazarian G., 2015, Modified Theories of Gravitation Behind the Spacetime Deformation, Physics Research International, 2015, Article ID 152846, 1-19,

http://dx.doi.org/10.1155/2015/152846

Ter-Kazarian G., 2016a, Rotating black holes in microscopic theory: the implications for periodic source M82X-2, Advances in Astrophysics, 1, issue 3, 201-223

Ter-Kazarian G., 2016b, On the Physical Nature of the Source of Ultraluminous X-ray Pulsations, Astrophys. \& Space Sci., 361, issue 1, 20pp, DOI 10.1007/s10509

Ter-Kazarian G., 2021a, On the kinematic interpretation of cosmological redshifts, ComBAO, 68, issue 1, pp. 12-31, https://doi.org/10.52526/25792776

Ter-Kazarian G., 2021b, Unique definition of relative speed along the line of sight of a luminous object in a Riemannian space-time, ComBAO, 68, issue 1, pp. 38-49, https://doi.org/10.52526/25792776

Ter-Kazarian G., 2021c, Ambartsumian's cosmogony and the key mysteries of high energy astrophysics, ComBAO, to appear

Ter-Kazarian G., Sargsyan L., 2013, Signature of plausible accreting supermassive black holes in Mrk 261/262 and Mrk266, Advances in Astronomy, 2013, Article ID 710906, http://dx.doi.org/10.1155/2013/710906

Ter-Kazarian G., Shidhani S., 2017, Plausible Intermediate Mass Black Hole from M82X-2 X-Ray Pulsations, Advances in Astrophysics, 2, issue 3, 162-183

Ter-Kazarian G., Shidhani S., 2019, A study of 137 intermediate mass black hole candidates, Astrophys. Space Sci., 364, Issue 10, article id. $165,23 \mathrm{pp}$

Ter-Kazarian G., Sobouti Y., 2008, An Extended Phase Space Stochastic Quantization Of Constrained Hamiltonian Systems, J. Phys. A: Math. Theor., 41, 315303

Ter-Kazarian G., Yerknapetian K., 1995, On the Internal Structure of Supermassive Compact Celestial Bodies, Astrofizika, 38, No4, 659-666

Ter-Kazarian G., Khachatryan V., Yerknapetyan K., 2003, Neutrino Cooling and Fueling at Disk Accretion Onto the SPC in AGN, and Origin of EHE G.R.s, "The Universe Viewed in Gamma-rays", ICRR, University of Tokyo. Universal Academy Press, Inc., BR-Hongo-5 Bldg., 6-16-2 Hongo Bunkyo-ku, Tokyo, Japan

Ter-Kazarian G., Terzyan Y., Khachikian E. e. a., 2006, On Multi-Nuclei Features of Some Markarian Galaxies, Monthly Notice of Royal Astron. Soc., 368, 461-470

Ter-Kazarian G., Shidhani S., Sargsyan L., 2007, Neutrino Radiation of the AGN Black Holes, Astrophys. \& Space Sci., 310, issue $2,93-110$ 